\title{
HEATS OF COMBUSTION OF ORGANIC COMPOUNDS
}

\author{
By M. S. Kharasch
}

\section{ABSTRACT}

The literature on the heats of combustion of organic compounds is critically reviewed. A table of "best" values for this constant has been compiled covering all available information on record in the literature.

A method for calculating the heat of combustion from the structural formula of the compound is described, and the values calculated according to this method are tabulated for comparison with the observed values.

The comparison demonstrates that the calculated values are sufficiently accurate for most practical purposes. The difference between calculated and observed values seldom exceeds 1 to 2 per cent except in cases where there is reason to doubt the accuracy of the observed value. In most cases the two values agree within the accuracy of the observed value.

\section{CONTENTS}

I. Introduction

II. Standards for combustion calorimetry

III. Choice of data_. 363

IV. Abbreviations, units, and conventions 363

V. Calculation of heat of combustion 364

VI. Structural correction factors

VII. Index of compounds, by classes

1. $\mathrm{CH}$ compounds_._........ 370

2. $\mathrm{CHO}$ compounds

3. $\mathrm{N}$ compounds $\ldots \ldots$

4. Halogen and sulphur compounds

VIII. Tables of data

IX. Bibliography

X. Index of compounds, by formula_... 426

\section{INTRODUCTION}

In the present collection of the data on the heats of combustion of organic compounds, an attempt has been made to select the best available value for each compound. The work has been particularly difficult because only a few authors give the necessary information concerning the unit of heat and the thermochemical corrections used, and without this information it is impossible to bring the values to a common basis.

The thermochemical data recorded in the tables have been obtained by the use of the following thermochemical methods: (1) The universal-burner method, (2) the bomb method. 
The "universal-burner" method, in the hands of Thomsen, gave results which agreed on the average to not better than 0.5 per cent. Owing to the design of his apparatus, jt is applicable only to gases or to substances easily volatile at room temperature, and his values are to be trusted only to the extent to which this condition was realized. This seems to be the consensus of opinion of most workers in thermochemistry. It is, however, necessary to apply two corrections to his values in order to bring them into accord with modern determinations. Both of these corrections are negative. The first one $(-0.3$ per cent) corrects his thermometer readings to the hydrogen scale, and the second one ( -0.1 per cent) corrects for the new determinations of the heat capacity of water. Only the values so corrected are recorded in the tables.

The "bomb-calorimeter" method was first used by Andrews ${ }^{1}$ in 1848, the determinations being carried out at a pressure of one atmosphere. In 1883 Berthelot reintroduced the method and improved it considerably. The combustions by this improved method were carried out with oxygen under pressure. The details of the technic have since undergone revolutionary revisions. For particulars the reader is referred to the articles of Richards and his collaborators; Roth and collaborators; Dickinson, Verkade, and collaborators; and Swietoslawski and collaborators.

Of the four different procedures that have been proposed for the measurement of heats of combustion by means of a calorimetric bomb, only two have attained considerable use. ${ }^{2}$ These are known as the "ordinary" or "common" method and the "adiabatic" method. In the "common" method the rise of temperature of the calorimeter is observed while the "jacket" temperature is kept constant. In the "adiabatic" method the temperature of the "jacket" is kept the same as the temperature of the calorimeter and only the initial and final temperatures measured. This latter method may be called the "American adiabatic" method, for it was first proposed by S. W. Holman ${ }^{3}$ and refined thoroughly by Richards and his collaborators. It has been used extensively only in the United States.

It should be stated at this point that the results of the two methods agree within the limits of experimental error. ${ }^{4}$

1 Andrews, Pogg. Ann., 75, p. 27; 1848.

2 The other two methods are those of Hosehus and Fery.

3 S. W. Holman, Proc. Am. Acad., 31, p. 252; 1895.

' Dickinson, Bull. Bureau of Standards, 11, p. 243; 1915; Schläpfer and Fioroni, Helv. chim. Acta, 6. p. $725 ; 1923$. 
The method of calibrating the calorimetric system is of the greatest importance. ${ }^{5}$ The methods which have been at times employed by the investigators and pertain to the values recorded are as follows: (1) Calibration by the electrical method; (2) thermal calibration; (3) the additive calibration.

1. The electrical calibration consists in supplying electrically a measured amount of energy to the calorimeter and measuring the temperature rise. The results of such observations give directly the heat capacity of the calorimeter and its contents in joules per degree. The value in calories is then obtained by dividing by the mechanical equivalent of heat. This method forms the basis of the work of Fischer and Wrede; Wrede, Jaeger, and von Steinwehr; Dickinson, Roth, Swartz, Moureu, and André. The work of these investigators is very thorough and of a high order of accuracy.

2. In the thermal method of calibration a standard substance of known heat of combustion is burned in the calorimeter. Where the other calorimetric measurements have been properly carried out this method, using one of the standard substances mentioned below, yields reliable results.

3. In the additive-calibration method the heat capacity of the calorimeter is obtained by adding together the heat capacities of its parts. This method has been used extensively by some French and some Russian investigators. While the work has been carried out with a great deal of zeal, yet essential details of procedure are often entirely lacking and it is thus usually impossible to compare the values with those of other workers. These statements apply to work of Berthelot and his collaborators, Louguinine, Zubov, and a number of other investigators. Recently the values of Zubov's work have been put upon a better basis by Swietoslawski, to whom Zubov turned over most of his experimental material. From the method

${ }_{5}$ The following are some of the most important references dealing with the calibration of the calorimetric system:

1. Bestimmung des Wasserwertes eines Berthelot'schen Kalorimeter in elektrischen Einheiten, W. Jaeger und H. von Steinwehr, Verhandl. Deutsch. Phys. Ges., 5, p. 50; 1903; and 5, p. 353; 1903.

2. Concerning the adiabatic determination of the heat of combusion of organic substances, Richards, Henderson and Frevert, Proc. Am. Acad., 42, p. 573; 1907.

3. Beitrag zur kalorimetrischen Messung von Verbrennungswärme, W. Jaeger and H. von Steinwehr, Zs. f. Phys. Chem., 53, p. 153; 1905.

4. Eichung eines Berthelot'schen Verbrennungskalorimeters in elektrischen Einheiten mittels des Platinthermometers, W. Jaeger and H. von Steinwehr, Ann. d. Phys. (4), 21, p. 23; 1906.

5. Ueber die Korrektur für die Wärmestrahlung bei Kalorimetrischen Versuchen, A. Schukarew, Zs. f.

Phys. Chem., 56, p. 453; 1906.

6. An accurate Calorimeter, White, Phys. Rev., 25, p. 137; 1907.

7. Eichung des Verbrennungskalorimeter und Arbeitsweise, Roth, Lieb. Ann., 373, p. 249; 1910.

8. Lag effect and other errors of calorimetry, White, Phys. Rev., 31, p. 562; 1910.

9. A calorimetric arrangement for the new bomb, Phillippe Landrieu, Bull. Soc. Chim.,.37, p. 1340; 1925. 
employed by Swietoslawski in correcting Zubov's data, it is evident that Zubov applied most thermometric corrections, except that the heat capacity of his calorimetric system has been erroneously determined. These recalculated values of Zubov are in better agreement with more modern values and are the ones recorded in the tables.

\section{STANDARDS FOR COMBUSTION CALORIMETRY}

Of the three substances which have been used in the past as standards in combustion calorimetry, only one has been shown by recent researches to meet the requirements of a primary standard. At the third conference of the International Union of Pure and Applied Chemistry held at Lyons ${ }^{6}{ }^{6}$ benzoic acid was adopted as the primary standard. The value chosen for its heat of combustion was the one found by Dickinson, ${ }^{7}$ namely, $6,324, \mathrm{~g}^{\mathrm{c}} \mathrm{cal}_{15}$ per gram in air or 6,319 g-cal ${ }_{15}$ per gram in vacuo. ${ }^{8}$ It is more or less tacitly assumed that the above value holds for an isothermal heat of combustion in the neighborhood of $20^{\circ} .{ }^{9}$ When converted into absolute joules these values become 26,466 and 26,445, respectively. Dickinson's value was obtained by absolute electrical standardization of his calorimetric system, and the measurements were carried out by the ordinary as well as the adiabatic method.

It is of importance to note here the determinations which have been made of the ratios of the heats of combustion of benzoic acid, naphthalene, and cane sugar. For these determinations Richards and his collaborators employed only the adiabatic method, Verkade and his collaborators the ordinary method, while Swietoslawski and his collaborators and Schläpfer and Fioroni employed both the adiabatic and ordinary methods. These ratios, as summed up by Schläpfer and Fioroni as a result of a very thorough investigation both by the ordinary and the adiabatic methods, are:

$$
\frac{\text { Naphthalene }}{\text { Benzoic acid }}=\underset{\text { (air) }}{1.5201} \frac{\text { Benzoic acid }}{\text { Sucrose }}=\underset{\text { (air) }}{1.6028} \frac{\text { Naphthalene }}{\text { Sucrose }}=\frac{2.4364}{\text { (air) }}
$$

These ratios are quite similar to those obtained by Dickinson and by Verkade and Coops, jr. ${ }^{10}$

Secondary Standards.--Recently Verkade and Coops, $\mathrm{jr} .{ }^{11}$ have suggested salicylic acid as a secondary standard. Their suggestion, backed by a large amount of information, certainly merits considera-

- Germany was not represented at that conference.

7 Bull. Bur. of Standards, 11, p. 243; 1915.

${ }^{8}$ See W. Swietoslawski's defense of this value, J. Chim. Phys., 2\%, p. 583; 1925; and P. E. Verkade and J. Coops, Z. Physik. Chem., 118, p. 123; 1925.

${ }^{9}$ See Rec. Trav. Chim., 4, p. $800 ; 1925$, for the temperature coefficient of the heats of combustion of benzoic and salicylic acids.

10 P. E. Verkade and Coops, jr., Rec. Trav. Chim., 42, p. 223; 1923.

11 P. E. Verkade and Coops, jr., Rec. Tr. Chim., 43, p. 561; 1924 . Note also the discussion by Swietosi lawski upon the establishment of such a secondary standard, Bull. Soc. Chim. (4), 3\%, p. 84; 1925. 
tion. The value for salicylic acid recommended by these investigators is $5,242 \mathrm{~g}_{\text {-cal }}{ }_{15}$ per gram in air and $5,238 \mathrm{~g}$-cal ${ }_{15}$ per gram in vacuo, that is, $5,242 \times 4.185=22,699$ and $5,238 \times 4.185=21,921$ absolute joules, respectively.

\section{CHOICE OF DATA}

Since in the tables below only one value is as a rule given for each compound, it has been necessary to exercise a certain amount of arbitrariness in the choice, but whenever possible the opinions of all workers in thermochemistry, as expressed in their articles, have been taken into account. Naturally, where an author has described carefully his method of procedure, corrections used, etc., or where his method has been sufficiently well established, his values were given preference over those of an author who merely recorded the heats of combustion obtained. The names of all the investigators who have determined the heats of combustion of each compound are, however, recorded. When only one value for a compound is quoted, irrespective of whether or not the value is very reliable, it has been thought desirable to make available even this approximate result. On the whole, for the guidance of the users of the tables, it may be stated that the work of many of the French investigators is not in complete agreement with the best modern determinations. The values are on the whole rather high, but no factor can be employed to correct them, for the variations from author to author are too large. However, the maximum error in most cases is not larger than from 1 to 1.5 per cent.

Preference has been given also to Thomsen's values for gases and for easily volatile compounds over those of Berthelot and his collaborators. The order, however, was reversed for difficultly volatile substances, for Thomsen's values for such compounds are too high.

\section{ABBREVIATIONS, UNITS, AND CONVENTIONS}

UNIT EMPLOYED.-The heats of combustion recorded are expressed in absolute kilojoules (at constant pressure) per gram molecular weight of substance in vacuo. Where the investigators indicated the unit of heat employed by them that unit of heat was multiplied by the proper factor to convert the value in to absolute kilojoules, otherwise the $18^{\circ}$ calorie was assumed to have been used..$^{12}$

VACUUM CoRRECTION. - The vacuum correction in very few cases amounts to more than 0.13 to 0.16 per cent. It is quite evident, therefore, that where the accuracy of the method, experimental procedure, and corrections used apparently introduced a much larger error, such a correction is of very little consequence as far as the

12 The factors used to convert into joules were the ones adopted by the International Critical Tables. 
accuracy of the absolute value is concerned. However, it was felt desirable to bring the values to a common basis, and for that reason the values of the investigators have been corrected ad vacuum whenever there seemed the slightest justification for it and when the specific gravity of the substances was known. Of the investigators who have done considerable work in thermochemistry and whose results were sufficiently accurate to merit this correction, we might mention Stohmann and collaborators; Zubov, ${ }^{13}$ Roth, Fischer and Wrede; Wrede, Dickinson, Richards and collaborators; Swietoslawski and collaborators; Verkade and collaborators, and a few others. Of course, some of these investigators have themselves applied the correction and record their result upon the basis of weight in vacuo.

Physical State.-Unless otherwise specified (by $g=$ gas, $v=$ vapor, $s=$ solid), the values recorded refer to the combustion of the substance in the liquid state, the final products of combustion being gaseous carbon dioxide, liquid water, and nitrogen gas, for $\mathrm{C}, \mathrm{H}, \mathrm{N}$ compounds. In the case of compounds containing other elements consult the discussion under the individual headings.

\section{CALCULATION OF HEAT OF COMBUSTION}

The calculated values recorded in the tables refer to the heat of combustion of the substance in the liquid state. Whenever, therefore, the heat of combustion of a solid substance is recorded, it is necessary, for the purpose of comparison, to convert that value to the liquid basis. In making that conversion it is necessary, for precision work, to know the molecular heat of fusion referred to $18^{\circ} \mathrm{C}$. However, no error of any magnitude is introduced into the calculation of the heat of combustion of a liquid substance by merely subtracting the value of the molecular heat of fusion at the melting point from the heat of combustion in the solid state.

The general basis of calculating heats of combustion is discussed in the paper of Kharasch and Sher. ${ }^{14}$ Since the publication of this paper a great deal of experimental work has been carried out by the writer and various collaborators, which enables us to elucidate more fully the general formulas employed. This will be done in papers which will appear elsewhere. To conserve space, factors used will be discussed very briefly, and the user of the table is invited to consult the original papers for the theoretical background and postulates.

It is assumed that, whenever an organic substance is burned in oxygen, the heat generated is due to the interdisplacement of the electrons between the carbon and oxygen atoms. It is assumed, also, that the net amount of these energy interchanges in the form of heat

13 The corrected values given by Swietoslawski (loc. cit.).

14 J. Phys. Chem., 29, pp. 625 to 658; 1925. 
is equal to $26.05 \mathrm{~kg}$-cal ${ }_{15}$ per electron per mole, if the initial and final stages correspond to the arrangement the electron occupies around the carbon nucleus in methane and in carbon dioxide, respectively.

It is easy to perceive, therefore, that since the factor 26.05 corresponds only to certain definite initial and final stages of the electron, whenever a substance is burned which contains some electrons displaced from that position the calculated value should be either smaller or larger than the experimental value, depending upon whether the electrons are nearer or farther from the carbon nucleus than those of our reference position; that is, the arrangement of electrons around the carbon nucelus in methane.

In the case of carbon compounds it is assumed that a sharing of electrons may exist, as represented below. The lines merely indicate the distance which the electrons forming the bond may occupy with respect to the two carbon nuclei $\mathrm{A}$ and $\mathrm{B}$.

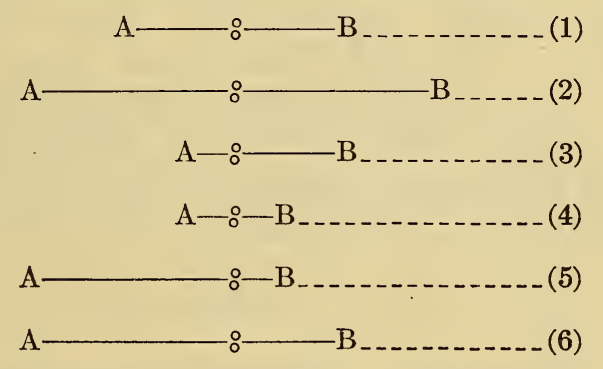

Fig. 1.

Furthermore, if (1) denotes the arrangement of the pair of electrons linking the carbon nuclei in ethane and the expression for the heat of combustion of that compound is $Q=26.05 \times N$, where $N$ denotes the number of electrons, then it is self-evident that if (2), (3), (4), (5), and (6) differ from ethane only in the arrangement of one pair of electrons, then the expression for the heat of combustion of these compounds should be:

(1) $Q=26.05 \times N$

(2) $Q=26.05 \times N+\mathrm{a}$

(3) $Q=26.05 \times N-\mathrm{b}$

(4) $Q=26.05 \times N-\mathrm{c}$, where $\mathrm{c}$ is larger than $\mathrm{b}$.

(5) $Q=26.05 \times N+\mathrm{d}-\mathrm{e}$

(6) $Q=26.05 \times N+\mathrm{f}$

Bonds of the type (5) and (6) need not be considered here, for (6) is merely a special case of (2) and in (5) the two factors ordinarily cancel one another.

The four distinct types of bonds may be illustrated by a consideration of the following molecules: .

1. Bond of type 1.-Aliphatic hydrocarbons, ethane as a representative compound. 
2. Bond of type 2.-A carbon-to-carbon linkage in which both groups are weakly electronegative, such as a $\mathrm{COOH}$ next to $\mathrm{COOH}$ as in oxalic acid or $\mathrm{C}=\mathrm{O}$ next to $\mathrm{COOH}$ as in $\mathrm{CH}_{3} \cdot \mathrm{CO} \cdot \mathrm{COOH}$ or two triphenylmethyl nuclei $\left(\mathrm{C}_{6} \mathrm{H}_{5}\right)_{3} \cdot \mathrm{C}: \mathrm{C}\left(\mathrm{C}_{6} \mathrm{H}_{5}\right)_{3}$.

3. A bond between a carbon of an aliphatic radical or any other $\mathrm{C}$-atom and a carbon atom of a strongly electronegative radical such as phenyl; ${ }^{15}$ thus, $\mathrm{C}_{6} \mathrm{H}_{5}$. $\mathrm{CH}_{3}$.

4. A bond between two carbon atoms of two strongly electronegative radicals, such as $\mathrm{C}_{6} \mathrm{H}_{5} \cdot \mathrm{C}_{6} \mathrm{H}_{5}$, naphthalene, anthracene, etc.

We have thus a general expression for the heat of combustion of organic compounds, $Q=26.05 \times N$ plus certain correction factors for those electrons that are displaced from the reference position, the sign of the correction factor indicating, except in the case of carbonoxygen bonds, whether the electrons are displaced away from the nucleus of the carbon atoms or toward the nucleus.

The correction factors, together with some illustrations, are given below with the proper sign and should always be taken into account whenever calculations are made. In the table only the type formulas will be given. Thus, for example, the heat of combustion of aromatic acids is given by the expression $Q=26.05 \times N-3.5$, but in calculating the heat of combustion of a substance such as 0 -toluic acid, we have $Q=26.05 \times 36-3.5 \times 2=930.8$, the 3.5 being the correction for the bond as in type 3 , and there are two such bonds.

\section{STRUCTURAL CORRECTION FACTORS}

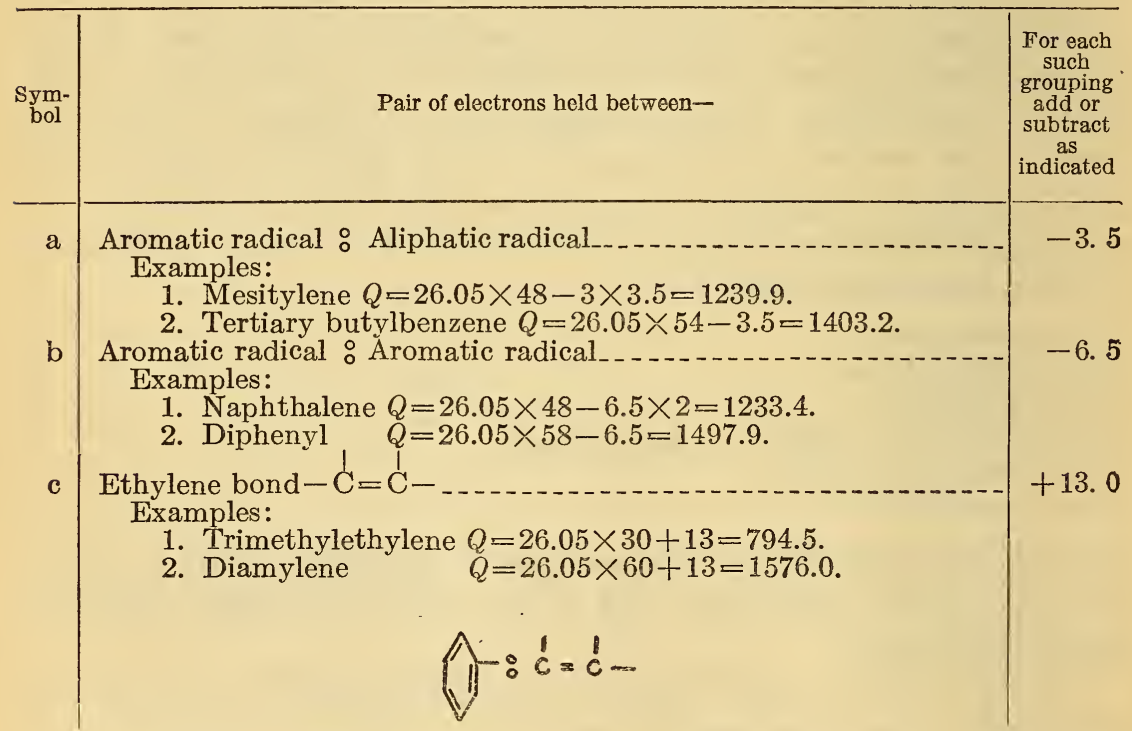

15 Consult paper of Kharasch and Marker, J. A. C. S., 48, p. 3130; 1926 , for a table of reiative electronegativity of organic radicals, and also Kharasch and Flenner (to be published). 


\section{STRUCTURAL CORRECTION FACTORS-Continued}

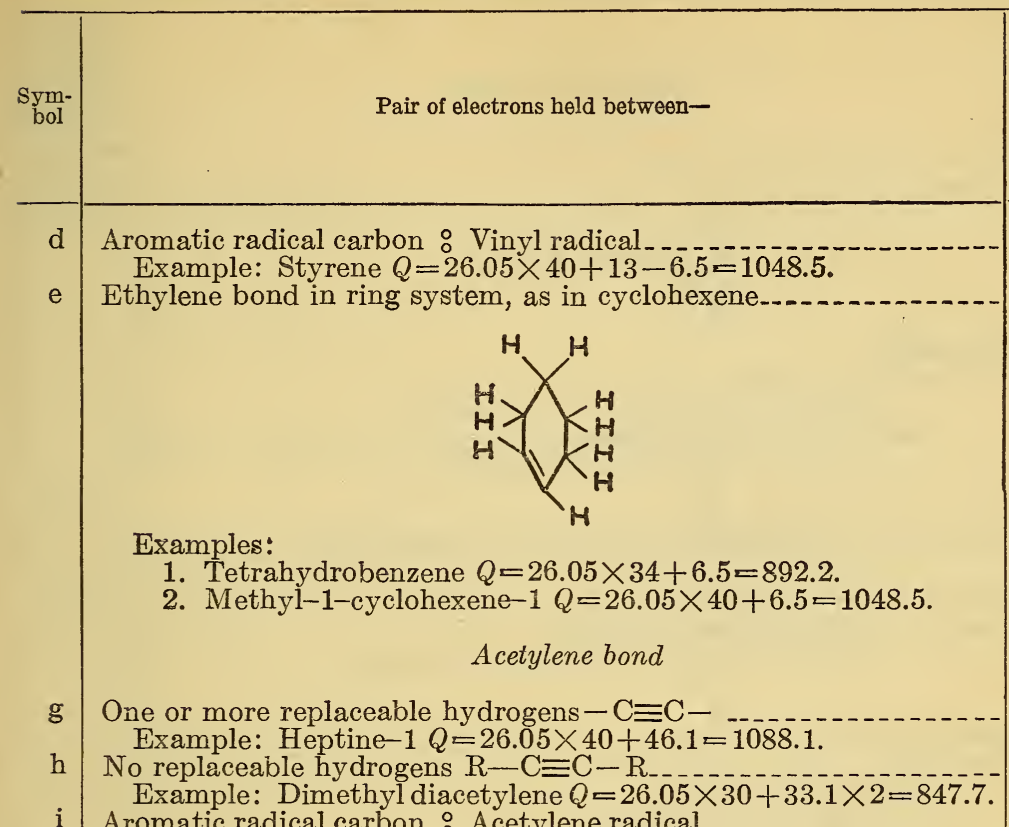

Aromatic radical carbon : Acetylene radical

$$
\widehat{A}: c=c-
$$

Example: Phenyl ethine $Q=26.05 \times 38+46.1-6.5=1029.5$.

\section{Alcohols}

Aliphatic radical : Hydroxyl group (Primary alcohol)

Example: Methyl alcohol $Q=26.05 \times 6+13=169.3$.

Examples:

1. Cyclohexanol $Q=26.05 \times 34+6.5=892.7$.

2. Amyl phenyl propargylalcohol $Q=26.05 \times 72+33.1+6.5$ $-3.5=1911.7$.

Tertiary radical : Hydroxyl group (Tertiary alcohol)

1. Methyl diethyl carbinol $Q=26.05 \times 36+3.5=941.3$.

2. Diphenyl phenyl ethinyl carbinol. $Q=26.05 \times 98+33.1+3.5-6.5-3.5 .2=2576.0$.

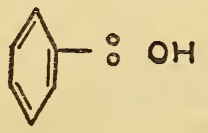

Examples:

1. $m$-Cresol $Q=26.05 \times 34+3.5-3.5=885.7$.

2. Thymohydroquinol $Q=26.05 \times 50+3.5 \times 2-3.5 \times 2=1302.5$.

1. Glycol acetal $Q=26.05 \times 20+19.5 \times 2=560.1$.

2. Dipropyl methylal $Q=26.05 \times 40+19.5 \times 2=1081.1$. 


\section{STRUCTURAL CORRECTION FACTORS-Continued}

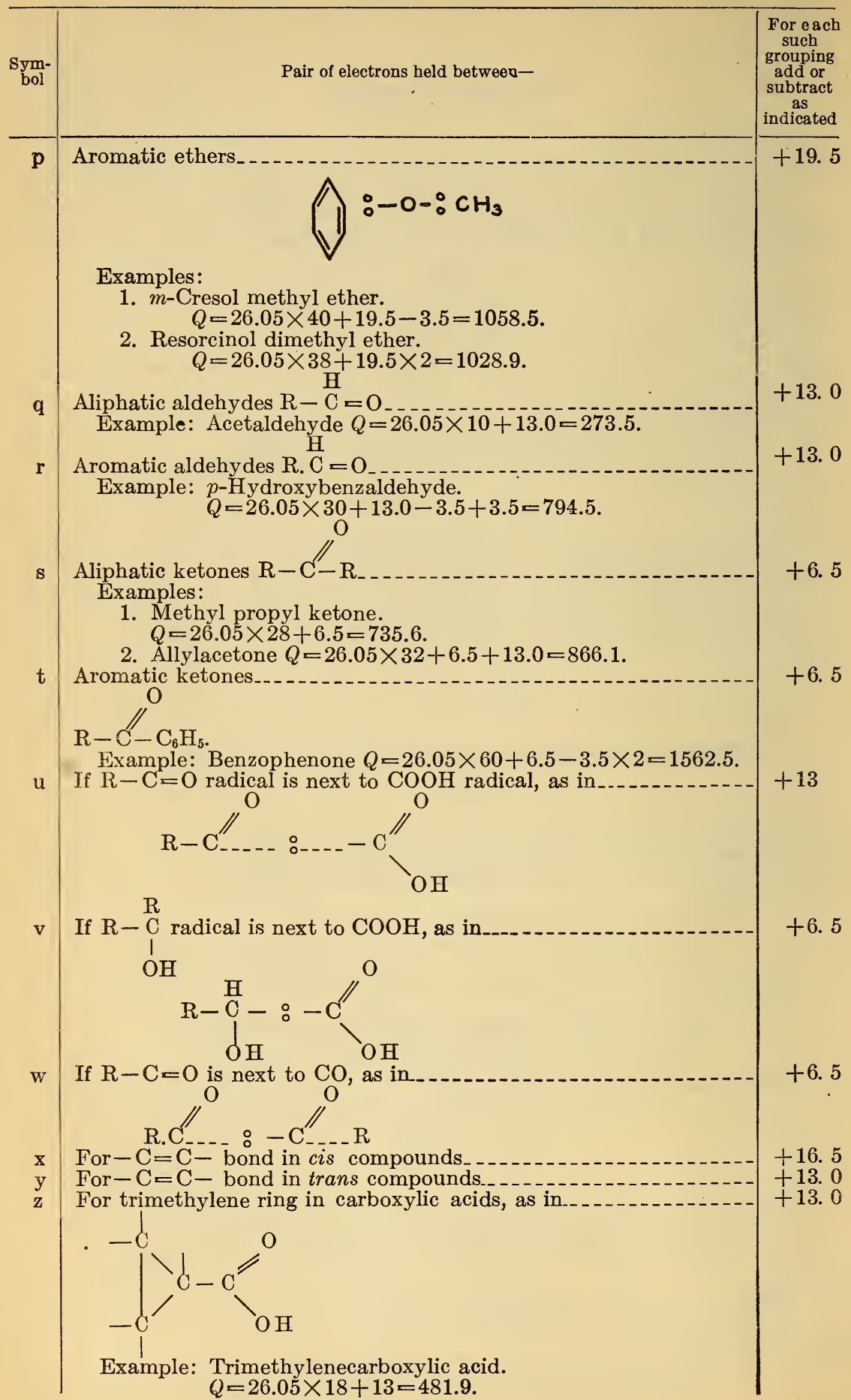




\section{STRUCTURAL CORRECTION FACTORS-Continued}

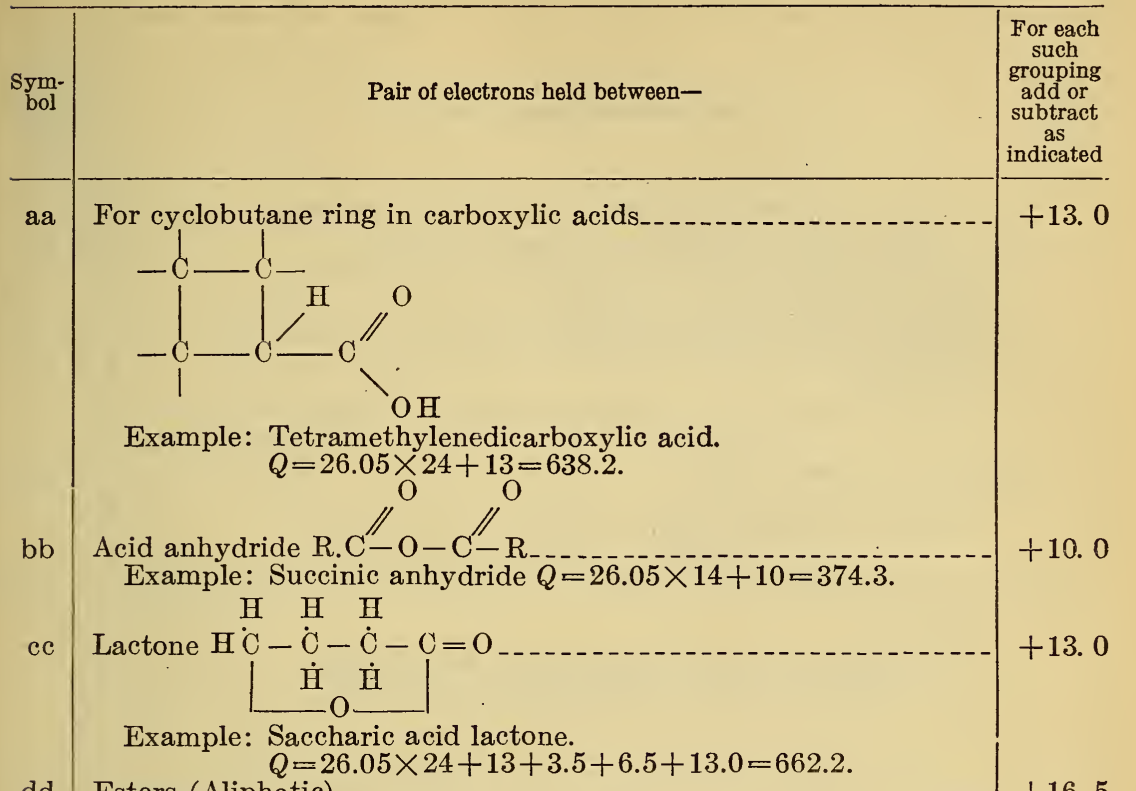

dd

Esters (Aliphatic)

$$
Q=26.05 \times 24+13+3.5+6.5+13.0=662.2 \text {. }
$$<smiles>[R]OC([R])=O</smiles>

Example: Methyl acetate $Q=26.05 \times 14+13=381.2$.

ee

Aliphatic radical : $\mathrm{NH}_{2-}$

(Primary aliphatic amine)

Example: Propyl amine $Q=26.05 \times 21+13=560.1$.

Aliphatic radical : $\mathrm{N}:-$ Aliphatic radical

(Secondary aliphatic amine)

Example:

Benzylethylamine $Q=26.05 \times 49+19.5-3.4=1292.5$.

Aliphatic radical : $\mathrm{N}:$ (Aliphatic radical)

(Tertiary aliphatic amines)

$\mathrm{hh}$

Aromatic radical carbon : $-\mathrm{N}$ (Ammonia type of nitrogen)

Aromatic radical : $-\mathrm{H}-\mathrm{H}$

(Primary aromatic amine)

Example: $p$-Toluidine $Q=26.05 \times 37+6.5-3.5-3.5=963.3$.

Aromatic radical : $\frac{\mathrm{H}}{\mathrm{N}}$ :- aromatic radical

Example:

(Secondary aromatic amine)

Diphenylamine $Q=26.05 \times 59+13.0-3.5 \times 2=1542.9$.

Aromatic radic

Example:

Triphenylamine $Q=26.05 \times 87+19.5-3.5 \times 3=2275.3$.

$\mathrm{mm}$
$+19.5$

$+26.0$

$-3.5$

$+13.0$

$+19.5$ 


\section{STRUCTURAL CORRECTION FACTORS-Continued}

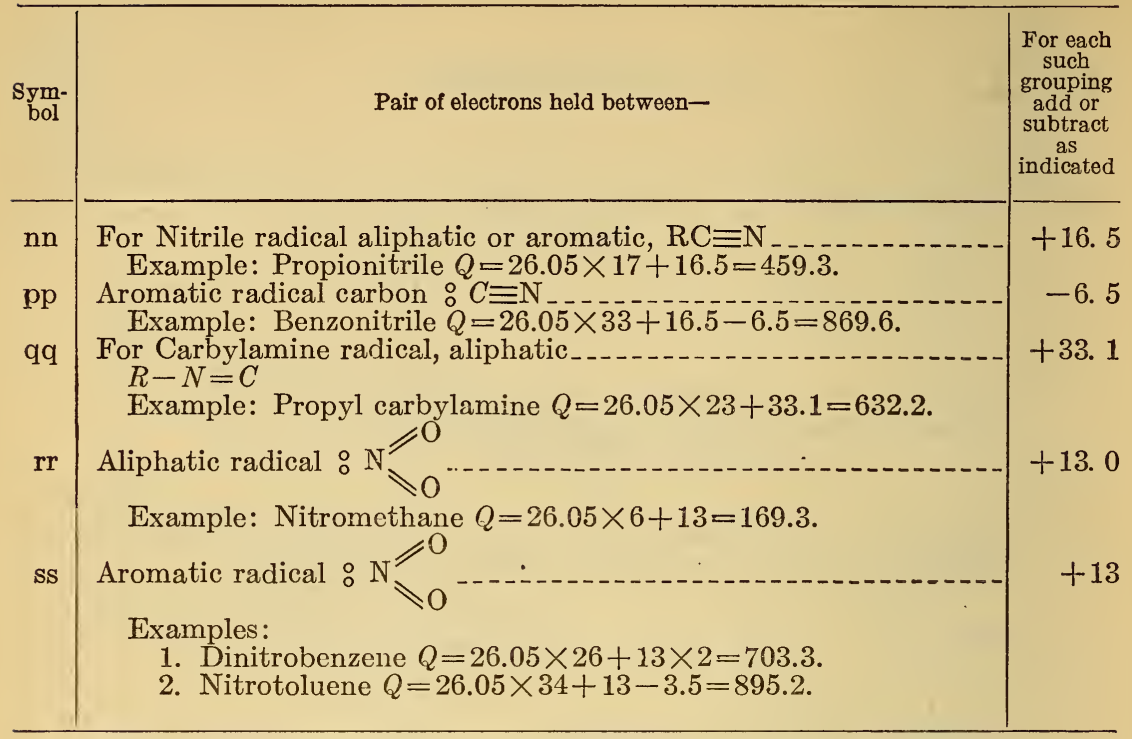

No equations are given for the fluorine, chlorine, and sulphur compounds. However, an examination of the data reveals that the general form of the equation $Q=26.05 \times N+$ a holds fairly well, and it is only necessary to evaluate " $a$ " to obtain the equations for these substances. In the case of sulphur compounds it is also necessary to use a factor different from 26.05 for the sulphur atoms which are burned to $\mathrm{SO}_{2}$ or $\mathrm{SO}_{3}$.

\section{INDEX OF COMPOUNDS, BY CLASSES}

The compounds recorded in the tables are classified in accordance with the distinct types of organic molecules which they represent. The calculation of the heats of combustion is thus facilitated. ${ }^{16}$

\section{1. $\mathrm{CH}$ COMPOUNDS}

1. Saturated hydrocarbons (aliphatic)

2. Polymethylenes_._._. 374

3. Aromatic hydrocarbons

4. Aromatic hydrocarbons (two or more aromatic nuclei linked together)_ $\quad 375$

5. Unsaturated compounds (aliphatic-ethylene)

6. Unsaturated hydrocarbons (aromatic) _._. 376

7. Hydroaromatic hydrocarbons (unsaturated)

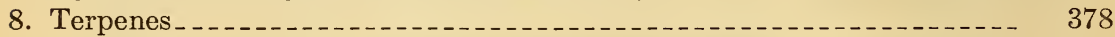

9. Acetylene hydrocarbons

${ }^{16}$ A substance such as cinnamic acid would in this classification come under the heading of unsaturated aliphatic acid. Other similar substances were classified in the samo manner. 


\section{CHO COMPOUNDS}

10. Primary alcohols

11. Primary alcohols (cyclic)

12. Secondary alcohols........ 379

13. Hydroaromatic and polymethylene secondary alcohols__._._._. 380

14. Tertiary alcohols_._._. 380

15. Polyhydroxy aliphatic alcohols

16. Hydroaromatic and polymethylene glycols (all types) _... 381

17. Phenols _._._. 382

18. Aliphatic ethers

19. Aliphatic acetals

20. Aromatic ethers

21. Ethylene oxides ( $\alpha$-oxides)

22. Aliphatic aldehydes_......... 334

23. Aromatic aldehydes._._.

24. Aliphatic ketones.

25. Aromatic ketones_._._. 386

26. Quinones_._.

27. Hydroaromatic and polymethylene ketones_._.

28. Carbohydrates (monosaccharides)

29. Disaccharides _............ 388

30. Trisaccharides, tetrasaccharides, polysaccharides_._._._._. 389

31. Aliphatic acids (saturated monobasic)

32. Aliphatic acids (hydroxy and keto acids) _....... 390

33. Aliphatic acids (unsaturated)

34. Aliphatic acids (monobasic) (acetylene type) 390

35. Aliphatic acids (polybasic saturated)

36. Hydroxy polybasic acids (saturated)

37. Polybasic aliphatic acids (unsaturated)

38. Aromatic acids........... 393

39. Hydroxy aromatic acids 394

40. Phenylated aliphatic acids._._. 394

41. Polybasic aromatic acids...... 395

42. Phenylated polybasic aliphatic acids_._. 396

43. Hydroaromatic and polymethylene acids_._.

44. Acid anhydrides. 397

45. Lactones_._._. 399

46. Methyl esters of monobasic acids_._. 399

47. Methyl esters of monobasic aromatic acids_._. 400

48. Methyl esters of polybasic aliphatic acids._._. 400

49. Methyl esters of polybasic aromatic acids.... 401

50. Ethyl esters of monobasic aliphatic acids_... 401

51. Ethyl esters of monobasic aromatic acids

52. Ethyl esters of polybasic aliphatic acids

53. Esters of aliphatic acids (all types)

54. Esters of aromatic acids (all types)

55. Phenol esters

56. Glycerol esters.

\section{3. $\mathrm{N}$ COMPOUNDS}

57. Aliphatic amines (primary)

58. Aliphatic amines (secondary)

59. Aliphatic amines (tertiary)

60. Aromatic amines (primary) 405

61. Aromatic amines (secondary) 405 
62. Aromatic amines (tertiary)

63. Amino acids (aliphatic)

405

405

64. Amino acids (containing a phenyl radical)

406

65. Amides (aliphatic)

406

66. Acid amides (aromatic)

67. Cyclic ureides, hydantoins, pyrimidines, purines.....

68. Ring nitrogen compounds......

69. Ring nitrogen compounds, imides..........

70. Alkaloids (pyridine, piperidine, quinoline, and isoquinoline)

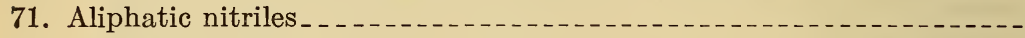

72. Aromatic nitriles

73. Carbylamines

74. Isocyanates (aliphatic)

75. Hydroxylamine derivatives .

76. Aliphatic nitro compounds

77. Aromatic nitro compounds._.

78. Substituted aromatic nitro compounds _.

79. Aromatic nitrophenols, phenetoles, nitranilines, nitroacetanilides

80. Aromatic nitroaldehydes _.

81. Aromatic nitro acids _...

82. Phenylhydrazones and osazones

83. Aldoximes (aliphatic)

84. Ketoximes (aliphatic)

85. Aromatic aldoximes and ketoximes .

86. Nitrosamines_._.

87. Nitramines.

88. Nitroso compounds

89. Azo compounds

90. Substituted hydrazines

91. Diazonium compounds

92. Azoxy compounds

93. Esters of nitric and nitrous acid

407

408

408

409

409

409

410

410

411

411

411

411

412

412

413

413

413

413

413

414

414

414

414

414

415

415

415

415

\section{HALOGEN AND SULPHUR COMPOUNDS}

94. Fluorine compounds:

(a) Fluoro-hydrocarbons (aliphatic)

(b) Fluoro-hydrocarbons (aromatic)

(c) Fluoro-alcohols

(d) Fluoro-phenols

(e) Esters of fluoro-alcohols

(f) Ethers of fluoro-alcohols

(g) Ethers of fluoro-phenols......

(h) Fluoro-acids (aliphatic)

(i) Fluoro-acids (aromatic)

(j) Esters of fluoro-acids (aliphatic)

(k) Esters of fluoro-acids (aromatic)

(l) Eluoro-amides (aciphatic)

(m) Fluoro-amides (aromatic)

(n) Fluoro-amines (aliphatic)

(o) Fluoro-nitramines (aliphatic)

(p) Fluoro-nitrobenzenes . .

(q) Fluoro-nitrotoluenes...

(r) Fluoro-anilines.....

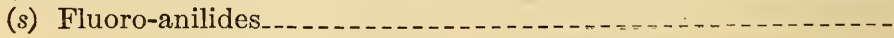

( $t$ ) Fluoro-nitrophenols and nitrophenetoles .

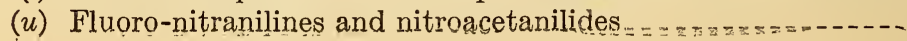


95. Chlorine compounds:

(a) Chlorinated hydrocarbons....... 418

(b) Compounds of $\mathrm{C}, \mathrm{H}, \mathrm{O}$, and $\mathrm{Cl}$. (aliphatic)

(c) Compounds of $\mathrm{C}, \mathrm{H}, \mathrm{O}$, and Cl. (aromatic)

(d) Chloro-hydroquinols..._._. 419

(e) Chloro-quinones......... 419

(f) Chlorine compounds of $\mathrm{C}, \mathrm{H}, \mathrm{N}, \mathrm{O} \ldots$

96. Alkyl bromides_-_- 419

97. Iodine compounds

98. Sulphur compounds._._. 420

\section{TABLES OF DATA}

\section{1. $\mathrm{CH}$ COMPOUNDS}

1. SATURATED HYDROCARBONS (ALIPHATIC)

$Q=26.05 \times N$

\begin{tabular}{|c|c|c|c|c|c|c|c|}
\hline Formula & Name & $\begin{array}{l}\text { Molec- } \\
\text { ular } \\
\text { weight }\end{array}$ & \begin{tabular}{|l} 
Num- \\
ber \\
of \\
elec- \\
trons \\
$(N)$
\end{tabular} & $\begin{array}{l}\mathrm{Kg} \text {-cal } 15 \\
\text { (experi- } \\
\text { mental) }\end{array}$ & $\begin{array}{c}\text { Kilo- } \\
\text { joules } \\
\text { (K. J.) }\end{array}$ & $\begin{array}{c}\mathrm{Kg}-\mathrm{cal}_{15} \\
\text { (calcu- } \\
\text { lated to } \\
\text { the } \\
\text { liquid } \\
\text { state) }\end{array}$ & Literature \\
\hline $\mathrm{CH}_{4--}$ & Methane (g) & 16 & 8 & 1210.8 & 881.6 & 208.4 & 223; cf. 136, \\
\hline $\mathrm{C}_{2} \mathrm{H}_{6-}$ & Ethane (g) & 30 & 14 & ${ }^{2} 368.4$ & $1,540.7$ & 364.7 & 223; cf. 136 \\
\hline $\mathrm{C}_{3} \overrightarrow{\mathrm{H}}_{8-}$ & Propane (g) & 44 & 20 & 3526.3 & $2,201.0$ & 521.0 & 223 ; cf. 136 . \\
\hline $\mathrm{C}_{4} \mathrm{H}_{10-}$ & Isobutane (g) (trimethyl- & 58 & 26 & 4683.4 & $2,858.0$ & 677.3 & 223 . \\
\hline $\mathrm{C}_{5} \mathrm{H}_{12}$ & $n$-Pentane $(\mathrm{g})$ & 72.10 & 32 & 838.3 & $3,511.6$ & 833.6 & 170. \\
\hline $\mathrm{C}_{5} \mathrm{H}_{12-}$ & $n$-Pentane.. & 72.10 & 32 & 833.4 & $3,491.1$ & 833.6 & 170. \\
\hline $\mathrm{C}_{5} \mathrm{H}_{12-}$ & Isopentane $(\mathrm{g})$ & 72. 10 & 32 & 843.5 & $3,533.4$ & 833. 6 & 170. \\
\hline $\mathrm{C}_{5} \mathrm{H}_{12}$ & Isopentane 5 & 72.10 & 32 & 838. 3 & $3,511.6$ & 833. 6 & 170. \\
\hline $\mathrm{C}_{5} \mathrm{H}_{12}$ & Tetramethylmethane (g) & 72.10 & 32 & 6842.6 & $3,523.8$ & 833.6 & 223. \\
\hline $\mathrm{C}_{6} \mathrm{H}_{14-}$ & n-Hexane.. & 86.11 & 38 & $\begin{array}{l}991.4 \\
989.8\end{array}$ & $4,149.0$ & 989.9 & 185. \\
\hline $\mathrm{C}_{6} \mathrm{H}_{14-}$ & Diisopropyl (v). & 86.11 & 38 & 993.9 & $4,156.5$ & 989.9 & 223. \\
\hline $\mathrm{C}_{7} \mathrm{H}_{16-}$ & n-Heptane... & 100.13 & 44 & $\begin{array}{l}1,137.3 \\
1,149.9\end{array}$ & $\begin{array}{l}4,756.2 \\
4,812.3\end{array}$ & $1,146.2$ & $\begin{array}{l}114 . \\
246 .\end{array}$ \\
\hline $\mathrm{C}_{7} \mathrm{H}_{16}$ & 2-Methylhexane.- & 100.13 & 44 & $1,148.9$ & $4,808.1$ & $1,146.2$ & 246. \\
\hline $\mathrm{C}_{7} \mathrm{H}_{16}$ & 3-Methylhexane............... & 100. 13 & 44 & $1,148.9$ & $4,808.1$ & $1,146.2$ & 246 . \\
\hline $\mathrm{C}_{7} \mathrm{H}_{16-}$ & 2,2-Dimethylpentane & 100.13 & 44 & $1,148.9$ & $4,808.1$ & $1,146.2$ & 246. \\
\hline $\mathrm{C}_{7} \mathrm{H}_{16}$ & 2,3-Dimethylpentane & 100.13 & 44 & $1,148.9$ & $4,808.1$ & $1,146.2$ & 246. \\
\hline $\mathrm{C}_{7} \mathrm{H}_{16-}$ & 3,3-Dimethylpentane & 100.13 & 44 & $1,147.9$ & $4,804.0$ & $1,146.2$ & 246. \\
\hline $\mathrm{C}_{7} \mathrm{H}_{16}$ & 2,4-Dimethylpentane. & 100.13 & 44 & $1,148.9$ & $4,808.1$ & $1,146.2$ & 246. \\
\hline $\mathrm{C}_{7} \mathrm{H}_{16}$ & 3-Ethylpentane & 100.13 & 44 & $1,149.9$ & $4,812.3$ & $\mathbf{1}, 146.2$ & 246. \\
\hline $\mathrm{C}_{7} \mathrm{H}_{16-\ldots}$ & 2,2,3-Trimethylbutane_...... & 100.13 & 44 & $\begin{array}{l}1,147.9 \\
1,302.7\end{array}$ & $\begin{array}{l}4,804.0 \\
5,447.9\end{array}$ & $\begin{array}{l}1,146.2 \\
1,302.5\end{array}$ & $\begin{array}{l}246 . \\
215\end{array}$ \\
\hline $\mathrm{C}_{8}$ & n-Octane. & 114.14 & 50 & $1,304.7$ & $5,458.9$ & 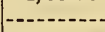 & 154. \\
\hline $\mathrm{C}_{8} \mathrm{~F}$ & 2,5-Dimethylhexane... & 114.14 & 50 & $1,303.3$ & $5,453.0$ & $1,302.5$ & 154. \\
\hline $\mathrm{C}_{8} \mathrm{~F}$ & 2-Methylheptane.... & 114. 14 & 50 & $1,306.1$ & $5,464.7$ & $1,302.5$ & 154. \\
\hline & 3,4-Dimethylhexane.... & 114. 14 & 50 & $1,303.7$ & $5,454.7$ & $1,302.5$ & 154. \\
\hline $\mathrm{C}_{8} \mathrm{H}_{18-}$ & 3-Ethylhexane & 114. 14 & 50 & $1,302.3$ & $5,448.8$ & $1,302.5$ & 154. \\
\hline $\mathrm{C}_{8} \mathrm{H}_{18-}$ & 2,2,4-Trimethylpentane-...-. & 114.14 & 50 & $1,303.9$ & $5,457.0$ & $1,302.5$ & 246. \\
\hline $\mathrm{C}_{8} \mathrm{H}_{18}$ & Hexamethylethane (s) & 114.14 & 50 & $1,301.8$ & $5,448.3$ & $1,302.5$ & 246. \\
\hline $\mathrm{C}_{10} \mathrm{H}_{22}$ & Decane.--- & 142.18 & 62 & $1,610.2$ & $6,733.9$ & $1,615.1$ & 215 \\
\hline $\mathrm{C}_{10} \mathrm{H}_{22}$ & Diisoamyl & 142. 18 & 62 & $1,615.8$ & $6,757.3$ & $1,615.1$ & $153 ;$ cf. 244 \\
\hline $\mathrm{C}_{16} \mathrm{H}_{34}$ & Hexadecane (s) & 226.27 & 98 & $2,559.1$ & $10,709.8$ & $2,552.9$ & 192. \\
\hline $\mathrm{C}_{20} \mathrm{H}_{42--}$ & Eicosane (s) & 282.34 & 122 & $3,183.1$ & $13,321.3$ & $3,178.1$ & 192. \\
\hline
\end{tabular}

1 The above value is the average of 9 determinations which show a maximum variation of 1.1 per cent. 3 The variation between the highest and lowest result equals 1.1 per cent.

' 3 The variation between the highest and lowest result equals 0.9 per cent.

4 The variation between the highest and lowest result equals 0.4 per cent.
5 Note the large difference obtained by (170) for the two isomeric pentanes. This difference is rather unusual, particularly in view of the fact that the 9 isomeric heptanes recorded in this report show almost identical heats of combustion. The heptanes used for this purpose at the Bureau of Standards were of a very high degree of purity. In view of that fact, no great reliance should be attached to the value for isopentane until it is substantiated by other determinations.

8 Value uncertain, since tetramethymethane mixed with butylene was burned. The variation between the highest and lowest result equals 0.2 per cent. 


\section{TABLES OF DATA-Continued}

\section{CH COMPOUNDS-Continued}

\section{POLYMETHYLENES}

$Q=26.05 \times N^{7}$

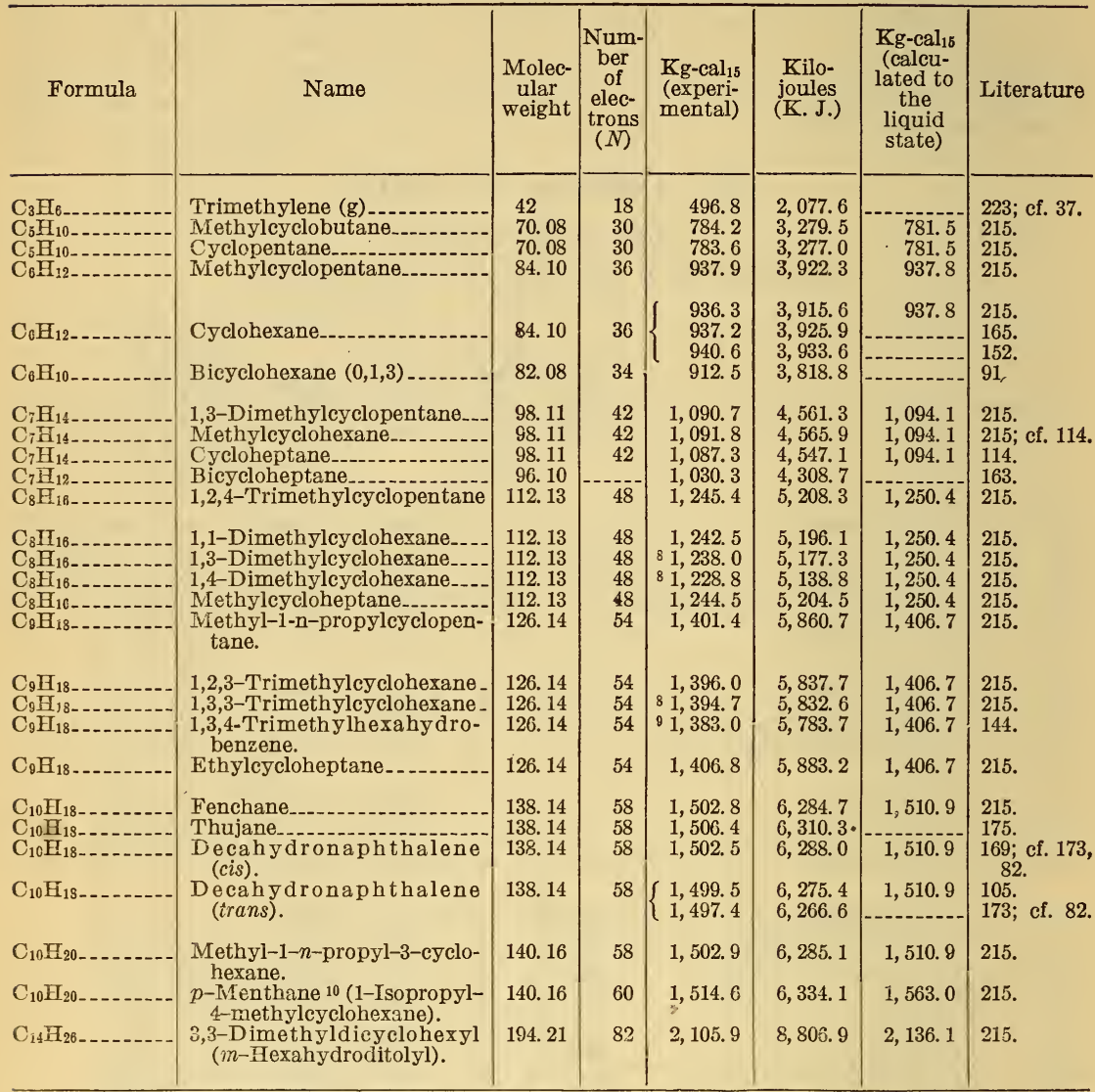

Except for trimethylene and its derivatives and bicyclo compounds containing trimethylene rings.

Swietoslawski (J. Am. Ch. Soc., 42, p. 1315), 1920, believes that the heats of combustion of these compounds are in error and recommends that they be redetermined.

9 This value is probably in error. The work of this investigator does not agree well with the values of modern workers.

10 The author (215) calls the compound "Caromenthane." The heat of combustion of this compound would certainly bear reinvestigation. 


\section{TABLES OF DATA-Continued}

1. CH COMPOUNDS-Continued

\section{AROMATIC HYDROCARBONS}

$Q=26.05 \times N-3.5 \mathrm{a}-6.5 \mathrm{~b}$

\begin{tabular}{|c|c|c|c|c|c|c|c|}
\hline Formula & Name & $\begin{array}{c}\text { Molec- } \\
\text { ular } \\
\text { weight }\end{array}$ & $\begin{array}{l}\text { Num- } \\
\text { ber } \\
\text { of } \\
\text { elec- } \\
\text { trons } \\
(N)\end{array}$ & $\begin{array}{l}\mathrm{Kg}_{\text {-cal }}{ }_{15} \\
\text { (experi- } \\
\text { mental) }\end{array}$ & $\begin{array}{c}\text { Kilo- } \\
\text { joules } \\
\text { (K. J.) }\end{array}$ & $\begin{array}{c}\mathrm{Kg} \text {-cal } \\
\text { (calcu- } \\
\text { lated to } \\
\text { the } \\
\text { liquid } \\
\text { state) }\end{array}$ & Literature \\
\hline $\mathrm{C}_{6} \mathrm{H}_{6} \ldots \ldots$ & Benzene $(\nabla)$ & 78.05 & 30 & 787. 2 & $3,294.4$ & & 200. \\
\hline $\mathrm{C}_{6} \mathrm{H}_{0--}$ & Benzene......... & 78.05 & 30 & $\begin{array}{l}783.4 \\
782.3\end{array}$ & $\begin{array}{l}3,277.7 \\
3,277.1\end{array}$ & 781.5 & 153. \\
\hline $\mathrm{C}_{7} \mathrm{~B}_{8--}$ & Toluene.- & 92.06 & 36 & $\begin{array}{l}937.0 \\
934.2\end{array}$ & $\begin{array}{l}3,920.4 \\
3,913.4\end{array}$ & 934.3 & $\begin{array}{l}152 \text {; cf. } 203 \text {, } \\
153 \text {. }\end{array}$ \\
\hline $\mathrm{C}_{8} \mathrm{H}_{10} \ldots$ & o-Xylene.. & 106.08 & 42 & $\left\{\begin{array}{l}1,094.2 \\
1,091.7\end{array}\right.$ & $\begin{array}{l}4,578.1 \\
4,567.7\end{array}$ & $1,087.1$ & $\begin{array}{l}154 . \\
152 .\end{array}$ \\
\hline $\mathrm{C}_{8} \mathrm{H}_{10-}$ & $m$-Xylene & 106.08 & 42 & $\left\{\begin{array}{l}1,091.7 \\
1,088.4\end{array}\right.$ & $\begin{array}{l}4,567.7 \\
4,559.3\end{array}$ & $1,087.1$ & 165 ; cf. 154 \\
\hline $\mathrm{C}_{8} \mathrm{H}_{10} \ldots$ & $p$-Xylene... & 106.08 & 42 & $1,089.1$ & $4,556.8$ & $1,087.1$ & 154 ; cf. 152 , \\
\hline $\mathrm{C}_{8} \mathrm{H}_{10} \ldots$ & Ethylbenzene................ & 106.08 & 42 & $1,091.2$ & $4,565.6$ & $1,087.1$ & 152; cf. 138 . \\
\hline $\mathrm{C}_{9} \mathrm{H}_{12-}$ & Mesitylene.......... & 120.10 & 48 & $1,243.6$ & $5,203.2$ & $1,239.9$ & 152 ; cf. 203. \\
\hline $\mathrm{C}_{9} \overline{\mathrm{H}}_{12-}$ & n-Propylbenzene........ & 120.10 & 48 & $1,246.4$ & $5,214.9$ & $1,246.9$ & 152 ; cf. 73 \\
\hline $\mathrm{C}_{9} \mathrm{H}_{12-}$ & Isopropylbenzene & 120.10 & 48 & $1,247.3$ & $5,218.7$ & $1,246.9$ & 152 ; cf. 73 . \\
\hline $\mathrm{C}_{9} \mathrm{H}_{12} \ldots$ & $\begin{array}{l}\text { Pseudocumene (1,2,4-Tri- } \\
\text { methylbenzene). }\end{array}$ & $120 . .10$ & 48 & $1,241.7$ & $5,195.3$ & $1,239.9$ & 152. \\
\hline $\mathrm{C}_{10} \mathrm{H}_{1}$ & tert-Butylbenzene.... & 134. 11 & 54 & $1,400.4$ & $5,859.3$ & $1,403.2$ & $152 ;$ cf. 153. \\
\hline $\mathrm{C}_{10} \mathrm{H}_{14-}$ & $\begin{array}{l}\text { 1,2,4,5-Tetramethylbenzene } \\
\text { (s) (Durene). }\end{array}$ & 134. 11 & 54 & $1,393.6$ & $5,832.2$ & $1,392.7$ & 188. \\
\hline $\mathrm{C}_{10} \mathrm{H}_{14 \ldots}$ & $\begin{array}{l}\text { iso-Propyltoluene }(1,4)(\mathrm{Cy}- \\
\text { mene). }\end{array}$ & 134. 11 & 54 & $\left\{\begin{array}{l}1,412.3 \\
1,402.8\end{array}\right.$ & $\begin{array}{l}5,910.5 \\
5,866.5\end{array}$ & $1,399.7$ & 182. \\
\hline $\mathrm{C}_{10} \mathrm{H}_{14}$ & $n$-Propyltoluene $(1,3)$ & 134. 11 & 54 & $121,405.4$ & $5,878.8$ & $1,399.7$ & 73. \\
\hline $\mathrm{C}_{10} \mathrm{H}_{14}$ & Isopropyltoluene $(1,3)$ & 134. 11 & 54 & $121,409.5$ & $5,895.9$ & $1,399.7$ & 73. \\
\hline $\mathrm{C}_{11} \mathrm{H}_{10}$ & Pentamethylbenzene $(\mathrm{s})$ & 148. 13 & 60 & $1,554.0$ & $6,503.5$ & $1,545.5$ & 188. \\
\hline $\mathrm{C}_{12} \mathrm{H}_{18}$ & Hexamethylbenzene (s) & 162. 14 & 66 & $1,711.9$ & $7,164.3$ & $1,700.3$ & 188. \\
\hline $\mathrm{C}_{13} \mathrm{H}_{12-}$ & Diphenylmethane (s) & 168.10 & 64 & $1,655.0$ & $6,926.2$ & $1,660.2$ & $182 ;$ cf. 179. \\
\hline $\mathrm{C}_{14} \mathrm{H}_{14-}$ & Dibenzyl (s) & 182. 11 & 70 & $1,810.6$ & $7,577.4$ & $1,816.5$ & 182. \\
\hline $\mathrm{C}_{19} \mathrm{H}_{15-}$ & Triphenylmethyl (s) ${ }^{13} \ldots$ & 243.12 & 91 & $142,378.5$ & $9,946.9$ & $2,373.5$ & 179. \\
\hline $\mathrm{C}_{19} \mathrm{H}_{16}$ & Triphenylmethane (s). & 244. 13 & 92 & $\left\{\begin{array}{l}2,379.3 \\
2,388.7\end{array}\right.$ & $\begin{array}{l}9,957.4 \\
9,996.9\end{array}$ & $2,386.1$ & $\begin{array}{l}182 . \\
179 .\end{array}$ \\
\hline $\mathrm{C}_{25} \mathrm{H}_{20}$ & Tetraphenylmethane (s) & 320.16 & 120 & $3,102.4$ & $12,974.2$ & $3,112.0$ & 179 . \\
\hline
\end{tabular}

11 The authors (4) report the heats of combustion of two samples of ethylbenzene prepared by the Fittig and Clemensen methods, respectively. The calorimetric determinations were carried out by Langbein and agree within 0.1 per cent with the value recorded by (152). Of interest is the fact that, while the two samples have almost identical heats of combustion within 0.2 per cent, the sample obtained by the Clemensen method has the higher density and index of refraction.

12 The values of this investigator are about 0.4 per cent too high as compared with those of Richards and Barry (16).

13 The author (179) gives, also, the heat of combustion of triphenylmethyl peroxide and the heat of combustion of the addition product of hexaphenylethane and ethyl acetate.

14 This molecule contains one displaced electron; hence the formula for it becomes $26.05 \times N+13=2,373.5$.

\section{AROMATIC HYDROCARBONS}

(Two or more aromatic nuclei linked together)

$Q=26.05 \times N-3.5 \mathrm{a}-6.5 \mathrm{~b}$

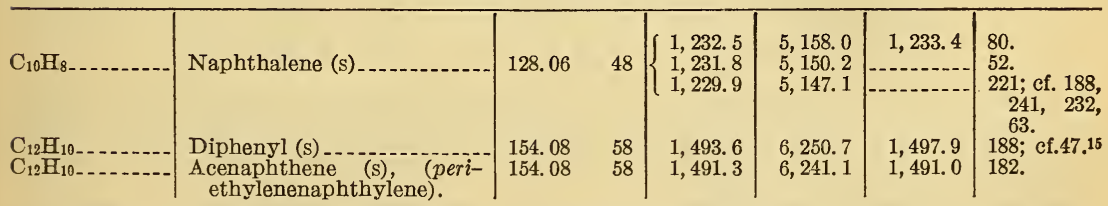

15 These authors give the heats of combustion of a freshly prepared sample of diphenyl and one 20 years old, the difference being $11.2 \mathrm{~kg}$-cal $\mathrm{l}_{15}$ per mole. The authors accept the higher value of $1,510.1 \mathrm{~kg}-\mathrm{cal} \mathrm{l}_{15}$ for the fresh sample as the correct value and believe that the new sample is of a higher degree of purity than the old sample.

$$
3697^{\circ}-29-2
$$




\section{TABLES OF DATA-Continued}

\section{CH COMPOUNDS-Continued}

\section{AROMATIC HYDROCARBONS-Continued}

(Two or more aromatic nuclei linked together)

$Q=26.05 \times N-3.5 \mathrm{a}-6.5 \mathrm{~b}$

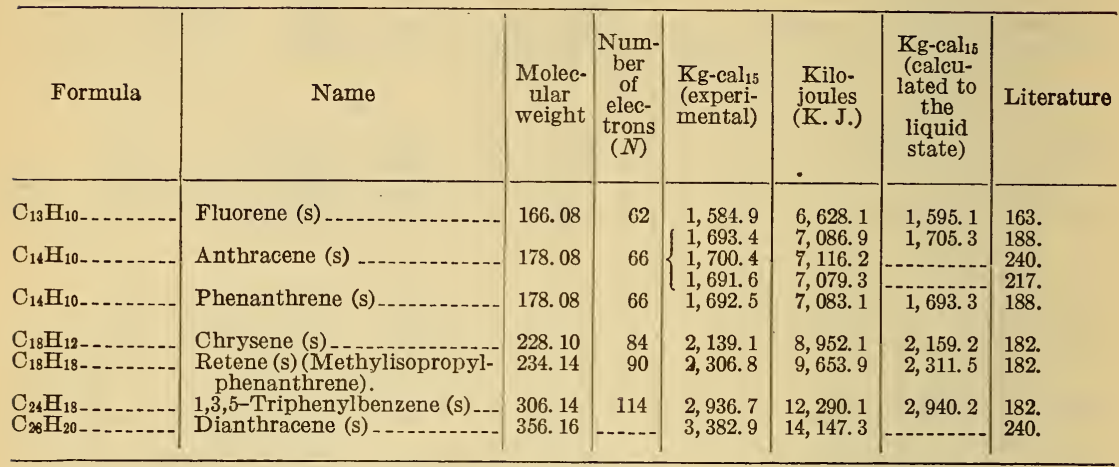

\section{UNSATURATED COMPOUNDS}

(Aliphatic-ethylene)

$Q=26.05 \times N+13$

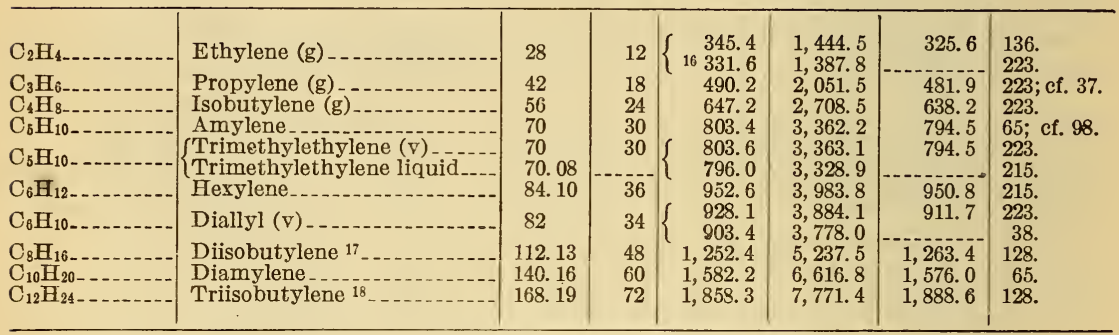

16 Thomsen's value is probably more reliable than the higher value of Mixter.

$17\left(\mathrm{CH}_{3}\right)_{2} \mathrm{CH} . \mathrm{CH}: \mathrm{CH} . \mathrm{CH} .\left(\mathrm{CH}_{3}\right)_{2}$.

$18\left(\mathrm{CH}_{3}\right)_{2} . \mathrm{CH} . \mathrm{C}\left[\mathrm{CH}_{2} . \mathrm{CH} .\left(\mathrm{CH}_{3}\right)_{2}\right]: \mathrm{CH} . \mathrm{CH}\left(\mathrm{CH}_{3}\right)_{2}$.

\section{UNSATURATED HYDROCARBONS}

(Aromatic)

$Q=26.05 \times N+13-6.5 \mathrm{~d}$

\begin{tabular}{|c|c|c|c|c|c|c|c|}
\hline $\mathrm{C}_{8} \mathrm{H}_{8}$ & Styrene (Phenylethylene) ..-- & 104.06 & 40 & $\left\{\begin{array}{l}1,045.4 \\
1,047.1\end{array}\right.$ & $\begin{array}{l}4,375.0 \\
4,386.3\end{array}$ & $1,048.5$ & $\begin{array}{l}182 . \\
7 \text {; cf. } 138,\end{array}$ \\
\hline $\mathrm{C}_{0} \mathrm{H}_{10}$. & $\alpha$-Methylstyrene & 118.08 & 46 & 1, 202. 9 & 5, & 1,2 & $7 ;$ cf. 104 \\
\hline $10 \mathrm{H}_{12}$ & 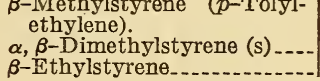 & $\begin{array}{l}118.08 \\
132.10 \\
132.10\end{array}$ & $\begin{array}{l}52 \\
52\end{array}$ & $\begin{array}{l}1,202.4 \\
1,357.2 \\
1,346.1\end{array}$ & $\begin{array}{l}5,685.3 \\
5,638.8\end{array}$ & $\begin{array}{l}1,204.8 \\
1,361.1 \\
1,361.1\end{array}$ & 7. cf. 7 . \\
\hline
\end{tabular}

19 The author (104) gives values for styrene and substitution products of that compound which are about 1.4 per cent higher than the values given by (7). As the details of the former (8) work are entirely lacking, and since, in general, the work of (7) is most painstaking, we may safely assume that the values of (104) are in error, and that his results are at least 0.8 per cent too high. No greater accuracy than 0.3 to 0.5 per cent can even then be attached to his values.

${ }_{20}$ Recently Swietoslawski and Popov (J. chim. phys., 22, p. 397; 1925) have attempted to correct Lemoult's values by introducing a correction of -0.5 per cent. While it is possible that the result of this investigator contains a systematic error, yet we do not believe that this correction brings all of Lemoult's values into agreement with those of later investigators, which indicates other sources of error. In the tables the corrected values are recorded. 


\section{TABLES OF DATA-Continued}

\section{CH COMPOUNDS-Continued}

\section{UNSATURATED HYDROCARBONS-Continued}

(Aromatic)

$Q=26.05 \times N+13-6.5 \mathrm{~d}$

\begin{tabular}{|c|c|c|c|c|c|c|c|}
\hline Formula & Name & $\begin{array}{c}\text { Molec- } \\
\text { ular } \\
\text { weight }\end{array}$ & $\begin{array}{c}\text { Num- } \\
\text { ber } \\
\text { of } \\
\text { elec- } \\
\text { trons } \\
(N)\end{array}$ & $\begin{array}{l}\text { Kg-cal } 15 \\
\text { (experi- } \\
\text { mental) }\end{array}$ & $\begin{array}{l}\text { Kilo- } \\
\text { joules } \\
\text { (K. J.) }\end{array}$ & $\begin{array}{l}\mathrm{Kg}-\mathrm{cal}_{15} \\
\text { (calcu- } \\
\text { lated to } \\
\text { the } \\
\text { liquid } \\
\text { state) }\end{array}$ & Literature \\
\hline $\mathrm{C}_{10} \mathrm{H}_{12} \ldots$ & Phenyl-1-butene-2...... & 132.10 & 52 & $1,361.2$ & $5,702.1$ & $1,364.1$ & 7. \\
\hline $\mathrm{C}_{10} \mathrm{H}_{12-}$ & Phenyl-1-butene-3.... & 132.10 & 52 & $1,356.9$ & $5,675.9$ & $1,364.1$ & 138. \\
\hline $\mathrm{C}_{11} \mathrm{H}_{14--}$ & Phenyl-1-pentene-2.- & 146. 11 & 58 & $1,510.0$ & $6,325.4$ & $1,517.4$ & \\
\hline $\mathrm{C}_{12} \mathrm{H}_{16} \ldots$. & $\beta, \beta$-Diethylstyrene & 160.13 & 64 & $1,664.9$ & $6,974.3$ & $1,673.7$ & $6 ;$ cf. 7 . \\
\hline $\mathrm{C}_{14} \mathrm{H}_{12} \ldots$ & $\begin{array}{l}\text { Stilbene (s) (symm. Diphen- } \\
\text { ylethylene). }\end{array}$ & 180.10 & 68 & $1,765.0$ & $7,381.2$ & $1,771.4$ & $\begin{array}{l}183 ; \text { cf. } 163 \text {, } \\
144,9 .\end{array}$ \\
\hline $\mathrm{C}_{14} \mathrm{H}_{12-}$ & Isostilbene & 180.10 & 68 & $1,770.9$ & $7,405.9$ & $1,771.4$ & 9. \\
\hline $\mathrm{C}_{15} \mathrm{H}_{14} \ldots$ & $\alpha, \beta$-Methylphenylstyrene (s) & 194.11 & 74 & $211,937.9$ & $8,106.2$ & $1,927.7$ & 104. \\
\hline $\mathrm{C}_{16} \mathrm{H}_{14 \ldots}$ & $\begin{array}{l}\text { Diphenylbutadiene (s) (la- } \\
\text { bile). }\end{array}$ & 206.11 & 78 & $2,056.9$ & $8,608.1$ & $2,044.9$ & 172. \\
\hline $\mathrm{C}_{16} \mathrm{H}_{14--}$ & $\begin{array}{l}\text { Diphenylbutadiene (s) (cis- } \\
\text { cis). }\end{array}$ & 206.11 & 78 & $2,035.1$ & $8,516.9$ & $2,044.9$ & 172. \\
\hline $\mathrm{C}_{16} \mathrm{H}_{14-}$ & $\begin{array}{l}\text { Diphenylbutadiene(s) (trans- } \\
\text { trans). }\end{array}$ & 206.11 & 78 & $2,030.3$ & $8,496.8$ & $2,031.9$ & 172.22 \\
\hline $\mathrm{C}_{18} \mathrm{H}_{16}$ & Diphenylhexatriene $(\delta)^{23} \ldots$ & 232.13 & 88 & $2,287.7$ & $9,574.0$ & $2,318.4$ & 172. \\
\hline $\mathrm{C}_{18} \overline{\mathrm{H}}_{18-}$ & $\begin{array}{l}\text { 1, 6-Diphenylhexadiene-1, } 5 \\
\text { (s). }\end{array}$ & 234.14 & 90 & $2,342.2$ & $9,811.5$ & $2,355.5$ & 163. \\
\hline $\mathrm{C}_{18} \mathrm{H}_{18-}$ & Dibenzylbutadiene (s) & 234.14 & 90 & $2,341.0$ & $9,797.1$ & $2,357.5$ & 172. \\
\hline $\mathrm{C}_{18} \mathrm{H}_{20}$ & $\begin{array}{l}\text { Diphenyl-1, 4-ethyl-1-bu- } \\
\text { tene-3. }\end{array}$ & 24236.15 & 92 & $2,372.6$ & $9,939.3$ & $2,399.6$ & 7. \\
\hline $\mathrm{C}_{20} \mathrm{H}_{16}$ & Diphenylstyrene (s) & 256.13 & 96 & ${ }^{25} 2,508.6$ & $10,493.5$ & $2,494.3$ & 104. \\
\hline
\end{tabular}

21 This value is about 0.6 per cent too high. A better value would be $1,929.2$ See footnotes 19 and $20^{\circ}$ p. 376.

${ }_{22}$ For trans isomers the correction is 6.5 for the double bond. Consult p. 363 .

23 The purity of this product is rather questionable.

${ }_{24} \mathrm{C}_{6} \mathrm{H}_{5} . \mathrm{CH}\left(\mathrm{CH}_{2} . \mathrm{CH}_{3}\right) \cdot \mathrm{CH}_{2} . \mathrm{CH}=\mathrm{CH}_{2} \mathrm{C}_{6} \mathrm{H}_{5}$.

25 The values of this investigator for this series are uniformly too high by 0.5 per cent. A better value would be $2,496.1 \mathrm{~kg}$-cal 15 .

\section{HYDROAROMATIC HYDROCARBONS 26}

\section{(Unsaturated)}

$Q=26.05 \times N+6.5 \mathrm{e}$

\begin{tabular}{|c|c|c|c|c|c|c|c|}
\hline $\mathrm{C}_{6} \mathrm{H}_{8} \ldots \ldots \ldots$ & Dihydrobenzene.............. & 80.06 & 32 & $\begin{array}{l}833.2 \\
847.8\end{array}$ & $\begin{array}{l}3,484.4 \\
3,548.0\end{array}$ & 846.6 & $\begin{array}{l}215 . \\
197 .\end{array}$ \\
\hline $\mathrm{C}_{6} \mathrm{H}_{10} \ldots \ldots$ & $\begin{array}{l}\text { Dimeth ylmethylenecyclo- } \\
\text { propane. }\end{array}$ & 82.08 & 34 & 898.0 & $3,755.4$ & & 215. \\
\hline $\mathrm{C}_{6} \mathrm{H}_{10 \ldots \ldots} \ldots$ & $\begin{array}{l}\text { Tetrahydrobenzene (cyclo- } \\
\text { hexene). }\end{array}$ & 82.08 & 34 & $\left\{\begin{array}{l}891.2 \\
893.7 \\
891.9\end{array}\right.$ & $\begin{array}{l}3,727.0 \\
3,743.7 \\
3,732.6\end{array}$ & $\begin{array}{r}892.2 \\
\end{array}$ & $\begin{array}{l}215 . \\
165 . \\
197 ; \text { cf. } 91.37\end{array}$ \\
\hline $\mathrm{C}_{7} \mathrm{H}_{12 \ldots} \ldots$ & Methyl-1-cyclohezene-3....- & 96.10 & 40 & $1,043.6$ & $4,364.3$ & $1,048.5$ & 215 \\
\hline $\mathrm{C}_{7} \mathrm{H}_{12--}$ & Methyl-1-cyclohexene-1. & 96.10 & 40 & $\begin{array}{l}1,040.9 \\
1,048.1\end{array}$ & $\begin{array}{l}4,353.0 \\
4,390.5\end{array}$ & $1,048.5$ & $\begin{array}{l}215 . \\
165 ; \text { cf. } 162 .\end{array}$ \\
\hline $\mathrm{C}_{7} \mathrm{H}_{12}-$ & Methylenecyclohezane & 96.10 & 40 & $\begin{array}{l}1,044.1 \\
1,051.4 \\
1,054.9\end{array}$ & $\begin{array}{l}4,366.4 \\
4,404.3 \\
4,414.8\end{array}$ & $\begin{array}{c}1,055.0 \\
-\end{array}$ & $\begin{array}{l}215 . \\
163 . \\
91 .\end{array}$ \\
\hline $\mathrm{C}_{7} \mathrm{H}_{12}--$ & Cycloheptene & 96.10 & 40 & $1,049.9$ & $4,390.7$ & $1,048.5$ & 215. \\
\hline $\mathrm{C}_{8} \mathrm{H}_{12}$ & $\begin{array}{l}\text { 1, Methyl-3-methylenecyclo- } \\
\text { hexene-1. }\end{array}$ & 108.10 & 44 & $1,149.2$ & 4.0 & $1,165.7$ & 162. \\
\hline $\mathrm{C}_{8} \mathrm{H}_{12 \ldots} \ldots$ & 1,3-Dimethyldihydroben- & 108.10 & 44 & $281,148.2$ & $4,801.7$ & $1,159.2$ & 215. \\
\hline $\mathrm{C}_{8} \mathrm{H}_{12-}$ & $\begin{array}{l}\text { 1,4-Dimethylcyclohexadi- } \\
\text { ene- } 1,3 \text {. }\end{array}$ & 108. 10 & 44 & $1,152.2$ & $4,826.6$ & $1,159.2$ & 165. \\
\hline $\mathrm{C}_{8} \mathrm{H}_{1}$ & Ethyl-1-cyclohexene-1....-. & 110.11 & 46 & $1,203.7$ & $5,042.3$ & $1,204.8$ & 165 ; cf. 162. \\
\hline
\end{tabular}

${ }^{26}$ In the case of 1,4 conjugated systems, the correction factor for each double bond should be not 13 , but a smaller value, say 6.5 , in agreement with the lower reactivity of these compounds. It is, however, omitted here, for the data at hand do not allow one to draw far-reaching conclusions and are too conflicting.

${ }_{27}$ This author gives the heat of combustion of cyclohexene as $898.8 \mathrm{~kg}$.-cal ${ }_{15}$ Whether it is for constant volume or constant pressure he does not state, and he gives no experimental details of any kind except that he used the internationally accepted value for benzoic acid.

23 Compare values obtained by (215) and (197) for dihydrobenzene. 


\section{TABLES OF DATA-Continued}

1. CH COMPOUNDS-Continued

7. HYDROAROMATIC HYDROCARBONS-Continued

(Unsaturated)

$Q=26.05 \times N+6.50$

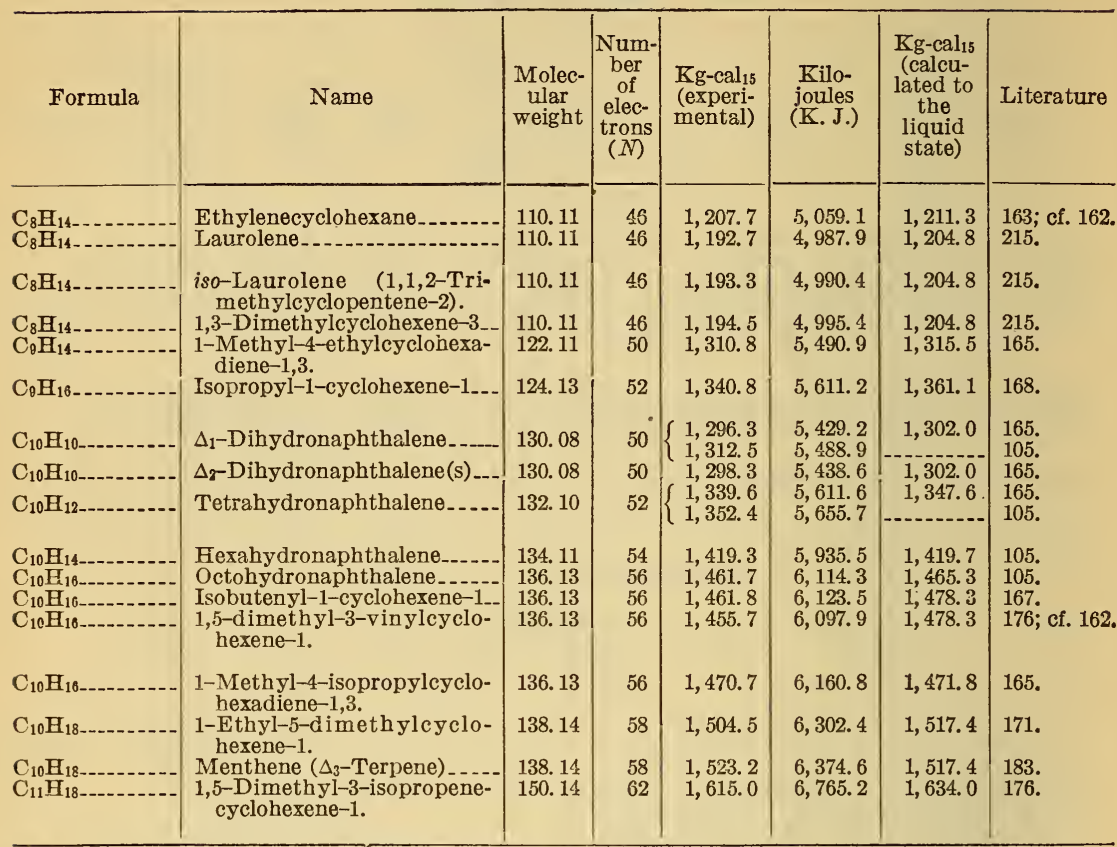

8. TERPENES

$Q=26.05 \times N+13 \mathrm{c}+6.5 \mathrm{e}$

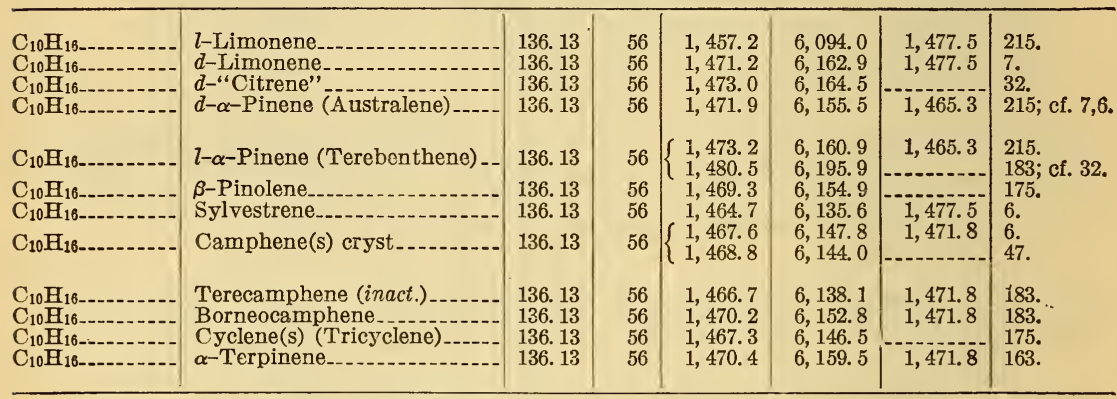

\section{ACETYLENE HYDROCARBONS}

$\mathrm{Q}=26.05 \times N+33.1 \mathrm{~h}+46.1 \mathrm{~g}$

\begin{tabular}{|c|c|c|c|c|c|c|c|}
\hline $\mathrm{C}_{2} \mathrm{H}_{2--}$ & Acetylene (g) (Ethine) & 26.02 & 10 & ${ }^{2 \theta} 312.0$ & $1,304.8$ & 306.1 & 137; cf. 223, \\
\hline $\mathrm{C}_{3} \mathrm{H}_{4--}$ & Allylene (g) (Propine) & 40 & 16 & $\begin{array}{l}465.1 \\
473.0\end{array}$ & $\begin{array}{l}1,945.5 \\
1,978.6\end{array}$ & 462.9 & 223. 103 \\
\hline $\mathrm{C}_{6} \mathrm{H}_{0-}$ & $\begin{array}{l}\text { Dipropargyl (v) (1,5-Hexa- } \\
\text { diine). }\end{array}$ & 78 & 30 & 853.5 & $3,570.2$ & 873.7 & 12. \\
\hline $\mathrm{C}_{6} \mathrm{H}_{6}$ & Dimethyldiacetylene $\quad(2,4-$ & 78.05 & 30 & 847.8 & $3,546.4$ & 847.7 & 122. \\
\hline $\begin{array}{l}\mathrm{C}_{7} \mathrm{H}_{12} \\
\mathrm{C}_{8} \mathrm{H}_{6} \ldots\end{array}$ & Heptine-1 & $\begin{array}{r}96.10 \\
102.05\end{array}$ & $\begin{array}{l}40 \\
38\end{array}$ & $\begin{array}{l}1,091.2 \\
1,024.2\end{array}$ & $\begin{array}{l}4,564.5 \\
4,284.2\end{array}$ & $\begin{array}{l}1,088.0 \\
1,029.5\end{array}$ & $\begin{array}{l}138 . \\
138 .\end{array}$ \\
\hline $\begin{array}{l}\mathrm{C}_{10} \mathrm{H}_{10} \\
\mathrm{C}_{10} \mathrm{H}_{10}\end{array}$ & $\begin{array}{l}\text { ethine). } \\
\text { Phenyl-1-butine-3 } \\
\text { Diphenyldiacetylene (s) -- }\end{array}$ & $\begin{array}{l}130.08 \\
202.08\end{array}$ & $\begin{array}{l}50 \\
74\end{array}$ & $\begin{array}{l}1,340.0 \\
1,975.6\end{array}$ & $\begin{array}{l}5,605.2 \\
8,267.9\end{array}$ & $\begin{array}{l}1,342.1 \\
1,980.9\end{array}$ & $\begin{array}{l}138 . \\
172 .\end{array}$ \\
\hline
\end{tabular}

20 The author (137) gives also the heat of explosion of acetylene. 


\section{TABLES OF DATA-Continued}

2. CHO COMPOUNDS

10. PRIMARY ALCOHOLS

$Q=26.05 \times N+13$

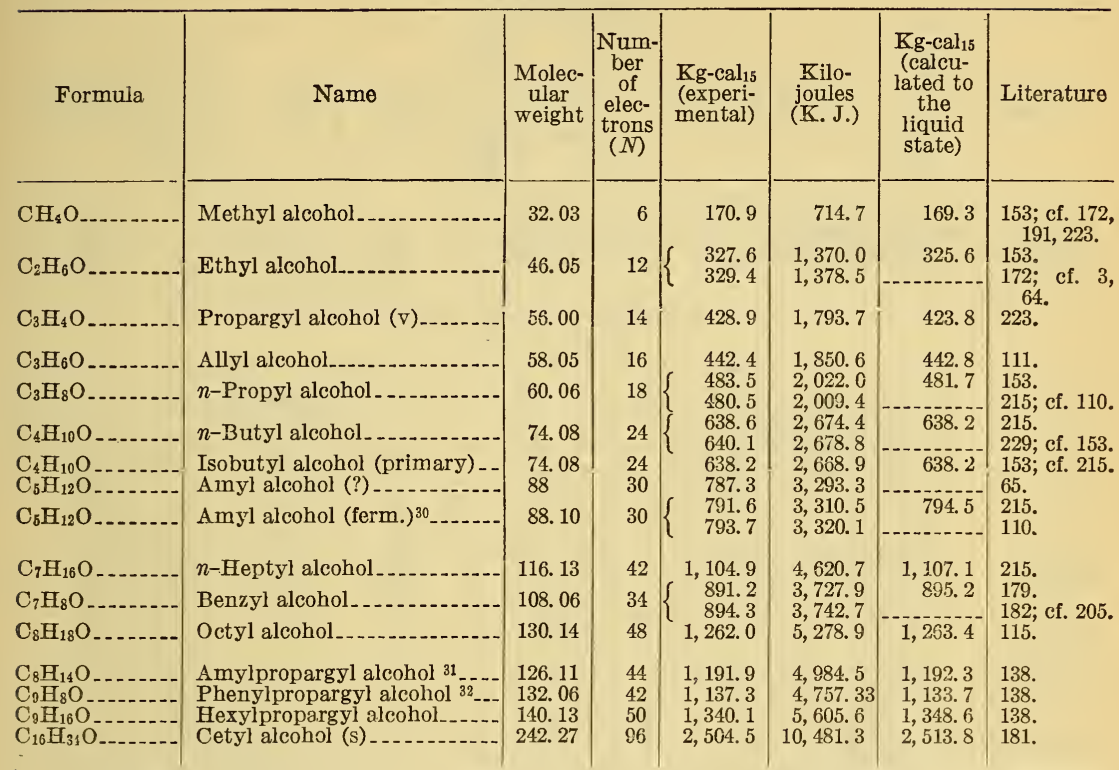

${ }^{30}$ Mostly isobutyl carbinol. $\quad{ }_{31} \mathrm{C}_{5} \mathrm{H}_{11} . \mathrm{C} \equiv \mathrm{C}-\mathrm{CH}_{2} \mathrm{OH}$.

${ }^{32} \mathrm{C}_{6} \mathrm{II}_{5} \mathrm{C} \equiv \mathrm{C}-\mathrm{CH}_{2} \mathrm{OH}$.

11. PRIMARY ALCOHOLS (Cylic) ${ }^{33}$

$Q=26.05 \times N+13$

\begin{tabular}{|c|c|c|c|c|c|c|c|}
\hline $\mathrm{C}_{6} \mathrm{H}_{10} \mathrm{O} \ldots$ & $\begin{array}{l}\text { Cyclobutyl carbinol } \\
\text { Cyclohexyl carbinol. }\end{array}$ & $\begin{array}{r}86.08 \\
114.11\end{array}$ & $\begin{array}{l}28 \\
40\end{array}$ & $\begin{array}{r}747.8 \\
1,047.2\end{array}$ & $\begin{array}{l}3,127.3 \\
4,379.4\end{array}$ & $\begin{array}{r}742.4 \\
1,055.0\end{array}$ & $\begin{array}{l}215 . \\
215 .\end{array}$ \\
\hline
\end{tabular}

${ }^{33}$ Among the cyclic alcohols should be included cholesterol. However, it is omitted here because Berthelot and André, Ann. chim. phys., (7), 17, p. 433; 1899, who determined the heat of combustion of cholesterol, give for the formula of the compound $\mathrm{C}_{26} \mathrm{H}_{44} \mathrm{O}$. The present accepted formula is $\mathrm{C}_{27} \mathrm{H}_{46} \mathrm{O}$.

12. SECONDARY ALCOHOLS

$Q=26.05 \times N+6.5$

\begin{tabular}{|c|c|c|c|c|c|c|c|}
\hline $\mathrm{C}_{3} \mathrm{H}_{8} \mathrm{O}_{-}$ & Isopropyl alcohol. & 60.06 & 18 & 474.8 & $1,985.6$ & 475.4 & 215; cf. 110. \\
\hline $\mathrm{C}_{5} \mathrm{H}_{10} \mathrm{O}_{-}$ & Ethylvinyl carbinol. & 86.08 & 28 & 752.9 & $3,148,6$ & 748.9 & 111. \\
\hline $\mathrm{C}_{0} \mathrm{H}_{14} \mathrm{O}_{-}$ & Pinacolyl alcohol & 102.11 & 36 & 938.6 & $3,925.2$ & 944.3 & 215. \\
\hline $\mathrm{C}_{7} \mathrm{H}_{12} \mathrm{O}$ & Diallyl carbinol. & 112.10 & 38 & $1,028.2$ & $4,299.9$ & $1,022.4$ & 215. \\
\hline $\mathrm{C}_{13} \mathrm{H}_{12} \mathrm{O}$ & Diphenyl carbinol (s) & 184.10 & 62 & $1,615.4$ & $6,760.5$ & $1,614.6$ & 182 ; cf. 179 . \\
\hline $\mathrm{C}_{14} \mathrm{H}_{18} \mathrm{O}_{-}$ & $\begin{array}{l}\text { Amylphenylpropargyl alco- } \\
\text { hol.34 }\end{array}$ & 202. 14 & 72 & $1,901.1$ & $7,910.4$ & $1,921.7$ & 138. \\
\hline $\mathrm{C}_{5} \mathrm{H}_{10} \mathrm{O}_{2}$ & Dimethylolcyclopropane.... & 102.08 & & 707.6 & $2,959.2$ & & 215. \\
\hline $\mathrm{C}_{6} \mathrm{H}_{12} \mathrm{O}$ & $\beta-$ Methylcyclopentanol.. & 100. 10 & 34 & 887.6 & $3,711.9$ & 892.7 & 215. \\
\hline $\mathrm{C}_{6} \mathrm{H}_{12} \mathrm{O}$ & Cyclohexanol & 100.10 & 34 & 890.7 & $3,724.9$ & 892. 7 & 153 ; cf. 215. \\
\hline $\mathrm{C}_{7}$ & 1,3-Dimethylcyclopentanol-2 & 114. 11 & 40 & $1,030.5$ & $4,309.6$ & $1,048.5$ & 215. \\
\hline $\mathrm{C}_{7} \mathrm{H}_{14} \mathrm{O}$ & Ethyl-1-cyclopentanol-2. & 114.11 & 40 & $1,039.0$ & $4,345.1$ & $1,048.5$ & 215. \\
\hline $\mathrm{C}_{7} \mathrm{H}_{14}$ & $\beta$-Methylcyclohexanol & 114.11 & 40 & $1,038.4$ & $4,342.6$ & $1,048.5$ & 215. \\
\hline & Cycloheptanol & 114.11 & 40 & $1,050.2$ & $4,391.9$ & $1,048.5$ & 215. \\
\hline & 1-3-Dimethylcyclohexanol-2. & 128.13 & 46 & $1,196.0$ & $5,001.7$ & $1,204.8$ & 215. \\
\hline $\mathrm{C}_{8} \mathrm{H}_{16} \mathrm{O}$ & 1,3-Dimethylcyclohexanol-5_ & 128.13 & 46 & $1,183.4$ & $4,949.0$ & $1,204.8$ & 215. \\
\hline $\mathrm{C}_{2} \mathrm{H}_{18} \mathrm{O}$ & Cycloheptylmethyl carbinol_ & 142.14 & 52 & $1,342.2$ & $5,613.1$ & $1,361.1$ & 215. \\
\hline $\mathrm{C}_{10} \mathrm{H}_{18} \mathrm{O}$ & Thujyl alcohol & 154. 14 & 56 & $1,477.5$ & $6,189.3$ & & 175. \\
\hline & Borneol (Borneo camphor) & 154.14 & 56 & $351,469.6$ & $6,145.9$ & $1,465.3$ & 125. \\
\hline $\mathrm{C}_{10} \mathrm{H}_{18} \mathrm{O}$ & Borneol (synthetic) & 154.14 & 56 & $1,466.2$ & $6,136.1$ & $1,465.3$ & $183 ;$ cf. 125 \\
\hline & d-Borneol (s) & 154. 14 & 56 & $1,466.6$ & $6,131.9$ & $1,465.3$ & 89. \\
\hline $\mathrm{C}_{10} \mathrm{H}_{18} \mathrm{O}$ & l-Borneol & 154.14 & 56 & $1,467.2$ & $6,134.4$ & $1,465.3$ & 89. \\
\hline $\mathrm{C}_{10} \mathrm{H}_{20} \mathrm{O}$ & Menthol (s) & 156.16 & 58 & $\overrightarrow{1}, 508.8$ & $6,309.8$ & $1,517.4$ & 113. \\
\hline
\end{tabular}




\section{TABLES OF DATA-Continued}

\section{CHO COMPOUNDS-Continued}

13. HYDROAROMATIC AND POLYMETHYLENE SECONDARY ALCOHOLS $Q=26.05 \times N+6.5$

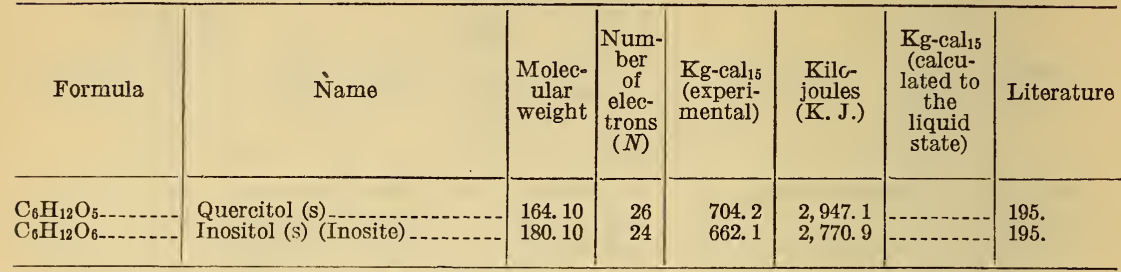

14. TERTIARY ALCOHOLS

$Q=26.05 \times N+3.5$

\begin{tabular}{|c|c|c|c|c|c|c|c|}
\hline $\mathrm{C}_{4} \mathrm{H}_{10} \mathrm{O} \ldots \ldots$ & $\begin{array}{l}\text { Trimethyl carbinol (Tert. } \\
\text { butyl alcohol). }\end{array}$ & 74.08 & 24 & 629.3 & $2,631.7$ & 628.7 & 215 ; cf. 115 . \\
\hline $\mathrm{C}_{5} \mathrm{H}_{12} \mathrm{O}_{\ldots} \ldots \ldots$ & Dimethylethyl carbinol..... & 88.10 & 30 & 784.6 & $3,281.2$ & 785.0 & $215 ;$ cf. 110. \\
\hline $\mathrm{C}_{6} \mathrm{H}_{12} \mathrm{O}_{-}$ & Allyldimethyl carbinol_....- & 100.10 & 34 & $\begin{array}{l}886.5 \\
913.7\end{array}$ & $\begin{array}{l}3,707.3 \\
3,821.1\end{array}$ & 902.2 & $\begin{array}{l}215 . \\
113 .\end{array}$ \\
\hline $\mathrm{C}_{6} \mathrm{H}_{14} \mathrm{O}_{-}$ & Methyldiethyl carbinol_. & 102.11 & 36 & 927.0 & $3,876.7$ & 941.3 & 215. \\
\hline${ }_{7} \mathrm{H}_{14} \mathrm{O}$ & Allylmethylethyl carbinol... & 114. 11 & 40 & $1,050.1$ & $4,391.5$ & $1,058.5$ & 215. \\
\hline $\mathrm{C}_{7} \mathrm{H}_{16} \mathrm{O}_{-}$ & Triethyl carbinol & 116.13 & 42 & $1,080.0$ & $4,516.6$ & $1,097.6$ & 215. \\
\hline $\mathrm{C}_{8} \mathrm{H}_{14} \mathrm{O}$ & Diallylmethyl carbinol .- & 126.11 & 44 & $1,180.7$ & $4,937.7$ & $1,175.7$ & 215 ; cf. 113 \\
\hline TI & Allylmethylpropyl carbinol.- & 128.13 & 46 & $1,201.9$ & $5,026.4$ & $1,214.8$ & 215. \\
\hline $\mathrm{C}_{8} \mathrm{~F}$ & Allyldiethyl carbinol & 128.13 & 46 & $1,207.1$ & $5,048.1$ & $1,214.8$ & 215. \\
\hline $\mathrm{C}_{8} \mathrm{H}_{18}$ & Methyldipropyl carbinol_. & 130. 14 & 48 & $1,232.7$ & $5,155.2$ & $1,253.9$ & 215. \\
\hline & $\begin{array}{l}\text { Allylmethyl- } n \text {-butyl car- } \\
\text { binol. }\end{array}$ & 142.14 & 52 & $1,364.9$ & $5,708.0$ & $1,371.1$ & 215. \\
\hline $\mathrm{C}_{2} \mathrm{H}_{18} \mathrm{O}$ & $\begin{array}{l}\text { Allylmethyl-tert.-butyl car- } \\
\text { binol. }\end{array}$ & 142.14 & 52 & $1,363.0$ & $5,700.0$ & $1,371.1$ & 215. \\
\hline $\mathrm{C}_{9} \mathrm{H}_{20} \mathrm{O}$ & Ethyldipropyl carbinol...... & 144. 16 & 54 & $1,386.5$ & $5,798.3$ & $1,410.2$ & 215. \\
\hline $\mathrm{C}_{1}, \mathrm{H}_{13} \mathrm{O}$ & Diallylpropyl carbinol. & 154. 14 & 56 & $1,472.1$ & $6,156.3$ & $1,488.3$ & 215. \\
\hline $\mathrm{C}_{10} \mathrm{H}_{20} \mathrm{O}$ & Allyldipropyl carbinol. & 156.16 & 58 & & $\begin{array}{l}6,348.3 \\
6,480.4\end{array}$ & $1,527.4$ & $\begin{array}{l}215 . \\
113 .\end{array}$ \\
\hline $\mathrm{C}_{11} \mathrm{H}_{22} \mathrm{O}$ & Allylmethylhexyl carbino & 170. 18 & 64 & $1,666.7$ & $6,970.1$ & $1,683.7$ & 215 . \\
\hline & Triphenyl carbinol (s) & 260.13 & 90 & $2,340.8$ & $9,796.3$ & $2,337.5$ & $182 ;$ cf. 179. \\
\hline & $\begin{array}{l}\text { Diphenylphenylethinyl car- } \\
\text { binol.36 }\end{array}$ & 284.12 & 98 & $2,572.3$ & $10,757.4$ & $2,576.0$ & 95. \\
\hline $\mathrm{C}_{7} \mathrm{H}_{14} \mathrm{O}$ & 1,3-Dimethylcyclopentanol-3 & 114.11 & 40 & $1,034.0$ & $4,324.2$ & $1,045.7$ & 215. \\
\hline $\mathrm{C}_{8} \mathrm{H}_{16} \mathrm{O}$ & 1,2-Dimethylcyclohexanol-2. & 128.13 & 46 & $1,196.5$ & $5,012.1$ & $1,201.8$ & 163. \\
\hline$-\cdots$ & 1,3-Dimethylcyclohexanol-3. & 128.13 & 46 & $1,192.5$ & $4,987.0$ & $1,201.8$ & 215 . \\
\hline$-0-0-1$ & $\begin{array}{l}\text { 1, 3, 5-Trimethylcyclohexene- } \\
\text { 6-ol-5. }\end{array}$ & 140.13 & 50 & $1,294.7$ & $5,414.4$ & $1,312.5$ & 215. \\
\hline $\mathrm{C}_{9} \mathrm{H}_{18} \mathrm{O}$ & $\begin{array}{l}\text { 1-Methyl-3-ethylcyclohexan- } \\
\text { ol-3. }\end{array}$ & 142.14 & 52 & & $5,530.3$ & $1,358.1$ & 215. \\
\hline $\mathrm{C}_{10} \mathrm{H}_{18} \mathrm{O}$ & Terpineol (s) & 154.14 & 56 & $\begin{array}{l}1,469.5 \\
\text { up to } \\
1,480.0\end{array}$ & $\begin{array}{l}6,145.4 \\
6,189.4\end{array}$ & $1,468.8$ & 125. \\
\hline${ }_{10} \mathrm{H}_{22} \mathrm{O}_{3} \ldots \ldots$ & Terpine hydrate (s) - & 190.18 & 56 & $1,451.0$ & $6,068.1$ & & 125. \\
\hline
\end{tabular}

${ }^{36}\left(\mathrm{C}_{6} \mathrm{H}_{5}\right)_{2}:-\mathrm{C}-\mathrm{C} \equiv \mathrm{C}-\mathrm{C}_{6} \mathrm{H}_{5}$.

OेH

15. POLYHYDROXY ALIPHATIC ALCOHOLS

$Q=26.05 \times N+13 \mathrm{j}+6.5 \mathrm{k}+3.5 \mathrm{I}$

\begin{tabular}{|c|c|c|c|c|c|c|c|}
\hline $\mathrm{C}_{2} \mathrm{H}_{6} \mathrm{O}$ & Ethylene glycol ${ }^{37}$ & 62.05 & 10 & 281.9 & $1,179.8$ & 286.5 & 195; cf. 109. \\
\hline $\mathrm{C}_{3} \mathrm{H}$ & Propylene glycol & 76.06 & 16 & 431.0 & $1,802.4$ & 436. 3 & 111. \\
\hline 0 & Isopropylene glycol & 76. 06 & 16 & 436. 1 & $1,823.8$ & 442.8 & 111. \\
\hline $\mathrm{C}_{3}$ & Glycerol....... & 92.06 & 14 & 397.0 & $1,661.5$ & 397.2 & 195; cf. 181, \\
\hline $\mathrm{C}_{4} \mathrm{H}_{10} \mathrm{O}_{4--}$ & Erythritol (s) & 122. 08 & 18 & 504.1 & $2,109.7$ & 507.9 & $\begin{array}{l}\text { 182; cf. 126, } \\
31 \text {. }\end{array}$ \\
\hline $\mathrm{C}_{5} \mathrm{H}_{12} \mathrm{O}_{4}$ & Pentaerythritol (s) & 136: 10 & 24 & 661.2 & $2,767.1$ & 657.7 & 195. \\
\hline $\mathrm{C}_{5} \mathrm{H}_{12} \mathrm{O}_{5} \ldots \ldots$ & Arabitol (s) & 152. 10 & 22 & 611.8 & $2,560.4$ & 618.6 & 195. \\
\hline $\mathrm{C}_{6} \mathrm{H}_{14} \mathrm{O}_{2} \ldots$ & $\begin{array}{l}\text { Pinacol (s) (Tetramethyl- } \\
\text { ethylene glycol). }\end{array}$ & 118.11 & 34 & 897.6 & $3,753.8$ & 898.7 & 115. \\
\hline
\end{tabular}

37 The heat of combustion of diethylene glycol, $\mathrm{HO}-\mathrm{CH}_{2} \cdot \mathrm{CH}_{2} . \mathrm{O}-\mathrm{CH}_{2} \cdot \mathrm{CH}_{2} \mathrm{OH}$ (liq.), is given by Wm. H. Rinkenbach (156) as $566.7 \mathrm{~kg}$.-cal ${ }_{15}$ per mole. No experimental details as to procedure employed are given, 


\section{TABLES OF DATA-Continued}

\section{CHO COMPOUNDS-Continued}

15. POLYHYDROXY ALIPHATIC ALCOHOLS-Continued $Q=26.05 \times N+13 j+6.5 \mathrm{k}+3.51$

\begin{tabular}{|c|c|c|c|c|c|c|c|}
\hline Formula & Name & $\begin{array}{c}\text { Molec- } \\
\text { ular } \\
\text { weight }\end{array}$ & $\begin{array}{l}\text { Num- } \\
\text { ber } \\
\text { of } \\
\text { elec- } \\
\text { trons } \\
(N)\end{array}$ & $\begin{array}{l}\mathrm{Kg} \text {-cal } \\
\text { (experi- } \\
\text { mental) }\end{array}$ & $\begin{array}{l}\text { Kilo- } \\
\text { joules } \\
\text { (K. J.) }\end{array}$ & $\begin{array}{l}\mathrm{Kg} \mathrm{cal}_{15} \\
\text { (calcu- } \\
\text { lated to } \\
\text { the } \\
\text { liquid } \\
\text { state) }\end{array}$ & Literature \\
\hline $\mathrm{C}_{6} \mathrm{H}_{14} \mathrm{O}_{6} \ldots$ & $d$-Mannitol (s) & 182.11 & 26 & 727. 6 & $3,045.0$ & 729.4 & 195 ; cf. 182. \\
\hline $\mathrm{C}_{6} \mathrm{H}_{14} \mathrm{O}_{6--}$ & Dulcitol (s) & 182.11 & 26 & $\begin{array}{l}729.1 \\
723.7\end{array}$ & $\begin{array}{l}3,049.1 \\
3,028.7\end{array}$ & 729.4 & \\
\hline $\mathrm{C}_{7} \mathrm{Fi}_{10} \mathrm{O}_{7--}$ & $\begin{array}{l}\text { Perseitol (Glucoheptol) (s) } \\
\text { (d-mannoheptol). }\end{array}$ & 212.13 & 30 & 835.8 & $3,497.8$ & 840.0 & $195 ;$ cf. 68. \\
\hline $\mathrm{C}_{8} \mathrm{H}_{14} \mathrm{O}_{2-}$ & Tetramethylbutinediol & 142.11 & 42 & $1,142.3$ & $4,780.5$ & $1,134.2$ & 178. \\
\hline $\mathrm{C}_{8} \mathrm{H}_{16} \mathrm{O}_{2}$ & $\begin{array}{l}\text { Tetramethylbutenediol (s) } \\
\text { (Maleinoid). }\end{array}$ & 144. 13 & 44 & $1,172.3$ & $4,906.1$ & $1,166.2$ & 173. \\
\hline $\mathrm{C}_{8} \mathrm{H}_{16} \mathrm{O}_{2}$ & $\begin{array}{l}\text { Tetramethylbutenediol (s) } \\
\text { (Fumaroid). }\end{array}$ & 144. 13 & 44 & $1,175.7$ & $4,920.3$ & $1,166.2$ & 173. \\
\hline $\mathrm{C}_{14} \mathrm{H}_{14} \mathrm{O}$ & Hydrobenzoin (s) 38 & 214. 11 & 66 & $1,723.0$ & $7,210.8$ & $1,725.3$ & 10. \\
\hline $\mathrm{C}_{14} \mathrm{H}_{14} \mathrm{O}_{2}$ & Isohydrobenzoin (s) ${ }^{38}$ & 214.11 & 66 & $1,727.8$ & $7,230.8$ & $1,725.3$ & 10. \\
\hline
\end{tabular}

${ }_{38}$ The form used is not indicated. See, however, Erlenmeyer, jr., Ber., 30, p. 1537; 1897.

16. HYDROAROMATIC AND POLYMETHYLENE GLYCOLS

(All types)

$Q=26.05 \times N+13 \mathrm{j}+6.5 \mathrm{k}+3.5 \mathrm{l}$

\begin{tabular}{|c|c|c|c|c|c|c|c|}
\hline $\mathrm{C}_{5} \mathrm{H}_{10} \mathrm{O}_{2-}$ & C yclopentaned io $1-1,2$ & 102.08 & 26 & 696.1 & $2,913.2$ & 690.3 & 237. \\
\hline $\mathrm{C}_{5} \mathrm{H}_{10} \mathrm{O}_{2}$ & Cyclopentanedio $1-1,2$ & 102.08 & 26 & 694.2 & $2,905.2$ & 690.3 & 237. \\
\hline $\mathrm{C}_{6} \mathrm{H}_{12} \mathrm{O}_{2}$ & Cyclohexanediol-1,2 (cis) & 116.09 & 32 & 841.6 & $3,522.1$ & 846.3 & 237. \\
\hline $\mathrm{C}_{6} \mathrm{H}_{12} \mathrm{O}_{2}$ & Cyclohexanediol-1,2 (trans) & 116. 09 & 32 & 842.7 & $3,526.7$ & 846.3 & 237. \\
\hline $\mathrm{C}_{7} \mathrm{H}_{14} \mathrm{O}_{2}$ & $\begin{array}{l}\text { 1-M et h y l c yclohexane-1,2- } \\
\text { diol (cis). }\end{array}$ & 130. 11 & 38 & 992.6 & $4,154.0$ & 999.9 & 237. \\
\hline $\mathrm{C}_{7} \mathrm{H}_{14} \mathrm{O}_{2}$ & 1-Methylcyclohexane - 1,2 - & 130.11 & 38 & 995.1 & $4,164.5$ & 999.9 & 237. \\
\hline $\mathrm{C}_{9} \mathrm{H}_{10} \mathrm{O}_{2}$ & Hydrindene-1,2-diol (cis).... & 150.08 & 42 & $1,098.5$ & $4,597.2$ & $1,100.1$ & 237. \\
\hline $\mathrm{C}_{9} \mathrm{H}$ & IIydrindene-1,2-diol (trans) & 150.08 & 42 & $1,096.7$ & $4,589.7$ & $1,100.1$ & 237. \\
\hline $\mathrm{C}_{10} \mathrm{H}_{12}$ & $1,2,3,4-$ Tetrahydronaphtha- & 164.09 & 48 & $1,250.7$ & $5,234.2$ & $1,256.4$ & 237. \\
\hline $\mathrm{C}_{10} \mathrm{H}_{12} \mathrm{O}_{2}$ & $\begin{array}{l}\text { 1,2,3,4-Tetrahydronaphtha- } \\
\text { lene-1,2-diol (trans). }\end{array}$ & 164.09 & 48 & $1,249.4$ & $5,228.7$ & $1,256.4$ & $23 \dot{7}$. \\
\hline $\mathrm{C}_{10} \mathrm{H}_{12} \mathrm{O}_{2}$ & 1,2,3,4-Tetrahydronaphtha- & 164.09 & 48 & $1,250.9$ & $5,235.0$ & $1,256.4$ & 237. \\
\hline $\mathrm{C}_{10} \mathrm{H}_{12} \mathrm{O}$ & 1,2,3,4-Tetrahydronaphtha- & 164.09 & 48 & $1,249.4$ & $5,228.7$ & $1,256.4$ & 237. \\
\hline $\mathrm{C}_{12} \mathrm{H}_{16} \mathrm{O}_{2}$ & 1-Phenylcyclohexane-1,2- & 192.12 & 60 & $1,563.1$ & $6,541.6$ & $1,569.5$ & 237. \\
\hline $\mathrm{C}_{12} \mathrm{H}_{16} \mathrm{O}_{2}$ & $\begin{array}{l}\text { 1-Phenylcyclohexano-1,2- } \\
\text { diol (trans). }\end{array}$ & 192.12 & 60 & $1,564.9$ & $6,549.1$ & $1,569.5$ & 237. \\
\hline
\end{tabular}

${ }_{30}$ The heats of combustion of the acetates and benzoates of these compounds are given below (observer 237):

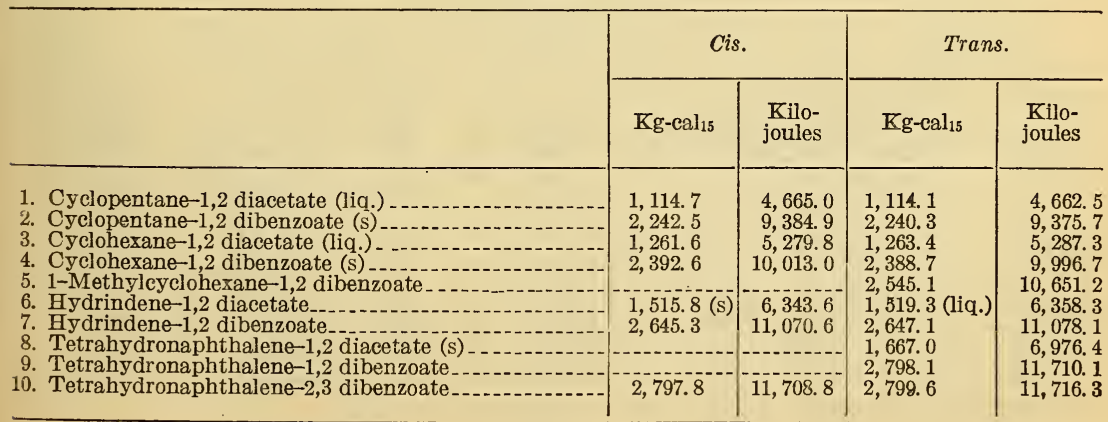




\section{TABLES OF DATA-Continued}

\section{CHO COMPOUNDS-Continued}

17. PHENंOLS

$Q=26.05 \times N+3.5-3.5 \mathrm{a}-6.5 \mathrm{~b}$

\begin{tabular}{|c|c|c|c|c|c|c|c|}
\hline Formula & Name & $\begin{array}{l}\text { Molec- } \\
\text { ular } \\
\text { weight }\end{array}$ & $\begin{array}{c}\text { Num- } \\
\text { ber } \\
\text { of } \\
\text { elec- } \\
\text { trons } \\
(N)\end{array}$ & $\begin{array}{l}\text { Kg-cal } 15 \\
\text { (experi- } \\
\text { mental) }\end{array}$ & $\begin{array}{l}\text { Kilo- } \\
\text { joules } \\
\text { (K. J.) }\end{array}$ & $\begin{array}{l}\mathrm{Kg}-\mathrm{cal}_{15} \\
\text { (calcu- } \\
\text { lated to } \\
\text { the } \\
\text { liquid } \\
\text { state) }\end{array}$ & Literature \\
\hline $\begin{array}{l}\mathrm{C}_{6} \mathrm{H}_{6} \mathrm{O}_{-} \\
\mathrm{C}_{6} \mathrm{H}_{6} \mathrm{O}_{2-} \\
\mathrm{C}_{6} \mathrm{H}_{6} \mathrm{O}_{2}\end{array}$ & $\begin{array}{l}\text { Phenol (s) } \\
\text { Pyrocatechol (s) } \\
\text { Resorcinol (s) }\end{array}$ & $\begin{array}{r}94.05 \\
110.05 \\
110.05\end{array}$ & $\begin{array}{l}28 \\
26 \\
26\end{array}$ & $\begin{array}{l}732.2 \\
684.8 \\
683.0\end{array}$ & $\begin{array}{l}3,064.3 \\
2,865.9 \\
2,858.4\end{array}$ & $\begin{array}{l}732.9 \\
684.3 \\
684.3\end{array}$ & $\begin{array}{l}\text { 195; cf. } 30 . \\
195 . \\
195 .\end{array}$ \\
\hline $\mathrm{C}_{6} \mathrm{H}_{6} \mathrm{O}_{2-}$ & Hydroquinol (s) & 110.05 & 26 & $\left\{\begin{array}{l}682.9 \\
683.7\end{array}\right.$ & $\begin{array}{l}2,857.9 \\
2,859.2\end{array}$ & 684.3 & $\begin{array}{l}\text { 195. } \\
225 ; \text { cf. } 30 \text {, } \\
180 \text {. }\end{array}$ \\
\hline $\begin{array}{l}\mathrm{C}_{6} \mathrm{H}_{6} \mathrm{O}_{3--} \\
\mathrm{C}_{6} \mathrm{H}_{6} \mathrm{O}_{3--}\end{array}$ & $\begin{array}{l}\text { Pyrogallol (s) } \\
\text { Phloroglucinol }(\mathrm{s})_{-}\end{array}$ & $\begin{array}{l}126.05 \\
126.05\end{array}$ & $\begin{array}{l}24 \\
24\end{array}$ & $\begin{array}{r}638.7 \\
40617.3\end{array}$ & $\begin{array}{l}2,672.9 \\
2,583.4\end{array}$ & $\begin{array}{l}635.7 \\
635.7\end{array}$ & 195 ; cf. 30 . \\
\hline $\mathrm{C}_{7} \mathrm{H}_{8} \mathrm{O}_{\ldots} .$. & $\left\{\begin{array}{l}0-\text { Cresol } \\
0-\text { Cresol }(\mathrm{s})\end{array}\right.$ & $\begin{array}{l}108.06 \\
108.06\end{array}$ & $\begin{array}{l}34 \\
34\end{array}$ & $\begin{array}{r}41882.6 \\
879.5\end{array}$ & $\begin{array}{l}3,693.7 \\
3,680.7\end{array}$ & 885.7 & 201. \\
\hline $\mathrm{C}_{7} \mathrm{H}_{8} \mathrm{O}$. & m-Cresol.... & 108.06 & 34 & 880.5 & $\begin{array}{r}3,684.9 \\
423,746.0\end{array}$ & 885.7 & 201. \\
\hline $\begin{array}{l}\mathrm{C}_{7} \mathrm{H}_{8} \mathrm{O}_{2} \\
\mathrm{C}_{7} \mathrm{H}_{8} \mathrm{O}_{2-}\end{array}$ & $\left\{\begin{array}{l}p \text {-Cresol } 43-\ldots \\
p \text {-Cresol }(\mathrm{s})-\ldots\end{array}\right.$ & $\begin{array}{l}108.06 \\
108.06 \\
124.06\end{array}$ & $\begin{array}{l}34 \\
34 \\
32\end{array}$ & $\begin{array}{l}882.5 \\
880.0 \\
824.3\end{array}$ & $\begin{array}{l}3,693.3 \\
3,682.8 \\
3,449.7\end{array}$ & 885.7 & $\begin{array}{l}211 . \\
211 . \\
201 .\end{array}$ \\
\hline $\begin{array}{l}\mathrm{C}_{7} \mathrm{H}_{8} \mathrm{O}_{2-}- \\
\mathrm{C}_{7} \mathrm{H}_{8} \mathrm{O}_{2-} \\
\mathrm{C}_{8} \mathrm{H}_{10} \mathrm{O}_{-} \\
\mathrm{C}_{8} \mathrm{H}_{10} \mathrm{O}_{-} \\
\mathrm{C}_{8} \mathrm{H}_{10} \mathrm{O}_{--}\end{array}$ & $\begin{array}{l}\text { Saligenin (s) } \\
\text { Toluhydroquinol (s) } \\
o-\text { Xylenol (s) } \\
m \text {-Xylenol (s) } \\
p-\text { Xylenol (s) }\end{array}$ & $\begin{array}{l}124.06 \\
124.06 \\
122.08 \\
122.08 \\
122.08\end{array}$ & $\begin{array}{l}32 \\
32 \\
40 \\
40 \\
40\end{array}$ & $\begin{array}{r}845.4 \\
835.1 \\
1,035.1 \\
1,037.4 \\
1,035.3\end{array}$ & $\begin{array}{l}3,535.5 \\
3,494.9 \\
4,331.9 \\
4,341.5 \\
4,332.7\end{array}$ & $\begin{array}{r}846.6 \\
837.1 \\
1,038.5 \\
1,038.5 \\
1,038.5\end{array}$ & $\begin{array}{l}44 . \\
225 . \\
201 . \\
201 . \\
201 .\end{array}$ \\
\hline $\begin{array}{l}\mathrm{C}_{9} \mathrm{H}_{12} \mathrm{O}_{--} \\
\mathrm{C}_{10} \mathrm{H}_{8} \mathrm{O}_{-} \\
\mathrm{C}_{10} \mathrm{H}_{8} \mathrm{O}_{-} \\
\mathrm{C}_{10} \mathrm{H}_{14} \mathrm{O}_{--}\end{array}$ & $\begin{array}{l}\text { Pseudocumenol (s) } \\
\alpha \text {-Naphthol (s) } \\
\beta \text {-Naphthol (s) } \\
\text { Thymol } \\
\text { Thyymol (s) }\end{array}$ & $\begin{array}{l}136.10 \\
144.06 \\
144.06 \\
150.11\end{array}$ & $\begin{array}{l}46 \\
46 \\
46 \\
52\end{array}$ & $\begin{array}{l}1,191.2 \\
1,185.4 \\
1,187.2 \\
1,353.4 \\
1,349.7\end{array}$ & $\begin{array}{l}4,985.2 \\
4,960.9 \\
4,968.4 \\
5,663.9 \\
5,648.5\end{array}$ & $\begin{array}{l}1,191.3 \\
1,188.8 \\
1,188.8 \\
1,351.1\end{array}$ & $\begin{array}{l}201 . \\
225 . \\
225 . \\
201 . \\
201 .\end{array}$ \\
\hline $\begin{array}{l}\mathrm{C}_{10} \mathrm{H}_{14} \mathrm{O} \\
\mathrm{C}_{10} \mathrm{H}_{14} \mathrm{O}_{2-} \\
\mathrm{C}_{14} \mathrm{H}_{10} \mathrm{O}_{2}\end{array}$ & $\begin{array}{l}\text { Carvacrol } \\
\text { Thymohydroquinol (s) } \\
\text { Phenanthrahydroquinol }\end{array}$ & $\begin{array}{l}150.11 \\
166.11 \\
210.08\end{array}$ & $\begin{array}{l}52 \\
50 \\
62\end{array}$ & $\begin{array}{l}1,354.5 \\
1,307.1 \\
1,600.1\end{array}$ & $\begin{array}{l}5,668.6 \\
5,470.2 \\
6,696.4\end{array}$ & $\begin{array}{l}1,351.1 \\
1,302.5 \\
1,602.6\end{array}$ & $\begin{array}{l}201 . \\
225 . \\
225 .\end{array}$ \\
\hline $\mathrm{C}_{21} \mathrm{H}_{16} \mathrm{O}_{2}$ & $\begin{array}{l}\beta \text {-Dioxydinaph thylmethane } \\
(\mathrm{s}) \text {. }\end{array}$ & 300.13 & 96 & $2,475.8$ & $10,353.8$ & $2,474.8$ & 60. \\
\hline
\end{tabular}

40 The determinations by (202) were carried out in oxygen at ordinary pressure and differ greatly from later determinations by (195). Thus, (202) give for pyrogallol $616.3 \mathrm{~kg}$-cal ${ }_{15}$, but (195) give $639.0 \mathrm{~kg}$-cal 15 per mole. The above volue for phloroglucinol is undoubtedly very low. It should be approximately the same as that for pyrogallol.

41 It is quite possible that the same objection and the inherent error in the determinations occur also in the values of (201) as occur in (202). See footnote 40.

${ }_{42}$ This value is undoubtedly too high.

${ }_{43}$ M. F. Barker, J. Phys. Chem. 29, p. 1350, 1925, reports the following values for the three cresols at constant pressure: $\omega-(\mathrm{s}), 883.7 ; p-(\mathrm{s}), 885.0 ; m$-(liq.), 883.0. However, no experimental details are available except the final values.

\section{ALIPHATIC ETHERS 44}

$Q=26.05 \times N+19.5$

\begin{tabular}{|c|c|c|c|c|c|c|c|}
\hline $\mathrm{C}_{2} \mathrm{H}_{6} \mathrm{O}_{\ldots}$. & Dimethyl ether (g). & 46 & 12 & 347.6 & $1,453.7$ & & 223 ; cf. 10. \\
\hline $\mathrm{C}_{3} \mathrm{H}_{8} \mathrm{O}_{\ldots} \ldots \ldots$ & Methylethyl ether $(\mathrm{v})$ & 60 & 18 & 503.4 & $2,105.2$ & $0-2-0-2$ & 223 . \\
\hline $\mathrm{C}_{4} \mathrm{H}_{6} \mathrm{O}$ & Methylpropargyl ether $(\nabla)$ & 70.00 & 20 & 600.8 & $2,512.6$ & (2) & 223. \\
\hline $\mathrm{C}_{4} \mathrm{H}_{8} \mathrm{O}$ & Methylallyl ether (v) & 72. 00 & 22 & 623.9 & $2,609.2$ & & 223. \\
\hline $\mathrm{C}_{4} \mathrm{H}_{10} \mathrm{O}$ & $\left\{\begin{array}{l}\text { Diethyl ether } \\
\text { Diethyl ether }(\nabla)\end{array}\right.$ & 74. 08 & 24 & $\begin{array}{l}651.7 \\
660.3\end{array}$ & $\begin{array}{l}2,727.4 \\
2,763.4\end{array}$ & & $\begin{array}{l}204 . \\
204 .\end{array}$ \\
\hline $\mathrm{C}_{6} \mathrm{H}_{10} \mathrm{O}$ & Diallyl ether $(v)$. & 98 & 32 & 906.6 & $3,791.4$ & & 223. \\
\hline $\mathrm{C}_{6} \mathrm{H}_{10} \mathrm{O}_{2}$ & $\begin{array}{l}\text { Acetylacetone-O-methyl } \\
\text { ether. }\end{array}$ & 114.08 & 30 & 817.8 & $3,422.5$ & 82 & 166. \\
\hline $\mathrm{C}_{6} \mathrm{H}_{14} \mathrm{C}$ & Glycoldiethyl ether & 118. 11 & 34 & 924.6 & $3,869.5$ & 924.7 & 172. \\
\hline $\mathrm{C}_{7} \mathrm{H}_{12} \mathrm{O}_{2} \ldots$ & Acetylacetone-O-ethyl ether & 128.10 & 36 & 968.0 & $4,051.1$ & 976.8 & 166. \\
\hline $\mathrm{C}_{10} \mathrm{H}_{22} \mathrm{O} \ldots$ & Diamyl ether....................... & 158.17 & 60 & $1,609.3$ & $6,730.1$ & $1,582.0$ & 65. \\
\hline
\end{tabular}

44 For heat of combustion of diethylene glycol, see footnote (37), p. 380. 


\section{TABLES OF DATA-Continued}

\section{CHO COMPOUNDS-Continued}

\section{ALIPHATIC ACETALS 45}

$\mathrm{Q}=26.05 \times \mathrm{N}+19.5 \times 0$

\begin{tabular}{|c|c|c|c|c|c|c|c|}
\hline Formula & Name & $\begin{array}{l}\text { Molec- } \\
\text { ular } \\
\text { weight }\end{array}$ & $\begin{array}{c}\text { Num- } \\
\text { ber } \\
\text { of } \\
\text { elec- } \\
\text { trons } \\
(N)\end{array}$ & $\begin{array}{l}\mathrm{Kg} \text {-cal }{ }_{15} \\
\text { (experi- } \\
\text { mental) }\end{array}$ & $\begin{array}{c}\text { Kilo- } \\
\text { joules } \\
\text { (K. J.) }\end{array}$ & $\begin{array}{l}\mathrm{Kg}-\mathrm{cal}_{15} \\
\text { (calcu- } \\
\text { lated to } \\
\text { the } \\
\text { liquid } \\
\text { state) }\end{array}$ & Literature \\
\hline $\mathrm{C}_{3} \mathrm{H}_{6} \mathrm{O}_{2--}$ & $\begin{array}{l}\text { Glycolmethylal (Methylene- } \\
\text { ethylene dioxide). }\end{array}$ & 74.05 & 14 & 409.9 & $1,715.43$ & 403.7 & 59. \\
\hline $\mathrm{C}_{3} \mathrm{H}_{8} \mathrm{O}_{2}-$ & Methylal & 76.06 & 16 & 462.8 & $1,936.8$ & 455.8 & 26. \\
\hline $\mathrm{C}_{4} \mathrm{H}_{6} \mathrm{O}_{2-}$ & $\begin{array}{l}\text { Glycolacetal (Ethylidene- } \\
\text { acetal of glycol). }\end{array}$ & 88.06 & 20 & 559.2 & $2,340.3$ & 560.1 & 59. \\
\hline $\mathrm{C}_{4} \mathrm{H}_{10} \mathrm{O}_{2}$ & Dimethylacetal...... & 90.09 & 22 & 620.0 & $2,594.7$ & 612.1 & 58. \\
\hline $\mathrm{C}_{4} \mathrm{H}_{10} \mathrm{O}_{3}$ & Methyl orthoformate & 106. 0 & 20 & 46596.8 & $2,495.8$ & 579.5 & 223. \\
\hline $\mathrm{C}_{5} \mathrm{H}_{12} \mathrm{O}_{2-}$ & Diethylmethylal... & 104.10 & 28 & $\begin{array}{r}774.5 \\
4733.8\end{array}$ & $\begin{array}{l}3,241.3 \\
3,238.4\end{array}$ & 768.2 & $\begin{array}{l}58 . \\
103 .\end{array}$ \\
\hline $\mathrm{C}_{6} \mathrm{H}_{14} \mathrm{O}_{2}$ & Diethylacetal.- & 118.11 & 34 & $\begin{array}{r}923.0 \\
931.2 \\
919.5\end{array}$ & $\begin{array}{l}3,862.8 \\
3,897.1 \\
3,848.1\end{array}$ & 924.7 & $\begin{array}{l}157 . \\
58 . \\
117 .\end{array}$ \\
\hline $\mathrm{C}_{6} \mathrm{H}_{10} \mathrm{O}_{4-}$ & Erythritemethylal (s). & 146.08 & 26 & 745.0 & $3,117.8$ & & 59. \\
\hline $\mathrm{C}_{7} \mathrm{H}_{16} \mathrm{O}_{2-}$ & Dipropylmethylal & 132.13 & 40 & $1,085.9$ & $4,544.5$ & $1,081.1$ & 58. \\
\hline $\mathrm{C}_{7} \mathrm{H}_{14} \mathrm{O}_{6}$ & $\alpha$-Methylglycoside (s) & 194.11 & 30 & 842.5 & $3,529.2$ & $--2-1-2=-1$ & 241. \\
\hline $\mathrm{C}_{8} \mathrm{H}_{14} \mathrm{O}_{4}$ & Erythritediacetal (s) & 174.11 & 38 & $1,049.5$ & $4,392.2$ & $1,067.9$ & 59. \\
\hline $\mathrm{C}_{9} \mathrm{H}_{20}$ & Diisobutylmethylal._._....-. & 160.16 & 52 & $1,395.3$ & $5,839.3$ & $1,393.6$ & 58. \\
\hline $\mathrm{C}_{9} \mathrm{H}_{14} \mathrm{O}_{6}$ & Mannitetrimethylal (s). & 218.11 & 38 & $1,084.1$ & $4,536.9$ & $1,074.4$ & 59. \\
\hline $\mathrm{C}_{11} \mathrm{H}_{24} \mathrm{O}_{2 \ldots} \ldots \ldots$ & Diisoamylmethylal_.- & 188. 19 & 64 & $1,708.7$ & $7,150.9$ & $1,706.2$ & 58. \\
\hline $\mathrm{C}_{12} \mathrm{H}_{20} \mathrm{O}_{6} \ldots \ldots$ & Mannitetriacetal (s) & 260.16 & 56 & $1,539.0$ & $6,440.7$ & & 59. \\
\hline $\mathrm{C}_{12} \mathrm{H}_{22} \mathrm{O}_{2 \ldots} \ldots$ & Amylpropiolic acetal & 198. 18 & 66 & $1,790.7$ & $7,494.1$ & $1,791.4$ & 138. \\
\hline $\mathrm{C}_{12} \mathrm{H}_{22} \mathrm{O}$ & $\begin{array}{l}\text { Diacetal of acetylenedialde- } \\
\text { hyde (s). }\end{array}$ & 230.17 & $62^{\circ}$ & $1,724.9$ & $7,218.7$ & $1,726.4$ & 138. \\
\hline $\mathrm{C}_{13} \mathrm{H}_{24} \mathrm{O}_{2-}$ & Hexylpropiolic acetal & 212.19 & 72 & $1,946.4$ & $8,145.7$ & $1,947.7$ & 138. \\
\hline
\end{tabular}

${ }_{45}$ The values for acetals given by (58 and 59) are about 0.5 per cent to 1.0 per cent too high.

46 Individual determinations do not agree better than 0.5 per cent.

${ }_{47}$ The values of (103) are about 0.6 per cent too high. Better value, $769.2 \mathrm{~kg}$-cal 15 .

${ }_{48}\left(\mathrm{C}_{2} \mathrm{H}_{5} \mathrm{O}\right)_{2}: \mathrm{H}-\mathrm{C}-\mathrm{C} \equiv \mathrm{C}-\mathrm{H}$ : $\left(\mathrm{OC}_{2} \mathrm{H}_{5}\right)_{2}$.

\section{AROMATIC ETHERS}

$Q=26.05 \times N+19.5$

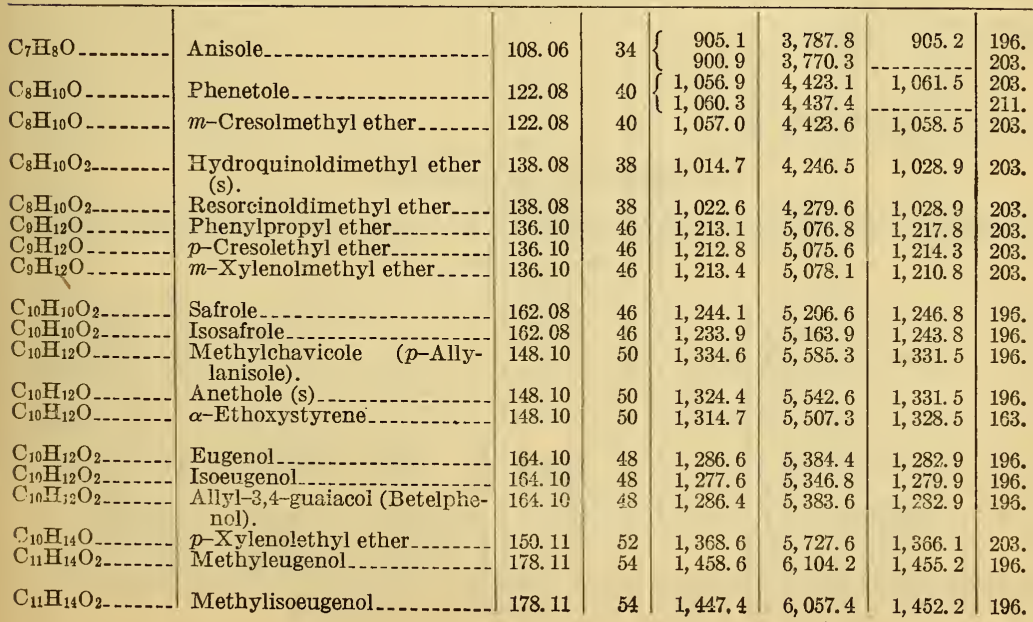




\section{TABLES OF DATA-Continued \\ 2. CHO COMPOUNDS-Continued}

20. AROMATIC ETHERS-Continued

$Q=20.05 \times N+19.5$

\begin{tabular}{|c|c|c|c|c|c|c|c|}
\hline Formula & Name & $\begin{array}{c}\text { Molec- } \\
\text { ular } \\
\text { weight }\end{array}$ & $\begin{array}{l}\text { Num- } \\
\text { ber } \\
\text { of } \\
\text { elec- } \\
\text { trons } \\
(N)\end{array}$ & $\begin{array}{l}\mathrm{Kg} \text { cal }_{15} \\
\text { (experi- } \\
\text { mental) }\end{array}$ & $\begin{array}{c}\text { Kilo- } \\
\text { joules } \\
\text { (K. J.) }\end{array}$ & $\begin{array}{l}\mathrm{Kg}-\mathrm{cal}_{15} \\
\text { (calcu- } \\
\text { lated to } \\
\text { the } \\
\text { liquid } \\
\text { state) }\end{array}$ & Literature \\
\hline $\mathrm{C}_{11} \mathrm{H}_{16} \mathrm{O}$ & Thymolmethyl ether & 164.13 & 58 & $1,524.3$ & $6,379.2$ & $1,523.4$ & 203. \\
\hline $\mathrm{C}_{12} \mathrm{H}_{14} \mathrm{O}_{4}$ & Apiole (s) & 222.11 & 54 & $1,498.8$ & $6,272.5$ & $\overrightarrow{1}, 494.2$ & 196. \\
\hline $\mathrm{C}_{12} \mathrm{H}_{14} \mathrm{O}_{4}$ & Isoapiole (s) & 222.11 & 54 & $1,488.2$ & $6,228.1$ & $1,491.2$ & 196. \\
\hline $\mathrm{C}_{12} \mathrm{H}_{16} \mathrm{O}_{2-}$ & Ethylisoeugenol (s) & 192.13 & 60 & $1,602.3$ & $6,705.6$ & $1,608.5$ & 196. \\
\hline $\mathrm{C}_{12} \mathrm{H}_{16} \mathrm{O}_{3-}$ & $\begin{array}{l}\text { Asarone }(\mathrm{s})^{49} \text { (Propenyl-2,4,5- } \\
\text { trimethoxybenzene). }\end{array}$ & 208. 13 & 58 & $1,576.2$ & $6,596.4$ & $1,575.9$ & 196. \\
\hline $\mathrm{C}_{12} \mathrm{H}_{18} \mathrm{O}_{--}$ & Thymolethyl ether & 178.14 & 64 & $1,679.9$ & $7,030.4$ & $1,679.7$ & 203. \\
\hline $\mathrm{C}_{16} \mathrm{H}_{16} \mathrm{O}_{2}$ & Di-p-methoxystilbene (s) ...- & 240.14 & 76 & $2,016.6$ & $8,433.4$ & $2,018.8$ & 104. \\
\hline $\mathrm{C}_{21} \mathrm{H}_{16} \mathrm{O}_{2} \ldots$ & $\beta$-Naphtholformal (s) & 300.13 & 96 & $2,500.5$ & $10,457.1$ & $2,513.8$ & 60. \\
\hline $\mathrm{C}_{22} \mathrm{H}_{18} \mathrm{O}_{--}$ & $\begin{array}{l}\text { Methyl ether of diphenyl- } \\
\text { phenylethinyl carbinol.50 }\end{array}$ & 298.14 & 104 & $2,739.4$ & $11,456.2$ & $2,748.3$ & 95. \\
\hline $\mathrm{C}_{23} \mathrm{H}_{20} \mathrm{O}$ & $\begin{array}{l}\text { Ethyl ether of diphenyl- } \\
\text { phenylethinyl carbinol. }\end{array}$ & 312.16 & 110 & $2,891.4$ & 12.091 .8 & $2,904.6$ & 95. \\
\hline $\mathrm{C}_{24} \mathrm{H}_{22} \mathrm{O}$ & $\begin{array}{l}\text { Propyl ether of diphenyl- } \\
\text { phenylethinyl carbinol. }\end{array}$ & 326.17 & 116 & $3,047.5$ & $12,744.7$ & $3,060.9$ & 95. \\
\hline
\end{tabular}

$49\left(\mathrm{CH}_{3} \mathrm{O}\right)_{3}(2,4,5)\left(\mathrm{C}_{6} \mathrm{H}_{2}\right)\left(\mathrm{CH}=\mathrm{CH} \cdot \mathrm{CH}_{3}\right){ }^{(1)}$.

${ }^{80}\left(\mathrm{C}_{6} \mathrm{H}_{5}\right)_{2} . \mathrm{C}\left(\mathrm{OCH}_{3}\right) \mathrm{C} \equiv \mathrm{C}_{2} \mathrm{C}_{6} \mathrm{H}_{5}$.

\section{ETHYLENE OXIDES}

( $\alpha$-Oxides)

\begin{tabular}{|c|c|c|c|c|c|c|c|}
\hline & SEthylene oxide $(\nabla)$ & 44.03 & 10 & 308.4 & $1,290.7$ & & 16; cf. 223 . \\
\hline $\mathrm{C}_{2} \mathrm{H}_{4} \mathrm{O}_{-}$ & Ethylene oxide & $-\ldots$ & & 302.1 & $1,264.3$ & $\cdots$ & 16. \\
\hline $\mathrm{C}_{3} \mathrm{H}_{6} \mathrm{O} \ldots$ & a-Propylene oxide 51 & 58.05 & 16 & 451.1 & $1,887.9$ & & 245 . \\
\hline $\mathrm{C}_{5} \mathrm{H}_{10} \mathrm{O}_{-} \ldots$ & $\begin{array}{l}\beta \text {-Methyl- } \beta, \gamma \text {-butene oxide } \\
\text { ( } \alpha \text {-Dimethyltrimethylene } \\
\text { oxide). } 52\end{array}$ & 86. 08 & 28 & 749.8 & $3,137.9$ & $-\ldots-1$ & 245. \\
\hline $\mathrm{C}_{6} \mathrm{H}_{12} \mathrm{O}_{\ldots} \ldots$ & $\gamma, \delta$-Hexylene oxide ${ }^{53} \ldots$ & 100.10 & 34 & 913.9 & $3,824.7$ & & 245. \\
\hline $\mathrm{C}_{9} \mathrm{H}_{8} \mathrm{O} \ldots$ & Indene oxide . . . & 132.06 & 42 & $1,116.8$ & $4,673.8$ & -- & 236. \\
\hline
\end{tabular}

${ }_{0} \mathrm{CH}_{2} \stackrel{\mathrm{H}}{\mathrm{C}}-\mathrm{CH}_{3}$.

$52\left(\mathrm{CH}_{3}\right) 2 \underbrace{\mathrm{C}}_{\mathrm{O}}-\mathrm{C}-\mathrm{CH}_{3}$.

$53 \mathrm{CH}_{3} \cdot \mathrm{CH}_{2} \cdot \underbrace{\mathrm{H}}_{\mathrm{O}}-\stackrel{\mathrm{H}}{\mathrm{C}}-\mathrm{CH}_{2} \cdot \mathrm{CH}_{3}$.

\section{ALIPHATIC ALDEHYDES}

[Also those aromatic aldehydes in which the aldehyde group is not attached to the aromatic nucleus] $Q=26.05 \times N+13.0$

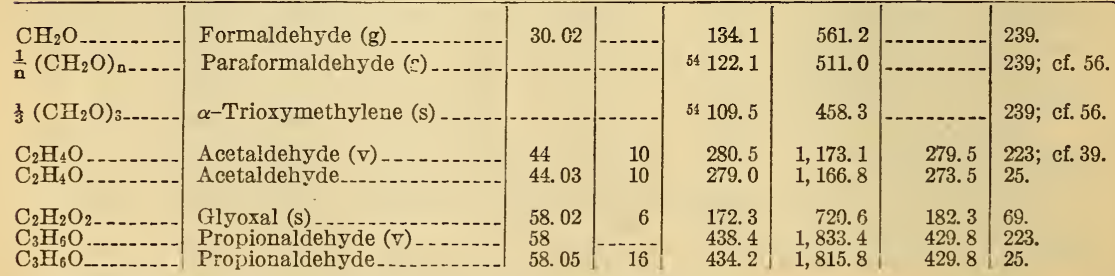

${ }^{54}$ No great reliance can be put upon the above values until the exact details of the determinations becoine available, The information in the article is extremely meager and insufficient. 


\section{TABLES OF DATA-Continued}

\section{CHO COMPOUNDS-Continued}

22. ALIPHATIC ALDEHYDES-Continued

[Also those aromatic aldehydes in which the aldehyde group is not attached to the aromatic nucleus] $Q=26.05 \times N+13.0$

\begin{tabular}{|c|c|c|c|c|c|c|c|}
\hline Formula & Name & $\begin{array}{c}\text { Molec- } \\
\text { ular } \\
\text { weight }\end{array}$ & $\begin{array}{c}\text { Num- } \\
\text { ber } \\
\text { of } \\
\text { elec- } \\
\text { trons } \\
(N)\end{array}$ & $\begin{array}{l}\mathrm{Kg} \text {-cal } \\
\text { (experi- } \\
\text { mental) }\end{array}$ & $\begin{array}{c}\text { Kilo- } \\
\text { joules } \\
\text { (K.J.) }\end{array}$ & $\begin{array}{l}\mathrm{Kg}-\mathrm{cal}_{15} \\
\text { (calcu- } \\
\text { lated to } \\
\text { the } \\
\text { liquid } \\
\text { state) }\end{array}$ & Literature \\
\hline $\mathrm{O}_{3} \mathrm{H}_{4} \mathrm{O}$ & Acrolein. - & 56.03 & 14 & $\left\{\begin{array}{l}389.6 \\
393.4\end{array}\right.$ & $\begin{array}{l}1,629.3 \\
1,646.4\end{array}$ & 390.7 & $\begin{array}{l}141 . \\
238 .\end{array}$ \\
\hline$\left(\mathrm{C}_{3} \mathrm{H}_{4} \mathrm{O}\right)_{3}$ & Metacrolein (s) & 168.09 & & $1,168.8$ & $4,891.4$ & & 238. \\
\hline $\mathrm{C}_{4} \mathrm{H}_{6} \mathrm{O}$ & Crotonaldehyde & 70.05 & 20 & 542.1 & $2,267.1$ & 547.0 & 117. \\
\hline $\mathrm{C}_{4} \mathrm{H}_{8} \mathrm{O}$ & Isobutyraldehyde $(\mathrm{v})$ & & 22 & 596.8 & $2,495.8$ & 586.1 & 223. \\
\hline $\mathrm{C}_{4} \mathrm{H}$ & $\begin{array}{l}\beta \text {-Hydroxybutyraldehyde } \\
\text { (Aldol) }\end{array}$ & 88.06 & 20 & 546.6 & $2,285.9$ & 540.5 & 118. \\
\hline $\mathrm{C}_{5} \mathrm{H}_{10} \mathrm{O}$ & Valeraldehyde & 86.08 & 28 & 742.0 & $3,103.0$ & 742.4 & 113. \\
\hline $\begin{array}{l}\mathrm{C}_{5} \mathrm{H}_{4} \mathrm{O}_{2}- \\
\mathrm{C}_{6} \mathrm{H}_{12} \mathrm{O}_{3}\end{array}$ & $\begin{array}{l}\text { Furol (Furfuraldehyde) } \\
\text { Paraldehyde. }\end{array}$ & $\begin{array}{r}96.03 \\
132.10\end{array}$ & 20 & $\begin{array}{l}559.5 \\
812.8\end{array}$ & $\begin{array}{l}2,339.8 \\
3,399.1\end{array}$ & & $\begin{array}{l}44 . \\
118 .\end{array}$ \\
\hline$\left(\mathrm{C}_{2} \mathrm{H}_{4} \mathrm{O}\right)_{3}$ & $\left\{\begin{array}{l}\text { Metaldehyde }(\mathrm{s}) \\
\text { Metaldehyde }\end{array}\right.$ & 132.10 & & $\begin{array}{l}805.6 \\
814.0\end{array}$ & $\begin{array}{l}3,371.4 \\
3,406.6\end{array}$ & & $\begin{array}{l}126 . \\
126 .\end{array}$ \\
\hline $\mathrm{C}_{7} \mathrm{H}_{14} \mathrm{O}$. & n-Heptaldehyde (Oenanthol) & 114. i1 & 40 & $1,062.4$ & $4,442.9$ & $1,055.0$ & 110. \\
\hline $\mathrm{C}_{9} \mathrm{H}_{6} \mathrm{O}_{-}$ & Phenylpropiolic aldehyde & 130.05 & 40 & $1,081.0$ & $4,520.7$ & $1,081.6$ & 138. \\
\hline $\mathrm{C}_{9} \mathrm{H}_{8} \mathrm{O} \ldots$ & Cinnamic aldehyde. & 132.06 & 42 & $1,112.3$ & $4,655.0$ & $1,113.6$ & 183. \\
\hline $\mathrm{C}_{10} \mathrm{H}_{16} \mathrm{O}_{\ldots} \ldots$ & Citral (Geranial) & 152. 13 & 54 & $1,437.0$ & $6,019.6$ & $1,445.7$ & 174. \\
\hline
\end{tabular}

23. AROMATIC ALDEHYDES

$Q=26.05 \times N+13-3.5 \mathrm{a}+3.5 \mathrm{~m}$

\begin{tabular}{|c|c|c|c|c|c|c|c|}
\hline $\mathrm{C}_{7} \mathrm{H}_{0} \mathrm{O}$ & Benzaldehyde.......... & 106.05 & 32 & 841.3 & $3,520.8$ & 843.1 & 205. \\
\hline $\mathrm{C}_{7} \mathrm{H}_{6} \mathrm{O}_{2}$ & $\begin{array}{l}\text { o-Hydroxybe nzalde hy de } \\
\text { (Salicylaldehyde). }\end{array}$ & 122.05 & 30 & 796.0 & $3,328.9$ & 794.5 & 62. \\
\hline $\mathrm{C}_{7} \mathrm{H}_{6} \mathrm{O}_{2--}$ & $p$-Hydroxybenzaldehyde (s) & 122.05 & 30 & 792.7 & $3,315.0$ & 794.5 & 62. \\
\hline $\mathrm{C}_{7} \mathrm{H}_{6} \mathrm{O}_{2} \ldots$ & $m$-Hydroxybenzaldehyde (s) & 122.05 & 30 & 788.7 & $3,303.9$ & 794.5 & 163. \\
\hline $\mathrm{C}_{8} \mathrm{H}_{8} \mathrm{O}_{3-}$ & $\begin{array}{l}\text { Vanillin (s) (3-Methoxy-4- } \\
\text { hydroxybenzaldehyde). }\end{array}$ & 152.06 & 34 & 914.1 & $3,825.5$ & 918.2 & 183. \\
\hline $\mathrm{C}_{8} \mathrm{H}_{6} \mathrm{O}_{3}$ & Piperonal (s) (Methylenepro- & 150.05 & 32 & 870.1 & $3,641.4$ & 882.1 & 183. \\
\hline $\mathrm{C}_{14} \mathrm{H}_{10} \mathrm{O}_{3}$ & Disalicylic aldehyde (s) & 226.08 & & $1,589.0$ & $\begin{array}{l}3,647.4 \\
6,645.2\end{array}$ & $\mid--$ & $\begin{array}{l}163 . \\
157 .\end{array}$ \\
\hline
\end{tabular}

24. ALIPHATIC KETONES

[Also those ketones in which the ketone group is not attached to the aromatic nucleus] $Q=26.05 \times N+6.5$

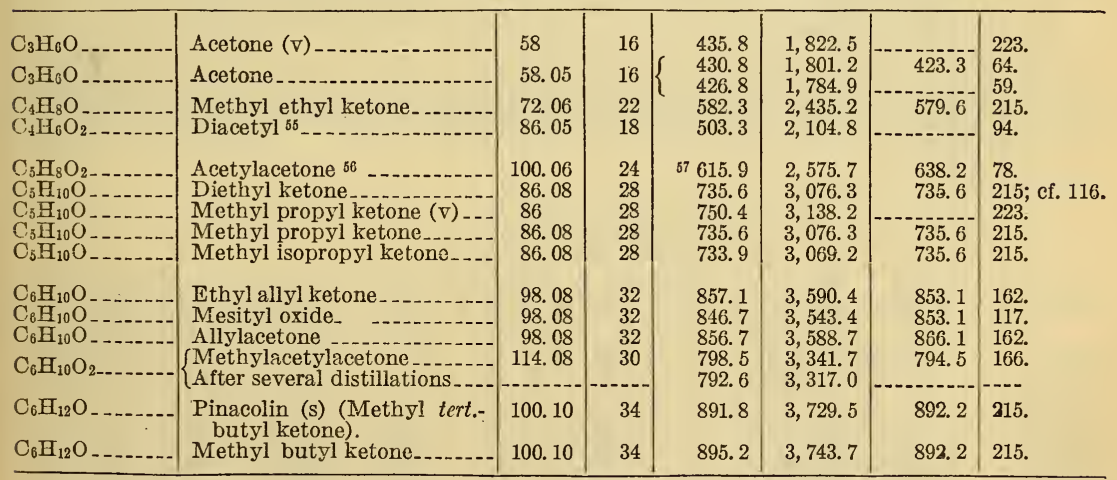

${ }^{65}$ The author (94) gives only the final value for this compound, and no other information. The values of this observer are about 0.5 per cent too low.

${ }_{36}$ See also p. 382 for $O$-methyl and ethyl ethers.

57 Evidently this value by (78) is too low. Compare the rather good agreement in the case of the methylacetylacetone. 


\section{TABLES OF DATA-Continued}

\section{CHO COMPOUNDS-Continued}

24. ALIPHATIC KETONES-Continued

[Also those ketones in which the ketone group is not attached to the aromatic nucleus] $Q=26.05 \times N+6.5$

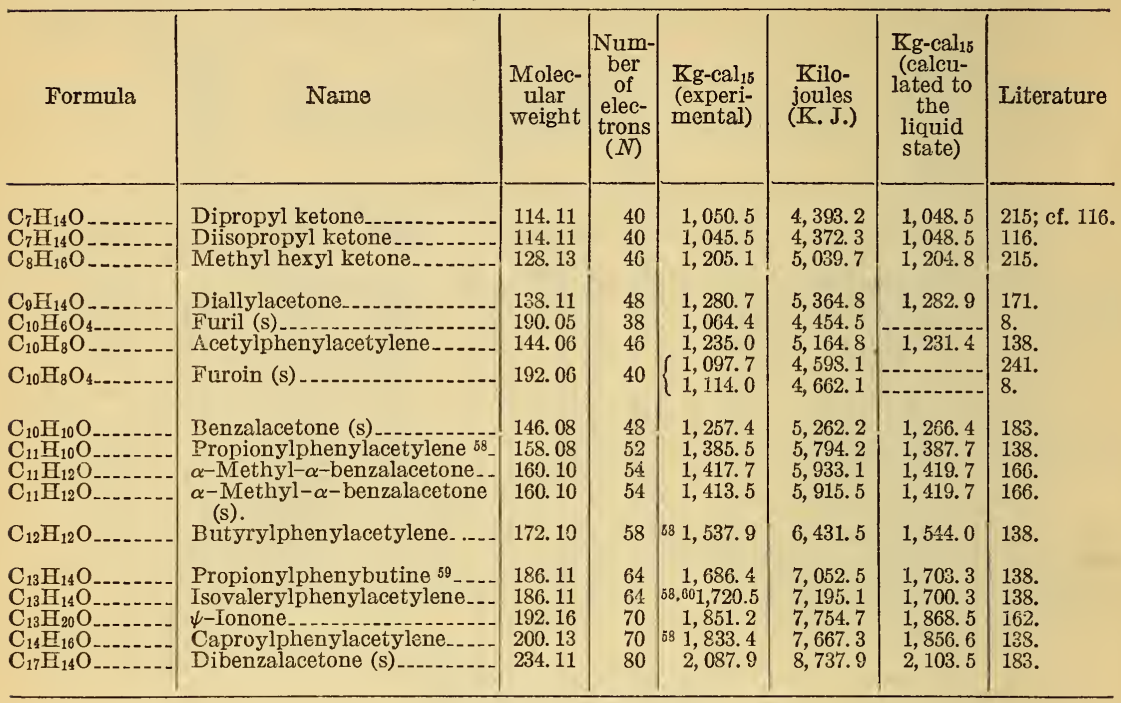

${ }_{58}$ The experimental values of the compounds marked show such wide variations for members of an homologous series that undoubtedly it is the fault of the experimenter. The difflculties of obtaining these substances in pure condition may have had something to do with it. Consult original paper.

${ }_{89} \mathrm{C}_{6} \mathrm{H}_{5} . \mathrm{CH}_{2} \mathrm{CH}_{2}$. $\mathrm{C} \equiv \mathrm{C}-\mathrm{COC}_{2} \mathrm{H}_{5}$.

60 The author (138) claims that this compound behaves abnormally in many respects.

25. AROMATIC KETONES

$Q=26.05 \times N+6.5-3.5 \mathrm{a}-6.5 \mathrm{~b}$

${ }_{01}$ Compare W. A. Roth and R. Lasse, Z. Electrochem., 30, p. 607; 1924. These authors, using a microcombustion apparatus, obtained values for this substance which varied within 2 per cent. This discrepancy is due to the fact that the substance does not burn completely and always leaves varying amounts of unburned carbon.

${ }_{62}\left(\mathrm{C}_{6} \mathrm{H}_{5}\right)_{2}: \mathrm{C}: \stackrel{\mathrm{H}}{\mathrm{C}}-\mathrm{C}-\mathrm{C}_{6} \mathrm{H}_{5}$.

26. QUINONES

$Q=26.05 \times N+33.1-3.5 a-6.5 b$

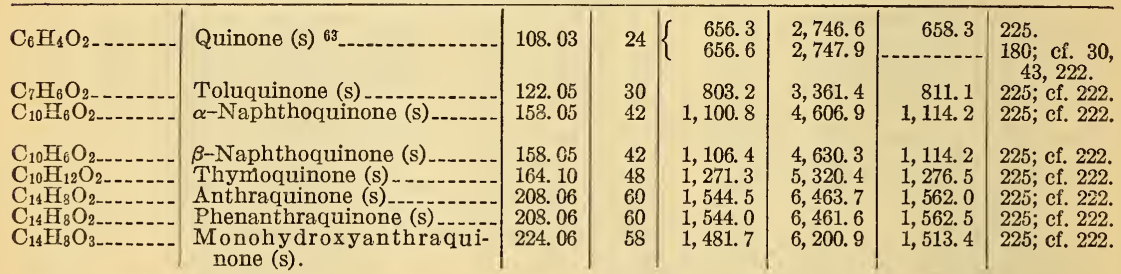

63 The heat of combustion of quinhydrone is given by (180), Z. Phys. Chem., 117, p. 57, 1925, as 1,334.5 kg-cal 15 per molo. 


\section{TABLES OF DATA-Continued}

\section{CHO COMPOUNDS-Continued}

26. QUINONES-Continued

$Q=26.05 \times N+33.1-3.5 a-6.5 b$

\begin{tabular}{|c|c|c|c|c|c|c|c|}
\hline Formula & Name & $\begin{array}{c}\text { Molec- } \\
\text { ular } \\
\text { weight }\end{array}$ & $\begin{array}{l}\text { Num- } \\
\text { ber } \\
\text { of } \\
\text { elec- } \\
\text { trons } \\
(N)\end{array}$ & $\begin{array}{l}\text { Kg-cal } 15 \\
\text { (experi- } \\
\text { mental) }\end{array}$ & $\begin{array}{l}\text { Kilo- } \\
\text { joules } \\
\text { (K.J.) }\end{array}$ & $\begin{array}{l}\mathrm{Kg} \text {-cal } \\
\text { (calcu- } \\
\text { lated to } \\
\text { the } \\
\text { liquid } \\
\text { state) }\end{array}$ & Literature \\
\hline $\mathrm{C}_{14} \mathrm{H}_{8} \mathrm{O}_{4}$ & 1,2-Dihydroxyanthraqui- & 240.06 & 56 & $1,448.9$ & $6,063.6$ & $1,464.8$ & 225 ; cf. 222. \\
\hline $\mathrm{C}_{14} \mathrm{Fi}_{8} \mathrm{O}_{5}$ & 1,2,4-Trihydroxyanthraqui- & 256.06 & $5 \frac{4}{4}$ & $1,402.1$ & $5,867.8$ & $1,416.2$ & 225 ; cf. 222. \\
\hline $\mathrm{C}_{14} \mathrm{H}_{8} \mathrm{O}_{8--}$ & $\begin{array}{l}\text { 1, 2, 3, 5, 6, 7-Hexahy droxy- } \\
\text { anthraquinone (s) (Rufi- }\end{array}$ & 304.06 & 48 & $1,249.1$ & $5,227.5$ & $1,268.4$ & 225 ; cf. 222. \\
\hline $\mathrm{C}_{18} \mathrm{H}_{16} \mathrm{O}_{2--}$ & $\begin{array}{l}\text { Retenequinone (s) (Alethyl- } \\
\text { isopropylphenanthraqui- } \\
\text { none). }\end{array}$ & 264.13 & 84 & $2,152.4$ & $9,007.8$ & $2,187.7$ & 225 ; cf. 222 \\
\hline
\end{tabular}

\section{HYDROAROMATIC AND POLYMETHYLENE KETONES}

$Q=26.05 \times N+6.5$

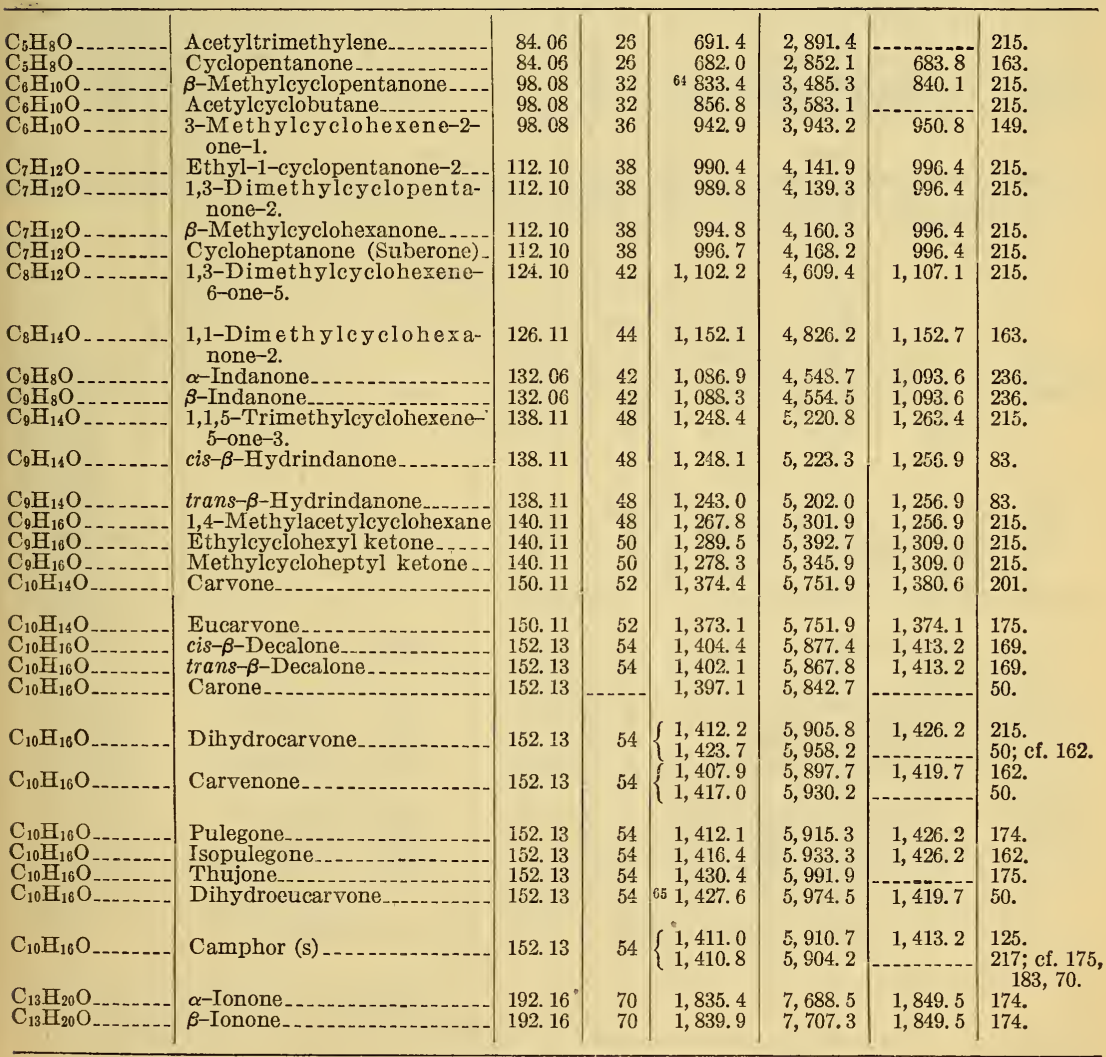

64 The correction of 1 per cent applied to (215) values is evidently too large. Roth recommends about 0.6 per cent

${ }_{66}^{6}$ The author (50) gives values about 0.6 per cent too high. 


\section{TABLES OF DATA-Continued}

\section{CHO COMPOUNDS-Continued}

28. CARBOHYDRATES (Monosaccharides)

$Q=26.05 \times N+13 q+13 \mathbf{j}+6.5 \mathrm{k}+3.5 \mathrm{I}$

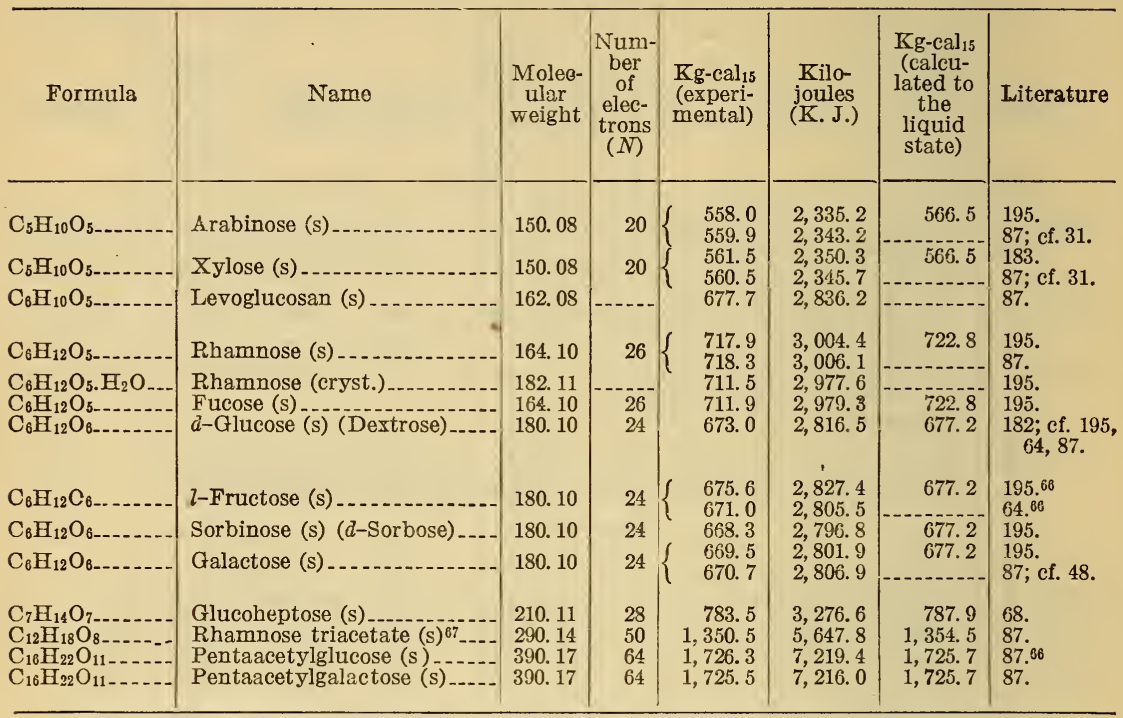

${ }^{66}$ The heat of combustion of benzoic acid obtained by these investigators is about 0.2 per cent higher than the present international value. Their results are therefore uniformly too high.

67 For the method of calculating these compounds, consult the formula for esters.

\section{DISACCHARIDES}

$Q=26.05 \times N+19.50+13 \mathrm{j}+6.5 \mathrm{k}+3.5 \mathrm{l}+13 \mathrm{q}$

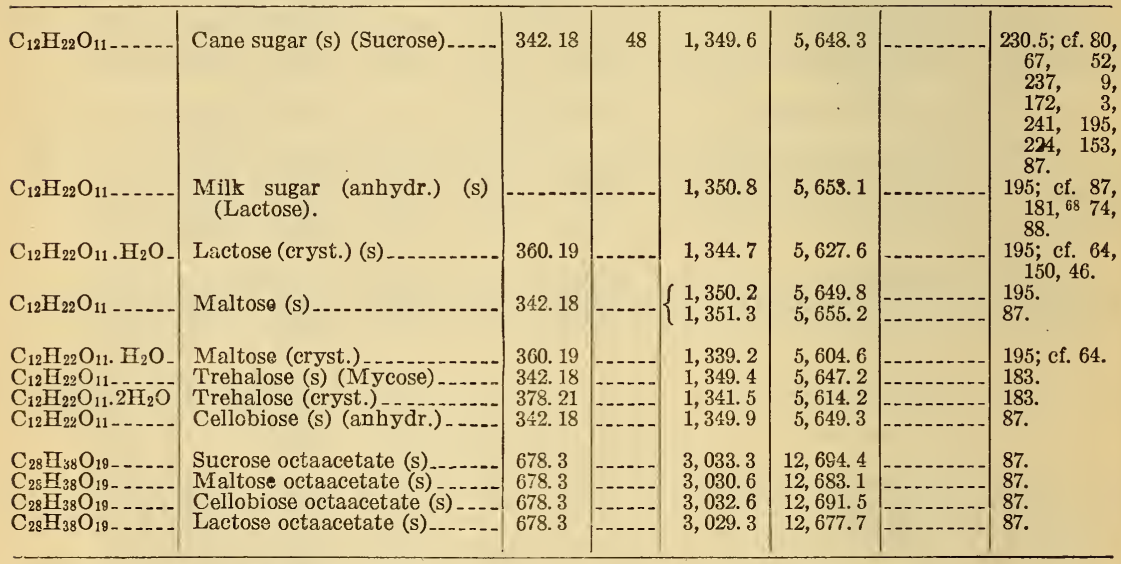

68 The author (181) used the potassium chlorate method. The value is unreliable. 


\section{TABLES OF DATA-Continued}

\section{CHO COMPOUNDS-Continued}

30. TRI, TETRA, AND POLYSACCHARIDES

\begin{tabular}{|c|c|c|c|c|c|c|c|}
\hline Formula & Name & $\begin{array}{l}\text { Molec- } \\
\text { ular } \\
\text { weight }\end{array}$ & $\begin{array}{c}\text { Num- } \\
\text { ber } \\
\text { of } \\
\text { elec- } \\
\text { trons } \\
(N)\end{array}$ & $\begin{array}{l}\mathrm{Kg} \text {-cal }{ }_{15} \\
\text { (experi- } \\
\text { mental) }\end{array}$ & $\begin{array}{c}\text { Kilo- } \\
\text { joules } \\
\text { (K. J.) }\end{array}$ & $\begin{array}{c}\mathrm{Kg}-\mathrm{cal}_{15} \\
\text { (calcu- } \\
\text { lated to } \\
\text { the } \\
\text { liquid } \\
\text { state) }\end{array}$ & Eiterature \\
\hline $\begin{array}{l}\mathrm{C}_{18} \mathrm{H}_{32} \mathrm{O}_{16} \\
\mathrm{C}_{18} \mathrm{H}_{32} \mathrm{O}_{16} 5 \mathrm{H}_{2} \mathrm{O} \\
\mathrm{C}_{18} \mathrm{H}_{32} \mathrm{O}_{16} \cdot \mathrm{H}_{2} \mathrm{O} \\
\mathrm{C}_{24} \mathrm{H}_{42} \mathrm{O}_{21}\end{array}$ & $\begin{array}{l}\text { Raffinose (s) (Melitose) } \\
\text { Raffinose (cryst.) } \\
\text { Melezitose (s) } \\
\text { Stachyose (anhydrous) (s) } \\
\text { calc. } \\
\text { Starch. } \\
\text { Starch acetate. } \\
\text { Inulin } \\
\text { Inulin acetate. } \\
\text { Dextrin } \\
\text { Glycogen } 60 \\
\text { Cellulose } \\
\text { Cellulose acetate } \\
\text { Xylan } \\
\text { Xylan acetate. }\end{array}$ & $\begin{array}{l}504.26 \\
594.34 \\
522.28 \\
664.34\end{array}$ & & $\begin{array}{r}2,025.5 \\
2,018.9 \\
2,042.0 \\
2,708.7 \\
4,178.8 \\
4,499.0 \\
4,129.9 \\
4,190.0 \\
4,522 \\
4,107.9 \\
4,185.8 \\
4,180.8 \\
4,496.0 \\
4,243.0 \\
4,548.0\end{array}$ & $\begin{array}{r}8,476.7 \\
8,449.1 \\
8,545.8 \\
11,335.9 \\
17,488.3 \\
18,828.3 \\
17,283.6 \\
17,535.2 \\
18,924.6 \\
17,191.6 \\
17,521.8 \\
17,496.6 \\
18,815.8 \\
17,756.9 \\
19,033.4\end{array}$ & (1) & $\begin{array}{l}195 ; \text { cf. } 31 . \\
195 . \\
195 . \\
87 . \\
195.69 \\
87 . \\
195 . \\
87 . \\
87 . \\
195 . \\
207 \text {; cf. } 64 \text {, } \\
75 \text {, } 133 . \\
195 ; \text { cf. } 76 . \\
87 . \\
87 . \\
87.71\end{array}$ \\
\hline
\end{tabular}

$60 \mathrm{~W} . \mathrm{K}$. Slater gives as the heat of combustion of the dry monohydrate $\mathrm{C}_{6} \mathrm{H}_{10} \mathrm{O}_{5} . \mathrm{H}_{2} \mathrm{O}, 3,836 \times 4.182$ joules per gram; Biochem. J., 18, p. 629; 1924. The authors (133), on the other hand, report the following values of the mean heats of combustion of glycogen from Mytilus and from frog muscle, per gram: Anhydrous glycogen, 4,238; hydrate 4,214; dissolved glycogen, 4,202.

70 More probable value, $4,260.0$

71 These authors also give the values for diamylose, $\alpha$-tetramylose, $\beta$-hexamylose, $\alpha$-octamylose.

31. ALIPHATIC ACIDS 72

(Saturated monobasic)

$Q=26.05 \times N$

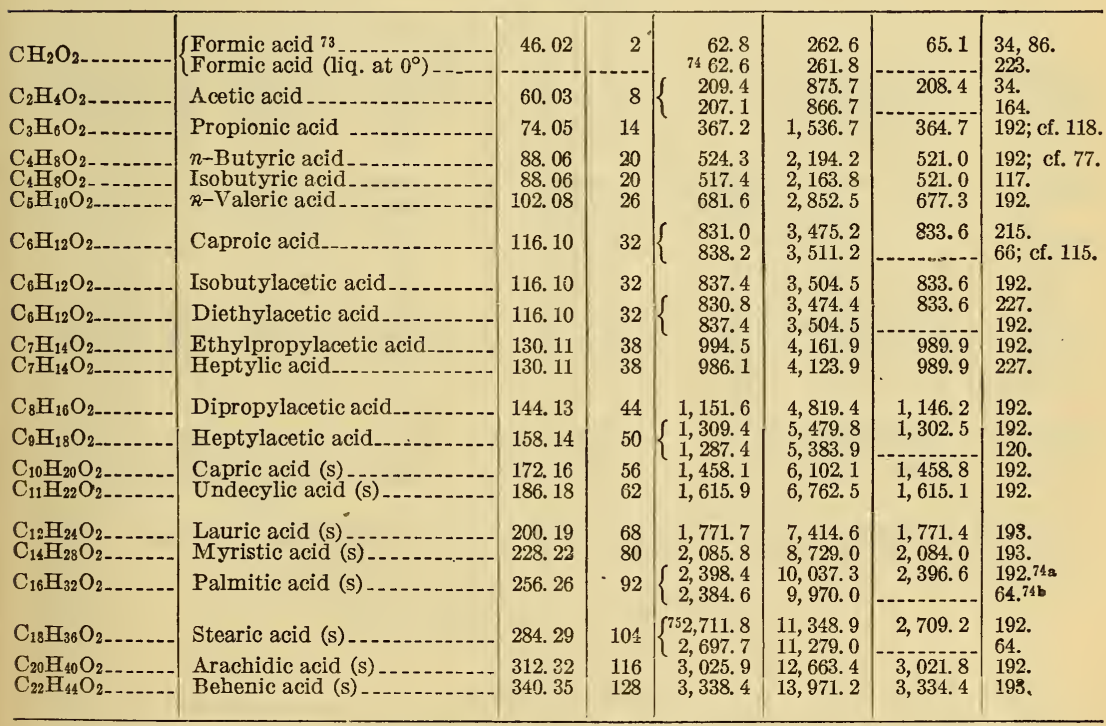

72 The values of (192) are uniformly about 0.5 to 0.7 per cent higher than the values for the same compounds recorded by modern investigators.

${ }_{73}$ The formula for formic and oxalic acids is $Q=26.05 \times N+13$.

74 This value is calculated from Thomsen's data. The figure is more or less uncertain, for the specific heat value (4.77 cal.) varies considerably with the temperature.

74a See footnote 72

74b The values of (64) are uniformly 0.2 per cent high, for the value they employed for benzoic acid was larger than the accepted one by that amount.

${ }^{73}$ See footnote 72 . 


\section{TABLES OF DATA-Continued}

\section{CHO COMPOUNDS-Continued}

32. ALIPHATIC ACIDS

(Hydroxy and Keto Ac:ds)

$Q=26.05 \times N+6.5 \mathrm{~s}+13 \mathrm{j}+6.5 \mathrm{k}+3.5 \mathrm{l}+13 \mathrm{u}+6.5 \mathrm{v}+6.5 \mathrm{w}$

\begin{tabular}{|c|c|c|c|c|c|c|c|}
\hline Formula & Name & $\begin{array}{l}\text { Molec- } \\
\text { ular } \\
\text { weight }\end{array}$ & $\begin{array}{l}\text { Num- } \\
\text { ber } \\
\text { of } \\
\text { elec- } \\
\text { trons }\end{array}$ & $\begin{array}{l}\mathrm{Kg} \text {-cal } \\
\text { (experi- } \\
\text { mental) }\end{array}$ & $\begin{array}{c}\text { Kilo- } \\
\text { joules } \\
\text { (K. J.) }\end{array}$ & $\begin{array}{c}\text { Kg-cal } 15 \\
\text { (calcu- } \\
\text { lated to } \\
\text { the } \\
\text { liquid } \\
\text { state) }\end{array}$ & Literature \\
\hline $\mathrm{C}_{2} \mathrm{H}_{2} \mathrm{O}_{3} \cdot \mathrm{H}_{2} \mathrm{C}$ & Dihydroxyacetic acid (s) & 92.03 & 4 & ${ }^{76} 125.5$ & 524.8 & 130.2 & 35. \\
\hline $\mathrm{C}_{2} \mathrm{H}$ & Glycollic acid (s) & 76.03 & 6 & 166.6 & 697.2 & 169.3 & \\
\hline & Pyroracemic acid....- & 88.03 & 10 & 279.1 & 1,1 & 280.5 & 49. \\
\hline $\mathrm{C}_{3}$ & Lactic acid (s) & 90.05 & 12 & 77326.0 & $1,363.0$ & 325.6 & $\begin{array}{l}\text { 64; cf. 127, } \\
135,75 \text {, }\end{array}$ \\
\hline $\mathrm{C}_{4} \mathrm{H}_{8} \mathrm{O}_{3}$ & Hydroxyisobutyric acid (s) & 104.06 & 18 & 471.8 & $1,973.1$ & 481.9 & $\begin{array}{l}134.10 \\
134 ; \text { cf. } 127 .\end{array}$ \\
\hline $\mathrm{C}_{4} \mathrm{H}_{8} \mathrm{O}_{3}$ & $\begin{array}{l}\beta \text {-Hydroxybutyric acid (s) } \\
\text { (Racemic). }\end{array}$ & 104.06 & 18 & 487.9 & $2,039.9$ & 475.4 & 64. \\
\hline $\mathrm{C}_{5} \mathrm{H}_{4} \mathrm{C}$ & Pyromucic acid (s) & 112.03 & & 489.7 & & & 182 ; cf. 44. \\
\hline & Levulinic acid (s) ( $\beta$-Aceto- & 116. 06 & 22 & 576.8 & $2,412.2$ & 579.6 & 18. \\
\hline $\mathrm{C}_{22} \mathrm{H}_{44} \mathrm{O}_{4}$ & Dihydroxybehenic acid (s) -. & 372.35 & 124 & $3,235.7$ & $13,541.4$ & $3,243.2$ & 193. \\
\hline
\end{tabular}

${ }_{76}$ See method of calculation for formic and oxalic acids, footnote 73 .

77 This value was obtained by burning a concentrated solution of lactic acid and analyzing the same for carbon and hydrogen.

78 The value was obtained by burning the ethyl and methyl esters of lactic acid.

53. ALIPHATIC ACIDS (UNSATURATED)

$Q=26.05 \times N+13$

\begin{tabular}{|c|c|c|c|c|c|c|c|}
\hline $\mathrm{C}_{3} \mathrm{H}_{4} \mathrm{O}_{2}--$ & Acrylic acid. & 73.02 & 12 & 79327.5 & $1,369.6$ & 325.6 & 140 ; cf. 155 . \\
\hline $\mathrm{C}_{4} \mathrm{H}_{6} \mathrm{O}_{2}$ & Crotonic acid (s) & 86.05 & 18 & 477.7 & $1,999.2$ & 481.9 & 183. \\
\hline $\mathrm{C}_{5} \mathrm{H}_{8} \mathrm{O}_{2-}$ & Tiglic acid (s) & 100.06 & 24 & 626.4 & $2,621.5$ & 631.7 & 183. \\
\hline $\mathrm{C}_{5} \mathrm{H}_{8} \mathrm{O}_{2-}$ & Angelic acid (s) & 100.06 & 24 & 80634.8 & $2,656.6$ & 638.2 & 183. \\
\hline $\mathrm{C}_{5} \mathrm{H}_{8} \mathrm{O}$ & $\alpha-\beta$-Pentenoic acid (s) & 100.06 & 24 & 623.7 & $2,610.2$ & 638.2 & 166. \\
\hline $\mathrm{C}_{5} \mathrm{H}_{8} \mathrm{O}_{2}$ & $\beta-\gamma$-Pentenoic acid (s) & 100.06 & 24 & 632.2 & $2,645.8$ & 638.2 & 166. \\
\hline $\mathrm{C}_{5} \mathrm{H}_{8} \mathrm{O}_{2}$ & Allylacetic acid & 100.06 & 24 & 641.6 & $2,685.1$ & 638.2 & 167. \\
\hline $\mathrm{C}_{6} \mathrm{H}_{8} \mathrm{O}$ & Sorbic acid (s) & 112. 05 & 28 & 743.0 & $3,109.5$ & 755.4 & 183. \\
\hline $\mathrm{C}_{6} \mathrm{H}_{10} \mathrm{O}_{2}$ & Hydrosorbic acic & 114.08 & 30 & 795.4 & $3,331.9$ & 794.5 & 66. \\
\hline $\mathrm{C}_{10} \mathrm{H}_{16} \mathrm{O}_{2}$ & Geranic acid.... & 168.13 & 52 & $1,379.0$ & $5,776.7$ & $1,380.6$ & 171. \\
\hline $\mathrm{C}_{11} \mathrm{H}_{2}$ & Undecylenic acid (s) & 184.16 & 60 & $811,579.7$ & $6,611.0$ & $1,576.0$ & 183. \\
\hline $\mathrm{C}_{18} \mathrm{H}_{34} \mathrm{O}$ & Oleic acid & 282.27 & 102 & $\begin{array}{l}2,657.0 \\
2,681.8\end{array}$ & $\begin{array}{l}11,108.9 \\
11,223.3\end{array}$ & $\begin{array}{r}2,663.6 \\
-\end{array}$ & $\begin{array}{l}64 . \\
183 .\end{array}$ \\
\hline $\mathrm{C}_{18} \mathrm{H}_{34} \mathrm{O}_{2 \ldots} \ldots$ & Elaidic acid (s) & 282.27 & 102 & $2,664.2$ & $11,149.7$ & $2,670.1$ & 183. \\
\hline $\mathrm{C}_{22} \mathrm{H}_{42} \mathrm{O}_{2} \ldots$ & Brassidic acid (s) & 338.34 & 126 & $3,290.1$ & $13,769.1$ & $3,288.8$ & 193. \\
\hline $\mathrm{C}_{22} \mathrm{H}_{42} \mathrm{O}_{2} \ldots$ & Erucic acid (s) & 338.34 & 126 & $3,296.7$ & $13,796.7$ & $3,295.3$ & 193. \\
\hline
\end{tabular}

79 The above is the mean of two determinations which do not agree better than 0.4 per cent.

80 For method of calculating cis-trans isomers, consult formula for maleic acid.

81 Mean value.

34. ALIPHATIC ACIDS (MONOBASIC)

(Acetylene type)

$Q=26.05 \times N+33.1 \mathrm{~h}$

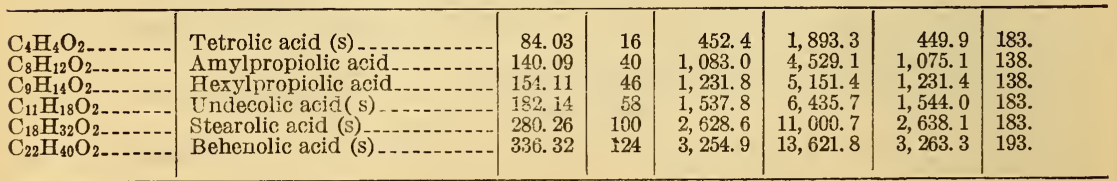




\section{TABLES OF DATA-Continued}

2. CHO COMPOUNDS-Continued

35. ALIPHATIC ACIDS (POLYBASIC SATURATED)

\begin{tabular}{|c|c|c|c|c|c|c|c|}
\hline \multicolumn{8}{|c|}{$Q=26.05 \times N$} \\
\hline Formula & Name & $\begin{array}{l}\text { Molec- } \\
\text { ular } \\
\text { weight }\end{array}$ & $\begin{array}{l}\text { Num- } \\
\text { ber } \\
\text { of } \\
\text { elec- } \\
\text { trons } \\
(N)\end{array}$ & $\begin{array}{l}\text { Kg-cal } 15 \\
\text { (experi- } \\
\text { mental) }\end{array}$ & $\begin{array}{l}\text { Kilo- } \\
\text { joules } \\
\text { (K. J.) }\end{array}$ & $\begin{array}{c}\mathrm{Kg}-\mathrm{cal}_{15} \\
\text { (calcu- } \\
\text { lated to } \\
\text { the } \\
\text { liquid } \\
\text { state) }\end{array}$ & Literature \\
\hline $\mathrm{C}_{2} \mathrm{H}_{2} \mathrm{O}_{4-}$ & Oralic acid (s) & 90.02 & 2 & $\begin{array}{l}60.2 \\
60.1\end{array}$ & $\begin{array}{l}251.9 \\
251.5\end{array}$ & ${ }^{82} 65.1$ & 190. \\
\hline $\mathrm{C}_{3} \mathrm{H}_{4} \mathrm{O}$ & Malonic acid (s) & 104.03 & 8 & $\left\{\begin{array}{l}200.1 \\
206.5\end{array}\right.$ & $\begin{array}{l}8,671.3 \\
8,642.0\end{array}$ & 208.4 & $\begin{array}{l}235 ; \text {; ct. } 86 ; \\
235 ; \text { cf. } 127, \\
121,123\end{array}$ \\
\hline $\mathrm{C}_{4} \mathrm{H}_{6} \mathrm{O}_{4-}$ & Succinic acid (s) & 118.05 & 14 & $\left\{\begin{array}{l}356.6 \\
357.1\end{array}\right.$ & $\begin{array}{l}1,492.4 \\
1,494.5\end{array}$ & 354.7 & 190. \\
\hline $\mathrm{C}_{4} \mathrm{H}_{6} \mathrm{O}_{4}$ & Methylmalonic acid (s) & 118.05 & 14 & $\left\{\begin{array}{l}362.2 \\
365.0\end{array}\right.$ & $\begin{array}{l}1,515.8 \\
1,527.5\end{array}$ & 364.7 & $\begin{array}{l}192 . \\
190 .\end{array}$ \\
\hline $\mathrm{C}_{5} \mathrm{H}_{8} \mathrm{O}_{4-}$ & Glutaric acid (s) & 132.06 & 20 & $\begin{array}{l}514.7 \\
514.9\end{array}$ & $\begin{array}{l}2,154.0 \\
2,154.9\end{array}$ & 521.0 & $\begin{array}{l}186 \text {. } \\
235 \text {; ff } 129\end{array}$ \\
\hline $\mathrm{C}_{5} \mathrm{H}_{8} \mathrm{O}_{4}$ & Methylsuccinic acid (s) & 132.06 & 20 & $\begin{array}{l}514.9 \\
515.7\end{array}$ & $\begin{array}{l}2,154.9 \\
2,158.2\end{array}$ & 521.0 & $234 ;$ cf. 127. \\
\hline $\mathrm{C}_{5} \mathrm{H}_{8} \mathrm{O}_{4}$ & Ethylmalor & 132.06 & 20 & 517.7 & 2, & 521.0 & 19 \\
\hline $\begin{array}{l}\mathrm{C}_{5} \mathrm{H}_{8} \mathrm{C} \\
\mathrm{C}_{6} \mathrm{H}_{8} \mathrm{C}\end{array}$ & $\begin{array}{l}\text { Dimethylmalonic acid (s) } \\
\text { Tricarballylic acid (s) }\end{array}$ & $\begin{array}{l}132.06 \\
170.06\end{array}$ & $\begin{array}{l}20 \\
20\end{array}$ & $\begin{array}{l}515.1 \\
516.0\end{array}$ & $\begin{array}{l}2,155.7 \\
2,159.5\end{array}$ & $\begin{array}{l}521.0 \\
521.0\end{array}$ & $\begin{array}{l}190 . \\
127\end{array}$ \\
\hline $\mathrm{C}_{6} \mathrm{H}_{10} \mathrm{O}_{4-}$ & Adipic acid (s) & 146.08 & 26 & $\begin{array}{l}668.6 \\
669.0\end{array}$ & $\begin{array}{l}2,798.1 \\
2,799.8\end{array}$ & 677.3 & $\begin{array}{l}190 . \\
235 .\end{array}$ \\
\hline $\mathrm{C}_{6} \mathrm{H}_{10} \mathrm{O}_{4}$ & $\alpha$-Methylglutaric acid (s) & 146.08 & 26 & 670.6 & $2,806.5$ & 677. 3 & 190. \\
\hline $\mathrm{C}_{6} \mathrm{H}_{10} \mathrm{O}_{4}$ & Ethylsuccinic acid (s) & 146.08 & 26 & & & 677.3 & 190. \\
\hline $\mathrm{C}_{6} \mathrm{H}_{10} \mathrm{O}_{4}$ & Sym. Dimethylsuccinic acid & 146.08 & 26 & 670.6 & $2,806.5$ & 677.3 & 190. \\
\hline $\mathrm{C}_{6} \mathrm{H}_{10} \mathrm{O}$ & $\begin{array}{l}\text { Sym. Dimethylsuccinic acid } \\
\text { (s) (anti-acid). }\end{array}$ & 146.08 & 26 & 674.2 & $2,821.5$ & & 183. \\
\hline $\mathrm{C}_{6} \mathrm{I}$ & $\begin{array}{l}\text { Sym. Dimethylsuccinic acid } \\
\left.\text { (s) (M. P. } 128^{\circ}\right)(\text { Racemic) }\end{array}$ & 146.08 & 26 & 671.5 & $2,810.2$ & 677.3 & 234. \\
\hline $\mathrm{C}_{6} \mathrm{H}_{10} \mathrm{O}_{4}$ & $\begin{array}{l}\text { Sym. Dimethylsuccinic acid } \\
\text { (s) (M. P. 208 }(\text { anti). }\end{array}$ & 146. 08 & 26 & 673.0 & $2,816.5$ & 677.3 & 234. \\
\hline $\mathrm{C}_{6} \mathrm{H}_{10} \mathrm{O}_{4-}$ & $\begin{array}{l}\text { Unsym. Dimethylsuccinic } \\
\text { acid (s). }\end{array}$ & 146.08 & 26 & $\begin{array}{l}671.4 \\
670.6\end{array}$ & $\begin{array}{l}2,809.8 \\
2,806.5\end{array}$ & 677.3 & $\begin{array}{l}190 . \\
234 .\end{array}$ \\
\hline $\mathrm{C}_{6} \mathrm{H}_{10} \mathrm{O}_{4}$ & Methylethylmalonic acid (s) & 146.08 & 26 & $\begin{array}{r}671.9 \\
82 \\
675.6\end{array}$ & & 677.3 & $\begin{array}{l}190 . \\
192 .\end{array}$ \\
\hline $\begin{array}{l}\mathrm{C}_{6} \mathrm{H}_{10} \mathrm{O} \\
\mathrm{C}_{6} \mathrm{H}_{10} \mathrm{O}\end{array}$ & $\begin{array}{l}\text { Propylmalonic acid (s) } \\
\text { Isopropylmalonic acid (s) }----\end{array}$ & $\begin{array}{l}146.08 \\
146.08\end{array}$ & $\begin{array}{l}26 \\
26\end{array}$ & $\begin{array}{l}83676.1 \\
83676.1\end{array}$ & $\begin{array}{l}2,829.5 \\
2,829.5\end{array}$ & $\begin{array}{l}677.3 \\
677.3\end{array}$ & 192. \\
\hline $\mathrm{C}_{7} \mathrm{~F}$ & $\begin{array}{l}\text { Pimelic acid (s) (Isopropyl- } \\
\text { succinic acid). }\end{array}$ & 160.04 & 32 & $\begin{array}{l}822.3 \\
827.5 \\
827.7\end{array}$ & $\begin{array}{l}3,441.3 \\
3,463.0 \\
3,463.9\end{array}$ & $\begin{array}{c}833.6 \\
-\cdots---\end{array}$ & $\begin{array}{l}190 . \\
187 . \\
235 .\end{array}$ \\
\hline $\mathrm{C}_{7} \mathrm{H}_{12} \mathrm{O}$ & Diethylmalonic acid (s) & 160.10 & 32 & 83832.6 & $3,484.4$ & 833.6 & 192. \\
\hline & Trimethylsuccinic acid (s) -- & 160.10 & 32 & $\begin{array}{l}829.9 \\
985.2\end{array}$ & $\begin{array}{l}3,473.1 \\
4,123.0\end{array}$ & $\begin{array}{l}833.6 \\
989.9\end{array}$ & $\begin{array}{l}234 . \\
190 .\end{array}$ \\
\hline $\mathrm{C}_{8} \mathrm{H}_{14} \mathrm{O}_{4}$ & Suberic acid (s) & 174.11 & 38 & $\begin{array}{l}983.3 \\
983.4\end{array}$ & $\begin{array}{l}4,115.1 \\
4,115.5\end{array}$ & 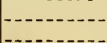 & 187. \\
\hline $\mathrm{C}_{8} \mathrm{H}_{14} \mathrm{O}_{4-}$ & $\begin{array}{l}\text { Dimethyladipic acid (s) } \\
(\text { sym.). }\end{array}$ & 174.11 & 38 & 986.7 & $4,129.3$ & 989.9 & 182 \\
\hline $\mathrm{C}_{8} \mathrm{H}_{14} \mathrm{O}_{4-}$ & $\begin{array}{l}\text { Diethylsuccinic acid (s) } \\
\text { (unsym.). }\end{array}$ & 174.11 & 38 & 984.6 & $4,120.6$ & 989.9 & 234. \\
\hline $\mathrm{C}_{8} \mathrm{H}_{14} \mathrm{O}_{4-}$ & $\begin{array}{l}\text { Diethylsuccinic acid } \\
(\text { sym. })\left(\mathrm{M} \text {. P. } 128^{\circ}\right) \text {. }\end{array}$ & 174.11 & 38 & 986.1 & $4,126.8$ & 989.9 & 234. \\
\hline $\mathrm{C}_{8} \mathrm{H}_{14} \mathrm{O}$ & $\begin{array}{l}\text { Diethylsuccinic acid } \\
\text { (sym.) (M. P. } 192^{\circ} \text { ). }\end{array}$ & 174.11 & 38 & 987.8 & $4,133.9$ & 989.9 & 234. \\
\hline $\begin{array}{l}\mathrm{C}_{8} \mathrm{H}_{14} \mathrm{O}_{4} \\
\mathrm{C}_{8} \mathrm{H}_{14} \mathrm{O}_{4-}\end{array}$ & $\begin{array}{l}\text { Ethylpropylmalonic acid (s) } \\
\text { Tetramethylsuccinic acid (s) }\end{array}$ & $\begin{array}{l}174.11 \\
174.11\end{array}$ & $\begin{array}{l}38 \\
38\end{array}$ & $\begin{array}{r}84988.5 \\
989.4\end{array}$ & $\begin{array}{l}4,136.9 \\
4,140.6\end{array}$ & $\begin{array}{l}989.9 \\
989.9\end{array}$ & 192. \\
\hline $\mathrm{C}_{9} \mathrm{H}_{16} \mathrm{O}_{4--}$ & Azelaic acid (s) & 188.13 & 44 & $\left\{\begin{array}{l}1,141 \cdot 0 \\
1,141 \cdot 7\end{array}\right.$ & $\begin{array}{l}4,775.1 \\
4,778.0\end{array}$ & $1,146.2$ & $\begin{array}{l}191 . \\
235 .\end{array}$ \\
\hline $\mathrm{C}_{9} \mathrm{H}_{16} \mathrm{O}_{4}--$ & Dipropylmalonic acid (s) & 188.13 & 44 & $851,145.8$ & $4,795.2$ & $1,146.2$ & 192. \\
\hline
\end{tabular}

82 The calculated heats of combustion for compounds in which 2 weakly electronegative groups, such as COOH, are linked together, is given by the expression $Q=26.05 \times N+13$. The correction factor thus denotes that the two carbon atoms share a pair of valence electrons in outer energy levels, compared to a carbon-to-carbon linkage as in ethane.

s3 The values of (192) are on the whole about 0.5 to 0.7 per cent too high.

81 See footnote 83 .

85 See footnote 83 . $3697^{\circ}-29-3$ 


\section{TABLES OF DATA-Continued}

\section{CHO COMPOUNDS-Continued}

35. ALIPHATIC ACIDS (POLYBASIC SATURATED)-Continued

$Q=26.05 \times N$

\begin{tabular}{|c|c|c|c|c|c|c|c|}
\hline Formula & Name & $\begin{array}{l}\text { Niolec- } \\
\text { ular } \\
\text { weight }\end{array}$ & $\begin{array}{c}\text { Num- } \\
\text { ber } \\
\text { of } \\
\text { elec- } \\
\text { trons } \\
(N)\end{array}$ & $\begin{array}{l}\mathrm{Kg}_{\text {-cal }} 15 \\
\text { (experi- } \\
\text { mental) }\end{array}$ & $\begin{array}{l}\text { Kilo- } \\
\text { joules } \\
\text { (K. J.) }\end{array}$ & $\begin{array}{c}\mathrm{Kg} \text {-cal } \\
\text { (calcu- } \\
\text { lated to } \\
\text { the } \\
\text { liquid } \\
\text { state) }\end{array}$ & Literature \\
\hline $\mathrm{C}_{10} \mathrm{H}_{18} \mathrm{O}_{4}$ & Sebacic acid (s) & 200.14 & 50 & $\left\{\begin{array}{l}1,296.4 \\
1,297.3\end{array}\right.$ & $\begin{array}{l}5,425.4 \\
5,429.2\end{array}$ & 1,302.5 & $\begin{array}{l}191 . \\
235 ; \text { cf. } 127 .\end{array}$ \\
\hline $\begin{array}{l}\mathrm{C}_{10} \mathrm{H}_{18} \mathrm{O}_{4} \ldots \ldots \\
\mathrm{C}_{10} \mathrm{H}_{18} \mathrm{O}_{4} \ldots \ldots \\
\mathrm{C}_{11} \mathrm{H}_{20} \mathrm{O}_{4} \ldots \ldots \\
\mathrm{C}_{11} \mathrm{H}_{20} \mathrm{O}_{4} \ldots \ldots\end{array}$ & $\begin{array}{l}\text { Heptylmalonic acid (s) } \\
\text { Triethylsuccinic acid (s) } \\
n \text {-Octylmalonic acid (s) } \\
\text { Nonanedicarboxylic acid (s) }\end{array}$ & $\begin{array}{l}200.14 \\
200.14 \\
216.16 \\
216.16\end{array}$ & $\begin{array}{l}50 \\
50 \\
56 \\
56\end{array}$ & $\begin{array}{r}851,302.4 \\
1,301.3 \\
851,458.1 \\
1,455.6\end{array}$ & $\begin{array}{l}5,450.5 \\
5,445.9 \\
6,102.1 \\
6,091.7\end{array}$ & $\begin{array}{l}1,302.5 \\
1,302.5 \\
1,458.8 \\
1,458.8\end{array}$ & $\begin{array}{l}192 . \\
234 . \\
192 . \\
235 .\end{array}$ \\
\hline $\begin{array}{l}\mathrm{C}_{12} \mathrm{H}_{22} \mathrm{O}_{4} \ldots \ldots \\
\mathrm{C}_{12} \mathrm{H}_{22} \mathrm{O}_{4} \ldots \ldots \\
\mathrm{C}_{13} \mathrm{H}_{24} \mathrm{O}_{4} \ldots \ldots\end{array}$ & $\begin{array}{l}\text { Decanedicarboxylic acid (s) -- } \\
\text { Tetraethylsuccinic acid (s) -- } \\
\text { Brassylic acid (s) (Undecane- } \\
\text { dicarboxylic acid). }\end{array}$ & $\begin{array}{l}230.18 \\
230.18 \\
244.19\end{array}$ & $\begin{array}{l}62 \\
62 \\
68\end{array}$ & $\begin{array}{l}1,610.7 \\
1,618.8 \\
1,768.6\end{array}$ & $\begin{array}{l}6,740.8 \\
6,774.6 \\
7,401.6\end{array}$ & $\begin{array}{l}1,615.1 \\
1,615.1 \\
1,771.4\end{array}$ & $\begin{array}{l}235 . \\
235 . \\
235 .\end{array}$ \\
\hline $\mathrm{C}_{18} \mathrm{H}_{36} \mathrm{O}_{4}$ & Cetylmalonic acid (s) & 328.29 & 104 & $562,707.3$ & $11,330.0$ & $2,709.2$ & 192. \\
\hline
\end{tabular}

85 See footnote 83 .

86 See footnote 83 .

36. HYDROXY POLYBASIC ACIDS (SATURATED)

$Q=26.05 \times N+6.5 \mathrm{k}+6.5 \mathrm{v}$

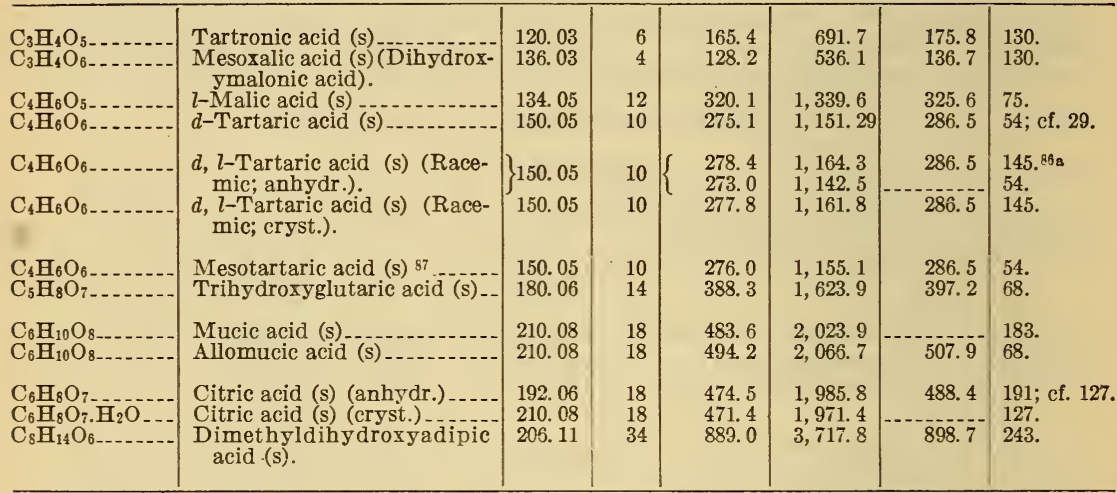

863 Little value should be attached to the work of this investigator. The results are probably not better than 1 to 2 per cent and are too high.

87 The authors (54) give also the heat of combustion of various ammonium and substituted ammonium salts of $d$-tartaric, racemic and mesotartaric acids:

\begin{tabular}{|c|c|c|c|c|c|}
\hline & $\mathrm{Kg}-\mathrm{cal}_{15}$ & K. J. & & $\mathrm{Kg}$-cal ${ }_{15}$ & K. J. \\
\hline 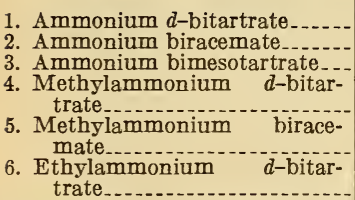 & $\begin{array}{l}341.7 \\
339.5 \\
341.2 \\
508.0 \\
506.0 \\
665.4\end{array}$ & $\begin{array}{l}1,430.0 \\
1,420.8 \\
1,427.9 \\
2,126.0 \\
2,117.6 \\
2,784.7\end{array}$ & 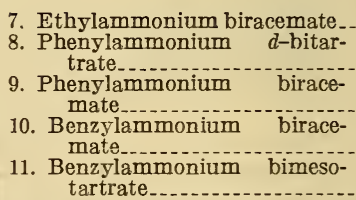 & $\begin{array}{r}663.1 \\
1,079.3 \\
1,077.3 \\
1,229.9 \\
1,231.5\end{array}$ & $\begin{array}{r}2,775.1 \\
.4,516.9 \\
4,508.5 \\
5,147.1 \\
5,153.8\end{array}$ \\
\hline
\end{tabular}




\section{TABLES OF DATA-Continued}

\section{CHO COMPOUNDS-Continued}

37. POLYBASIC ALIPHATIC ACIDS (UNSATURATED)

$Q=26.05 \times N+13 \mathrm{y}+16.5 \mathrm{x}$

\begin{tabular}{|c|c|c|c|c|c|c|c|}
\hline Formula & Name & $\begin{array}{c}\text { Molec- } \\
\text { ular } \\
\text { weight }\end{array}$ & $\begin{array}{l}\text { Num- } \\
\text { ber } \\
\text { of } \\
\text { elec- } \\
\text { trons } \\
(N)\end{array}$ & $\begin{array}{l}\mathrm{Kg} \text {-cal }{ }_{15} \\
\text { (experi- } \\
\text { mental) }\end{array}$ & $\begin{array}{c}\text { Kilo- } \\
\text { joules } \\
\text { (K. J.) }\end{array}$ & $\begin{array}{l}\mathrm{Kg}-\mathrm{cal}_{15} \\
\text { (calcu- } \\
\text { lated to } \\
\text { the } \\
\text { liquid } \\
\text { state) }\end{array}$ & Literature \\
\hline $\mathrm{C}_{4} \mathrm{H}_{4} \mathrm{O}_{4-}$ & Fumaric acid (s) 88 (trans) & 116.03 & 12 & 320.0 & $1,339.2$ & 325.6 & 190; cf. 163 , \\
\hline $\mathrm{C}_{4} \mathrm{H}_{4} \mathrm{O}_{4}$ & Maleic acid (s) (cis) & 116.03 & 12 & 326.1 & $1,364.7$ & 329.1 & 190; cf. 127, \\
\hline $\mathrm{C}_{5} \mathrm{H}_{6} \mathrm{O}_{4-}$ & $\begin{array}{l}\text { Itaconic acid (s) (Methyl- } \\
\text { enesuccinic acid). }\end{array}$ & 130.05 & 18 & $\begin{array}{l}475.6 \\
475.0\end{array}$ & $\begin{array}{l}1,990.4 \\
1,987.9\end{array}$ & 481.9 & $\begin{array}{l}183 . \\
151 ; \text { cf. } 127 .\end{array}$ \\
\hline $\mathrm{C}_{5} \mathrm{H}_{6} \mathrm{O}_{4-}$ & Citraconic acid (s) (methyl- & 130.05 & 18 & $\begin{array}{l}479.4 \\
478.8\end{array}$ & $2,006.2$ & 485.4 & 183. \\
\hline $\mathrm{C}_{5} \mathrm{H}_{6} \mathrm{O}_{4-}$ & Mesaconic acid (s) (Methyl- & 130.05 & 18 & $\begin{array}{l}478.8 \\
476.9 \\
475.9\end{array}$ & $\begin{array}{l}2,003.8 \\
1,995.8\end{array}$ & 481.9 & $\begin{array}{l}151 ; \text { ct. } 127 . \\
183 \text {. }\end{array}$ \\
\hline $\mathrm{C}_{6} \mathrm{H}_{6} \mathrm{O}_{6--}$ & Aconitic acid (s) & 174.05 & 18 & 475.1 & $1,988.3$ & 481.9 & 183 ; cf. 127 . \\
\hline $\begin{array}{l}\mathrm{C}_{6} \mathrm{H}_{8} \mathrm{O}_{4} \\
\mathrm{C}_{6} \mathrm{H}_{8} \mathrm{O}_{4} \ldots \\
\mathrm{C}_{6} \mathrm{H}_{8} \mathrm{O}_{4} \\
\mathrm{C}_{7} \mathrm{H}_{10} \mathrm{O}_{4}\end{array}$ & 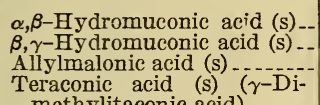 & $\begin{array}{l}\text { 144. } 06 \\
144.06 \\
144.06 \\
158.08\end{array}$ & $\begin{array}{l}24 \\
24 \\
24 \\
30\end{array}$ & $\begin{array}{r}628.8 \\
629.1 \\
\text { s9 } 637.8 \\
796.1\end{array}$ & $\begin{array}{l}2,631.5 \\
2,632.8 \\
2,669.2 \\
3,331.7\end{array}$ & $\begin{array}{l}638.2 \\
638.2 \\
638.2 \\
794.5\end{array}$ & $\begin{array}{l}183 . \\
183 . \\
192 . \\
145 .\end{array}$ \\
\hline $\mathrm{C}_{4} \mathrm{H}_{2} \mathrm{O}_{4}$ & $\begin{array}{l}\text { methylitaconic acid). } \\
\text { Acetylenedicarboxylic acid } \\
\text { (s). }\end{array}$ & 114. 02 & 10 & 305.9 & $1,280.2$ & & 183. \\
\hline
\end{tabular}

${ }^{88}$ A collection of the heats of combustion of some stereoisomeric acids is given by Liebermann, Ber., 25 , p. $90 ; 1892$.

81 The values of (192) are from 0.5 to 0.7 per cent too high.

\section{AROMATIC ACIDS}

$Q=26.05 \times N-3.5 \mathrm{a}-6.5 \mathrm{~b}$

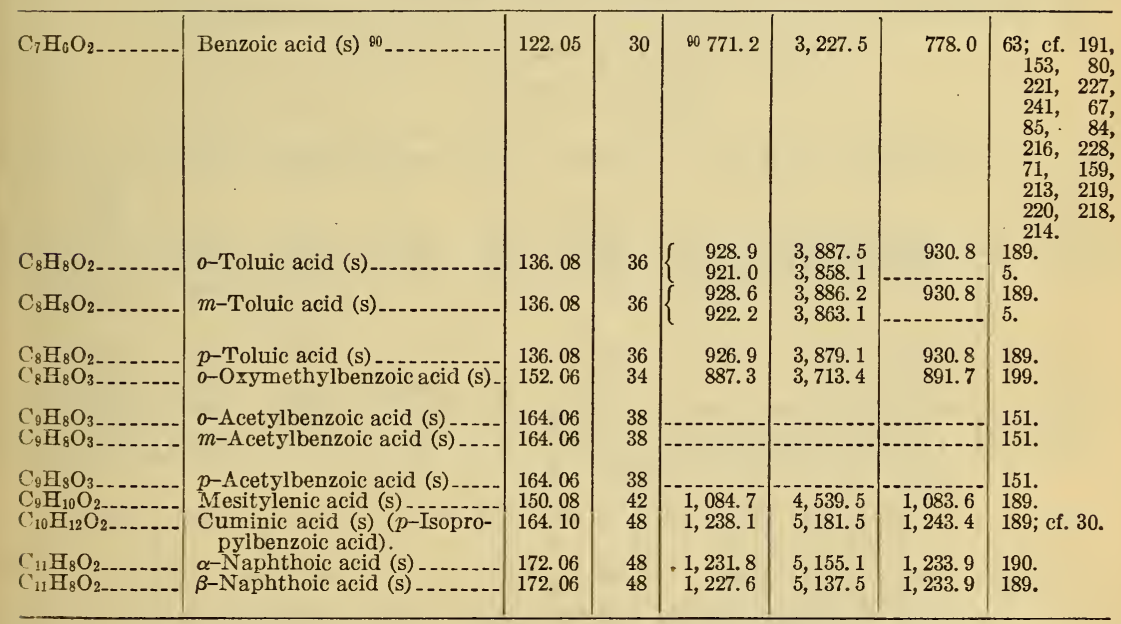

90 The above value is the one accepted at the third conference of the International Union of Pure and A pplied Chemistry, held at Lyons, 1923 . It is the value found by Dickinson, Bull. Bur. Stds., 11, p. 189; 1915. See also discussion of Verkade in Chem. Weekblad, 19, p, 389; 1922. 


\section{TABLES OF DATA-Continued}

\section{CHO COMPOUNDS-Continued}

\section{HYDROXY AROMATIC ACIDS}

$Q=26.05 \times N+3.5 m-3.5 a-6.5 b$

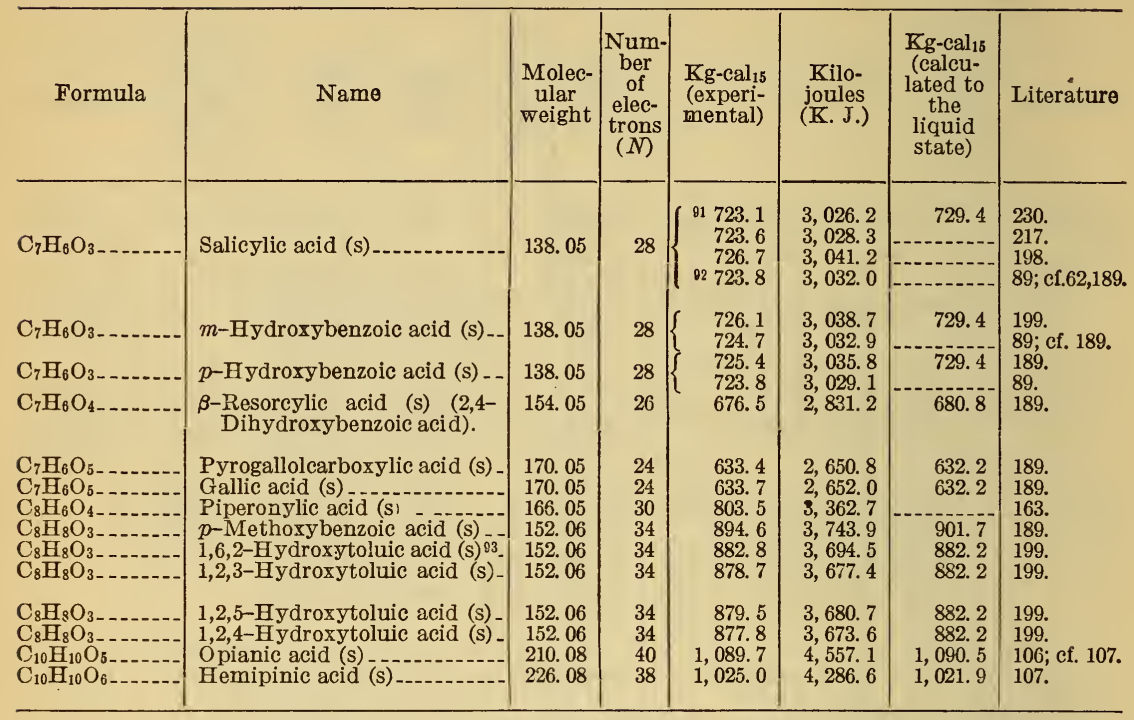

21 As a result of a very exhaustive and painstaking investigation, these authors recommend the use of salicylic acid as a secondary thermochemical standard. Compare Verkade and Coops, Bull. soc. chim., 37 , p. 1536, 1925; Verkade and Coops, J. Chem. Soc., p. 1437, 1926; and particularly Cohen, Verkade, Miyaki, Coops and van der Hoeve, Verslag Akad. Wetenschappen Amsterdam, 35, p. 48; 1926.

${ }_{22}$ The values of these authors have been corrected to the $15^{\circ}$ calorie.

${ }_{03}$ The numbers denote the positions of carboxyl, hydroxyl and methyl groups, respectively.

40. PHENYLATED ALIPHATIC ACIDS

$Q=26.05 \times N-3.5 \mathrm{a}-6.5 \mathrm{~b}$

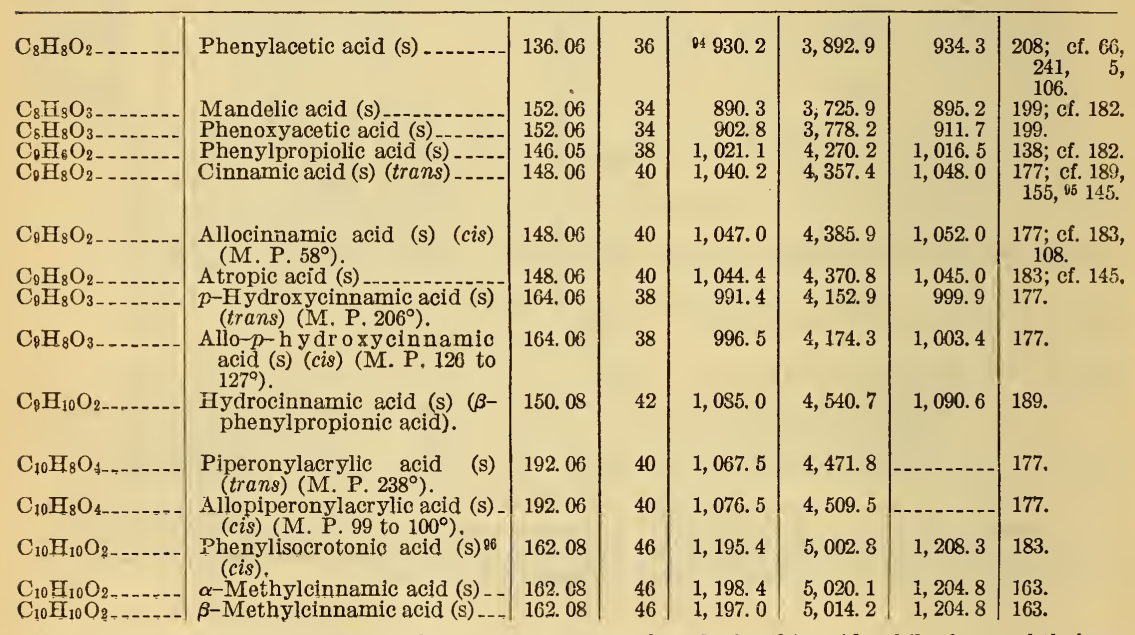

${ }^{01}$ It is most peculiar that these authors obtained $1,042.8 \mathrm{~kg}$-cal 15 for this acid while for naphthalene, which was used in standardizing the bomb, their value is 0.9 per cent higher than the accepted value.

${ }_{25}$ One of these authors showed later (Ber., 35, p. 2908, 1902), that cinnamic acid upon illumination goes over to a $\alpha$-truxillia acid. However, upon combustion there was no evidence of any energy difierence. See also Ber., 28, p. 1443; 1895; 46, p. 267; 1913.

66 $\mathrm{C}_{6} \mathrm{H}_{5} . \mathrm{CH}: \mathrm{CH}, \mathrm{CH}_{2} \mathrm{COOH}$. 


\section{TABLES OF DATA-Continued}

\section{CHO COMPOUNDS-Continued}

40. PHENYLATED ALIPHATIC ACIDS-Continued

$Q=26.05 \times N-3.5 \mathrm{a}-6.5 \mathrm{~b}$

\begin{tabular}{|c|c|c|c|c|c|c|c|}
\hline Formula & Name & $\begin{array}{c}\text { Molec- } \\
\text { ular } \\
\text { weight }\end{array}$ & $\begin{array}{c}\text { Num- } \\
\text { ber } \\
\text { of } \\
\text { elec- } \\
\text { trons } \\
(N)\end{array}$ & $\begin{array}{l}\mathrm{Kg} \text {-cal } 15 \\
\text { (experi- } \\
\text { mental) }\end{array}$ & $\begin{array}{l}\text { Kilo- } \\
\text { joules } \\
(\mathrm{K} . \mathrm{J} .)\end{array}$ & $\begin{array}{c}\mathrm{Kg} \text {-cal } \\
\text { (calcu- } \\
\text { lated to } \\
\text { the } \\
\text { liquid } \\
\text { state) }\end{array}$ & Literature \\
\hline $\mathrm{C}_{10} \mathrm{H}_{10} \mathrm{O}_{3}$ & Methylcoumaric acid (s) & 170.08 & 44 & $1,161.0$ & $4,863.4$ & $1,152.5$ & 177. \\
\hline $\mathrm{C}_{10} \mathrm{H}_{10} \mathrm{O}_{3}$ & Methylcoumarinic acid (s) & 170.08 & 44 & $1,167.2$ & $4,889.4$ & $1,156.0$ & 177. \\
\hline $\mathrm{C}_{10} \mathrm{H}_{10} \mathrm{O}_{3}$ & p-Methoxycinnamic acid & 170.08 & 44 & $1,163.0$ & $4,871.8$ & $1,172.2$ & 177. \\
\hline $\mathrm{C}_{10} \mathrm{H}_{10} \mathrm{O}_{3}$ & $\begin{array}{l}\text { Allo-p-methoxycinnamic } \\
\left.\text { acid (s) (cis) (M. P. } 66^{\circ}\right) \text {. }\end{array}$ & 170.08 & 44 & $1,172.2$ & $4,910.4$ & $1,175.7$ & 177. \\
\hline $\mathrm{C}_{11} \mathrm{H}_{10} \mathrm{O}_{2}$ & $\begin{array}{l}\text { Cinnamylideneacetic acid } \\
(\mathrm{s})\left(\mathrm{M} . \mathrm{P} .165^{\circ}\right) .\end{array}$ & 174.08 & 50 & $991,310.6$ & $5,480.9$ & $1,322.0$ & 155. \\
\hline $\mathrm{C}_{11} \mathrm{H}_{10} \mathrm{O}_{2}$ & $\begin{array}{l}\text { Allocinnamylideneacetic acid } \\
\text { (s) }\left(\mathrm{M} . \mathrm{P} .138^{\circ}\right)\end{array}$ & 174.08 & 50 & $1,319.4$ & $5,517.7$ & $1,325.5$ & 155. \\
\hline $\mathrm{C}_{11} \mathrm{H}_{10} \mathrm{O}_{4-}$ & Acetylcoumaric acid (s) & 206.08 & 46 & $1,207.6$ & $5,058.6$ & $1,217.8$ & 177. \\
\hline $\mathrm{C}_{11} \mathrm{H}_{10} \mathrm{O}_{4}$ & $\begin{array}{l}\text { Acetylcoumarinic acid (s) } \\
\text { (cis) (M. P. } 85^{\circ} \text { ). }\end{array}$ & 206.08 & 46 & $1,211.7$ & $5,075.8$ & $1,221.3$ & 177. \\
\hline $\begin{array}{l}\mathrm{C}_{11} \mathrm{H}_{10} \mathrm{O}_{4} \\
\mathrm{C}_{11} \mathrm{H}_{12} \mathrm{O}_{3}\end{array}$ & $\begin{array}{l}\text { Phenylparaconic acid (s) } \\
\text { Ethylcoumaric acid }(\mathrm{s}) \\
\left.\quad \text { (trans) (M. P. } 133 \text { to } 134^{\circ}\right) \text {. }\end{array}$ & $\begin{array}{l}206.08 \\
192.10\end{array}$ & $\begin{array}{l}46 \\
50\end{array}$ & $\begin{array}{l}1,195.5 \\
1,316.5\end{array}$ & $\begin{array}{l}5,003.2 \\
5,514.8\end{array}$ & $\begin{array}{l}1,204.8 \\
1,328.8\end{array}$ & $\begin{array}{l}183 . \\
177 .\end{array}$ \\
\hline $\mathrm{C}_{11} \mathrm{H}_{12} \mathrm{O}_{3}$ & Ethylcoumarinic acid (s) (cis) & 192.10 & 50 & $1,323.0$ & $5,542.0^{\circ}$ & $1,332.0$ & 177. \\
\hline $\mathrm{C}_{12} \mathrm{H}_{14} \mathrm{O}_{3}$ & $\begin{array}{l}\text { Propylcoumaric acid }(\mathrm{s}) \\
\left.\text { (trans) (M. P. } 105 \text { to } 106^{\circ}\right) \text {. }\end{array}$ & 206. 11 & 56 & $1,470.4$ & $6,159.5$ & $1,484.8$ & 177. \\
\hline $\mathrm{C}_{12} \mathrm{H}_{14} \mathrm{O}_{3}$ & Propylcoumarinic acid (s) & 206.11 & 56 & $1,476.3$ & $6,184.2$ & $1,488.3$ & 177. \\
\hline $\begin{array}{l}\mathrm{C}_{12} \mathrm{H}_{12} \mathrm{O}_{3} \\
\mathrm{C}_{12} \mathrm{H}_{12} \mathrm{O}_{3}\end{array}$ & $\begin{array}{c}\beta \text {-Benzallevulinic acid (s) } \\
\delta \text {-Benzallevulinic acid (s) }\end{array}$ & $\begin{array}{l}204.10 \\
204.10\end{array}$ & $\begin{array}{l}54 \\
54\end{array}$ & $\begin{array}{l}1,413.4 \\
1,410.1\end{array}$ & $\begin{array}{l}5,915.1 \\
5,901.3\end{array}$ & $\begin{array}{l}1,419.7 \\
1,419.7\end{array}$ & $\begin{array}{l}182 . \\
182 .\end{array}$ \\
\hline $\mathrm{C}_{13} \mathrm{H}_{16} \mathrm{O}_{3}$ & n-Butylcoumaric acid (s) & 220.13 & 62 & $1,630.6$ & $6,830.6$ & $1,641.1$ & 177. \\
\hline $\mathrm{C}_{13} \mathrm{H}_{16} \mathrm{O}_{3}$ & $\begin{array}{l}n \text {-Butylcoumarinic acid (s) } \\
\text { (cis) (M. P. } 53 \text { to } 54^{\circ} \text { ). }\end{array}$ & 220.13 & 62 & $1,637.0$ & $6,857.4$ & $1,644.6$ & 177. \\
\hline $\mathrm{C}_{14} \mathrm{H}_{18} \mathrm{O}_{3}$ & $\begin{array}{l}\text { Isoamylcoumaric acid }(\mathrm{s}) \\
\left.\text { (trans.) (M. P. } 79 \text { to } 79.5^{\circ}\right)\end{array}$ & 234.14 & 68 & $1,789.7$ & $7,497.1$ & $1,797.4$ & 177. \\
\hline $\mathrm{C}_{14} \mathrm{H}_{18} \mathrm{O}_{3}$ & $\begin{array}{l}\text { Isoamylcoumarinic acid (s) } \\
\text { (cis) }\left(\mathrm{M}, \mathrm{P}, 80 \text { to } 80.5^{\circ}\right)\end{array}$ & 234.14 & 68 & $1,791.3$ & $7,503.8$ & $1,800.9$ & 177. \\
\hline $\mathrm{C}_{14} \mathrm{H}_{12} \mathrm{O}_{2}$ & Diphenylacetic acid (s) & 212.10 & 64 & $1,651.5$ & $6,911.5$ & 1,654. 2 & 182 ; cf. 227. \\
\hline $\begin{array}{l}\mathrm{C}_{14} \mathrm{H}_{12} \mathrm{O}_{3-} \\
\mathrm{C}_{16} \mathrm{H}_{16} \mathrm{O}_{2-} \\
\mathrm{C}_{17} \mathrm{H}_{16} \mathrm{O}_{3}\end{array}$ & $\begin{array}{l}\text { Benzilic acid (s) } \\
\text { Dibenzylacetic acid (s) } \\
\beta \text {-Tolylmethoxycinnamic }\end{array}$ & $\begin{array}{l}228.10 \\
240.13 \\
268.13\end{array}$ & $\begin{array}{l}62 \\
76 \\
78\end{array}$ & $\begin{array}{l}1,618.2 \\
1,954.3 \\
2,035.2\end{array}$ & $\begin{array}{l}6,772.2 \\
8,178.8 \\
8,525.5\end{array}$ & $\begin{array}{l}1,612.1 \\
1,972.8 \\
2,047.9\end{array}$ & $\begin{array}{l}182 . \\
227 . \\
151 .\end{array}$ \\
\hline $\mathrm{C}_{17} \mathrm{H}_{18} \mathrm{O}_{3}$ & $\begin{array}{l}\beta \text {-Tolylmethoxycinnamic } \\
\text { acid (s) (labile). }\end{array}$ & 258.13 & 78 & $2,039.2$ & $8,542.2$ & $2,047.9$ & 151. \\
\hline
\end{tabular}

M. M. P. $170^{\circ}$ (liq. cryst.); clears at $185^{\circ}$.

${ }_{98}$ The work of these investigators is not quite reliable. Thus, the value of standard naphthalene used in the calibration of the bomb is about 0.9 per cent too high, and yet their value for cinnamic acid is within 0.1 per cent of the best value.

\section{POLYBASIC AROMATIC ACIDS}

$Q=26.05 \times N-3.5 \mathrm{a}-6.5 \mathrm{~b}$

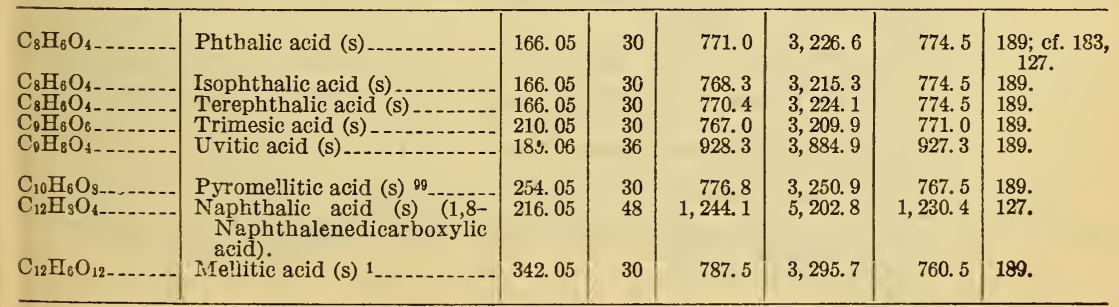

${ }^{90}$ The combustion of these acids was rather unsatisfactory. Consult original paper.

1 Ser foctnote 99 . 


\section{TABLES OF DATA-Continued}

\section{CHO COMPOUNDS-Continued}

42. PHENYLATED POLYBASIC ALIPHATIC ACIDS $Q=26.05 \times N-3.5 \mathrm{a}-6.5 \mathrm{~b}$

\begin{tabular}{|c|c|c|c|c|c|c|c|}
\hline Formula & Name & $\begin{array}{l}\text { Molec- } \\
\text { ular } \\
\text { weight }\end{array}$ & $\begin{array}{c}\text { Num- } \\
\text { ber } \\
\text { of } \\
\text { elec- } \\
\text { trons } \\
(N)\end{array}$ & $\begin{array}{l}\mathrm{Kg} \text {-cal } \\
\text { (experi- } \\
\text { mental) }\end{array}$ & $\begin{array}{l}\text { Kilo- } \\
\text { joules } \\
(\mathrm{K} . \mathrm{J} .)\end{array}$ & $\begin{array}{l}\mathrm{Kg}-\mathrm{cal}_{15} \\
\text { (calcu- } \\
\text { lated to } \\
\text { the } \\
\text { liquid } \\
\text { state) }\end{array}$ & Literature \\
\hline $\mathrm{C}_{10} \mathrm{H}_{8} \mathrm{O}_{4}$ & Benzalmalonic acid (s) & 192.06 & 40 & $1,056.0$ & $4,419.4$ & $1,048.5$ & 183. \\
\hline $\mathrm{C}_{10} \mathrm{H}_{10} \mathrm{O}_{4}$ & Benzylmalonic acid (s) & 194. 08 & 42 & $1,082.5$ & $4,530.3$ & $1,090.6$ & 192. \\
\hline $\mathrm{C}_{10} \mathrm{H}_{10} \mathrm{O}_{4}$ & Phenylsuccinic acid (s) & 194.08 & 42 & $1,082.3$ & $4,529.4$ & $1,090.6$ & 234. \\
\hline $\mathrm{C}_{12} \mathrm{H}_{10} \mathrm{O}_{4}$ & $\begin{array}{l}\text { Cinnamylidenemalonic acid } \\
\text { (s) (yellow). }\end{array}$ & 218.08 & 50 & $2 \overrightarrow{1}, 319.4$ & $5,517.7$ & $1,322.0$ & 155 . \\
\hline $\mathrm{C}_{16} \mathrm{H}_{14} \mathrm{O}_{4-}$ & $\begin{array}{l}\alpha \text {-Diphenylsuccinic acid (s) } \\
\text { (anhydr.) (easily sol. }\end{array}$ & 270.11 & 70 & $41,810.3$ & $7,576.1$ & $1,816.5$ & $182 ;$ cf. 145. \\
\hline $\mathrm{C}_{16} \mathrm{H}_{14} \mathrm{O}_{4-}$ & $\begin{array}{l}\beta \text {-Diphenylsuccinic acid (s) } \\
\text { (difficultly sol.). }\end{array}$ & 270.11 & 70 & $1,806.6$ & $7,560.3$ & $1,816.5$ & $182 ;$ cf. 145 . \\
\hline $\mathrm{C}_{15} \mathrm{H}_{16} \mathrm{O}_{4}$ & $\alpha$-Truxillic acid (s) & 296.13 & 80 & $2,083.5$ & $8,713.2$ & $2,080.0$ & 155. \\
\hline $\mathrm{C}_{24} \mathrm{H}_{20} \mathrm{O}_{8}$ & $\begin{array}{l}\text { Cinnamylidenemalonic } \\
\text { acid } 5 \text { (exposed to the ac- } \\
\text { tion of light). }\end{array}$ & 436.16 & $-\ldots$ & $2,638.6$ & $11,034.6$ & - $-\cdots-1-n$ & 155 . \\
\hline
\end{tabular}

2 See footnote 95

3 The heat of combustion of the acetone addition product of $\alpha$-diphenylsuccinic acid (easily soluble form in acetone) is given as $2,237.9 \mathrm{~kg}-\mathrm{cal}_{15}$.

4 The authors (234) report the heats of combustion of diphenylsuccinic acid (racem.) and diphenylsuccinic acid (anti) as $1,807.7 \mathrm{~kg}^{-c} \mathrm{l}_{15}$ and $1,809.0 \mathrm{~kg}^{-c_{1}} \mathrm{l}_{15}$, respectively.

${ }_{6} \mathrm{~T}$ The structure suggested for this polymer is $\mathrm{C}_{6} \mathrm{H}_{5} . \mathrm{CH} . \mathrm{CH} . \mathrm{CH}: \mathrm{C}:\left(\mathrm{CO}_{2} \mathrm{H}\right)_{2}$

\section{$\left(\mathrm{HO}_{2} \mathrm{C}\right)_{2}: \mathrm{C}: \mathrm{CH} . \stackrel{\mathrm{C}}{\mathrm{H}} \stackrel{\mathrm{I}}{\mathrm{CH}} \mathrm{H}_{6} \mathrm{H}_{5}$.}

43. HYDROAROMATIC AND POLYMETHYLENE ACIDS

$Q=26.05 \times N+13 z+13 a a$

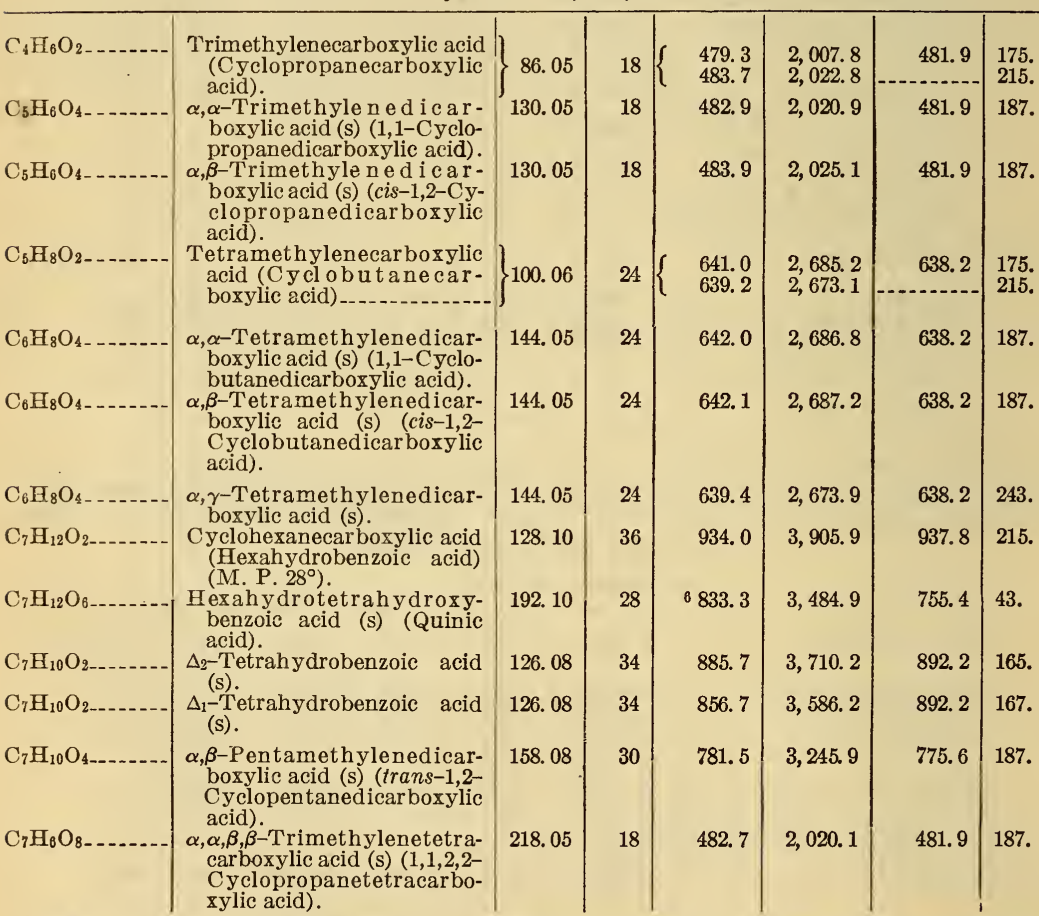

${ }^{6}$ The value for this compound appears to be entirely too large and would certainly bear reinvestigation. 


\section{TABLES OF DATA-Continued}

\section{CHO COMPOUNDS-Continued}

43. HYDROAROMATIC AND POLYMETHYLENE ACIDS-Continued

$Q=26.05 \times N+13 z+13 a a$

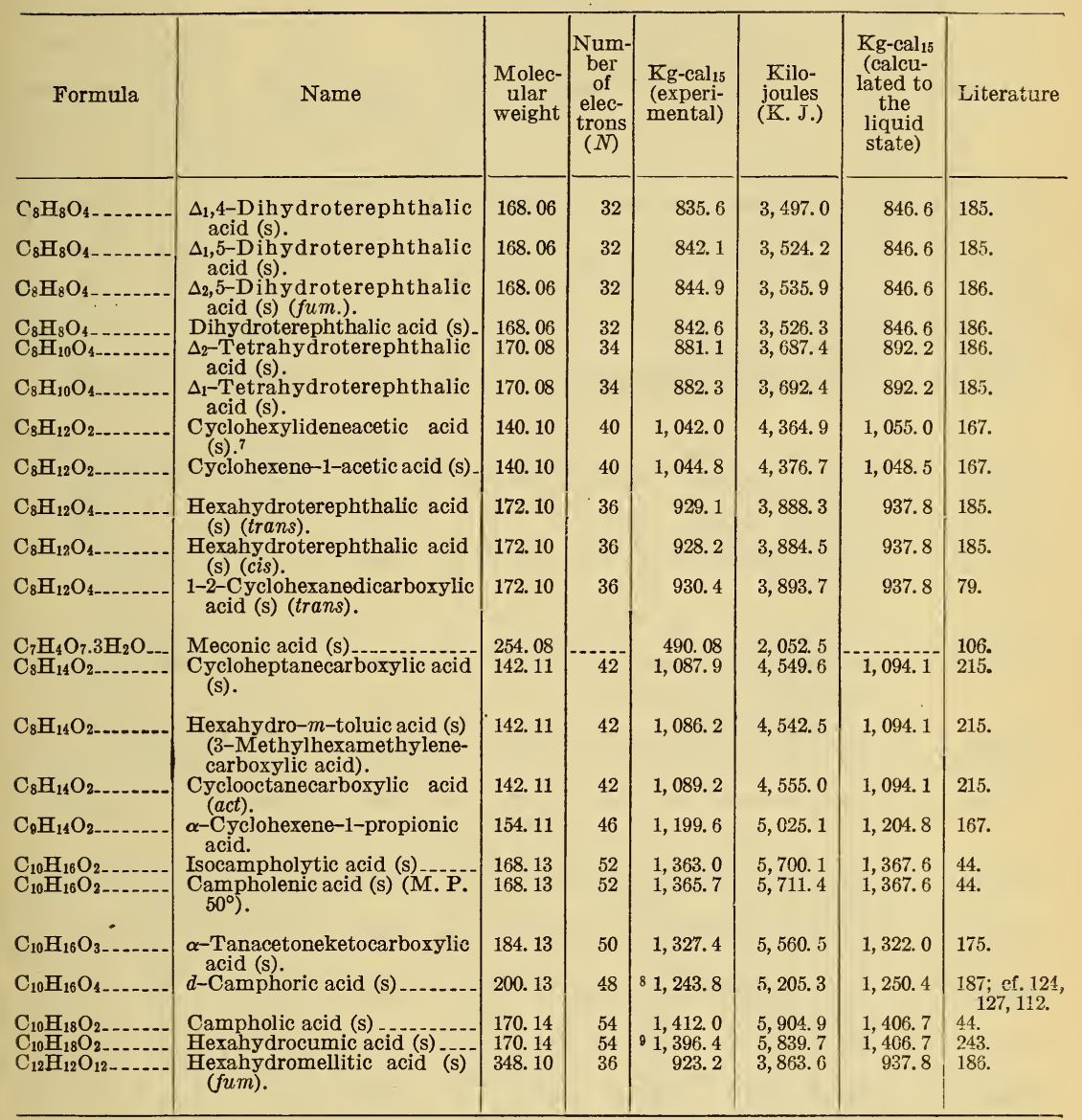

${ }_{7} \mathrm{C}_{6} \mathrm{H}_{10}$ : $\mathrm{CH} . \mathrm{COOH}$.

8 Values of (124), (127), and (112) vary between $1,242.5$ and $1,254.2 \mathrm{~kg}$-cal 15 .

- This value has been corrected according to the method employed by Swietoslawski, J. Amer. Chem. Soc., 42, p. 1093; 1920. The correction factor employed by him appears to be too large, however.

\section{ACID ANHYDRIDES}

$Q=26.05 \times N+10 \mathrm{bb}$

\begin{tabular}{|c|c|c|c|c|c|c|c|}
\hline $\mathrm{C}_{4} \mathrm{H}_{2} \mathrm{O}_{3} \ldots \ldots$ & Maleic anhydride (s) . . & 98.02 & 12 & 333.9 & $1,397.4$ & 339.1 & 182. \\
\hline $\mathrm{C}_{4} \mathrm{H}_{4} \mathrm{O}_{3} \ldots \ldots$ & Succinic anhydride (s) & 100.03 & 14 & 369. & $1,545.9$ & 374.3 & 182. \\
\hline $\mathrm{C}_{4} \mathrm{H}$ & $\left\{\begin{array}{l}\text { Acetic anhydride }(\nabla) \\
\text { Acetic anhydride (liq.) }\end{array}\right.$ & 102.00 & 16 & $\begin{array}{l}509.0 \\
458.3 \\
431.9\end{array}$ & $\begin{array}{l}1,540.8 \\
1,916.6 \\
1,806.2\end{array}$ & 426.8 & $\left\{\begin{array}{l}234 ; \text {, } 121 \\
223\end{array}\right.$ \\
\hline $\begin{array}{l}\mathrm{C}_{5} \mathrm{H}_{4} \mathrm{O}_{3} \ldots \\
\mathrm{C}_{5} \mathrm{H}_{6} \mathrm{O}_{3} \ldots \\
\mathrm{C}_{5} \mathrm{H}_{6} \mathrm{O}_{3} \ldots\end{array}$ & $\begin{array}{l}\text { Itaconic anhydride (s) } \\
\text { Glutaric anhydride (s) } \\
\text { Monomethylsuccinic anhy- } \\
\text { dride (s). }\end{array}$ & $\begin{array}{l}112.03 \\
114.05 \\
114.05\end{array}$ & $\begin{array}{l}18 \\
20 \\
20\end{array}$ & $\begin{array}{l}481.5 \\
528.0 \\
527.7\end{array}$ & $\begin{array}{l}2,015.1 \\
2,209.7 \\
2,208.4\end{array}$ & $\begin{array}{l}491.9 \\
531.0 \\
531.0\end{array}$ & $\begin{array}{l}183 . \\
183 . \\
234\end{array}$ \\
\hline
\end{tabular}




\section{TABLES OF DATA-Continued}

\section{CHO COMPOUNDS-Continued}

\section{ACID ANHYDRIDES-Continued}

$Q=26.05 \times N+10 \mathrm{bb}$

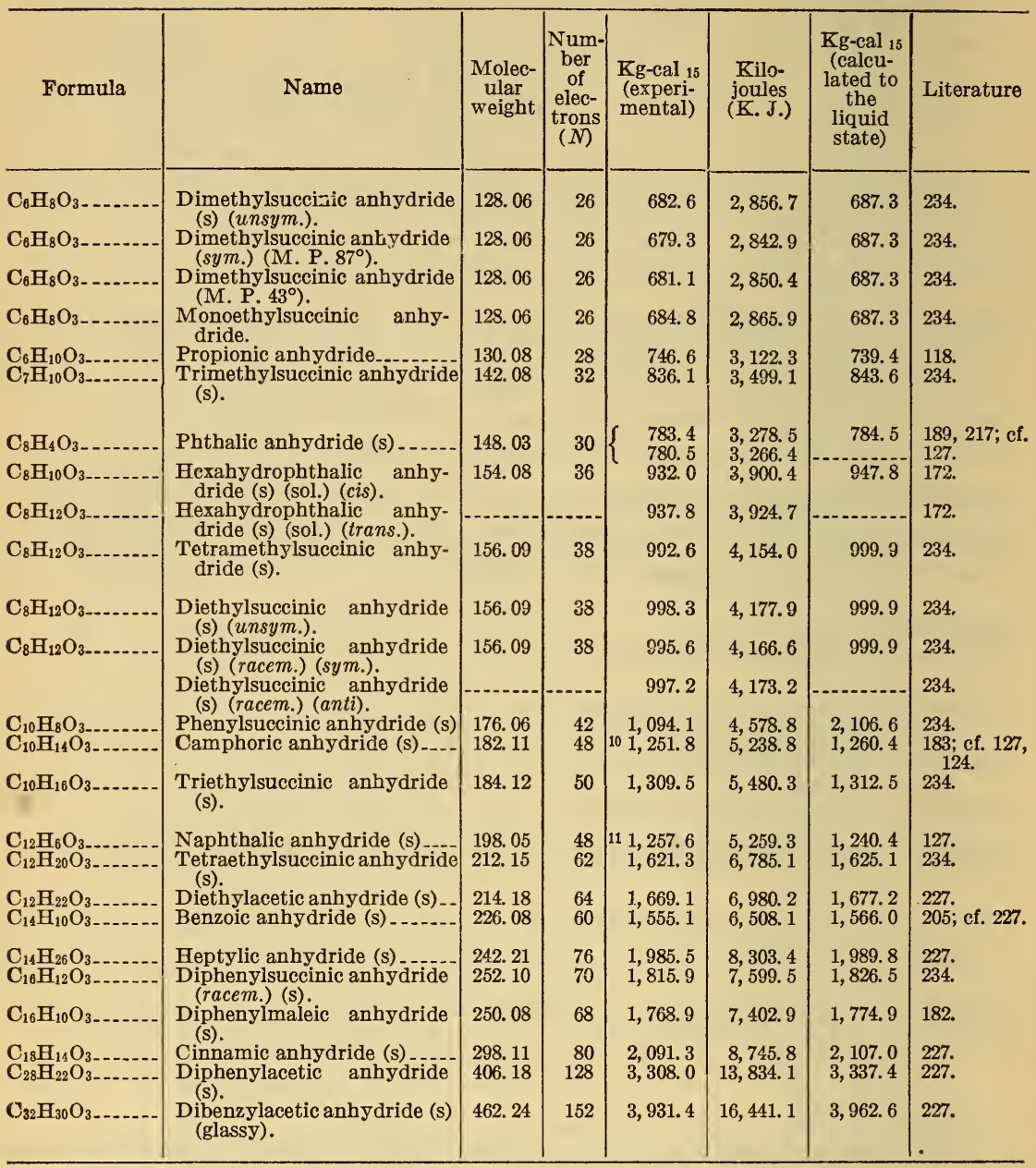

10 The mean of several values.

il Individual determinations do not agree better than 0.7 per cent; values of (127) are also on the whole 1 per cent higher than those of (183). 


\section{TABLES OF DATA-Continued}

\section{CHO COMPOUNDS-Continued}

45. LACTONES

$Q=26.05 \times N+13$

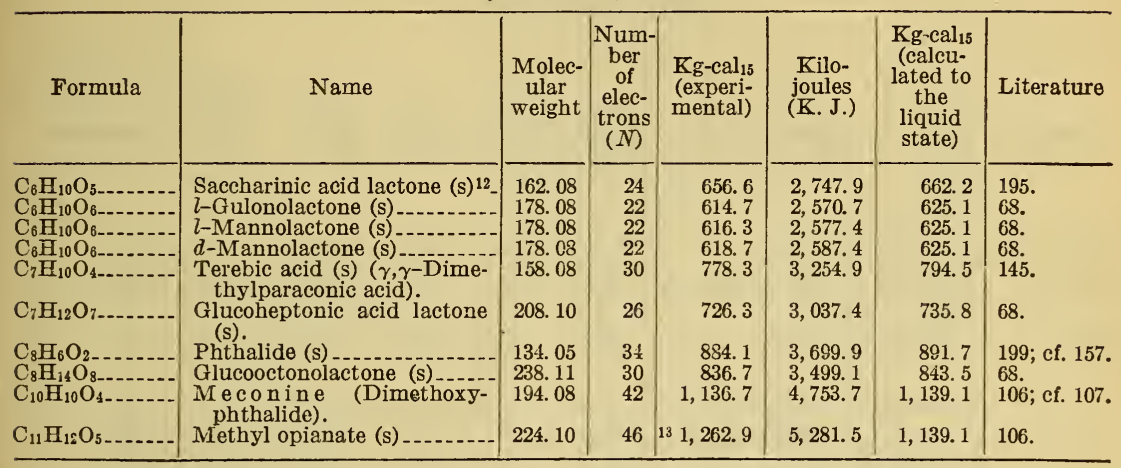

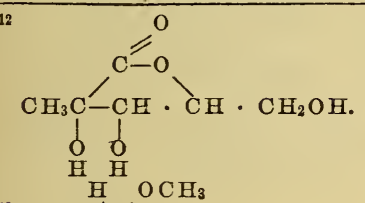

13

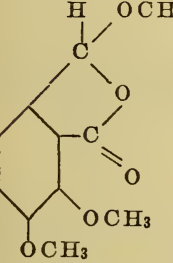

46. METHYL ESTERS OF MONOBASIC ACIDS

$Q=26.05 \times N+16.5$

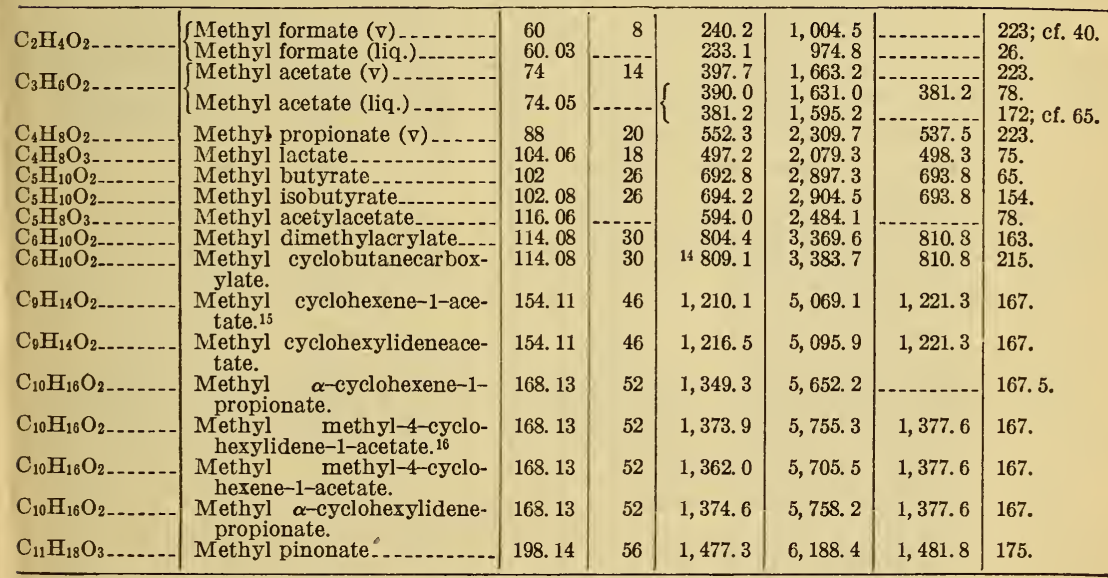

is Note correction for cyclobutane ring in formula for polymethylenecarboxylic acids.

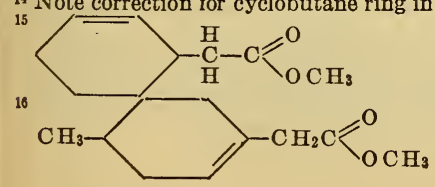




\section{TABLES OF DATA-Continued}

\section{CHO COMPOUNDS-Continued}

\section{METHYL ESTERS OF MONOBASIC AROMATIC ACIDS}

$Q=26.05 \times N+16.5-3.5 \mathrm{a}-6.5 \mathrm{~b}$

\begin{tabular}{|c|c|c|c|c|c|c|c|}
\hline Formula & Name & $\begin{array}{l}\text { Molec- } \\
\text { ular } \\
\text { weight }\end{array}$ & $\begin{array}{l}\text { Num- } \\
\text { ber } \\
\text { of } \\
\text { elec- } \\
\text { trons } \\
(N)\end{array}$ & $\begin{array}{l}\mathrm{Kg}_{\text {-cal }}{ }_{15} \\
\text { (experi- } \\
\text { mental) }\end{array}$ & $\begin{array}{c}\text { Kilo- } \\
\text { joules } \\
\text { (K. J.) }\end{array}$ & $\begin{array}{l}\mathrm{Kg}-\mathrm{cal}_{15} \\
\text { (calcu- } \\
\text { lated to } \\
\text { the } \\
\text { liquid } \\
\text { state) }\end{array}$ & Literature \\
\hline $\mathrm{C}_{2} \mathrm{H}_{8} \mathrm{O}_{2}$ & Methyl benzoate_. & 136. 06 & 36 & 943.5 & $3,948.6$ & 950.8 & 205. \\
\hline $\mathrm{C}_{8} \mathrm{H}_{8}$ & Methyl salicylate & 152.06 & 34 & 898.3 & $3,759.4$ & 902.2 & 206. \\
\hline $\mathrm{C}_{8} \mathrm{H}_{8} \mathrm{C}$ & Methyl $p$-hydroxybenzoate & 152. 06 & 34 & 895.4 & $3,747.3$ & 902.2 & 191. \\
\hline $\mathrm{C}_{8} \mathrm{H}_{5} \mathrm{O}_{5-}$ & Methyl gallate (s) - & 184. 06 & 30 & 801.6 & $3,354.7$ & 805.0 & 191. \\
\hline $\mathrm{C}_{9} \mathrm{H}_{10} \mathrm{O}_{3}$ & Methyl anisate (s) & 166. 08 & 40 & $1,068.7$ & $4,472.5$ & $1,074.5$ & 191. \\
\hline $\mathrm{C}_{10} \mathrm{H}_{10} \mathrm{O}_{2}$ & Methyl cinnamate (s) .... & 162. 08 & 46 & $\left\{\begin{array}{l}1,213.0 \\
1,210.4\end{array}\right.$ & $\begin{array}{l}5,076.4 \\
5,070.4\end{array}$ & $1,221.3$ & 191. \\
\hline $\mathrm{C}_{10} \mathrm{H}_{8} \mathrm{O}_{2-}$ & Methyl phenylpropiolate.- & 160.06 & 44 & $1,196.2$ & $5,002.5$ & $1,189.3$ & 138. \\
\hline $\mathrm{C}_{12} \mathrm{H}_{10} \mathrm{O}_{2}$ & Methyl $\beta$-naphthoate (s) & 186.08 & 54 & $1,401.5$ & $5,865.3$ & $1,406.7$ & 191. \\
\hline $\mathrm{C}_{12} \mathrm{H}_{14} \mathrm{O}_{3}$ & Methyl ethylcoumarate & 206.11 & 56 & $1,490.3$ & $6,242.9$ & $1,501.3$ & 177. \\
\hline $\mathrm{C}_{12} \mathrm{H}_{14} \mathrm{O}_{3-}$ & $\begin{array}{l}\text { Methyl ethylcoumarinate } \\
\text { (cis). }\end{array}$ & 206. 11 & 56 & $1,495.0$ & $6,262.6$ & $1,504.8$ & 177. \\
\hline
\end{tabular}

48. METHYL ESTERS OF POLYBASIC ALIPHATIC ACIDS

$Q=26.05 \times N+16.5 \mathrm{dd}$

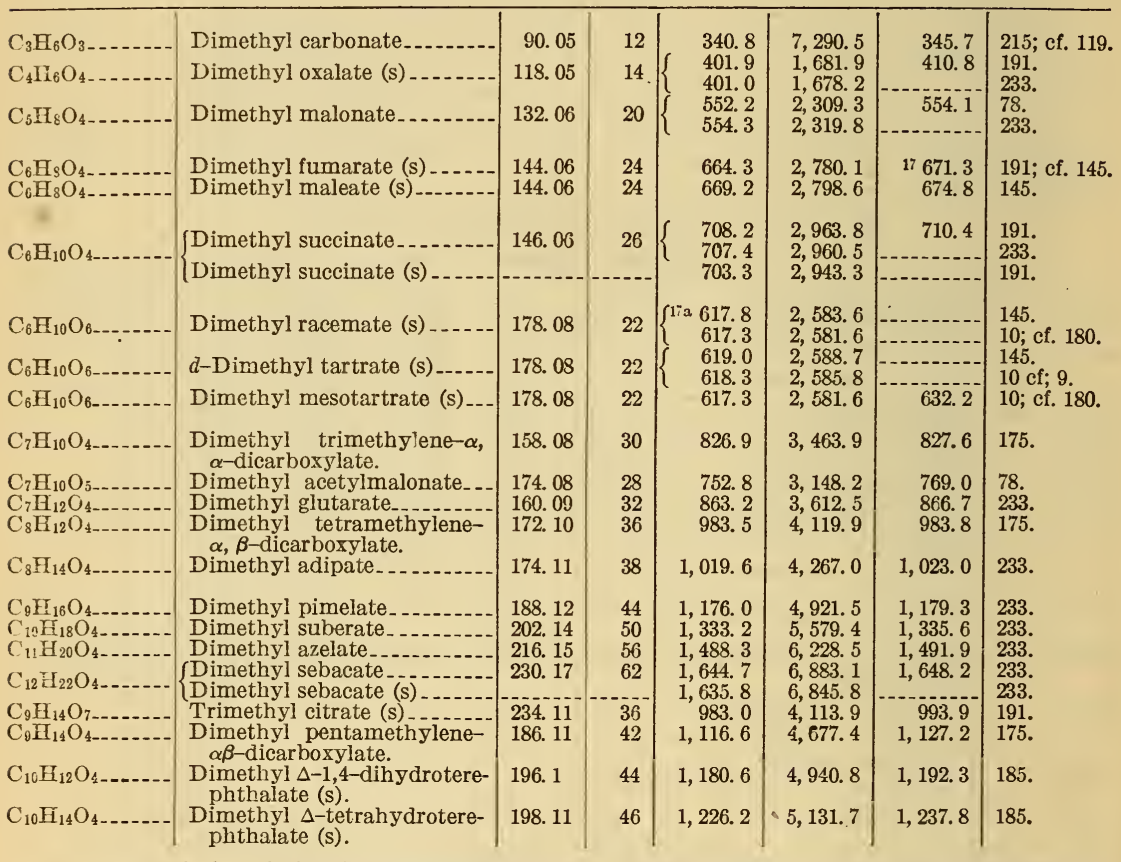

17 The heat of fusion of dimethyl fumarate is 8.3, which would bring the calculated value to approximately $663.0 \mathrm{~kg}$-cal.

1ia The work of (10) is much more accurate than that of (145), and preference should be given to his values. The values of (145) are included here merely to allow one to estimate the accuracy of his results and to use this information as a guide in estimating the accuracy of his measurements when no other measurements are available. His results vary widely. On the whole, they are not to be trusted to an accuracy greater than 0.5 per cent, and in many cases the values are not better than 1 to 2 per cent. 


\section{TABLES OF DATA-Continued}

\section{CHO COMPOUNDS-Continued}

48. METHYL ESTERS OF POLYBASIC ALIPHATIC ACIDS-Continued

$Q=26.05 \times N+16.5 \mathrm{dd}$

\begin{tabular}{|c|c|c|c|c|c|c|c|}
\hline Formula & Name & $\begin{array}{l}\text { Molec- } \\
\text { ular } \\
\text { weight }\end{array}$ & $\begin{array}{c}\text { Num- } \\
\text { ber } \\
\text { of } \\
\text { elec- } \\
\text { trons } \\
(N)\end{array}$ & $\begin{array}{l}\mathrm{Kg}_{\text {-cal }} \text { (5 } \\
\text { (experi- } \\
\text { mental) }\end{array}$ & $\begin{array}{l}\text { Kilo- } \\
\text { joules } \\
(\mathrm{K} . \mathrm{J} .)\end{array}$ & $\begin{array}{l}\mathrm{Kg}-\mathrm{cal}_{15} \\
\text { (calcu- } \\
\text { lated to } \\
\text { the } \\
\text { liquid } \\
\text { state) }\end{array}$ & Literature \\
\hline $\mathrm{C}_{10} \mathrm{H}_{14} \mathrm{O}_{8} \ldots \ldots$ & $\begin{array}{l}\text { Tetramethyl ethanetetracar- } \\
\text { boxylate (s) (sym.). }\end{array}$ & 262.11 & 38 & $1,045.1$ & $4,373.7$ & $1,056.0$ & 183. \\
\hline $\mathrm{C}_{10} \mathrm{H}_{16} \mathrm{O}_{4} \ldots \ldots$ & $\begin{array}{l}\text { Dimethyl norpinate (cis) } \\
\text { (Dimethyl 1,1-dimethyl- } \\
\text { cyclobutane-2,4-dicarbox- } \\
\text { ylate). }\end{array}$ & 200.13 & 48 & $\begin{array}{l}1,287.5 \\
-\end{array}$ & $5,393.3$ & $1,296.5$ & 175. \\
\hline $\mathrm{C}_{10} \mathrm{H}_{16} \mathrm{O}_{4} \ldots \ldots$ & $\begin{array}{l}\text { Dimethyl hexahydrotereph- } \\
\text { thalate (fum) (s) }\end{array}$ & 200.13 & 48 & $1,273.5$ & $5,329.6$ & $1,283.5$ & 185. \\
\hline $\mathrm{C}_{11} \mathrm{H}_{14} \mathrm{O}_{8} \ldots$ & $\begin{array}{l}\text { Tetramethyl } \alpha, \alpha, \beta, \beta \text {-tri- } \\
\text { methylenetetracarboxy- } \\
\text { late (s). }\end{array}$ & 274.11 & 42 & $1,169.7$ & $4,895.2$ & $1,173.2$ & 187. \\
\hline $\mathrm{C}_{11} \mathrm{H}_{16} \mathrm{O}_{4-}$ & $\begin{array}{l}\text { Dimethyl spiroheptanedi- } \\
\text { carboxylate. }\end{array}$ & 212.13 & 52 & $1,407.7$ & $5,896.9$ & $1,400.6$ & 175 . \\
\hline $\mathrm{C}_{11} \mathrm{H}_{10} \mathrm{O}_{8--}$ & $\begin{array}{l}\text { Tetramethyl methylenedi- } \\
\text { malonate } 18 \text { (s). }\end{array}$ & 276.13 & 44 & $1,201.4$ & $5,027.9$ & $1,212.3$ & 187. \\
\hline $\mathrm{C}_{11} \mathrm{H}_{18} \mathrm{O}_{4}$ & $\begin{array}{l}\text { Dimethyl } \alpha \text {-tanacetonedi- } \\
\text { carboxylate. }\end{array}$ & 214.14 & 54 & $1,451.4$ & $6,079.9$ & $1,452.7$ & 175. \\
\hline $\mathrm{C}_{11} \mathrm{H}_{18} \mathrm{O}_{4}$ & Dimethyl pinate. & 214. 14 & 54 & $1,440.2$ & $6,032.9$ & $1,452.7$ & 175. \\
\hline
\end{tabular}

${ }_{18}\left(\mathrm{CH}_{3} . \mathrm{CO}_{2}\right)_{2}$. $\mathrm{CH} . \mathrm{CH}_{2} \mathrm{CH}\left(\mathrm{CO}_{2} \mathrm{CH}_{3}\right)_{2}$.

49. METHYL ESTERS OF POLYBASIC AROMATIC ACIDS

$Q=26.05 \times N+16.5 \mathrm{dd}-3.5 \mathrm{a}-6.5 \mathrm{~b}$

\begin{tabular}{|c|c|c|c|c|c|c|c|}
\hline $\mathrm{C}_{10} \overline{\mathrm{H}}_{10} \mathrm{O}_{4 \ldots} \ldots$ & Dimethyl phthalato.... & 194.08 & 42 & $1,119.7$ & $4,685.9$ & $1,120.1$ & 191. \\
\hline $\mathrm{C}_{10} \mathrm{H}_{10} \mathrm{O}_{4} \ldots$ & Dimethyl isophthalate (s) & 194.08 & 42 & $1,111.1$ & $\overrightarrow{4}, 649.9$ & $1,120.1$ & 191. \\
\hline $\mathrm{C}_{10} \mathrm{H}_{10} \mathrm{O}_{4} \ldots \ldots$ & Dimethyl terephthalate (s) & 194.08 & 42 & $1,111.6$ & $4,652.0$ & $1,120.1$ & 191 ; cf. 185. \\
\hline $\mathrm{C}_{12} \mathrm{H}_{12} \mathrm{O}_{6} \ldots$ & Trimethyl trimesate (s) & 252.10 & 48 & $1,291.7$ & $5,405.8$ & $1,289.4$ & 191. \\
\hline $\mathrm{C}_{18} \mathrm{H}_{16} \mathrm{O}_{4} \ldots \ldots$ & $\begin{array}{l}\text { Dimethyl diphenylmaleate } \\
(\mathrm{s}) \text {. }\end{array}$ & 296.13 & 80 & $2,111.9$ & $8,838.3$ & $2,120.5$ & 183. \\
\hline $\mathrm{C}_{18} \mathrm{H}_{18} \mathrm{O}_{12} \ldots \ldots$ & Hexamethyl mellitate (s) & 426.13 & 66 & $191,824.3$ & $7,634.7$ & - & 191. \\
\hline $\mathrm{C}_{20} \mathrm{H}_{20} \mathrm{O}_{4} \ldots \ldots$ & Dimethyl $\beta$-truxillate (s) $\ldots .$. & 324.16 & $-\cdots$ & $2,421.6$ & $10,134.4$ & $---n---1$ & 183. \\
\hline
\end{tabular}

${ }^{19}$ This value is not quite satisfactory, although it is the mean of a number of determinations.

50. ETHYL ESTERS OF MONOBASIC ALIPHATIC ACIDS

$Q=25.05 \times N+16.5$

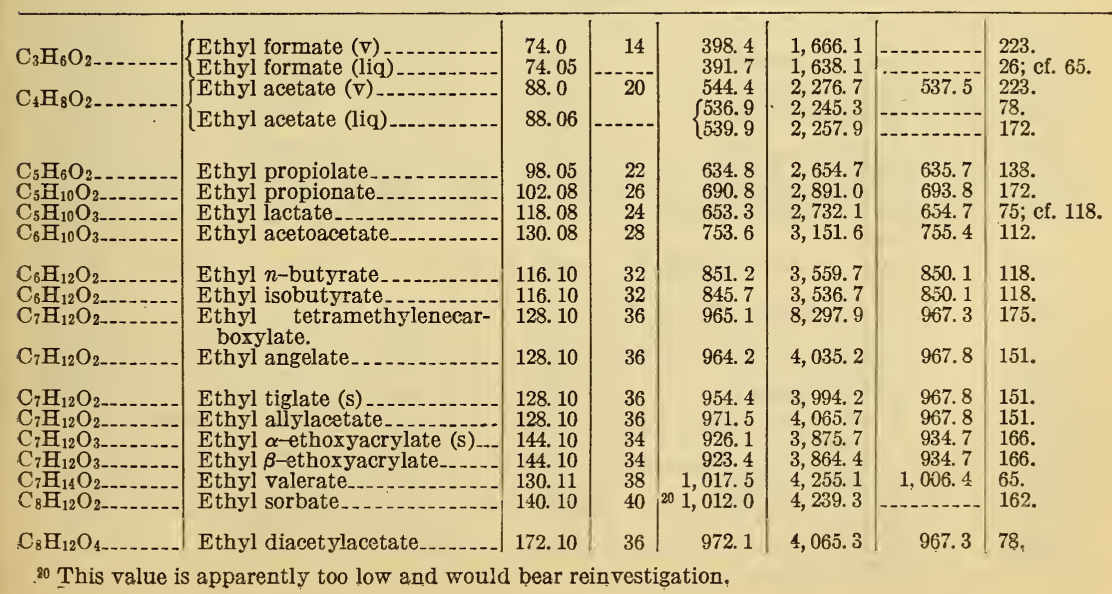




\section{TABLES OF DATA - Continued}

2. CHO COMPOUNDS-Continued

50. ETHYL ESTERS OF MONOBASIC ALIPHATIC ACIDS-Continued $Q=26.05 \times N+16.5$

\begin{tabular}{|c|c|c|c|c|c|c|c|}
\hline Formula & Name & $\begin{array}{l}\text { Molec- } \\
\text { ular } \\
\text { weight }\end{array}$ & \begin{tabular}{|c} 
Num- \\
ber \\
of \\
elec- \\
trons \\
$(N)$
\end{tabular} & $\begin{array}{l}\mathrm{Kg}_{\text {-cal }} \\
\text { (experi- } \\
\text { mental) }\end{array}$ & $\begin{array}{c}\text { Kilo- } \\
\text { joules } \\
\text { (K. J.) }\end{array}$ & $\begin{array}{c}\mathrm{Kg}-\mathrm{cal}_{15} \\
\text { (calcu- } \\
\text { lated to } \\
\text { the } \\
\text { liquid } \\
\text { state) }\end{array}$ & Literature \\
\hline $\mathrm{C}_{8} \mathrm{H}_{14} \mathrm{O}_{3}$ & Ethyl $\alpha$-ethoxycrotonate (s) & 158. 11 & 40 & 1, 076.9 & $4,506.8$ & $1,091.0$ & 166. \\
\hline $\mathrm{C}_{8} \mathrm{H}_{14} \mathrm{O}_{3}$ & Ethyl $\alpha$-ethylacetoacetate.-- & 158.11 & 40 & $1,070.9$ & $4,481.6$ & $1,068.0$ & 242. \\
\hline $\mathrm{C}_{10} \mathrm{H}_{16} \mathrm{O}_{2}$ & Ethyl $\beta, \delta$-dimethylsorbate & 168.13 & 52 & $211,351.3$ & $6,078.8$ & - - - - & 162. \\
\hline $\mathrm{C}_{10} \mathrm{H}_{10} \mathrm{O}_{2-}$ & Ethyl amylpropiolate & 168.13 & 52 & $1,394.0$ & $5,829.7$ & $1,404.2$ & 138. \\
\hline $\mathrm{C}_{10} \mathrm{H}_{16} \mathrm{O}_{2-}$ & $\begin{array}{l}\text { Et hy l (cyclohexene-1)-ace- } \\
\text { tate. }\end{array}$ & 168.13 & 52 & $1,360.4$ & $5,698.7$ & $1,377.6$ & 167. \\
\hline $\mathrm{C}_{10} \mathrm{H}_{10} \mathrm{O}_{2-}$ & $\begin{array}{l}\text { Ethylcyclohexylidene } \\
\text { acetate. }\end{array}$ & 168.13 & 52 & 1, 367. 0 & $5,726.4$ & $1,384.1$ & 167. \\
\hline $\mathrm{C}_{11} \mathrm{H}_{16} \mathrm{O}_{2-}$ & $\begin{array}{l}\text { Ethyl 1-methylcyclohexene- } \\
\text { 1-inethene-3-carboxylate. }\end{array}$ & 180.13 & & $1,477.0$ & $6,187.2$ & 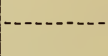 & 174. \\
\hline $\mathrm{C}_{11} \mathrm{H}_{18} \mathrm{O}_{2}$ & Ethyl hexylpropiolate....... & 182.14 & 58 & $1,550.8$ & $6,485.5$ & $1,560.5$ & 138. \\
\hline $\mathrm{C}_{11} \mathrm{H}_{18} \mathrm{O}$ & $\begin{array}{l}\text { E thy l } \alpha-(\text { cyclohexene-1) - } \\
\text { propionate. }\end{array}$ & 182.14 & 58 & ${ }^{22} 1,501.5$ & $6,289.8$ & & 167. \\
\hline $\mathrm{C}_{11} \mathrm{H}_{18} \mathrm{O}_{3}$ & $\begin{array}{l}\text { Ethyl methyl-1-ethyl-4-cy- } \\
\text { clopentanone carboxylate. } 23\end{array}$ & 198. 14 & & $1,465.8$ & $6,129.9$ & & 215. \\
\hline $\mathrm{C}_{12} \mathrm{H}_{18} \mathrm{O}_{2}$ & $\begin{array}{l}\text { E th y } 1 \text { 1,3-Dimethyl-4-cy- } \\
\text { clohexene-3-methene-5- } \\
\text { carboxylate. }\end{array}$ & 194.14 & & $1,631.4$ & $6,833.9$ & & 176. \\
\hline
\end{tabular}

21 This value appears to be low.

22 This value appears to be low.

${ }^{23}$ In the article by Swietoslawski (J. Amer. Chem. Soc., 40, p. 1099; 1920) the compound is named "ethyl ester of methylethyl-1,4-cyclopentanecarboxylic acid." However, neither the empirical formula nor the molecular weight corresponds to the above name. The above formula is suggested, for it corresponds to the molecular weight given.

\section{ETHYL ESTERS OF MONOBASIC AROMATIC ACIDS}

$Q=26.05 \times N+16.5-3.5 \mathrm{a}-6.5 \mathrm{~b}$

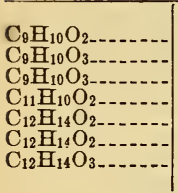

\begin{tabular}{l|l} 
Ethyl benzoate & 150.08 \\
Ethyl salicylate & 166.08 \\
Ethyl $p$-hydroxybenzoate (s) & 166.08 \\
Ethyl phenylpropiolate & 174.08 \\
Ethyl $\alpha$-methylcinnamate ${ }^{24}$ & 190.11 \\
Ethyl $\beta$-methylcinnamate_-- & 190.11 \\
Ethyl methylcoumarinate --- & 206.11
\end{tabular}

\begin{tabular}{l|l|}
42 & $1,098.7$ \\
40 & $1,051.2$ \\
40 & $1,042.8$ \\
50 & $1,338.6$ \\
58 & $1,526.8$ \\
58 & $1,527.5$ \\
56 & $1,494.2$
\end{tabular}

\begin{tabular}{|l|l|l|}
$4,598.1$ & $1,107.1$ & 205. \\
$4,399.3$ & $1,055.0$ & 206. \\
$4,364.1$ & $1,055.0$ & 206. \\
$5,598.0$ & $1,345.6$ & 138. \\
$6,395.8$ & $1,533.9$ & 163. \\
$6,398.7$ & $1,533.9$ & 163. \\
$6,259.2$ & $1,501.3$ & 177.
\end{tabular}

${ }^{24}$ See also Ber., 25, p. 90; 1892, for heats of combustion of polymers of ethyl cinnamate.

52. ETHYL ESTERS OF POLYBASIC ALIPHATIC ACIDS

$Q=26.05 \times N+16.5 \mathrm{dd}$

\begin{tabular}{|c|c|c|c|c|c|c|c|}
\hline $\mathrm{C}_{5} \mathrm{H}_{10} \mathrm{O}_{3} \ldots \ldots$ & Diethyl carbonate. & 118.08 & 24 & $\begin{array}{l}647.9 \\
642.1\end{array}$ & $\begin{array}{l}2,709.5 \\
2,685,3\end{array}$ & 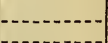 & $\begin{array}{l}215 . \\
119 .\end{array}$ \\
\hline $\begin{array}{l}\mathrm{C}_{6} \mathrm{H}_{10} \mathrm{O}_{4} \ldots \ldots \\
\mathrm{C}_{7} \mathrm{H}_{12} \mathrm{O}_{4} \ldots \ldots\end{array}$ & $\begin{array}{l}\text { Diethyl oxalate } \\
\text { Diethyl malonate............... }\end{array}$ & $\begin{array}{l}146.08 \\
160.10\end{array}$ & $\begin{array}{l}26 \\
32\end{array}$ & $\begin{array}{l}716.0 \\
860.4\end{array}$ & 2, 994. 3 & $\begin{array}{l}723.3 \\
866.6\end{array}$ & $\begin{array}{l}119 . \\
119 .\end{array}$ \\
\hline $\begin{array}{l}\mathrm{C}_{8} \mathrm{H}_{14} \mathrm{O}_{4} \\
\mathrm{C}_{8} \mathrm{H}_{14} \mathrm{O}_{6} \\
\mathrm{C}_{8} \mathrm{H}_{14} \mathrm{O}_{6} \\
\mathrm{C}_{8} \mathrm{H}_{10} \mathrm{O}_{4}\end{array}$ & $\begin{array}{l}\text { Diethyl succinate } \\
\text { Diethyl d-tartrate } \\
\text { Diethyl mesotartrate } \\
\text { Diethyl acetylenedicarboxy- } \\
\text { late. }\end{array}$ & $\begin{array}{l}\text { 174. } 11 \\
206.11 \\
206.11 \\
170.08\end{array}$ & $\begin{array}{l}38 \\
34 \\
34 \\
34\end{array}$ & $\begin{array}{r}1,007.3 \\
930.6 \\
931.3 \\
957.6\end{array}$ & $\begin{array}{l}4,212.5 \\
3,894.6 \\
3,896.9 \\
4,004.7\end{array}$ & $\begin{array}{r}1,022.9 \\
931.7 \\
931.7 \\
951.8\end{array}$ & $\begin{array}{l}119 . \\
9 . \\
9 . \\
138 .\end{array}$ \\
\hline $\mathrm{C}_{10} \mathrm{H}_{18} \mathrm{O}_{4-.}$ & Diethyl dimethylsuccinate & 202.14 & 50 & $1,328.9$ & $5,561.4$ & $1,335.5$ & 183. \\
\hline $\mathrm{C}_{10} \mathrm{H}_{18} \mathrm{O}_{4}$ & Diethyl dimethylsuccinate & 202. 14 & 50 & $1,322.9$ & $5,536.3$ & $1,335.5$ & 9. \\
\hline $\mathrm{C}_{10} \mathrm{H}_{18} \mathrm{O}_{4}$ & $\begin{array}{l}\text { Diethyl dimethylsuccinate } \\
\text { (meso) (s). }\end{array}$ & 202.14 & 50 & $1,324.1$ & $5,541.4$ & $1,335.5$ & 9. \\
\hline $\mathrm{C}_{12} \mathrm{H}_{20} \mathrm{O}_{7} \ldots \ldots$ & Triethyl citrate................. & 276.16 & 54 & $1,459.1$ & $6,101.9$ & $1,462.7$ & 118. \\
\hline $\mathrm{C}_{14} \mathrm{H}_{20} \mathrm{O}_{8} \ldots$ & $\begin{array}{l}\text { Tetraethyl ethylenetetracar- } \\
\text { boxylate (s) }\end{array}$ & 316.16 & 60 & $1,627.7$ & $6,811.9$ & $1,642.0$ & 183. \\
\hline $\mathrm{C}_{14} \mathrm{H}_{22} \mathrm{O}_{8} \ldots$ & $\begin{array}{l}\text { Tetraethyl ethanetetracar- } \\
\text { boxylate }(s y m .)(\mathrm{s})\end{array}$ & 318.18 & 62 & $1,661.2$ & $6,952.1$ & $1,681.1$ & \\
\hline $\mathrm{C}_{20} \mathrm{H}_{22} \mathrm{O}$ & $\begin{array}{l}\text { Diethyl diphenylsuccinate } \\
\text { (rac.) (s). }\end{array}$ & 326.18 & 94 & $2,450.8$ & $10,256.6$ & $2,474.9$ & 166. \\
\hline $\mathrm{C}_{20} \mathrm{H}_{22} \mathrm{O}_{4}$ & $\begin{array}{l}\text { Diethyl diphenylsuccinate } \\
\text { (meso) (s). }\end{array}$ & 326. 18 & 94 & $2,449.4$ & $10,250.7$ & $2,474.9$ & 166. \\
\hline
\end{tabular}




\section{TABLES OF DATA-Continued \\ 2. CHO COMPOUNDS-Continued}

\section{ESTERS OF ALIPHATIC ACIDS}

(All types)

$Q=26.05 \times N+16.5 \mathrm{dd}$

\begin{tabular}{|c|c|c|c|c|c|c|c|}
\hline Formula & Name & $\begin{array}{l}\text { Molec- } \\
\text { ular } \\
\text { weight }\end{array}$ & $\begin{array}{c}\text { Num- } \\
\text { ber } \\
\text { of } \\
\text { elec- } \\
\text { trons } \\
(N)\end{array}$ & $\begin{array}{l}\mathrm{Kg}-\mathrm{cal}_{15} \\
\text { (experi- } \\
\text { mental) }\end{array}$ & $\begin{array}{l}\text { Kilo- } \\
\text { joules } \\
\text { (K. J.) }\end{array}$ & $\begin{array}{c}\mathrm{Kg} \text {-cal }_{15} \\
\text { (calcu- } \\
\text { lated to } \\
\text { the } \\
\text { liquid } \\
\text { state) }\end{array}$ & Literature \\
\hline $\begin{array}{l}\mathrm{C}_{4} \mathrm{H}_{6} \mathrm{O}_{2} \\
\mathrm{C}_{4} \mathrm{H}_{6} \mathrm{O}_{2} \\
\mathrm{C}_{5} \mathrm{H}_{8} \mathrm{O}_{2} \\
\mathrm{C}_{7} \mathrm{H}_{14} \mathrm{O}_{2} \\
\mathrm{C}_{12} \mathrm{H}_{20} \mathrm{O}_{2} \\
\mathrm{C}_{18} \mathrm{H}_{36} \mathrm{O}_{2} \\
\mathrm{C}_{32} \mathrm{H}_{64} \mathrm{O}_{2}\end{array}$ & $\begin{array}{l}\text { Allyl formate (v) } \\
\text { Vinyl acetate } \\
\text { Allyl acetate } \\
\text { Amyl acetate. } \\
\text { Propyl hexylpropiolate. } \\
\text { Cetyl acetate (s) } \\
\text { Cetyl palmitate (s) }\end{array}$ & $\begin{array}{l}86.0 \\
86.05 \\
100.06 \\
130.11 \\
196.16 \\
284.2 \\
480.51\end{array}$ & $\begin{array}{r}18 \\
18 \\
24 \\
40 \\
64 \\
104 \\
188\end{array}$ & $\begin{array}{r}525.8 \\
498.1 \\
655.8 \\
251,042.5 \\
1,717.3 \\
2,728.1 \\
4,872.8\end{array}$ & $\begin{array}{r}2,198.9 \\
2,084.5 \\
2,742.6 \\
4,359.7 \\
7,181.8 \\
11,417.1 \\
20,392.7\end{array}$ & $\begin{array}{r}498.4 \\
654.7 \\
1,058.5 \\
1,716.8 \\
2,725.7 \\
4,913.9\end{array}$ & $\begin{array}{l}223 . \\
164 . \\
119 . \\
158 . \\
138 . \\
183 . \\
181 .\end{array}$ \\
\hline
\end{tabular}

25 The accuracy of the method employed and the value for the compound given are probably not better than 1 per cent.

\section{ESTERS OF AROMATIC ACIDS}

(All types)

$Q=26.05 \times N+16.5$

\begin{tabular}{|c|c|c|c|c|c|c|c|}
\hline $\mathrm{C}_{10} \mathrm{H}_{12} \mathrm{O}_{2}$ & Propyl benzoate & 164.10 & 48 & $1,254.5$ & $5,250.1$ & $1,263.4$ & 205. \\
\hline $\mathrm{C}_{10} \mathrm{H}_{12} \mathrm{O}_{3}-$ & Propyl salicylate & 180.10 & 46 & $1,205.7$ & $5,045.9$ & $1,214.8$ & 206. \\
\hline $\mathrm{C}_{10} \mathrm{H}_{12} \mathrm{O}_{3}$ & $\begin{array}{l}\text { Propyl } p \text {-hy d roxybenzo- } \\
\text { ate (s). }\end{array}$ & 180.10 & 46 & $1,200.6$ & $5,024.5$ & $1,214.8$ & 206. \\
\hline $\mathrm{C}_{11} \mathrm{H}_{14} \mathrm{O}_{2}$ & Isobutyl benzoate & 178.11 & 54 & $1,411.4$ & $5,906.7$ & $1,419.7$ & 205. \\
\hline $\mathrm{C}_{11} \mathrm{H}_{14} \mathrm{O}_{3}$ & Işobutyl salicylate.- & 194.0 & 52 & $1,365.5$ & $5,714.6$ & $1,371.1$ & 206. \\
\hline $\mathrm{C}_{12} \mathrm{H}_{16} \mathrm{O}_{2}$ & Amyl benzoate. & 192.13 & 60 & $1,569.5$ & $6,568.4$ & $1,576.0$ & 205. \\
\hline $\mathrm{C}_{12} \mathrm{H}_{14} \mathrm{O}_{2} \ldots$ & Propyl cinnamate & 190.11 & 58 & $1,526.0$ & $6,392.4$ & $1,533.9$ & 163. \\
\hline $\mathrm{C}_{48} \mathrm{H}_{38} \mathrm{O}_{12} \ldots \ldots$ & Mannitol hexabenzoate (s) & 806.30 & 206 & $5,358.5$ & $22,425.3$ & - & 206. \\
\hline
\end{tabular}

55. PHENOL ESTERS

$Q=26.05 \times N+16.5-3.5 \mathrm{a}-6.5 \mathrm{~b}+3.5 \mathrm{~m}$

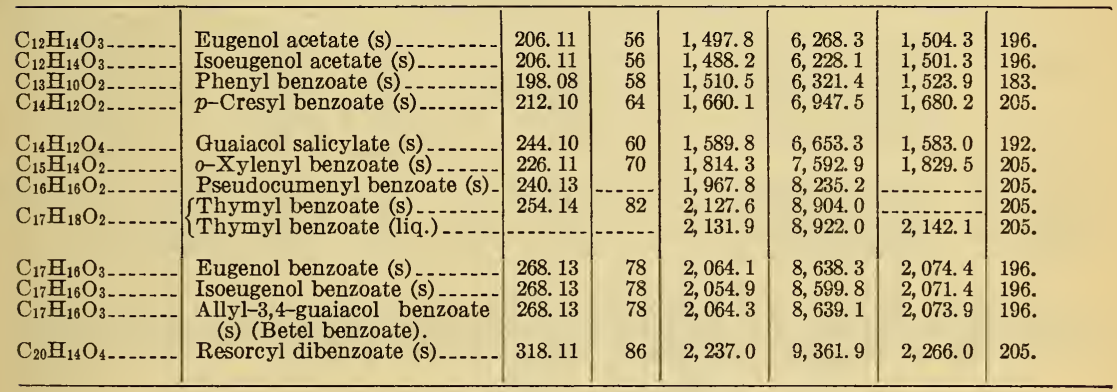

56. GLYCEROL ESTERS

\begin{tabular}{|c|c|c|c|c|c|c|c|}
\hline $\begin{array}{l}\mathrm{C}_{15} \mathrm{H}_{26} \mathrm{O}_{6} \ldots \ldots \\
\mathrm{C}_{15} \mathrm{H}_{20} \mathrm{O}_{6} \ldots \ldots \\
\mathrm{C}_{18} \mathrm{H}_{26} \mathrm{O}_{6} \ldots \ldots \\
\mathrm{C}_{24} \mathrm{H}_{20} \mathrm{O}_{6} \ldots \ldots \\
\mathrm{C}_{39} \mathrm{H}_{74} \mathrm{O}_{6} \ldots \ldots\end{array}$ & $\begin{array}{l}\text { Glyceryl tributyrate } \\
\text { Glyceryl tricyclobutyrate... } \\
\text { Glyceryl tricyclovalerate. } \\
\text { Glyceryl tribenzoate (s) } \\
\text { Glyceryl trilaurate (s) }\end{array}$ & $\begin{array}{l}302.21 \\
296.16 \\
338.21 \\
404.16 \\
638.59\end{array}$ & $\begin{array}{c}-- \\
--- \\
--\end{array}$ & $\begin{array}{l}1,941.1 \\
1,844.9 \\
2,308.6 \\
2,718.9 \\
5,706.3\end{array}$ & $\begin{array}{r}8,117.7 \\
7,715.4 \\
9,654.6 \\
11,378.6 \\
23,880.9\end{array}$ & 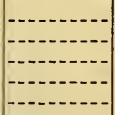 & $\begin{array}{l}215 . \\
215 . \\
215 . \\
206 \text {. } \\
193 ; \text { cf. } 120 .\end{array}$ \\
\hline $\begin{array}{l}\mathrm{C}_{45} \mathrm{H}_{86} \mathrm{O}_{6} \ldots \ldots \\
\mathrm{C}_{47} \mathrm{H}_{88} \mathrm{O}_{5} \ldots \ldots \\
\mathrm{C}_{47} \mathrm{H}_{88} \mathrm{O}_{5} \ldots \ldots \\
\mathrm{C}_{69} \mathrm{H}_{128} \mathrm{O}_{6} \ldots \ldots \\
\mathrm{C}_{68} \mathrm{E}_{128} \mathrm{O}_{6} \ldots \ldots\end{array}$ & $\begin{array}{l}\text { Glyceryl trimyristate } \\
\text { Glyceryl dibrassidate (s) } \\
\text { Glyceryl dierucate (s) } \\
\text { Glyceryl tribrassidate }(\mathrm{s}) \\
\text { Glyceryl trierucate (s) }\end{array}$ & $\begin{array}{l}722.69 \\
732.70 \\
732.70 \\
1,053.02 \\
1,053.02\end{array}$ & 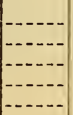 & $\begin{array}{r}6,650.3 \\
6,953.0 \\
6,979.0 \\
10,235.8 \\
10,264.7\end{array}$ & $\begin{array}{l}27,831.5 \\
29,098.3 \\
29,207.1 \\
42,836.8 \\
42,957.8\end{array}$ & 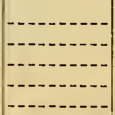 & $\begin{array}{l}193 ; \text { cf. } 120 . \\
193 . \\
193 . \\
193 . \\
193 .\end{array}$ \\
\hline
\end{tabular}




\section{TABLES OF DATA-Continued}

\section{IN COMPOUNDS}

\section{ALIPHATIC AMINES (PRIMARY)}

\section{$Q=26.05 \times N+13 \mathrm{ee}$}

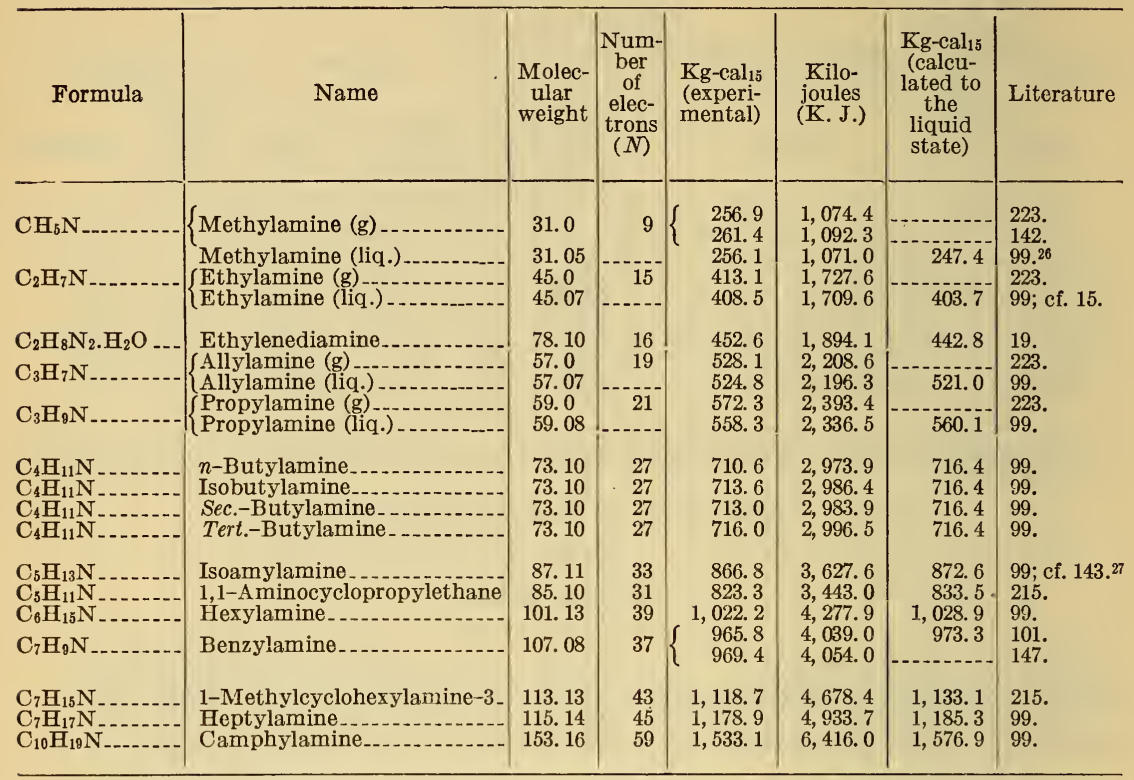

${ }_{26}$ The author (99) used naphthalene to standardize his bomb calorimeter. However, the value he used for naphthalene was $9,665.0 \mathrm{cal} . / \mathrm{g}$. The present best value is $9,617.0 \mathrm{cal} / \mathrm{g}$. His values, therefore, ought to be about 0.5 per cent too high, and they have accordingly been corrected by the proper factor. The data are probably not of a high degree of accuracy.

27 See footnote 28.

58. ALIPHATIC AMINES (SECONDARY)

$Q=26.05 \times N+19.5$

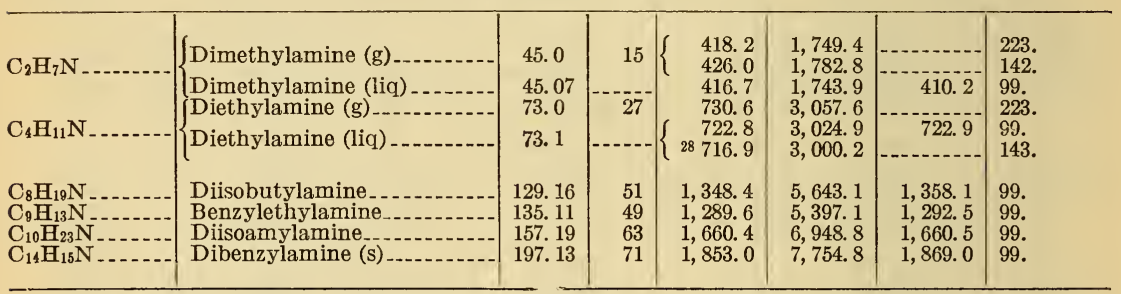

${ }_{28}$ Little significance should be attached to values of (143) for the redetermination of the heats of combustion of the three amines by the same author (142) some 25 years later showed enormous variations from the values obtained previously. The values by (142) are, on the whole, too low.

59. ALIPEATIC AMINES (TERTIARY)

$Q=26.05 \times N+26$

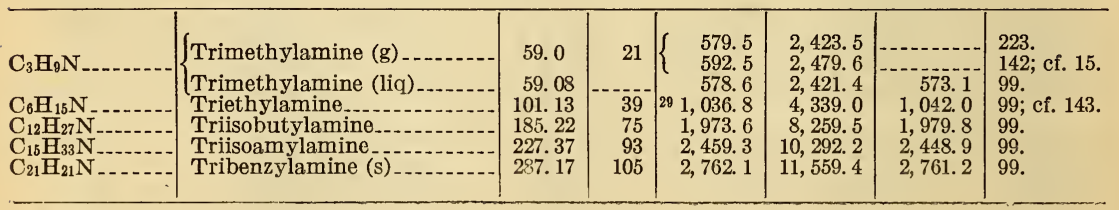

${ }^{20}$ See footnote 28 . 


\section{TABLES OF DATA-Continued}

\section{N COMPOUNDS-Continued.}

60. AROMATIC AMINES (PRIMARY)

$Q=26.05 \times N+6.5-3.5 \mathrm{hh}-3.5 \mathrm{a}-6.5 \mathrm{~b}$

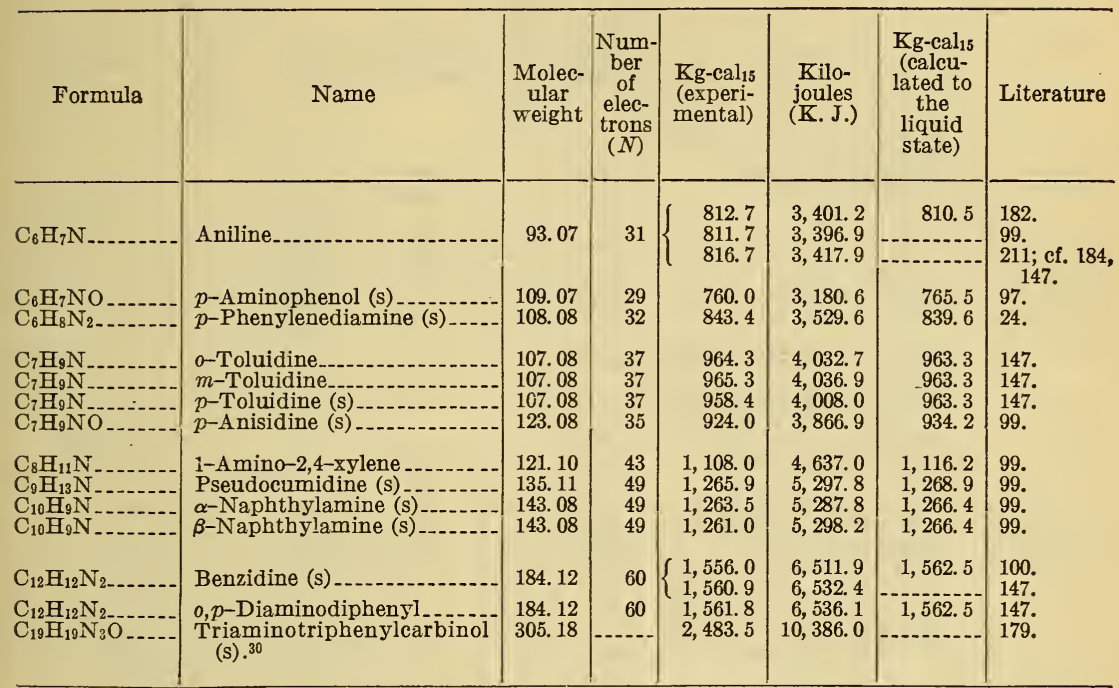

30 This article also contains the heats of combustion of rosaniline hydrochloride and the hexamethylrosaniline hydrochloride.

61. AROMATIC AMINES (SECONDARY)

$Q=26.05 \times N+13-3.5 \mathrm{hh}$

\begin{tabular}{|c|c|c|c|c|c|c|c|}
\hline $\mathrm{C}_{7} \mathrm{H}_{9} \mathrm{~N}_{11} \mathrm{~N} \ldots$ & $\begin{array}{l}\text { Methylaniline } \\
\text { Ethylaniline }\end{array}$ & $\begin{array}{l}107.08 \\
121.10\end{array}$ & $\begin{array}{l}37 \\
43\end{array}$ & $\begin{array}{r}973.5 \\
1,121.5\end{array}$ & $4,071.2$ & $\begin{array}{r}973.3 \\
1,129.6\end{array}$ & $147 ;$ cf. 72. \\
\hline $\mathrm{C}_{12} \mathrm{H}_{11} \mathrm{~N} \ldots \ldots$ & Diphenylamine (s) ........... & 169.10 & 59 & $\left\{\begin{array}{l}1,536.2 \\
1,530.2\end{array}\right.$ & $\begin{array}{l}6,428.9 \\
6,403.9\end{array}$ & $1,542.9$ & $\begin{array}{l}182 . \\
99 .\end{array}$ \\
\hline $\begin{array}{l}\mathrm{C}_{16} \mathrm{H}_{13} \mathrm{~N} \\
\mathrm{C}_{16} \mathrm{H}_{13} \mathrm{~N} \ldots \ldots\end{array}$ & $\begin{array}{l}\text { Phenyl- } \alpha \text {-naphthylamine (s) } \\
\text { Phenyl- } \beta \text {-naphthylamine (s) }\end{array}$ & $\begin{array}{l}\text { 219. } 11 \\
219.11\end{array}$ & $\begin{array}{l}77 \\
77\end{array}$ & $\begin{array}{l}2,003.8 \\
1,998.0\end{array}$ & $8,382.6$ & $\begin{array}{l}1,998.8 \\
1,998.8\end{array}$ & 99. \\
\hline
\end{tabular}

62. AROMATIC AMINES (TERTIARY)

$Q=26.05 \times N+19.5-3.5 \mathrm{hh}$

\begin{tabular}{|c|c|c|c|c|c|c|c|}
\hline $\begin{array}{l}\mathrm{C}_{3} \mathrm{H}_{11} \mathrm{~N} \\
\mathrm{C}_{10} \mathrm{H}_{15} \mathrm{~N} \\
\mathrm{C}_{18} \mathrm{H}_{15} \mathrm{~N}\end{array}$ & $\begin{array}{l}\text { Dimethylaniline } \\
\text { Diethylaniline } \\
\text { Triphenylamine (s) }\end{array}$ & $\begin{array}{l}121.10 \\
149.13 \\
245.13\end{array}$ & $\begin{array}{l}43 \\
55 \\
87\end{array}$ & $\begin{array}{l}1,142.7 \\
1,451.6 \\
2,267.8\end{array}$ & $\begin{array}{l}4,782.4 \\
6,074.9 \\
9,490.7\end{array}$ & $\begin{array}{l}1,136.1 \\
1,448.7 \\
2,275.3\end{array}$ & $\begin{array}{l}182 . \\
182 . \\
182 .\end{array}$ \\
\hline
\end{tabular}

63. AMINO ACIDS 31 (ALIPEATIC)

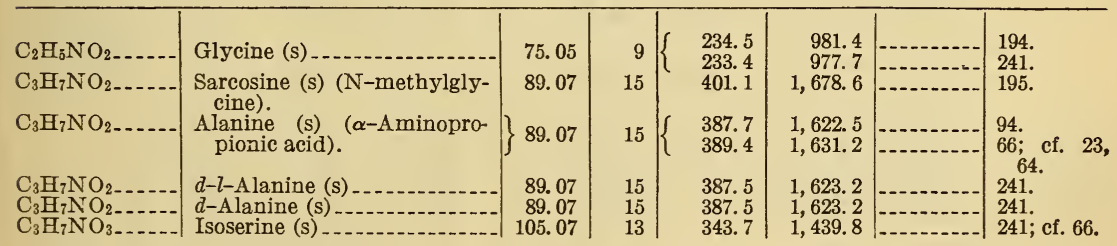

${ }^{31}$ The general expression $Q=26.05 \times N+a$ covers this class of compounds adequately. However, since no heat of fusion is available for this class, it is impossible to estimate the value of "a". "It is probably 6.5 . 


\section{TABLES OF DATA-Continued}

\section{N COMPOUNDS-Continued}

63. AMINO ACIDS (ALIPHATIC)-Continued

\begin{tabular}{|c|c|c|c|c|c|c|c|}
\hline Formula & Name & $\begin{array}{l}\text { Molec- } \\
\text { ular } \\
\text { weight }\end{array}$ & $\begin{array}{l}\text { Num- } \\
\text { ber } \\
\text { of } \\
\text { elec- } \\
\text { trons } \\
(N)\end{array}$ & $\begin{array}{l}\mathrm{Kg}-\mathrm{cal}_{15} \\
\text { (experi- } \\
\text { mental) }\end{array}$ & $\begin{array}{l}\text { Kilo- } \\
\text { joules } \\
\text { (K. J.) }\end{array}$ & $\begin{array}{l}\mathrm{Kg} \text { cal }_{15} \\
\text { (calcu- } \\
\text { lated to } \\
\text { the } \\
\text { liquid } \\
\text { state) }\end{array}$ & Literature \\
\hline $\mathrm{C}_{4} \mathrm{H}_{7} \mathrm{NO}_{4}$ & Diglycolamidic acid (s) & 133.07 & & 396.1 & $1,657.7$ & & 198. \\
\hline $\mathrm{C}_{4} \mathrm{H}_{7} \mathrm{NO}_{4}$ & Aspartic acid (s) & 133.07 & & 384.9 & $1,610.8$ & & $183 ;$ cf. 23. \\
\hline $\mathrm{C}_{4} \mathrm{H}_{7} \mathrm{NO}_{4--}$ & $l$-Aspartic acid (s) & 133.07 & & $\begin{array}{r}385.6 \\
32 \\
383.1\end{array}$ & $\begin{array}{l}1,615.3 \\
1,601.7\end{array}$ & & 66. \\
\hline $\mathrm{C}_{4} \mathrm{H}_{8} \mathrm{~N}_{2} \mathrm{O}_{3-}$ & Asparagine (s) & 132.08 & & 463. 3 & $1,938.9$ & & 194. \\
\hline $\mathrm{C}_{4} \mathrm{H}_{8} \mathrm{~N}_{2} \mathrm{O}_{3} \mathrm{H}_{2} \mathrm{O}$ & Asparagine (cryst) & 150. 10 & & 459. 7 & $1,922.0$ & & 64. \\
\hline $\mathrm{C}_{4} \mathrm{H}_{8} \mathrm{~N}_{2} \mathrm{O}_{3} \ldots$ & Glycylglycine (s) & 132.08 & & 470. 7 & $1,971.8$ & & 66. \\
\hline $\mathrm{C}_{5} \mathrm{H}_{8} \mathrm{~N}_{2} \mathrm{O}_{5}$ & Glycylglycinecarboxylic acid & 176.08 & & 471.3 & $1,974.3$ & & $241 ;$ cf. 66 \\
\hline $\mathrm{C}_{5} \mathrm{H}_{11} \mathrm{NO}_{2--}$ & dl- $\alpha$-A minoisovaleric acid (s) & 117. 10 & & 700.8 & $2,935.7$ & & 241. \\
\hline $\mathrm{C}_{5} \mathrm{H}_{9} \mathrm{NO}_{4-}$ & Glutamic acid (s) (act.) -- & 147.08 & & 542.4 & $2,272.1$ & & 66 ; cf. 64 \\
\hline $\mathrm{C}_{6} \mathrm{H}_{9} \mathrm{NO}_{6-}$ & Triglycolamidic acid (s) _. & 191. 0 & & 559.6 & $2,341.9$ & & 198. \\
\hline $\mathrm{C}_{6} \mathrm{H}_{10} \mathrm{~N}_{2} \mathrm{O}_{2}$ & Alanine anhydride $(\mathrm{s})^{\circ}$ & 142.10 & & 786.4 & $3,294.2$ & & 66. \\
\hline $\mathrm{C}_{6} \mathrm{H}_{10} \mathrm{~N}_{2} \mathrm{O}_{2} \ldots$ & $d$-Alanine anhydride (s) & 142. 10 & & 786.0 & $3,292.6$ & & 66. \\
\hline $\mathrm{C}_{6} \mathrm{H}_{11} \mathrm{~N}_{3} \mathrm{O}_{4}$ & Diglycylglycine (s) & 189. 12 & -- & 709.8 & $2,973.4$ & & 241. \\
\hline $\mathrm{C}_{0} \mathrm{H}_{12} \mathrm{~N}_{2} \mathrm{O}_{3} \ldots$ & Glycylglycine ethyl ester (s) & 160.12 & & 803. 2 & $3,364.6$ & & 66. \\
\hline $\mathrm{C}_{6} \mathrm{H}_{13} \mathrm{NO}_{2-}$ & $\begin{array}{l}\text { Leucine (s) ( } \alpha \text {-aminoisocap- } \\
\text { roic acid). }\end{array}$ & 131. 11 & & 855.6 & $3,580.7$ & & $\begin{array}{l}194 \text {; cf. } 66 \text {, } \\
23 \text {. }\end{array}$ \\
\hline $\mathrm{C}_{7} \mathrm{H}_{13} \mathrm{NO}_{3-.}$ & Formyl-d, $j$-leucine (s) & 159. 11 & & 917.4 & $3,842.9$ & & 241. \\
\hline $\mathrm{C}_{8} \mathrm{H}_{16} \mathrm{~N}_{2} \mathrm{O}_{3} \ldots$ & $d$-l-Leucylglycine $(\mathrm{s})$ & 188. 15 & & $1,095.3$ & $4,588.2$ & & 241. \\
\hline $\mathrm{C}_{8} \mathrm{H}_{14} \mathrm{~N}_{4} \mathrm{O}_{5}$ & Triglycylglycine (s) & 246.15 & & 946.6 & $3,965.3$ & & 241. \\
\hline $\mathrm{C}_{9} \mathrm{H}_{16} \mathrm{~N}_{2} \mathrm{O}_{5}$ & $\alpha$-Carbethoxyglycylglycine & 232.15 & & $1,120.4$ & $4,693.4$ & & 66. \\
\hline $\mathrm{C}_{9} \mathrm{H}_{16} \mathrm{~N}_{2} \mathrm{C}$ & $\begin{array}{l}\beta \text {-Carbethoxyglycylglycine } \\
\text { ethyl ester (s). }\end{array}$ & 232.15 & & $1,091.9$ & $4,573.9$ & & 66. \\
\hline $\mathrm{C}_{10} \mathrm{H}_{19} \mathrm{~N}_{3} \mathrm{O}_{4} \ldots$ & Leucylglycylglycine (s) & 245. 18 & & $1,333.1$ & $5,584.4$ & & 66. \\
\hline $\mathrm{C}_{12} \mathrm{H}_{22} \mathrm{~N}_{2} \mathrm{O}_{2}$ & $\begin{array}{l}\text { Leucineimide (s) (3,6-diiso- } \\
\text { butyl-2,5-diacipiperazine). }\end{array}$ & 226. 20 & & $1,723.8$ & $7,220.9$ & & 66. \\
\hline
\end{tabular}

32 See footnote $74 b$.

64. AMINO ACIDS

(Containing a phenyl radical)

\begin{tabular}{|c|c|c|c|c|c|c|c|}
\hline $\begin{array}{l}\mathrm{C}_{8} \mathrm{H}_{9} \mathrm{NO}_{2} \ldots \\
\mathrm{C}_{8} \mathrm{H}_{9} \mathrm{NO}_{2} \ldots \\
\mathrm{C}_{9} \mathrm{H}_{11} \mathrm{NO}_{2} \ldots \\
\mathrm{C}_{9} \mathrm{H}_{11} \mathrm{NO}_{3} \ldots \ldots\end{array}$ & $\begin{array}{l}\text { Phenylglycine (s) } \\
\text { Anilidoacetic acid }(\mathrm{s}) \\
\text { Phenylalanine (s) } \\
\text { Tyrosine (s) }\end{array}$ & $\begin{array}{l}\text { 151. } 08 \\
151.08 \\
165.10 \\
181.10\end{array}$ & 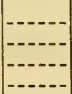 & $\begin{array}{r}955.1 \\
965.2 \\
1,111.3 \\
1,070.2\end{array}$ & $\begin{array}{l}4,000.9 \\
4,043.2 \\
4,655.2 \\
4,474.5\end{array}$ & 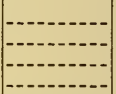 & $\begin{array}{l}66 . \\
66 . \\
66 . \\
64 \text {; cf. } 23 .\end{array}$ \\
\hline
\end{tabular}

65. AMIDES (ALIPHATIC)

$Q=20.05 \times N$

\begin{tabular}{|c|c|c|c|c|c|c|c|}
\hline $\mathrm{H}_{3} \mathrm{~N}$ & Formamide. & 45. 03 & 5 & 134.9 & 564.6 & & 209. \\
\hline $\mathrm{CH}_{4} \mathrm{~N}_{2} \mathrm{O}_{-}$ & Urea (s) & 60.05 & 6 & 151. 6 & 633.8 & 156.3 & 64; cf. 194, \\
\hline $\mathrm{C}_{2} \mathrm{H}_{3} \mathrm{NO}$ & Oxamic acid (s) & 89.03 & 5 & $\begin{array}{l}\text { 128. } 6 \\
132.6\end{array}$ & $\begin{array}{l}537.8 \\
554.9\end{array}$ & 130.2 & $\begin{array}{l}130 .{ }^{33} \\
184 .\end{array}$ \\
\hline $\mathrm{C}_{2} \mathrm{H}_{4} \mathrm{~N}_{2} \mathrm{O}_{2}$ & Oxamide (s) - & 88. 05 & 8 & 203.2 & 850.4 & 208.4 & 184. \\
\hline $\mathrm{C}_{2} \mathrm{H}_{4} \mathrm{~N}_{2} \mathrm{O}_{2}$ & Formylurea & 88.05 & & 207.0 & 865. 7 & & 130. \\
\hline $\mathrm{C}_{2} \mathrm{H}_{5} \mathrm{NO} \ldots$ & A cetamide (s) & 59.05 & 11 & 282.6 & $1,182.7$ & 286.5 & 209. \\
\hline $\mathrm{C}_{3} \mathrm{H}_{4} \mathrm{~N}_{2} \mathrm{O}_{4}$ & Oxaluric acid (s) & 132. 05 & 8 & 207.5 & 868.4 & 208. 0 & 130. \\
\hline $\mathrm{C}_{3} \overline{\mathrm{H}}_{5} \mathrm{NO}_{3}$ & Methyl oxamate (s) & 103. 05 & 11 & 305.4 & $1,278.1$ & 303. 0 & 184. \\
\hline $\mathrm{C}_{3} \mathrm{H}_{6} \mathrm{~N}_{2} \mathrm{O}_{2} \ldots$ & Malonamide (s) & 102.07 & 14 & 358.8 & $1,501.6$ & 364.7 & 184. \\
\hline $\mathrm{C}_{3} \mathrm{H}_{6} \mathrm{~N}$ & Acetylurea (s) & 102.07 & 14 & 360.9 & $1,510.4$ & 364.7 & 130. \\
\hline $\mathrm{C}_{3} \mathrm{H}_{6} \mathrm{~N}_{2} \mathrm{O}$ & Hydantoic acid (s) & 118.07 & 12 & 308. 6 & $1,291.5$ & 312. 6 & 130. \\
\hline $\mathrm{C}_{3} \mathrm{H}_{7} \mathrm{NO}$ & Propionamide (s) & 105. 07 & 17 & 439. 9 & $1,840.9$ & 442.8 & 209. \\
\hline $\mathrm{C}_{3} \mathrm{H}_{7} \mathrm{NO}$ & Urethane (s) & 89.07 & 15 & 397.2 & $1,662.3$ & 402.7 & 184. \\
\hline $\mathrm{C}_{3} \mathrm{H}_{8} \mathrm{~N}_{2} \mathrm{O}$ & Ethylurea (s) & 88. $\theta 8$ & 18 & 472.0 & $1,975.3$ & 475.4 & 130. \\
\hline
\end{tabular}

${ }_{33}$ The author (130.5) gives the heat of combustion of guanidine nitrate, $\mathrm{CH}_{6} \mathrm{~N}_{4} \mathrm{O}_{3}$. 


\section{TABLES OF DATA-Continued}

\section{N COMPOUNDS-Continued}

65. AMIDES (ALIPHATIC)-Continued

$Q=26.05 \times N$

\begin{tabular}{|c|c|c|c|c|c|c|c|}
\hline Formula & Name & $\begin{array}{c}\text { Molec- } \\
\text { ular } \\
\text { weight }\end{array}$ & $\begin{array}{c}\text { Num- } \\
\text { ber } \\
\text { of } \\
\text { elec- } \\
\text { trons } \\
(N)\end{array}$ & $\begin{array}{l}\mathrm{Kg}_{\text {-cal }}{ }_{15} \\
\text { (experi- } \\
\text { mental) }\end{array}$ & $\begin{array}{c}\text { Kilo- } \\
\text { joules } \\
\text { (K. J.) }\end{array}$ & $\begin{array}{l}\mathrm{Kg}_{\mathrm{g}-\mathrm{cal}} 15 \\
\text { (calcu- } \\
\text { lated to } \\
\text { the } \\
\text { liquid } \\
\text { state) }\end{array}$ & Literature \\
\hline $\mathrm{C}_{4} \mathrm{H}_{7} \mathrm{NO}_{3}$ & Ethyl oxamate (s) & 117.07 & 17 & 457.3 & $1,913.8$ & 459.3 & 184. \\
\hline $\mathrm{C}_{4} \mathrm{H}_{7} \mathrm{~N}_{3} \mathrm{O}$ & Creatinine (s) - & 113.08 & & 503.4 & $2,355.6$ & & \\
\hline $\mathrm{C}_{4} \mathrm{H}_{8} \mathrm{~N}_{2} \mathrm{O}$ & Succinamide (s) & 116.08 & 20 & 509.2 & $2,131.0$ & 521.0 & 184. \\
\hline $\mathrm{C}_{4} \mathrm{H}_{8} \mathrm{~N}_{2} \mathrm{O}$ & d-Tartramide & 148. 08 & 16 & 427.0 & $1,786.9$ & 429.8 & 54. \\
\hline $\mathrm{C}_{4} \mathrm{H}_{8} \mathrm{~N}_{2} \mathrm{O}$ & Mesotartramide. & 148. 08 & 16 & 426. 6 & $1,785.3$ & 429.8 & 54. \\
\hline $\mathrm{C}_{4} \mathrm{H}_{9} \mathrm{NO}$ & n-Butyramide (s) & 87. 08 & 23 & 596.0 & $2,494.3$ & 599. 2 & 209. \\
\hline $\mathrm{C}_{4} \mathrm{H}_{9} \mathrm{~N}$ & Isobutyramide (s) & 87.08 & 23 & 595.9 & $2,493.8$ & 599.2 & 209. \\
\hline $\mathrm{C}_{4} \mathrm{H}_{2} \mathrm{~N}_{3} \mathrm{O}_{2}$ & Creatine (anhydr.) (s) & 131.10 & & $\begin{array}{l}559.8 \\
555.4\end{array}$ & $\begin{array}{l}2,342.8 \\
2,322.1\end{array}$ & & $\begin{array}{l}194 . \\
64 .\end{array}$ \\
\hline $\mathrm{C}_{4} \mathrm{H}_{9} \mathrm{~N}_{3} \mathrm{O}_{2} \cdot \mathrm{H}_{2}$ & Creatine (cryst) & 149. 12 & & 553.1 & $2,314.7$ & & 194. \\
\hline $\mathrm{C}_{5} \mathrm{H}_{10} \mathrm{~N}_{2} \mathrm{O}_{2}$ & Dimethylmalonamide (sym.) & 130.1 & 26 & 685.8 & $2,870.1$ & 677.3 & 184. \\
\hline $\mathrm{C}_{5} \mathrm{H}_{11} \mathrm{NO}$ & Isovaleramide (s) & 101. 10 & 29 & 751.5 & $3,145.0$ & 755.5 & 209. \\
\hline $\mathrm{C}_{7} \overline{\mathrm{H}}$ & Diethylmalonamide (s) & 158.12 & 38 & 994.8 & $4,163.2$ & 989.9 & 184. \\
\hline $\mathrm{C}_{8} \mathrm{H}_{1}$ & Amylpropiolamide (s) & 139.11 & 43 & $1,150.4$ & $4,810.9$ & $1,153.2$ & 138. \\
\hline $\mathrm{C}_{8} \mathrm{H}_{16} \mathrm{~N}_{2} \mathrm{O}_{4}$ & $d-$ Tartaricdiethylamide & 204. 14 & 40 & $1,064.1$ & $4,453.3$ & $1,068.0$ & 54. \\
\hline $\mathrm{C}_{8} \mathrm{H}_{16} \mathrm{~N}_{2} \mathrm{O}$ & $d l$-Tartaricdiethylamide & 204.14 & 40 & $1,064.3$ & $4,454.1$ & $1,068.0$ & 54. \\
\hline $\mathrm{C}_{8} \mathrm{H}_{10} \mathrm{~N}_{2} \mathrm{C}$ & Mesotartaricdiethylamide & 204.14 & 40 & 1, 065.3 & $4,458.3$ & $1,068.0$ & 54. \\
\hline $\mathrm{C}_{9} \overline{\mathrm{H}}_{15} \mathrm{NC}$ & Hexylpropiolamide (s) & 153.13 & 49 & $1,308.0$ & $5,470.1$ & $1,309.5$ & 138. \\
\hline
\end{tabular}

66. ACID AMIDES (AROMATIC)

$Q=26.05 \times N-3.5 \mathrm{hh}$

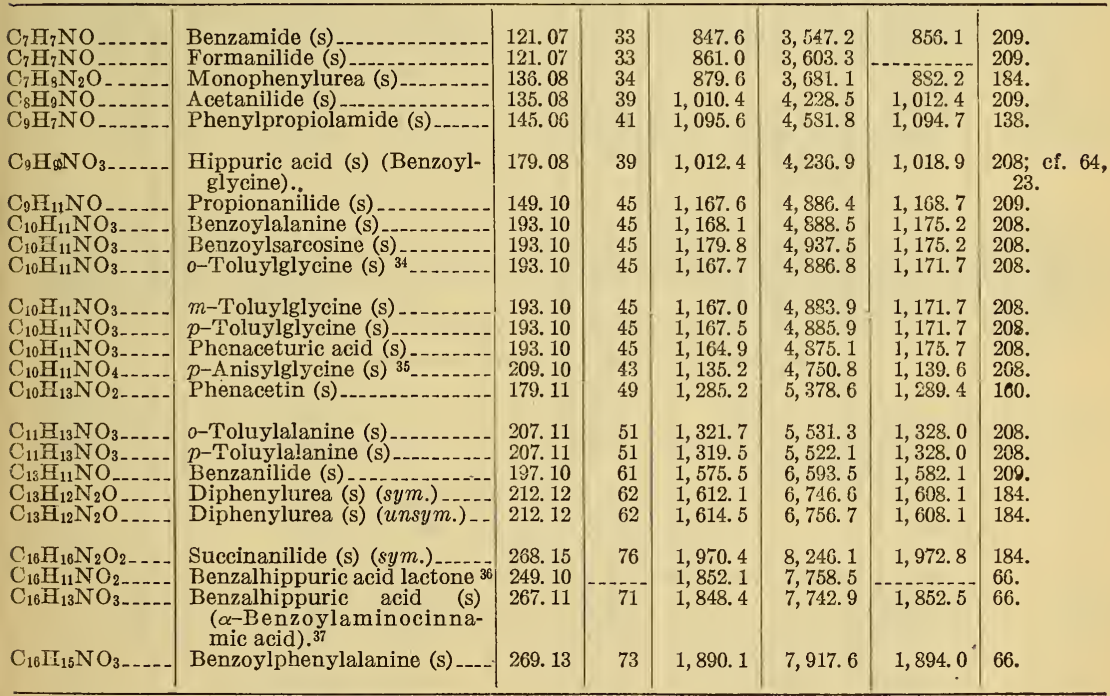

\footnotetext{
${ }_{34} \mathrm{CH}_{3} \cdot \mathrm{C}_{6} \mathrm{H}_{4} \cdot \mathrm{CONH} . \mathrm{CH}_{2}$. $\mathrm{COOH}$.

${ }_{35} \mathrm{H}_{3} \mathrm{CO} . \mathrm{C}_{6} \mathrm{H}_{4}$. CO.NH. $\mathrm{CH}_{2} \mathrm{CO}_{2} \mathrm{H}$.

${ }_{36} \mathrm{C}_{6} \mathrm{H}_{5} \mathrm{CO} . \mathrm{N}-\mathrm{C}=\mathrm{CH} . \mathrm{C}_{6} \mathrm{H}_{5}$.<smiles>[CH][Co]</smiles>

${ }^{37} \mathrm{C}_{6} \mathrm{H}_{5} \mathrm{CH}: \mathrm{C}\left(\mathrm{CO}_{2} \mathrm{H}\right) \cdot \mathrm{HN} . \mathrm{OC} . \mathrm{C}_{6} \mathrm{H}_{8}$. $3697^{\circ}-29-4$
} 


\section{TABLES OF DATA-Continued}

\section{IN COMPOUNDS-Continued}

67. CYCLIC UREIDES, HYDANTOINS, PYRIMIDINES, PURINES

\begin{tabular}{|c|c|c|c|c|c|c|c|}
\hline Formula & Name & $\begin{array}{l}\text { Molec- } \\
\text { ular } \\
\text { weight }\end{array}$ & $\begin{array}{l}\text { Num- } \\
\text { ber } \\
\text { of } \\
\text { elec- } \\
\text { trons } \\
(N)\end{array}$ & $\begin{array}{l}\mathrm{Kg}^{-\mathrm{cal}_{15}} \\
\text { (experi- } \\
\text { mental) }\end{array}$ & $\begin{array}{c}\text { Kilo- } \\
\text { joules } \\
\text { (K. J.) }\end{array}$ & $\begin{array}{l}\mathrm{Kg} \text {-cal } 15 . \\
\text { (calcu- } \\
\text { lated to } \\
\text { the } \\
\text { liquid } \\
\text { state) }\end{array}$ & Literature \\
\hline $\mathrm{C}_{3} \mathrm{H}_{2} \mathrm{~N}_{2} \mathrm{O}_{3-}$ & Parabanic acid (s) l(Oxayl- & 114.04 & & 212.4 & 888.9 & & 130. \\
\hline $\mathrm{C}_{3} \mathrm{H}_{4} \mathrm{~N}_{2} \mathrm{O}$ & $\begin{array}{l}\text { urea) } \\
\text { Hydantoin (s) }\end{array}$ & 100.05 & & 311.7 & $1,304.5$ & & 130. \\
\hline $\mathrm{C}_{4} \mathrm{H}_{2} \mathrm{~N}_{2} \mathrm{O}$ & Alloxan (s) & 160. 05 & & 276.3 & $1,156.3$ & & 130. \\
\hline $\mathrm{C}_{4} \mathrm{H}_{5} \mathrm{~N}_{3} \mathrm{O}$ & $\begin{array}{l}\text { Barbituric acid (s) } \\
\text { A minobarbituric acid (s) }\end{array}$ & $\begin{array}{l}128.05 \\
143.07\end{array}$ & & $\begin{array}{l}358.7 \\
379.1\end{array}$ & $\begin{array}{l}1,501.2 \\
1,586.5\end{array}$ & & $\begin{array}{l}66 ; \text { cf. } 130 . \\
1300\end{array}$ \\
\hline $\mathrm{C}_{4} \mathrm{H}_{6} \mathrm{~N}_{4} \mathrm{O}_{3}$ & Allantoin (s) ${ }^{38}$ & 158. 09 & & 413.5 & $1,730.5$ & & 130. \\
\hline $\mathrm{C}_{5} \mathrm{H}_{4} \mathrm{~N}_{4} \mathrm{O}$ & Hypoxanthine (s) (6-Oxy- & 136. 06 & & 821.0 & $3,435.9$ & & $\begin{array}{l}0.9 \\
19 .\end{array}$ \\
\hline $\mathrm{C}_{5} \mathrm{H}_{4} \mathrm{~N}_{4} \mathrm{O}$ & 8-Oxypurine (s) & 136.06 & & 591.8 & $2,476.7$ & & 19. \\
\hline $\mathrm{C}_{5} \overline{\mathrm{H}}_{4} \mathrm{~N}_{4} \mathrm{O}$ & $\begin{array}{c}\text { Xanthine } \\
\text { purine). }\end{array}$ & 152.07 & & 514.6 & $2,153.6$ & & 24. \\
\hline $\mathrm{C}_{5} \mathrm{H}_{4} \mathrm{~N}_{4} \mathrm{O}_{3}$ & Uric acid $(\mathrm{s})^{38}$ & 168. 07 & & 460.2 & $1,925.9$ & & 194; cf. 130 , \\
\hline $\begin{array}{l}\mathrm{C}_{5} \mathrm{H}_{5} \mathrm{~N}_{5} \mathrm{O} \\
\mathrm{C}_{5} \mathrm{H}_{6} \mathrm{~N}_{2} \mathrm{O}\end{array}$ & Guanine (s) - & 151. 01 & & 586.4 & $2,454.1$ & & 194. \\
\hline $\mathrm{C}_{5} \mathrm{H}_{6} \mathrm{I}$ & $\begin{array}{l}\text { 4-Methyluracil (s) } \\
\text { 5-Methyluracil (s) (Natural }\end{array}$ & $\begin{array}{l}\text { 126. } 07 \\
126.07\end{array}$ & & $\begin{array}{l}566.1 \\
564.8\end{array}$ & $\begin{array}{l}2,371.6 \\
2,366.1\end{array}$ & & $\begin{array}{l}241 ; \text { cf. } 66 . \\
66 .\end{array}$ \\
\hline $\mathrm{C}_{5} \mathrm{H}_{6} \mathrm{~N}_{2} \mathrm{O}_{3--}$ & Dimethylparabanic acid (s).. & 142.07 & & 538.3 & $2,252.8$ & & 130. \\
\hline $\mathrm{C}_{5} \mathrm{H}_{6} \mathrm{~N}_{4} \mathrm{C}$ & Pseudouric acid (s) & 186.10 & & 454.2 & $1,900.8$ & & 130. \\
\hline $\mathrm{C}_{5} \mathrm{H}_{8} \mathrm{~N}_{2} \mathrm{O}_{2}$ & 4-Methylhydrouracil (s) & 128.08 & & 618.2 & $2,589.8$ & & 66. \\
\hline $\mathrm{C}_{6}$ & 7-Methylpurine (s) & 134.08 & & 821.0 & $3,435.9$ & & 19. \\
\hline & 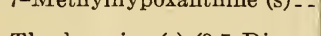 & -100.00 & & 109.8 & $0,100.2$ & & 19. \\
\hline $\mathrm{C}_{7} \mathrm{H}_{8} \mathrm{~N}_{4} \mathrm{O}_{2} \ldots$ & $\begin{array}{l}\text { Theobromine (s) (3,7-Dime- } \\
\text { thyl-2,6-dihydroxypurine). }\end{array}$ & 180. 11 & & 845.3 & $3,537.6$ & & 130. \\
\hline $\mathrm{C}_{8} \mathrm{H}_{6} \mathrm{~N}_{4} \mathrm{O}_{8} .2 \mathrm{H}_{2} \mathrm{O}$ & Alloxanthine (s) & 322.12 & & 583.9 & $2,443.6$ & & 130. \\
\hline $\mathrm{C}_{8} \mathrm{H}_{8} \mathrm{~N}_{6} \mathrm{O}_{6} \ldots$ & Murexide (s) - & 284.12 & & & & & 130. \\
\hline $\mathrm{C}_{3} \mathrm{H}_{10} \mathrm{~N}_{4} \mathrm{O}_{2} \ldots$ & $\begin{array}{l}\text { Caffeine (s) }(1,3,7-\text { Trimethyl- } \\
\text { xanthine). }\end{array}$ & 194.12 & & $1,014.2$ & $4,244.4$ & & 194. \\
\hline $\mathrm{C}_{8} \mathrm{H}_{12} \mathrm{~N}_{2} \mathrm{O}_{3}$ & Veronal (s) & 184. 12 & & 983.1 & $4,113.3$ & & 66. \\
\hline $\begin{array}{l}\mathrm{C}_{10} \mathrm{H}_{8} \mathrm{~N}_{2} \mathrm{C} \\
\mathrm{C}_{12} \mathrm{H}_{14} \mathrm{~N}_{4}\end{array}$ & 4-Phenyluracil. (s) - & 188. 08 & & $1,131.0$ & $4,737.8$ & & 66. \\
\hline $\mathrm{C}_{12} \mathrm{H}_{14} \mathrm{~N}_{4} \mathrm{O}$ & Tetramethylalloxanthine (s) & $\begin{array}{l}310.14 \\
342.15\end{array}$ & & $1,239.3$ & $5,186.5$ & & $\begin{array}{l}130 . \\
130 .\end{array}$ \\
\hline & & & & & & & \\
\hline
\end{tabular}

38 The author (130) gives the heat of combustion of methylallantoin, $\mathrm{C}_{5} \mathrm{H}_{8} \mathrm{~N}_{4} \mathrm{O}_{3}$. Note, however, that the analysis for nitrogen shows a wide divergence from the calculated value. The values of (130) are open to serious objections in that he used camphor to aid in the combustion of the substance. No great reliance should be attached to any of his values.

39 The author (130) gives also the heat of combustion of ammonium urate, $\mathrm{C}_{5} \mathrm{H}_{7} \mathrm{~N}_{5} \mathrm{O}_{3}$.

\section{RING NITROGEN COMPOUNDS}

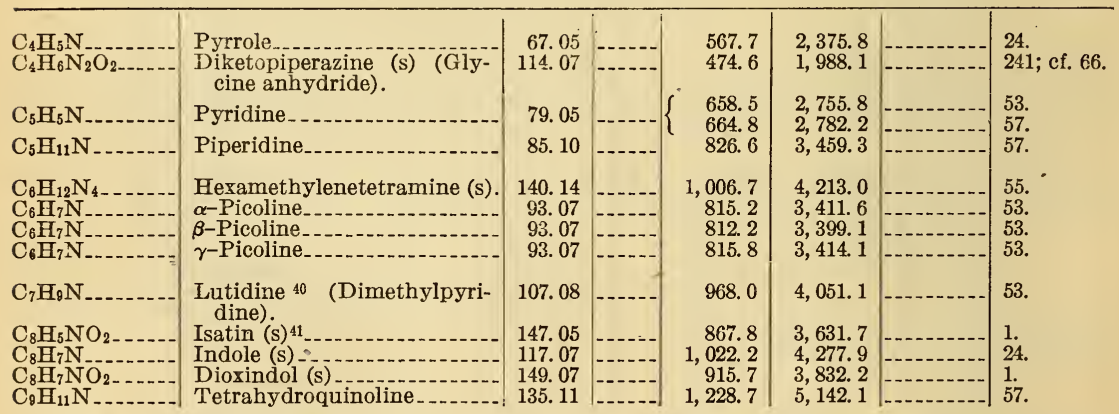

40 The exact isomer not designated in article.

41 The author (1) gives also the heat of combustion of isatide, $\mathrm{C}_{16} \mathrm{H}_{12} \mathrm{~N}_{2} \mathrm{O}_{4}$. obtained by reducing isatin. with zinc dust in acid solution, as $1,777.8 \mathrm{~kg}$-cal 15 per mole. 


\section{TABLES OF DATA-Continued}

\section{N COMPOUNDS-Continued}

68. RING NITROGEN COMPOUNDS-Continued

\begin{tabular}{|c|c|c|c|c|c|c|c|}
\hline Formula & Name & $\begin{array}{c}\text { Molec- } \\
\text { ular } \\
\text { weight }\end{array}$ & $\begin{array}{c}\text { Num- } \\
\text { ber } \\
\text { of } \\
\text { elec- } \\
\text { trons } \\
(N)\end{array}$ & $\begin{array}{l}\mathrm{Kg} \text {-cal } 15 \\
\text { (experi- } \\
\text { mental) }\end{array}$ & $\begin{array}{l}\text { Kilo- } \\
\text { joules } \\
\text { (K. J.) }\end{array}$ & $\begin{array}{l}\mathrm{Kg}-\mathrm{cal}_{15} \\
\text { (calcu- } \\
\text { lated to } \\
\text { the } \\
\text { liquid } \\
\text { state) }\end{array}$ & Literature \\
\hline $\mathrm{C}_{9} \mathrm{H}_{9} \mathrm{~N}$ & Skatole (3-Methylindole) & 131.08 & & $1,170.5$ & $4,898.5$ & & 24. \\
\hline $\mathrm{C}_{9} \mathrm{H}_{9} \mathrm{~N}$ & $\alpha-$ Methylindole & 131.08 & & $1,168.7$ & $4,891.0$ & & 24. \\
\hline $\mathrm{C}_{9} \mathrm{H}_{7} \mathrm{~N}_{-}$ & Quinoline. & 129.06 & & $1,123.5$ & $4,701.9$ & & 57. \\
\hline $\mathrm{C}_{10} \mathrm{H}_{9} \mathrm{~N}$ & Phen ylpyrrole (s) & 143.08 & & $1,283.5$ & $5,371.5$ & - & 183. \\
\hline $\mathrm{C}_{10} \mathrm{H}_{2} \mathrm{~N} \ldots$ & $\begin{array}{l}\text { Quinaldine ( } \alpha \text {-Methylquin- } \\
\text { oline). }\end{array}$ & 143.08 & & $1,287.7$ & $5,389.0$ & & 57. \\
\hline $\mathrm{C}_{10} \mathrm{H}_{9} \mathrm{NO}_{4--}$ & Opianic acid oxime anhy- & 207.08 & & $1,151.4$ & $4,818.6$ & & $183 ;$ cf. 161. \\
\hline $\mathrm{C}_{10} \mathrm{H}_{13} \mathrm{~N}$. & $\begin{array}{l}\text { dride (S). } \\
\text { Tetrahydroquinaldine. }\end{array}$ & 147. 11 & & $1,382.1$ & $5,784.1$ & & 57. \\
\hline $\mathrm{C}_{12} \mathrm{H}_{9} \mathrm{~N}$ & Carbazole (s) & 167.08 & & $1,475.0$ & $6,172.9$ & & 24 . \\
\hline $\mathrm{C}_{16} \mathrm{H}_{10} \mathrm{~N}_{2} \mathrm{O}_{2}$ & Indigo (s) & 262.10 & & $1,815.0$ & $7,595.8$ & $\ldots$ & 1. \\
\hline $\mathrm{C}_{20} \mathrm{H}_{27} \mathrm{NO}_{11 \ldots} \ldots$ & Amygdalin (s) & 457.2 & & $2,348.4$ & $9,828.0$ & & 19. \\
\hline
\end{tabular}

69. RING NITROGEN COMPOUNDS, IMIDES

$Q=26.05 \times N-3.5 \mathrm{hh}$

\begin{tabular}{|c|c|c|c|c|c|c|c|}
\hline $\begin{array}{l}\mathrm{C}_{4} \mathrm{H}_{5} \mathrm{NO}_{2} \ldots \ldots \\
\mathrm{C}_{5} \mathrm{H}_{7} \mathrm{NO}_{4} \ldots \ldots \\
\mathrm{C}_{6} \mathrm{H}_{9} \mathrm{NO}_{4} \ldots \ldots \\
\mathrm{C}_{6} \mathrm{H}_{9} \mathrm{NO}_{4} \ldots \ldots\end{array}$ & $\begin{array}{l}\text { Succinimide (s) } \\
d l-\mathrm{N} \text {-Methyltartrimide }(\mathrm{s}) \\
d-\mathrm{N} \text {-Ethyltartrimide }(\mathrm{s}) \\
d l-\mathrm{N} \text {-Ethyltartrimide }(\mathrm{s})\end{array}$ & $\begin{array}{r}99.05 \\
145.06 \\
159.08 \\
159.08\end{array}$ & $\begin{array}{l}17 \\
19 \\
25 \\
25\end{array}$ & $\begin{array}{l}437.9 \\
516.5 \\
671.1 \\
671.2\end{array}$ & $\begin{array}{l}1,832.6 \\
2,161.6 \\
2,808.6 \\
2,809.0\end{array}$ & $\begin{array}{l}442.8 \\
514.4 \\
670.7 \\
670.7\end{array}$ & $\begin{array}{l}184 ; \text { cf. } 27 \text {. } \\
231 \text {. } \\
231 \text {. } \\
231 \text {. }\end{array}$ \\
\hline $\begin{array}{l}\mathrm{C}_{6} \mathrm{H}_{9} \mathrm{NO}_{4} \ldots \ldots \\
\mathrm{C}_{8} \mathrm{H}_{5} \mathrm{NO}_{2} \ldots \ldots \\
\mathrm{C}_{10} \mathrm{H}_{9} \mathrm{NO}_{4} \ldots \ldots \\
\mathrm{C}_{10} \mathrm{H}_{9} \mathrm{NO}_{4} \ldots \ldots\end{array}$ & $\begin{array}{l}\text { N-Ethylmesotartrimide (s) } \\
\text { Phthalimide }(\mathrm{s}) \\
\text { Hemipinimide }(\mathrm{s}) \\
d-\mathrm{N} \text {-Phenyltartrimide. }\end{array}$ & $\begin{array}{l}159.08 \\
147.05 \\
207.08 \\
207.08\end{array}$ & $\begin{array}{r}25 \\
33 \\
-71\end{array}$ & $\begin{array}{r}672.7 \\
849.5 \\
1,099.0 \\
1,085.7\end{array}$ & $\begin{array}{l}2,811.9 \\
3,555.2 \\
4,599.3 \\
4,543.7\end{array}$ & $\begin{array}{r}670.7 \\
852.6 \\
1,07 \overline{7} .6\end{array}$ & $\begin{array}{l}231 . \\
184 . \\
108 ; \text { cf. } 161 \\
231 .\end{array}$ \\
\hline $\begin{array}{l}\mathrm{C}_{10} \mathrm{H}_{9} \mathrm{NO}_{4} \\
\mathrm{C}_{11} \mathrm{H}_{11} \mathrm{NO}_{4} \\
\mathrm{C}_{11} \mathrm{H}_{11} \mathrm{NO}_{4} \\
\mathrm{C}_{11} \mathrm{H}_{11} \mathrm{NO}_{4} \ldots \ldots\end{array}$ & $\begin{array}{l}d l-\mathrm{N}-\text { Phenyltartrimide } \\
d-\mathrm{N} \text {-Benzyltartrimide }(\mathrm{s})-. \\
d l-\mathrm{N} \text {-Benzyltartrimide }(\mathrm{s}) \\
\text { N-Benzylmesotartrimide }(\mathrm{s})\end{array}$ & $\begin{array}{l}207.08 \\
221.10 \\
221.10 \\
221.10\end{array}$ & $\begin{array}{l}41 \\
47 \\
47 \\
47\end{array}$ & $\begin{array}{l}1,085.9 \\
1,237.8 \\
1,237.7 \\
1,240.7\end{array}$ & $\begin{array}{l}4,544.5 \\
5,180.2 \\
5,179.8 \\
5,192.3\end{array}$ & $\begin{array}{l}1,077.6 \\
1,240.3 \\
1,240.3 \\
1,240.3\end{array}$ & $\begin{array}{l}231 . \\
231 . \\
231 . \\
231 .\end{array}$ \\
\hline
\end{tabular}

70. ALKALOIDS

(Pyridine, Piperidine, Quinoline, and Isoquinoline)

\begin{tabular}{|c|c|c|c|c|c|c|c|}
\hline $\begin{array}{l}\mathrm{C}_{8} \mathrm{H}_{17} \mathrm{~N} \\
\mathrm{C}_{10} \mathrm{H}_{14} \mathrm{~N}_{2} \\
\mathrm{C}_{17} \mathrm{H}_{19} \mathrm{NO}_{3} . \mathrm{H}_{2} \mathrm{O}_{-} \\
\mathrm{C}_{18} \mathrm{H}_{21} \mathrm{NO}_{3} . \mathrm{H}_{2} \mathrm{O}_{-} \\
\mathrm{C}_{19} \mathrm{H}_{21} \mathrm{NO}_{3}\end{array}$ & $\begin{array}{l}\text { Coniine } \\
\text { Nicotine } \\
\text { Morphine (s) } \\
\text { Codeine (s) } \\
\text { Thebaine (s) }\end{array}$ & $\begin{array}{l}127.14 \\
162.13 \\
303.18 \\
317.20 \\
311.18\end{array}$ & & $\begin{array}{l}1,275.5 \\
1,427.7 \\
2,146.3 \\
2,327.6 \\
2,441.3\end{array}$ & $\begin{array}{r}5,334.1 \\
5,974.9 \\
8,975.8 \\
9,734.0 \\
10,209.5\end{array}$ & $\begin{array}{l}-- \\
-- \\
--\end{array}$ & $\begin{array}{l}19 . \\
24 . \\
106 . \\
106 . \\
106 .\end{array}$ \\
\hline $\begin{array}{l}\mathrm{C}_{20} \mathrm{H}_{21} \mathrm{NO}_{4} \ldots \\
\mathrm{C}_{21} \mathrm{H}_{22} \mathrm{~N}_{2} \mathrm{O}_{2} \ldots \\
\mathrm{C}_{22} \mathrm{H}_{23} \mathrm{NO}_{7} \ldots \\
\mathrm{C}_{23} \mathrm{H}_{26} \mathrm{~N}_{2} \mathrm{O}_{4} \\
\mathrm{C}_{23} \mathrm{H}_{27} \mathrm{NO}_{8.2 \mathrm{H}_{2} \mathrm{O}}\end{array}$ & $\begin{array}{l}\text { Papaverine (s) } \\
\text { Strychnine (s) } \\
\text { Narcotine (s) } \\
\text { Brucine (s) } \\
\text { Narceine (s) }\end{array}$ & $\begin{array}{l}339.18 \\
334.20 \\
413.20 \\
394.23 \\
481.27\end{array}$ & $\begin{array}{ll}-\cdots \\
-\cdots \\
-\cdots\end{array}$ & $\begin{array}{l}2,478.1 \\
2,685.7 \\
2,644.5 \\
2,933.0 \\
2,802.9\end{array}$ & $\begin{array}{l}10,363.4 \\
11,239.7 \\
11,059.3 \\
12,274.6 \\
11,721.7\end{array}$ & 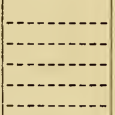 & $\begin{array}{l}106 . \\
28 . \\
106 . \\
28 \\
106\end{array}$ \\
\hline
\end{tabular}

71. ALIPHATIC NITRILES

$Q=26.05 \times N+16.5$

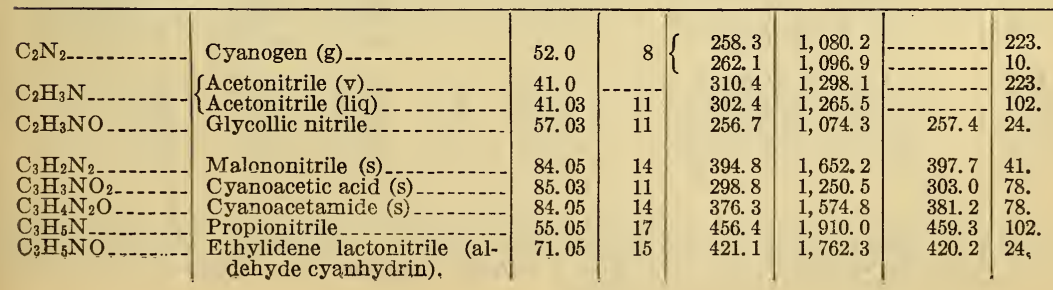




\section{TABLES OF DATA-Continued}

\section{IN COMPOUNDS-Continued}

\section{ALIPHATIC NITRILES-Continued}

$Q=26.05 \times N+16.5$

\begin{tabular}{|c|c|c|c|c|c|c|c|}
\hline Formula & Name & $\begin{array}{c}\text { Molec- } \\
\text { ular } \\
\text { weight }\end{array}$ & $\begin{array}{l}\text { Num- } \\
\text { ber } \\
\text { of } \\
\text { elec- } \\
\text { trons }\end{array}$ & $\begin{array}{l}\text { Kg-cal } \\
\text { (experi- } \\
\text { mental) }\end{array}$ & $\begin{array}{l}\text { Kilo- } \\
\text { jouiles } \\
\text { (K. J.) }\end{array}$ & $\begin{array}{l}\text { Kg-cal } \\
\text { (calcu- } \\
\text { lated to } \\
\text { the } \\
\text { liquid } \\
\text { state) }\end{array}$ & Literature \\
\hline $\mathrm{C}_{4} \mathrm{~N}_{2--}$ & $\begin{array}{l}\text { Carbon subnitrido (s) (acet- } \\
\text { ylenedicarboxylic acid nूi- } \\
\text { trile). }\end{array}$ & 76.0 & 16 & ${ }^{42} 514.8$ & $2,152.9$ & 482.9 & 139. \\
\hline $\mathrm{C}_{4} \mathrm{H}_{4} \mathrm{~N}_{2}$ & Succinic acid nitrile.......... & 80.04 & 20 & 545.7 & $2,283.7$ & 554.0 & 41. \\
\hline $\mathrm{C}_{4} \mathrm{H}$ & Allyl cyanide....... & 67.05 & 21 & 574.9 & $2,406.0$ & 576. 6 & 51. \\
\hline $\mathrm{C}_{4} \mathrm{H}_{5} \mathrm{~N}$ & Trimethylene nitrile & 67.05 & 21 & 581.5 & $2,433.6$ & - 576.6 & 51. \\
\hline $\mathrm{C}_{4} \mathrm{H}_{5} \mathrm{~N}_{-}$ & Crotononitrile.............. & 67.05 & 21 & 571.9 & $2,393.4$ & 576.6 & 51. \\
\hline $\mathrm{C}_{4} \mathrm{H}_{5} \mathrm{~N}$ & Isocrotononitrile. & 67.05 & 21 & 573.8 & $2,401.4$ & 576.6 & 51. \\
\hline & Methyl cyanoacetate & 99.05 & 17 & 471.6 & $1,973.7$ & 475.8 & 78. \\
\hline $\mathrm{C}_{4} \mathrm{H}$ & Diglycolamidic nitrile (di- & 95.07 & & 590.6 & $2,471.7$ & & 198. \\
\hline $\begin{array}{l}\mathrm{C}_{4} \mathrm{H}_{7} \mathrm{~N} \\
\mathrm{C}_{5} \mathrm{H}_{6} \mathrm{~N}_{2-}\end{array}$ & $\begin{array}{l}\text { (cyanomethyl)amine) } \\
\text { n-Butyronitrile } \\
\text { Glutaric nitrile }(\mathrm{s})\end{array}$ & $\begin{array}{l}69.07 \\
94.07\end{array}$ & $\begin{array}{l}23 \\
26\end{array}$ & $\begin{array}{l}613.3 \\
699.6\end{array}$ & $\begin{array}{l}2,566.7 \\
2,927.8\end{array}$ & $\begin{array}{l}615.6 \\
710.3\end{array}$ & 102. \\
\hline & & & & & & & \\
\hline $\mathrm{C}_{5} \mathrm{H}_{9} \mathrm{~N}$ & $\begin{array}{l}\text { Ethyl cyanoacetate....... } \\
\text { Isovaleronitrile........ }\end{array}$ & $\begin{array}{r}113.07 \\
83.08\end{array}$ & $\begin{array}{l}23 \\
29\end{array}$ & $\begin{array}{l}630.0 \\
772.1\end{array}$ & $\begin{array}{l}2,636.6 \\
3,231.2\end{array}$ & $\begin{array}{l}635.1 \\
771.9\end{array}$ & $\begin{array}{l}78 . \\
102 .\end{array}$ \\
\hline $\mathrm{C}_{6} \mathrm{H}_{6} \mathrm{~N}_{4-}$ & Triglycolamidic nitrile (tri- & 134.09 & & 846.0 & $3,540.5$ & & 198. \\
\hline $\mathrm{C}_{6} \mathrm{H}_{7} \mathrm{NO}_{3}$ & Methyl acetylcyanoacetato & 141.07 & 25 & 685.0 & $2,866.7$ & 693.7 & 78. \\
\hline $\mathrm{C}_{6} \mathrm{I}$ & $\begin{array}{l}\text { (s). } \\
\text { Propyl cyanoacetate.... }\end{array}$ & 127.08 & 29 & 789.0 & $3,301.9$ & 791.4 & \\
\hline & Ethyl cyanoacetylacetate $(\mathrm{s})$ & 155.08 & 31 & 836. 6 & $3,501.2$ & 850.0 & 78. \\
\hline $\mathrm{C}_{8} \mathrm{~F}$ & Amyl propiolic nitrile....... & 121.09 & 43 & $1,164.2$ & $4,868.7$ & $1,169.7$ & 138. \\
\hline $\mathrm{C}_{11} \mathrm{H}_{15}$ & Cyanocamphor $(\mathrm{s})$ & 177.05 & 57 & $1,405.4$ & $6,258.3$ & $1,507.8$ & 42. \\
\hline
\end{tabular}

42 The above value represents the result of a single determination and should be considered merely as an approximate value.

43 Considerable difficulty was experienced in the combustion of the compound due to its explosive character.

${ }_{44} \mathrm{~N} \equiv\left(\mathrm{CH}_{2} \mathrm{C} \equiv \mathrm{N}\right)_{3}$.

\section{AROMATIC NITRILES}

$Q=26.05 \times N+16.5 \mathrm{nn}-6.5 \mathrm{pp}$

\begin{tabular}{|c|c|c|c|c|c|c|c|}
\hline $\mathrm{C}_{7} \mathrm{H}_{5} \mathrm{~N}_{-}$ & Benzonitrile. & 103.05 & 33 & 865.5 & $3,622.1$ & 869.6 & 41. \\
\hline $\mathrm{C}_{8} \mathrm{H}_{7} \mathrm{~N}$ & Benzyl cyanide.- & 117.07 & 39 & $1,023.5$ & $4,283.4$ & $1,029.4$ & 41. \\
\hline $\mathrm{C}_{8} \mathrm{H}_{7} \mathrm{~N}$ & 0 -Tolunitrile.... & 117.07 & 39 & $1,030.3$ & $4,311.8$ & $1,022.4$ & 41. \\
\hline $\mathrm{C}_{8} \mathrm{H}_{5} \mathrm{NO}_{2} . . .$. & Benzoyl cyanide (s) & 131.05 & 35 & 940.2 & $3,934.7$ & 45937.7 & 78. \\
\hline $\mathrm{C}_{9} \mathrm{H}_{7} \mathrm{NO}$. & Cyanoacetophenone $(\mathrm{s})$....... & 145.07 & & $1,085.6$ & $4,543.2$ & & 78. \\
\hline $\mathrm{C}_{0} \mathrm{H}_{5} \mathrm{~N}_{--}$ & Phenylpropiolic nitrile & 127.04 & 41 & $1,117.8$ & $\overrightarrow{4}, 674.6$ & $1,111.2$ & 138. \\
\hline $\mathrm{C}_{11} \mathrm{H}_{7} \mathrm{~N}$ & $\alpha-N a p h t h o n i t r i l e(s)$ & 153.07 & 51 & $1,326.2$ & $5,550.2$ & $1,325.5$ & 102. \\
\hline $\mathrm{C}_{11} \mathrm{H}_{7} \mathrm{~N}_{-} \ldots$ & $\beta$-Naphthonitrile (s) & 153.07 & 51 & $1,321.0$ & $5,528.4$ & $1,325.5$ & 102. \\
\hline
\end{tabular}

${ }^{45}$ See formula for ketonic acids.

73. CARBYLAMINES

(Aliphatic)

$\mathrm{Q}=26.05 \times N+33.1$ -

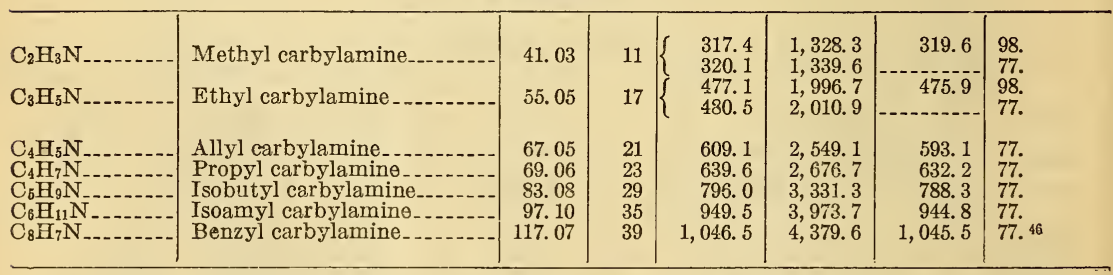

${ }_{46}$ Th3 same article contains also the heats of combustion of double compounds of silver cyanide with methyl, ethyl, propyl, isobutyl, isoamyl carbylamines, $\mathrm{C}_{3} \mathrm{H}_{3} \mathrm{~N}_{2} \mathrm{Ag}$ to $\mathrm{C}_{7} \mathrm{H}_{11} \mathrm{~N}_{2} \mathrm{Ag}$. The heats of combus tion of these compounds are, within the limits of experimental error, rather uniformly about 503 kilojoules larger than the values for the carbylamines. 


\section{TABLES OF DATA-Continued}

\section{N COMPOUNDS-Continued}

74. ISOCYANATES

(Aliphatic)

$Q=26.05 \times N+33.1$

\begin{tabular}{|c|c|c|c|c|c|c|c|}
\hline Formula & Name & $\begin{array}{l}\text { Molec- } \\
\text { ular } \\
\text { weight }\end{array}$ & $\begin{array}{c}\text { Num- } \\
\text { ber } \\
\text { of } \\
\text { elec- } \\
\text { trons }\end{array}$ & $\begin{array}{l}\mathrm{Kg}_{\text {-cal }}{ }_{15} \\
\text { (experi- } \\
\text { mental) }\end{array}$ & $\begin{array}{c}\text { Kilo- } \\
\text { joules } \\
\text { (K. J.) }\end{array}$ & $\begin{array}{l}\mathrm{Kg} \text {-cal } 15 \\
\text { (calcu- } \\
\text { lated to } \\
\text { the } \\
\text { liquid } \\
\text { state) }\end{array}$ & Literature \\
\hline $\begin{array}{l}\mathrm{C}_{2} \mathrm{H}_{3} \mathrm{NO} \\
\mathrm{C}_{3} \mathrm{H}_{5} \mathrm{NO}_{\ldots} \ldots \ldots\end{array}$ & $\begin{array}{l}\text { Methyl isocyanate } \\
\text { Ethyl isocyanate }\end{array}$ & $\begin{array}{l}57.03 \\
71.05\end{array}$ & $\begin{array}{r}9 \\
15\end{array}$ & $\begin{array}{l}269.4 \\
424.5\end{array}$ & $\begin{array}{l}1,127.4 \\
1,776.5\end{array}$ & $\begin{array}{l}267.6 \\
423.9\end{array}$ & $\begin{array}{l}96 . \\
96 .\end{array}$ \\
\hline
\end{tabular}

75. HYDROXYLAMINE DERIVATIVES

\begin{tabular}{|c|c|c|c|c|c|c|c|}
\hline $\begin{array}{l}\mathrm{C}_{6} \mathrm{H}_{7} \mathrm{NO}_{2} \\
\mathrm{C}_{6} \mathrm{H}_{6} \mathrm{~N}_{2} \mathrm{O}_{3} \ldots\end{array}$ & $\begin{array}{l}\text { Phenylhydroxylamine }{ }^{47} \\
\text { m-Nitrophenylhydroxylam- } \\
\text { ine }\end{array}$ & $\begin{array}{l}109.06 \\
154.06\end{array}$ & $\left|\begin{array}{c}-\cdots \\
-\cdots-1\end{array}\right|$ & $\begin{array}{l}803.7 \\
765.6\end{array}$ & $\begin{array}{l}3,366.7 \\
3,204.0\end{array}$ & - & $\begin{array}{l}163 . \\
164 .\end{array}$ \\
\hline
\end{tabular}

47 The author (163) does not indicate which isomer is burned.

76. ALIPHATIC NITRO COMPOUNDS

$$
Q=25.05 \times N+13
$$

\begin{tabular}{|c|c|c|c|c|c|c|c|}
\hline $\begin{array}{l}\mathrm{CH}_{3} \mathrm{NO}_{2} \ldots \ldots \\
\mathrm{C}_{2} \mathrm{H}_{5} \mathrm{NO}_{2} \ldots \ldots \\
\mathrm{C}_{3} \mathrm{H}_{7} \mathrm{NO}_{2} \ldots \ldots \\
\mathrm{C}_{10} \mathrm{H}_{15} \mathrm{NO}_{3} \ldots\end{array}$ & $\begin{array}{l}\text { Nitromethane } \\
\text { Nitroethane } \\
\text { Nitropropane } \\
\alpha-N i t r o c a m p h o r(s)\end{array}$ & $\begin{array}{r}61.03 \\
75.05 \\
89.07 \\
197.13\end{array}$ & $\begin{array}{r}6 \\
12 \\
18 \\
--\sim\end{array}$ & $\begin{array}{r}169.4 \\
322.2 \\
477.9 \\
1,371.1\end{array}$ & $\begin{array}{r}708.4 \\
1,348.4 \\
1,998.6 \\
5,738.1\end{array}$ & $\begin{array}{r}169.3 \\
325.6 \\
481.9 \\
\end{array}$ & $\begin{array}{l}212 ; \text { cf. } 36 . \\
36 . \\
212 \text {. } \\
42.48\end{array}$ \\
\hline
\end{tabular}

$48 \mathrm{This}$ article contains also the heat of combustion of other forms of nitrocamphor.

\section{AROMATIC NITRO COMPOUNDS 49}

$Q=26.05 \times N+13 s s$

\begin{tabular}{|c|c|c|c|c|c|c|c|}
\hline $\mathrm{F}$ & Titrobenzene & & $28+2$ & 739 & $3,091.3$ & 742.4 & \\
\hline $\mathrm{CH}$ & Nolodenzede...... & & 28 & 732. & $3,062.9$ & $-\ldots-1$ & \\
\hline $\mathrm{C}_{6} \mathrm{H}_{4} \mathrm{~N}_{2} \mathrm{O}$ & o-Dinitrobenzene (s) & 168.05 & 26 & 703. 2 & $2,942.9$ & 703.3 & 33 ; cf. 72 . \\
\hline $4+\sqrt{2} \mathrm{O}_{4}$ & $p$-Dinitrobenzene (s) & 168.05 & 26 & 695.1 & 2, 908. 9 & 703.3 & 33. \\
\hline $\mathrm{C}_{6} \mathrm{H}_{4} \mathrm{~N}_{2} \mathrm{O}$ & $m$-Dinitrobenzene (s) ${ }^{50}$ & 168.05 & 26 & $\begin{array}{l}696.8 \\
694.7\end{array}$ & $\begin{array}{l}2,916.1 \\
2,905.2\end{array}$ & 703.3 & $\begin{array}{l}33 . \\
211 \text {; cf. } 72 .\end{array}$ \\
\hline $\begin{array}{l}\mathrm{C}_{6} \mathrm{H}_{3} \mathrm{~N}_{3} \mathrm{O}_{6} \\
\mathrm{C}_{6} \mathrm{H}_{3} \mathrm{~N}_{3} \mathrm{O}_{6} \ldots \ldots \\
\mathrm{C}_{19} \mathrm{H}_{13} \mathrm{~N}_{3} \mathrm{O}_{6} \ldots . . \\
\mathrm{C}_{19} \mathrm{H}_{13} \mathrm{~N}_{3} \mathrm{O}_{7 \ldots}\end{array}$ & $\begin{array}{l}\text { 1,3,5-Trinitrobenzene (s) } \\
\text { 1,2,4-Trinitrobenzene (s) } \\
\text { Trinitrotriphenylmethane(s) } \\
\text { Trinitrotriphenyl carbinol(s) }\end{array}$ & $\begin{array}{l}213.05 \\
213.05 \\
379.13 \\
395.13\end{array}$ & $\begin{array}{l}24 \\
24 \\
86 \\
84\end{array}$ & $\begin{array}{r}663.7 \\
673.7 \\
2,271.5 \\
2.216 .7\end{array}$ & $\begin{array}{l}2,777.6 \\
2,817.4 \\
9,499.4 \\
9,270.2\end{array}$ & $\begin{array}{r}664.2 \\
664.7 \\
2,279.3 \\
2,230.7\end{array}$ & $\begin{array}{l}33 ; \text { cf. } 72 . \\
72 . \\
179 . \\
179 .\end{array}$ \\
\hline
\end{tabular}

40 The heats of combustion of 1,3,5-trinitrobenzene, 2,4,6-trinitro-1-methylnitroaminobenzene, $\mathrm{C}_{7} \mathrm{H}_{5} \mathrm{~N}_{5} \mathrm{O}_{8}$, 2,4,6-trinitrotoluene, $\mathrm{C}_{7} \mathrm{H}_{5} \mathrm{~N}_{3} \mathrm{O}_{6}, 2,3,4,6$-tetranitro-l-methylnitroaminobenzene, $\mathrm{C}_{7} \mathrm{H}_{4} \mathrm{~N}_{6} \mathrm{O}_{10}$, ammonium picrate, $\mathrm{C}_{6} \mathrm{H}_{6} \mathrm{~N}_{4} \mathrm{O}_{7}$, ammonium salt of hexanitrodiphenylamine, $\mathrm{C}_{12} \mathrm{H}_{8} \mathrm{~N}_{8} \mathrm{O}_{12}$, and trinitroethane, $\mathrm{C}_{2} \mathrm{H}_{3} \mathrm{~N}_{3} \mathrm{O}_{7}$, used as explosives, are given by Rubtzov and Sever' yanov, J. Russ. Phys. Chem. Soc., כ̃ 0, p. 140; 1918. However, the results are valueless as far as accuracy is concerned, and, since the data in regard to the methods employed are entirely missing, the values have been omitted from this report.

${ }_{50}$ The author (148) claims that by mixing $m$-dinitrobenzene and naphthalene no heat is evolved. Certainly the lower heat of combustion 0.18 per cent obtained for the mixture is meaningless for individual determinations do not check better than 0.5 to 1 per cent. 


\section{TABLES OF DATA-Continued}

\section{N COMPOUNDS-Continued}

78. SUBSTITUTED AROMATIC NITRO COMPOUNDS

$Q=26.05 \times N+13 \mathrm{ss}-3.5 \mathrm{a}$

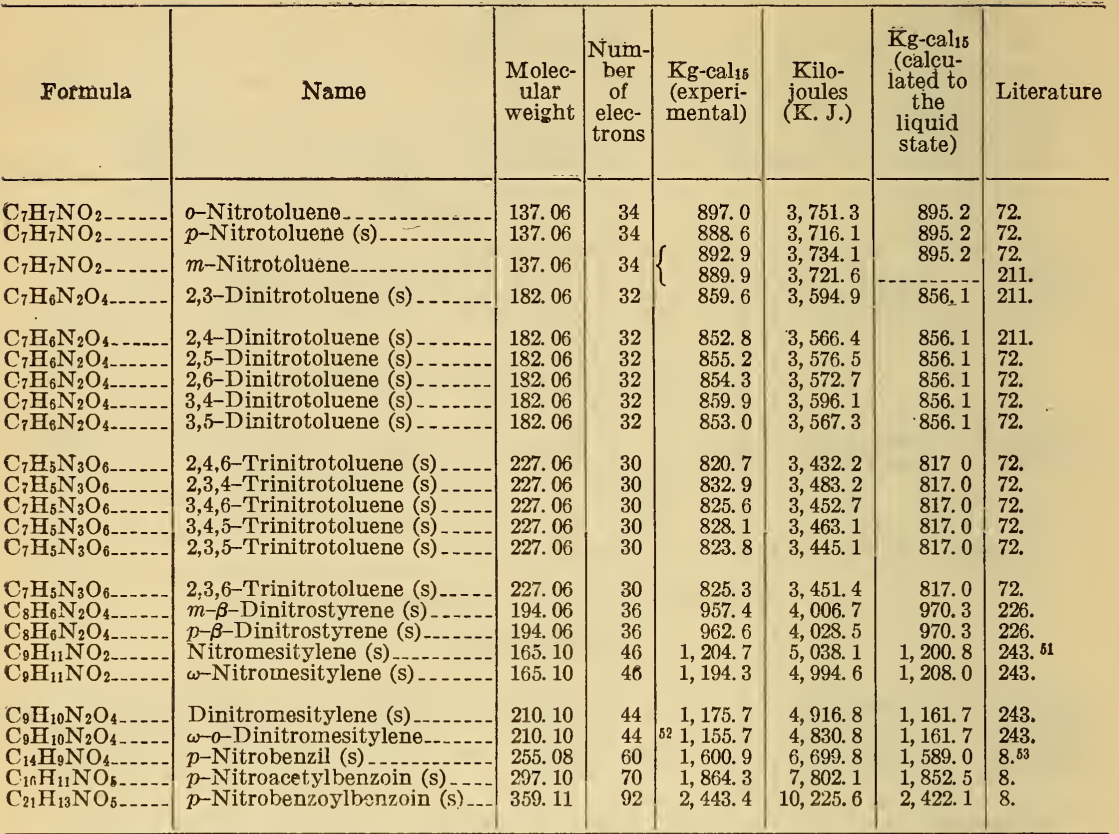

31 All the values by (243) have been corrected according to the method suggested by Swietoslawski, J. Amer. Chem. Soc., 42, p. 1091; 1920.

52 The author (243) believes that the difference in the heats of combustion of these two isomers, 1.79 per cent, is due to the fact that the compounds were not quite pure.

${ }^{53}$ Details as to procedure employed in obtaining the data are completely lacking in the paper.

79. AROMATIC NITROPHENOLS, PHENETOLES, NITRANILINES, NITROACETANILIDES

$Q=26.05 \times N+13 \mathrm{ss}+3.5 \mathrm{~m}+6.5 \mathrm{j}+13 \mathrm{kk}+19.5 \mathrm{p}$

\begin{tabular}{|c|c|c|c|c|c|c|c|}
\hline $\mathrm{C}_{6} \mathrm{H}_{5} \mathrm{NO}_{3} \ldots \ldots$ & o-Nitrophenol (s) & 139.05 & 26 & 689.1 & $\begin{array}{l}2,881.8 \\
2,878.9\end{array}$ & $\begin{array}{r}693.8 \\
\end{array}$ & 72. \\
\hline $\mathrm{C}_{6} \mathrm{H}_{5} \mathrm{NO}_{3}$ & $m$-Nitrophenol (s) & 139.05 & 26 & 684.4 & $2,864.2$ & 693.8 & 210. \\
\hline $\mathrm{C}_{6} \mathrm{H}_{5} \mathrm{NO}_{3}$ & $p$-Nitrophenol (s) & 139.05 & 26 & $\begin{array}{l}688.8 \\
688.2\end{array}$ & $\begin{array}{l}2,882.6 \\
2,880.1\end{array}$ & - & $\begin{array}{l}131 . \\
210 .\end{array}$ \\
\hline $\mathrm{C}_{6} \mathrm{H}_{4} \mathrm{~N}_{2} \mathrm{O}_{5}$ & 2,4-Dinitrophenol (s) & 184.05 & 24 & 648.0 & $2,709.9$ & 654.7 & 72. \\
\hline $\mathrm{C}_{6} \mathrm{H}_{3} \mathrm{~N}_{3} \mathrm{O}_{7}$ & $\begin{array}{l}2,4,6 \text {-Trinitrophenol (s) (Pic- } \\
\text { ric acid). }\end{array}$ & 229.05 & 22 & 621.1 & $2,597.4$ & 615.6 & 72. \\
\hline $\mathrm{C}_{8} \mathrm{H}_{9} \mathrm{NO}$ & o-Nitrophenetole.............. & 167.08 & 38 & 1, 021. 2 & $4,270.7$ & $1,022.4$ & 211. \\
\hline $\mathrm{C}_{8} \mathrm{H}_{92}$ & $m$-Nitrophenetole. & 167.08 & 38 & $1,009.2$ & $4,223.5$ & $1,022.4$ & 210. \\
\hline $\mathrm{C}_{8} \mathrm{H}_{9} \mathrm{NO}_{3}$ & p-Nitrophenotole_. & 167.08 & 38 & $1,006.0$ & $4,210.1$ & $1,022.4$ & 210. \\
\hline $\mathrm{C}_{6} \mathrm{H}_{6} \mathrm{~N}_{2} \mathrm{O}_{2}$ & o-Nitraniline (s) & 138. 06 & 29 & 765.8 & $3,204.8$ & 771.4 & 210 . \\
\hline $\mathrm{C}_{6} \mathrm{H}_{6} \mathrm{~N}_{2} \mathrm{O}_{2--}$ & $m-N i t r a n i l i n e(s)$ & 138. 06 & 29 & 765. 2 & $3,200.1$ & 771.4 & 211. \\
\hline $\mathrm{C}_{6} \mathrm{H}_{6} \mathrm{~N}$ & $p$-Nitraniline (s) & 138.06 & 29 & 761.0 & $3,187.8$ & 771.4 & 170. \\
\hline $\mathrm{C}_{7} \mathrm{H}_{8} \mathrm{~N}_{2} \mathrm{O}_{2}$ & $p$-Nitromethylaniline (s) & 152. 08 & 35 & 924.3 & 3.865 .4 & 934.3 & 72. \\
\hline $\mathrm{C}_{7} \mathrm{H}_{7} \mathrm{~N}$ & 2,4-Dinitromethylaniline (s) & 197.08 & 33 & 884.5 & $3,698.9$ & 895.2 & 72. \\
\hline $\mathrm{C}_{7} \mathrm{H}_{6} \mathrm{~N}_{4} \mathrm{O}_{6}$ & 2,4,6-Trinitromethylaniline & 242.08 & 31 & 857.9 & $3,587.7$ & 856. 0 & 72. \\
\hline $\mathrm{C}_{7} \mathrm{H}_{5} \mathrm{~N}_{5} \mathrm{O}_{8}$ & Tetryl (s) & 287.08 & & 842.3 & $3,522.5$ & & 72. \\
\hline $\mathrm{C}_{8} \mathrm{H}_{7} \mathrm{~N}_{5} \mathrm{O}_{8}$ & Methyltetryl (s) (2,4,6-Tri- & 301.10 & & $1,009.3$ & $4,220.9$ & & 72. \\
\hline $\mathrm{C}_{8} \mathrm{H}_{8} \mathrm{~N}_{2} \mathrm{O}_{3}$ & 0 -Nitroacetanilide (s) & 180.08 & 37 & 973.9 & $4,075.8$ & 973.3 & 210. \\
\hline $\mathrm{C}_{8} \mathrm{H}_{8} \mathrm{~N}_{2} \mathrm{O}_{3}$ & $m$-Nitroacetanilide (s) & 180. 08 & 37 & 969.5 & $4,054.5$ & 973.3 & 211 ; cf. 131. \\
\hline $\mathrm{C}_{8} \mathrm{H}_{8} \mathrm{~N}_{2} \mathrm{O}_{3} \ldots$ & $p$-Nitroacetanilide (s) & 180.08 & 37 & 968.2 & $4,051.9$ & 973.3 & 210 . \\
\hline
\end{tabular}




\section{TABLES OF DATA-Continued}

\section{N COMPOUNDS-Continued}

\section{AROMATIC NITROALDEHYDES}

$Q=26.05 \times N+13 \mathrm{ss}+13 \mathrm{r}-3.5 \mathrm{a}$

\begin{tabular}{c|r|r|r|r|r|r|r}
\hline Formula & Name & $\begin{array}{c}\text { Molec- } \\
\text { ular } \\
\text { weight }\end{array}$ & $\begin{array}{c}\text { Num- } \\
\text { ber } \\
\text { of } \\
\text { elec- } \\
\text { trons }\end{array}$ & $\begin{array}{c}\text { Kg-cal } \\
\text { (experi- } \\
\text { mental) }\end{array}$ & $\begin{array}{c}\text { Kilo- } \\
\text { joules } \\
\text { (K. J.) }\end{array}$ & $\begin{array}{c}\text { Kg-cal15 } \\
\text { (calcu- } \\
\text { lated to } \\
\text { the } \\
\text { liquid } \\
\text { state) }\end{array}$ & Literature \\
\hline $\mathrm{C}_{7} \mathrm{H}_{5} \mathrm{NO}_{3-1 .--}$ & $m$-Nitrobenzaldehyde (s) & 151.05 & 30 & 800.4 & $3,349.7$ & 804.0 & 131. \\
\hline
\end{tabular}

\section{AROMATIC NITRO ACIDS}

$Q=26.05 \times N+13 \mathrm{ss}-3.5 \mathrm{a}+13 \mathrm{c}$

\begin{tabular}{|c|c|c|c|c|c|c|c|}
\hline $\mathrm{C}_{7} \mathrm{H}_{5} \mathrm{NO}_{4} \ldots \ldots$ & o-Nitrobenzoic acid (s) $\ldots \ldots$ & 167.05 & 28 & $\left\{\begin{array}{l}729.8 \\
735.0\end{array}\right.$ & $\begin{array}{l}3,054.2 \\
3,078.9\end{array}$ & 738.9 & $\begin{array}{l}131 . \\
151 .\end{array}$ \\
\hline $\mathrm{C}_{7} \mathrm{H}_{5} \mathrm{NO}_{4}-$ & $m$-Nitrobenzoic acid (s) $\ldots \ldots$ & 167.05 & 28 & $\left\{\begin{array}{l}726.4 \\
729.1\end{array}\right.$ & $\begin{array}{l}3,039.9 \\
3,049.1\end{array}$ & 738.9 & 58. \\
\hline $\mathrm{C}_{7} \mathrm{H}_{5} \mathrm{NO}_{4} \ldots$ & $p$-Nitrobenzoic acid (s) & 167.05 & 28 & $\left\{\begin{array}{l}728.3 \\
728.3\end{array}\right.$ & $\begin{array}{l}3,047.9 \\
3,045.8\end{array}$ & 738.9 & 58. \\
\hline $\begin{array}{l}\mathrm{C}_{9} \mathrm{H}_{7} \mathrm{NO}_{4} \ldots \ldots \\
\mathrm{C}_{9} \mathrm{H}_{7} \mathrm{NO}_{4} \ldots \ldots \\
\mathrm{C}_{9} \mathrm{H}_{7} \mathrm{NO}_{4} \ldots \ldots\end{array}$ & $\begin{array}{l}\text { o-Nitrocinnamic acid (s) } \\
m-\mathrm{Nitrocinnamic} \text { acid (s) } \\
p-\mathrm{Nitrocinnamic} \text { acid (s) } \ldots\end{array}$ & $\begin{array}{l}193.06 \\
193.06 \\
193.06\end{array}$ & $\begin{array}{l}38 \\
38 \\
38\end{array}$ & $\begin{array}{l}999.0 \\
995.6 \\
996.5\end{array}$ & $\begin{array}{l}4,180.8 \\
4,166.6 \\
4,170.4\end{array}$ & $\begin{array}{l}1,009.4 \\
1,009.4 \\
1,009.4\end{array}$ & $\begin{array}{l}226 . \\
226 . \\
226 .\end{array}$ \\
\hline
\end{tabular}

\section{PHENYLHYDRAZONES AND OSAZONES}

The two articles by $\mathrm{Ph}$. Landrieu, Compt. rend., 141, p. 358; 1905; 142, p. 540; 1906, contain the heats of combustion of the phenylhydrazones of the following aldehydes and ketones: Acetaldehyde, acetone, diacetyl, furfuraldehyde, benzaldehyde, salicylaldehyde, acetophenone, anisaldehyde, benzophenone, benzoin, benzil; the phenylhydrazones of the following sugars: Arabinose, glucose, galactose, levulose, mannose, maltose, lactose; the osazones of glyoxal, diacetyl, benzil; and of the following sugars: Arabinose, xylose, glucose, levulose, mannose, galactose, maltose, lactose. The data are not included in the tables, for the information given in the above articles in regard to the work is extremely meager, and a careful analysis of the values indicates that they do not form a homogeneous series.

83. ALDOXIMES (ALIPHATIC)

\begin{tabular}{|c|c|c|c|c|c|c|c|}
\hline $\begin{array}{l}\mathrm{C}_{2} \mathrm{H}_{5} \mathrm{NO} \\
\mathrm{C}_{3} \mathrm{H}_{7} \mathrm{NO}\end{array}$ & $\begin{array}{l}\text { Aldoxime (s) } \\
\text { Acetoxime (s) }\end{array}$ & $\begin{array}{l}59.05 \\
73.07\end{array}$ & - & $\begin{array}{l}340.6 \\
490.5\end{array}$ & $\begin{array}{l}1,424.4 \\
2,051.3\end{array}$ & $-\cdots$ & $\begin{array}{l}93.54 \\
93 .\end{array}$ \\
\hline
\end{tabular}

.54 It is of interest to compare these values of (93) with those of $(215)$ for methyl ethyl ketoxime, $\mathrm{C}_{4} \mathrm{H}_{0} \mathrm{NO}$. The former's values appear to be about 0.8 per cent too high.

\section{KETOXIMES (ALIPHATIC)}

\begin{tabular}{|c|c|c|c|c|c|c|c|}
\hline $\mathrm{C}_{4} \mathrm{H}_{9} \mathrm{NO}$ & Methyl ethyl ketoxime...... & $\begin{array}{r}87.08 \\
155.15\end{array}$ & & $\left\{\begin{array}{r}646.5 \\
651.0 \\
363.5\end{array}\right.$ & $\begin{array}{l}2,703.7 \\
2,724.4 \\
5,702.2\end{array}$ & & 215. \\
\hline $\mathrm{C}_{10} \mathrm{H}_{17} \mathrm{NO}$ & $\begin{array}{l}\text { ime (s). } \\
\text { Camphoroxime (s) }\end{array}$ & $\begin{array}{l}155.15 \\
167.15\end{array}$ & & $\begin{array}{l}1,363.5 \\
1,480.8\end{array}$ & $\begin{array}{l}5,702.2 \\
6,197.2\end{array}$ & & $\begin{array}{l}215 . \\
93 .\end{array}$ \\
\hline
\end{tabular}




\section{TABLES OF DATA-Continued}

\section{N COMPOUNDS-Continued}

\section{AROMATIC ALDOXIMES AND KETOXIMES}

\begin{tabular}{|c|c|c|c|c|c|c|c|}
\hline Formula & Name & $\begin{array}{c}\text { Molec- } \\
\text { ular } \\
\text { weight }\end{array}$ & \begin{tabular}{|} 
Num- \\
ber \\
of \\
elec- \\
trons
\end{tabular} & $\begin{array}{l}\mathrm{Kg} \text {-cal } 15 \\
\text { (experi- } \\
\text { mental) }\end{array}$ & $\begin{array}{l}\text { Kilo- } \\
\text { joules } \\
(\mathrm{K} . \mathrm{J} .)\end{array}$ & $\begin{array}{l}\text { Kg-cal } 15 \\
\text { (calcu- } \\
\text { lated to } \\
\text { the } \\
\text { liquid } \\
\text { state) }\end{array}$ & Literature \\
\hline $\mathrm{C}_{6} \mathrm{H}_{5} \mathrm{NO}_{2} \ldots \ldots$ & $\begin{array}{l}\text { Benzoquinone oxime ( } p-\mathrm{Ni}- \\
\text { trosophenol). }\end{array}$ & 123.05 & & 713.5 & $2,985.9$ & & 225 ; cf. 222. \\
\hline $\mathrm{C}_{7} \mathrm{H}_{7} \mathrm{NO}_{-}$ & Benzaldoxime (s) & 121. 07 & & 907.2 & $3,793.9$ & & 93. \\
\hline $\mathrm{C}_{8} \mathrm{H}_{9} \mathrm{NO}_{-}$ & Methyl phenyl ketoxime (s) & 135.08 & & $1,054.4$ & $4,409.5$ & $\cdots$ & \\
\hline $\mathrm{C}_{10} \mathrm{H}_{7} \mathrm{NO}_{2}$ & $\begin{array}{l}\alpha-\mathrm{Naph} \text { thoquinone oxime } \\
\text { (s) ( } \alpha \text {-Nitroso- } \alpha-n \text { aph- } \\
\text { thol). }\end{array}$ & 173.07 & & $1,163.5$ & $4,868.3$ & -- & 225 ; cf. 222. \\
\hline $\mathrm{C}_{10} \mathrm{H}_{7} \mathrm{NO}_{2} \ldots \ldots$ & $\begin{array}{l}\beta \text {-Naphthoquinone oxime } \\
\text { (s) }(\beta \text {-Nitroso- } \alpha \text {-naph- } \\
\text { thol). }\end{array}$ & 173.07 & & $1,167.4$ & $4,885.6$ & & 225; cf. 222. \\
\hline $\mathrm{C}_{10} \mathrm{H}_{7} \mathrm{NO}_{2} \ldots$ & $\begin{array}{l}\beta \text {-Naphthoquinone oxime } \\
\text { (s) ( } \alpha \text {-Nitroso- } \beta \text {-naph- } \\
\text { thol). }\end{array}$ & 173.07 & & $1,166.7$ & $4,882.6$ & & 225 ; cf. 222. \\
\hline $\begin{array}{l}\mathrm{C}_{10} \mathrm{H}_{13} \mathrm{NO}_{2-} \\
\mathrm{C}_{13} \mathrm{H}_{11} \mathrm{NO}_{--}\end{array}$ & $\begin{array}{l}\text { Oxime of opianic anhydride }{ }^{55} \\
\text { Thymoquinone oxime }(\mathrm{s}) \\
\text { Diphenyl ketoxime (s) }\end{array}$ & 179.11 & & $1,152.3$ & $\begin{array}{l}4,822.4 \\
5,573.6\end{array}$ & & 108. 225 ; cf. 222. \\
\hline & Diphenyl ketoxime (s) & 197.10 & & $1,626.8$ & $6,803.3$ & & $225 ;$ cf. 93. \\
\hline
\end{tabular}

55 Stohmann, communicated by Liebermann (Ber., 25 , p. 89; 1892.) Formula and molecular weight not indicated. He does not specify whether the above value refers to constant volume or constant pressure.

\section{NITROSAMINES 56}

\begin{tabular}{|c|c|c|c|c|c|c|c|}
\hline $\begin{array}{l}\mathrm{C}_{2} \mathrm{H}_{6} \mathrm{~N}_{2} \mathrm{O} \\
\mathrm{C}_{3} \mathrm{H}_{10} \mathrm{~N}_{2} \mathrm{O} \\
\mathrm{C}_{12} \mathrm{H}_{10} \mathrm{~N}_{2} \mathrm{O} \ldots\end{array}$ & $\begin{array}{l}\text { Dimethylnitrosamine } \\
\text { Ethylphenylnitrosamine (s)- } \\
\text { Diphenylnitrosamine (s) }\end{array}$ & $\begin{array}{r}74.07 \\
150.10 \\
198.10\end{array}$ & & $\begin{array}{r}394.5 \\
1,118.4 \\
1,532.6\end{array}$ & $\begin{array}{l}1,650.9 \\
4,680.5 \\
6,413.9\end{array}$ & & $\begin{array}{l}212 . \\
212 . \\
132\end{array}$ \\
\hline
\end{tabular}

${ }^{56}$ See also article by Delépine (C. R., 123, p. 650; 1896) for nitroso and nitro compounds of hexanuethylene tetramine.

\section{NITRAMINES}

\begin{tabular}{|c|c|c|c|c|c|c|c|}
\hline $\mathrm{C}_{2} \mathrm{H}_{6} \mathrm{~N}_{2} \mathrm{O}_{2} \ldots \ldots$ & Ethylnitramine ${ }^{67} \ldots$ & 80.00 & $-\ldots$ & 372.5 & $1,557.8$ & & 211. \\
\hline
\end{tabular}

${ }^{87} \mathrm{CH}_{3} . \mathrm{CH}_{2} \mathrm{NH} . \mathrm{NO}_{2}$.

\section{NITROSO COMPOUNDS}

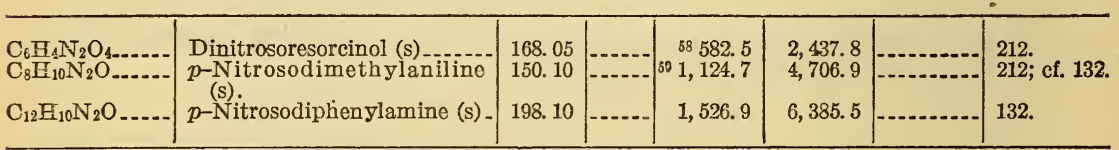

68 The author (212) gives as the mean value $3,473 \mathrm{cal} / \mathrm{g}$. In his calculation of the molecular heat of combustion he employs 186 as the molecular weight instead of 168.05 . The article contains, also, the heats of reaction of a large number of compounds with nitrous acid. For a detailed treatment of this subject consult Ber., 43, p. 1470; 1910; 44, p. 2429; 1911.

50 The author (212) gives also the heat of combustion of nitrosodimethylaniline hydrochloride as $4,687.2$ kilo-joules.

\section{AZO COMPOUNDS}

\begin{tabular}{|c|c|c|c|c|c|c|c|}
\hline $\mathrm{C}_{12} \mathrm{H}_{10} \mathrm{~N}_{2} \ldots \ldots$ & Azobenzene (s) & 182.10 & & $\left\{\begin{array}{l}1,555.2 \\
1,552.6 \\
1,545.9\end{array}\right.$ & $\begin{array}{l}6,508.5 \\
6,497.6 \\
6,469.6\end{array}$ & & 147. \\
\hline $\mathrm{C}_{12} \mathrm{H}_{10} \mathrm{~N}_{2} \mathrm{O}$. & $p$-Hydroxyazobenzene (s) _... & 198. 10 & & $1,502.0$ & $6,285.9$ & & 101. \\
\hline $\mathrm{C}_{12} \mathrm{H}_{11} \mathrm{~N}_{3} \ldots \ldots$ & p-Aminoazobenzene (s) & 197.12 & & $1,574.0$ & $6,587.2$ & $\ldots$ & 101. \\
\hline $\mathrm{C}_{12} \mathrm{H}_{12} \mathrm{~N}_{4} \ldots \ldots$ & $\begin{array}{l}\text { 2,4-Diaminoazobenzene (s) } \\
\text { (Chrysoidine). }\end{array}$ & 212.14 & & $1,597.4$ & $6,685.1$ & -- & 101. \\
\hline $\begin{array}{l}\mathrm{C}_{14} \mathrm{H}_{14} \mathrm{~N}_{2} \mathrm{O}_{2} \ldots \\
\mathrm{C}_{16} \mathrm{H}_{18} \mathrm{~N}_{2} \mathrm{O}_{2} \ldots \ldots\end{array}$ & $p$-Azoanisole & 242.13 & & $1,796.4$ & $7,517.9$ & & 101. \\
\hline $\mathrm{C}_{16} \mathrm{H}_{18 \mathrm{~N}_{2}} \mathrm{O}_{2} \ldots$ & $p$-Azophenetole (S) & 270.16 & & $2,100.0$ & $8,788.5$ & -- & 101. \\
\hline
\end{tabular}

60 Undoubtedly the best value is that of (217), 


\section{TABLES OF DATA-Continued}

\section{N COMPOUNDS-Continued}

90. SUBSTITUTED HYDRAZINES

\begin{tabular}{|c|c|c|c|c|c|c|c|}
\hline Formula & Name & $\begin{array}{l}\text { Molec- } \\
\text { ular } \\
\text { weight }\end{array}$ & $\begin{array}{l}\text { Num- } \\
\text { ber } \\
\text { of } \\
\text { elec- } \\
\text { trons }\end{array}$ & $\begin{array}{l}\mathrm{Kg} \text {-cal } 15 \\
\text { (experi- } \\
\text { mental) }\end{array}$ & $\begin{array}{c}\text { Kilo- } \\
\text { joules } \\
\text { (K. J.) }\end{array}$ & $\begin{array}{l}\mathrm{Kg}-\mathrm{cal}_{15} \\
\text { (calcu- } \\
\text { lated to } \\
\text { the } \\
\text { liquid } \\
\text { state) }\end{array}$ & Literature \\
\hline $\mathrm{C}_{6} \mathrm{H}_{8} \mathrm{~N}_{2 \ldots} \ldots$ & Phenylhydrazine (s) & 108.08 & & $\left\{\begin{array}{l}875.4 \\
805.4\end{array}\right.$ & $3,663.6$ & 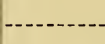 & 100. \\
\hline $\mathrm{C}_{7} \mathrm{H}_{10} \mathrm{~N}_{2}$ & $\begin{array}{l}\text { Methylphenylhydrazine (s) } \\
\text { (asym.). }\end{array}$ & 122.10 & & $1,038.1$ & $\begin{array}{l}3,310.0 \\
4,344.5\end{array}$ & & 100. \\
\hline $\mathrm{C}_{12} \mathrm{H}_{12} \mathrm{~N}_{2 \ldots}$ & Hydrazobenzene (s) & 184. 12 & & $\left\{\begin{array}{l}1,597.3 \\
1,598.8\end{array}\right.$ & $\begin{array}{l}6,684.7 \\
6,690.9\end{array}$ & - & $\begin{array}{l}100 . \\
147 .\end{array}$ \\
\hline
\end{tabular}

91. DIAZONIUM COMPOUNDS

\begin{tabular}{l|l|l|l|l|l|l|}
\hline $\mathrm{C}_{6} \mathrm{H}_{5} \mathrm{~N}_{3} \mathrm{O}_{3} \ldots \ldots-\ldots$ & $\begin{array}{l}\text { Benzenediazonium nitrate } \\
\text { (s). }\end{array}$ & 167.07 & 782.6 & $3,275.2$ & $\ldots . \ldots$ \\
\hline
\end{tabular}

92. AZOXY COMPOUNDS

\begin{tabular}{|c|c|c|c|c|c|c|c|}
\hline $\mathrm{C}_{12} \mathrm{H}_{10} \mathrm{~N}_{2} \mathrm{O}_{\ldots} \ldots$ & Azoxybenzene (s) -..- & 198.10 & & $\left\{\begin{array}{l}1,534.5 \\
1,530.1\end{array}\right.$ & $\begin{array}{l}6,421.9 \\
6,403.5\end{array}$ & $-\ldots$ & $\begin{array}{l}101 . \\
147\end{array}$ \\
\hline $\begin{array}{l}\mathrm{C}_{14} \mathrm{H}_{16} \mathrm{~N}_{4} \mathrm{O}_{2} \\
\mathrm{C}_{16} \mathrm{H}_{18} \mathrm{~N}_{2} \mathrm{O}_{3} \ldots \\
\mathrm{C}_{16} \mathrm{H}_{18} \mathrm{~N}_{2} \mathrm{O}_{3} \ldots\end{array}$ & $\begin{array}{l}m \text {-Azoxytoluidine (s) } \\
p \text {-Azoxyphenetole (s) } \\
\text { o-Azoxyphenetole (s) }\end{array}$ & $\begin{array}{l}256.17 \\
286.13 \\
286.13\end{array}$ & 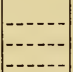 & $\begin{array}{l}1,903.4 \\
2,098.4 \\
2,088.0\end{array}$ & $\begin{array}{l}7,965.7 \\
8,781.8 \\
8,730.3\end{array}$ & 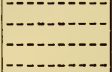 & $\begin{array}{l}101 . \\
101 . \\
101 .\end{array}$ \\
\hline
\end{tabular}

93. ESTERS OF NITRIC AND NITROUS ACID

\begin{tabular}{|c|c|c|c|c|c|c|c|}
\hline $\begin{array}{l}\mathrm{C}_{2} \mathrm{H}_{5} \mathrm{NO}_{2} \ldots \\
\mathrm{C}_{2} \mathrm{H}_{5} \mathrm{NO}_{3} \ldots \ldots \\
\mathrm{C}_{3} \mathrm{H}_{5} \mathrm{~N}_{3} \mathrm{O}_{9} \ldots \ldots \\
\mathrm{C}_{4} \mathrm{H}_{9} \mathrm{NO}_{2} \ldots \ldots\end{array}$ & $\begin{array}{l}\text { Ethyl nitrite }(\nabla) \\
\text { Ethyl nitrate }(\nabla) \\
\text { Trinitroglycerol } \\
\text { Isobutyl nitrite }(\nabla)\end{array}$ & $\begin{array}{l}75.0 \\
91.0 \\
227.06 \\
103.0\end{array}$ & 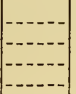 & $\begin{array}{l}332.6 \\
322.4 \\
432.4 \\
644.6\end{array}$ & $\begin{array}{l}1,390.9 \\
1,348.3 \\
1,809.6 \\
2,695.7\end{array}$ & $\mid \overline{u-n-2-2}$ & $\begin{array}{l}223 . \\
223 . \\
81 . \\
223 .\end{array}$ \\
\hline
\end{tabular}

\section{HALOGEN AND SULPEUR COMPOUNDS}

\section{FLUORINE COMPOUNDS}

(a) FLUORO-HYDROCARBONS (ALIPHATIC)

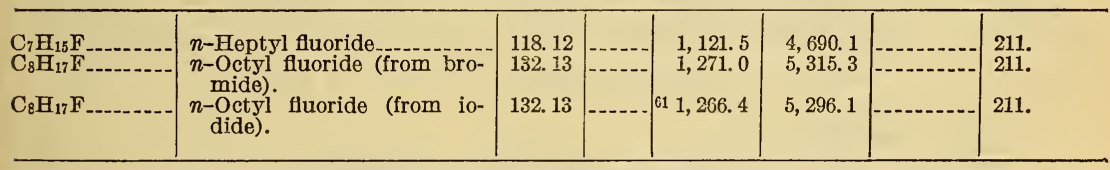

${ }_{61}$ The B. P. of the product prepared from the iodide is $0.6^{\circ}$ higher than the one prepared from the bro mide. See also Ber., 55, p. 3378; 1922.

(B) FLUORO-HYDROCARBONS (AROMATIC)

\begin{tabular}{|c|c|c|c|c|c|c|c|}
\hline $\begin{array}{l}\mathrm{C}_{6} \mathrm{H}_{5} \mathrm{~F} \\
\mathrm{C}_{6} \mathrm{H}_{4} \mathrm{~F}_{2} \\
\mathrm{C}_{7} \mathrm{H}_{7} \mathrm{~F} \\
\mathrm{C}_{7} \mathrm{H}_{7} \mathrm{~F} \\
\mathrm{C}_{7} \mathrm{H}_{6} \mathrm{~F}_{2} \\
\mathrm{C}_{7} \mathrm{H}_{5} \mathrm{~F}_{3} \\
\mathrm{C}_{9} \mathrm{H}_{11} \mathrm{~F}_{\ldots}\end{array}$ & $\begin{array}{l}\text { Fluorobenzene } \\
p \text {-Difluorobenzene } \\
0 \text { - Fluorotoluene } \\
p \text {-Fluorotoluene } \\
\text { w-Difluorotoluene (s) } \\
\text { w-Trifluorotoluene (s) } \\
\text { Fluoropseudocumene (s) }\end{array}$ & $\begin{array}{l}96.05 \\
114.05 \\
110.05 \\
110.05 \\
128.05 \\
146.05 \\
138.1\end{array}$ & 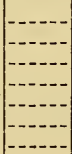 & $\begin{array}{r}747.2 \\
706.1 \\
993.2 \\
903.0 \\
856.9 \\
810.3 \\
1,208.5\end{array}$ & $\begin{array}{l}3,124.8 \\
2,952.9 \\
3,777.2 \\
3,776.3 \\
3,583.6 \\
3,388.7 \\
5,053.9\end{array}$ & 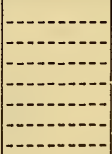 & $\begin{array}{l}211 . \\
211 . \\
211 . \\
211 . \\
211 . \\
211 . \\
211 .\end{array}$ \\
\hline
\end{tabular}

(C) FLUORO-ALCOHOIS (ALIPHATIC)

\begin{tabular}{|c|c|c|c|c|c|c|c|}
\hline $\begin{array}{l}\mathrm{C}_{2} \mathrm{H}_{5} \mathrm{OF} \\
\mathrm{C}_{2} \mathrm{H}_{4} \mathrm{OF}_{2}\end{array}$ & $\begin{array}{l}\text { Fluoroethanol } \\
\text { Difluoroethanol_.. }\end{array}$ & $\begin{array}{l}64.05 \\
82.02\end{array}$ & 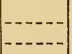 & $\begin{array}{l}290.4 \\
245.8\end{array}$ & $\begin{array}{l}1,214.5 \\
1,027.9\end{array}$ & & 211. \\
\hline
\end{tabular}




\section{TABLES OF DATA-Continued \\ 4. HALOGEN AND SULPHUR COMPOUNDS-Continued}

94. FLUORINE COMPOUNDS-Continued

(D) FLUORO-PHENOLS

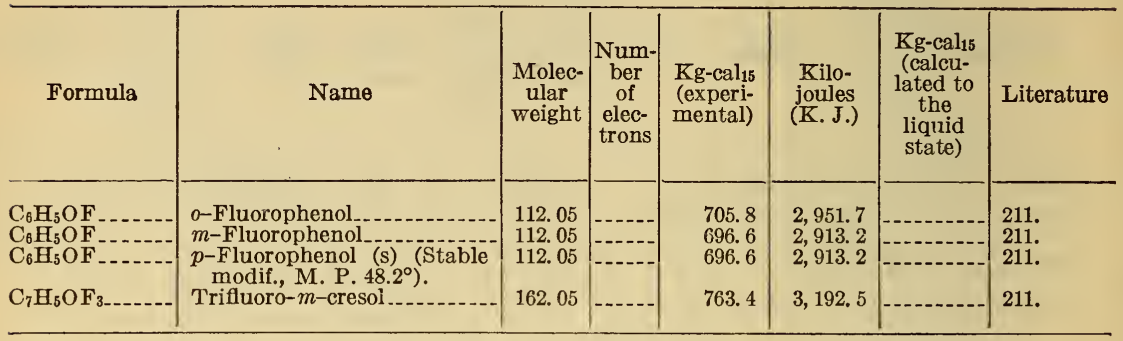

(E) ESTERS OF FLUORO-ALCOHOLS

\begin{tabular}{|c|c|c|c|c|c|c|c|}
\hline $\begin{array}{l}\mathrm{C}_{4} \mathrm{E}_{7} \mathrm{O}_{2} \mathrm{~F} \\
\mathrm{C}_{4} \mathrm{H}_{6} \mathrm{O}_{2} \mathrm{~F}_{2} \ldots \ldots\end{array}$ & $\begin{array}{l}\text { Fluoroethyl acetate } \\
\text { Difluoroethyl acetate...... }\end{array}$ & $\begin{array}{l}106.05 \\
124.05\end{array}$ & & $\begin{array}{l}499.9 \\
455.5\end{array}$ & $\begin{array}{l}2,090.6 \\
1,904.9\end{array}$ & 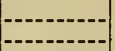 & $\begin{array}{l}211 . \\
211 .\end{array}$ \\
\hline
\end{tabular}

(F) ETHERS OF FLUORO-ALCOHOLS

\begin{tabular}{|c|c|c|c|c|c|c|c|}
\hline $\mathrm{C}_{3} \mathrm{H}_{9} \mathrm{OF} \ldots$ & Ethyl fluoroallyl ether....... & 104.07 & - & 731.0 & $3,057.0$ & & 211. \\
\hline
\end{tabular}

(G) ETHERS OF TLUORO-PHENOLS

\begin{tabular}{|c|c|c|c|c|c|c|c|}
\hline $\begin{array}{l}\mathrm{C}_{8} \mathrm{H}_{9} \mathrm{OF} \\
\mathrm{C}_{8} \mathrm{H}_{9} \mathrm{OF}\end{array}$ & $\begin{array}{l}m \text {-Fluorophenetol } \\
p \text {-Fluorophenetol }\end{array}$ & $\begin{array}{l}140.05 \\
140.05\end{array}$ & 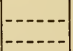 & $\begin{array}{l}1,023.1 \\
1,019.9\end{array}$ & $\begin{array}{l}4,278.6 \\
4,265.2\end{array}$ & 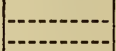 & $\begin{array}{l}211 . \\
211 .\end{array}$ \\
\hline
\end{tabular}

(H) FLUORO-ACIDS ALIPHATIC

\begin{tabular}{|c|c|c|c|c|c|c|c|}
\hline $\begin{array}{l}\mathrm{C}_{2} \mathrm{H}_{3} \mathrm{O}_{2} \mathrm{~F} \\
\mathrm{C}_{2} \mathrm{H}_{2} \mathrm{O}_{2} \mathrm{~F}_{2}\end{array}$ & $\begin{array}{l}\text { Fluoroacetic acid (s) } \\
\text { Difluoroacetic acid }\end{array}$ & $\begin{array}{l}78.02 \\
96.02\end{array}$ & & $\begin{array}{l}170.8 \\
134.6\end{array}$ & $\begin{array}{l}714.3 \\
562.9\end{array}$ & 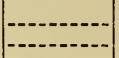 & $\begin{array}{l}211 . \\
211 .\end{array}$ \\
\hline
\end{tabular}

(I) FLUORO-ACIDS (AROMATIC)

\begin{tabular}{|c|c|c|c|c|c|c|c|}
\hline $\mathrm{C}_{7} \mathrm{H}_{5} \mathrm{O}_{2} \mathrm{~F}$ & o-Fluorobenzoic acid (s) & 140.05 & -5 & 740.2 & $3,095.5$ & & 211. \\
\hline $\mathrm{C}_{7} \mathrm{H}_{5} \mathrm{O}_{2} \mathrm{~F}_{-.-}$ & $m$-Fluorobenzoic acid $(\mathrm{s}) \ldots$ & 140.05 & - & 737. 6 & $3,084.6$ & 1--..- & 211. \\
\hline $\mathrm{C}_{7} \mathrm{H}_{5} \mathrm{O}_{2} \mathrm{~F}$ & $p$-Fluorobenzoic acid (s) & 140.05 & $---\infty$ & 739.7 & $3,093.4$ & -- & 211. \\
\hline $\mathrm{C}_{8} \mathrm{H}_{5} \mathrm{O}_{2} \mathrm{~F}_{3}$ & $\begin{array}{l}m \text { - } \omega-\text { Trifluorotoluic acid } 61 \mathrm{a} \\
\text { (s). }\end{array}$ & 190.05 & -- & 807.1 & $3,375.4$ & $-\cdots$ & 211. \\
\hline $\mathrm{C}_{8} \mathrm{H}_{7} \mathrm{O}_{2} \mathrm{~F}$ & $\alpha$-Fluorocinnamic acid (s) $\ldots$ & 166.06 & & $1,013.6$ & $4,238.9$ & & 211. \\
\hline
\end{tabular}

\footnotetext{
61a $m-\mathrm{F}_{3} \mathrm{C}-\mathrm{C}_{6} \mathrm{H}_{4}$. $\mathrm{COOH}$.
}

(J) ESTERS OF FLUORO-ACIDS (ALIPHATIC)

\begin{tabular}{|c|c|c|c|c|c|c|c|}
\hline $\begin{array}{l}\mathrm{C}_{4} \mathrm{H}_{7} \mathrm{O}_{2} \mathrm{~F} \\
\mathrm{C}_{4} \mathrm{H}_{6} \mathrm{O}_{2} \mathrm{~F}_{2}\end{array}$ & Ethyl fluoroacetate & $\begin{array}{l}106.05 \\
124.05\end{array}$ & - & $\begin{array}{l}503.1 \\
436.5\end{array}$ & $\begin{array}{l}2,103.9 \\
1,825.4\end{array}$ & & $\begin{array}{l}211 . \\
211 .\end{array}$ \\
\hline
\end{tabular}

(E) ESTERS OF FLUORO-ACIDS (AROMATIC)

\begin{tabular}{|c|c|c|c|c|c|c|c|}
\hline $\begin{array}{l}\mathrm{C}_{9} \mathrm{H}_{9} \mathrm{O}_{2} \mathrm{~F} \\
\mathrm{C}_{10} \mathrm{H}_{9} \mathrm{O}_{2} \mathrm{~F}\end{array}$ & $\begin{array}{l}\text { Ethyl } p \text {-fluorobenzoate (s)-- } \\
\text { Methyl } \alpha \text {-fluorocinnamate } \\
\text { (s). }\end{array}$ & $\begin{array}{l}168.05 \\
180.05\end{array}$ & -- & $\begin{array}{l}1,060.1 \\
1,187.5\end{array}$ & $\begin{array}{l}4,433.3 \\
4,966.1\end{array}$ & - & $\begin{array}{l}211 . \\
211 .\end{array}$ \\
\hline
\end{tabular}

(L) FLUORO-AMIDES (ALIPHATIC)

\begin{tabular}{|c|c|c|c|c|c|c|c|}
\hline $\begin{array}{l}\mathrm{C}_{2} \mathrm{H}_{4} \mathrm{ONF} \\
\mathrm{C}_{2} \mathrm{H}_{3} \mathrm{ONF} \\
2 \ldots\end{array}$ & $\begin{array}{l}\text { Fluoroacetamide (s) } \\
\text { Difluoroacetamide (s) }\end{array}$ & $\begin{array}{l}77.05 \\
95.05\end{array}$ & - & $\begin{array}{l}250.0 \\
208.3\end{array}$ & $\begin{array}{r}1,045.5 \\
871.1\end{array}$ & 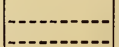 & $\begin{array}{l}211 . \\
211 .\end{array}$ \\
\hline
\end{tabular}




\section{TABLES OF DATA-Continued}

\section{HALOGEN AND SULPHUR COMPOUNDS-Continued}

94. FLUORINE COMPOUNDS-Continued

(M) FLUORO-AMIDES (AROMATIC)

\begin{tabular}{|c|c|c|c|c|c|c|c|}
\hline Formula & Namo & $\begin{array}{c}\text { Molec- } \\
\text { ular } \\
\text { weight }\end{array}$ & $\begin{array}{c}\text { Num- } \\
\text { ber } \\
\text { of } \\
\text { elec- } \\
\text { trons }\end{array}$ & $\begin{array}{l}\text { Kg-cal15 } \\
\text { (experi- } \\
\text { mental) }\end{array}$ & $\begin{array}{l}\text { Kilo- } \\
\text { joules } \\
\text { (K. J.) }\end{array}$ & $\begin{array}{c}\text { Kg-cal } 15 \\
\text { (calcu- } \\
\text { lated to } \\
\text { the } \\
\text { liquid } \\
\text { state) }\end{array}$ & Literature \\
\hline $\begin{array}{l}\mathrm{C}_{8} \mathrm{H}_{7} \mathrm{ONF}_{2} \\
\mathrm{C}_{9} \mathrm{H}_{8} \mathrm{ONF}_{3}\end{array}$ & $\begin{array}{l}\text { Difluoroacetanilide (s) } \\
m \text {-Trifluoroacettoluide (s) } \ldots-\end{array}$ & $\begin{array}{l}171.06 \\
203.75\end{array}$ & $\mid-1-1$ & $\begin{array}{r}944.8 \\
1,043.2\end{array}$ & $\begin{array}{l}3,951.2 \\
4,362.7\end{array}$ & $-\cdots$ & $\begin{array}{l}211 . \\
211 .\end{array}$ \\
\hline
\end{tabular}

(N) FLUORO-AMINES (ALIPHATIC)

\begin{tabular}{|c|c|c|c|c|c|c|c|}
\hline $\begin{array}{l}\mathrm{C}_{2} \mathrm{H}_{5} \mathrm{NF}_{2} \ldots \ldots \\
\mathrm{C}_{4} \mathrm{H}_{7} \mathrm{NFF}_{4} \ldots \ldots\end{array}$ & $\begin{array}{l}\text { Difluoroethylamine. } \\
\text { Tetrafluorodiethylamine.... }\end{array}$ & $\begin{array}{r}81.05 \\
145.06\end{array}$ & & $\begin{array}{l}326.1 \\
566.1\end{array}$ & $\begin{array}{l}1,363.8 \\
2,367.4\end{array}$ & & $\begin{array}{l}211 . \\
211 .\end{array}$ \\
\hline
\end{tabular}

(O) FLUORO-NITRAMINES (ALIPHATIC)

\begin{tabular}{l|l}
$\mathrm{C}_{2} \mathrm{H}_{4} \mathrm{O}_{2} \mathrm{~N}_{2} \mathrm{~F}_{2 \ldots}$ & $\begin{array}{l}\text { Difluoroethylnitramine } \\
\left(\mathrm{MI} . \mathrm{P} .22 .4^{\circ}\right) .\end{array}$
\end{tabular}

(P) FLUORO-NITROBENZENES

\begin{tabular}{|c|c|c|c|c|c|c|c|}
\hline $\begin{array}{l}\mathrm{C}_{6} \mathrm{H}_{4} \mathrm{O}_{2} \mathrm{NF} \\
\mathrm{C}_{6} \mathrm{H}_{4} \mathrm{O}_{2} \mathrm{NF} \\
\mathrm{C}_{6} \mathrm{H}_{4} \mathrm{O}_{2} \mathrm{NF} \\
\mathrm{C}_{6} \mathrm{H}_{3} \mathrm{O}_{2} \mathrm{NF}_{2} \\
\mathrm{C}_{6} \mathrm{H}_{3} \mathrm{O}_{4} \mathrm{~N}_{2} \mathrm{~F}_{\ldots}\end{array}$ & $\begin{array}{l}\text { o-Fluoronitrobenzene } \\
\text { m-Fluoronitrobenzene.-. } \\
\text { p-Fluoronitrobenzene (s) } \\
\text { 1,4-Difluoro-2-nitrobenzene } \\
\text { 2, 4-Dinitro-1-fluorobenzene } \\
\text { (s). }\end{array}$ & $\begin{array}{l}141.05 \\
141.05 \\
141.05 \\
159.04 \\
186.05\end{array}$ & 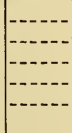 & $\begin{array}{l}707.5 \\
706.4 \\
703.0 \\
673.1 \\
669.3\end{array}$ & $\begin{array}{l}2,958.8 \\
2,954.2 \\
2,939.9 \\
2,814.9 \\
2,799.0\end{array}$ & 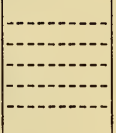 & $\begin{array}{l}211 . \\
211 . \\
211 . \\
211 . \\
211 .\end{array}$ \\
\hline
\end{tabular}

(Q) FLUORO-NITROTOLUENES

\begin{tabular}{|c|c|c|c|c|c|}
\hline $\mathrm{C}_{7} \mathrm{H}_{4} \mathrm{O}_{2} \mathrm{NF}_{3} \ldots$ & $m$-Nitrobenzotrifluoride..... & 191.05 & 771.5 & $3,226.4$ & 211. \\
\hline
\end{tabular}

(R) FLUORO-ANILINES

\begin{tabular}{|c|c|c|c|c|c|c|c|}
\hline $\begin{array}{l}\mathrm{C}_{6} \mathrm{H}_{6} \mathrm{NF} \\
\mathrm{C}_{6} \mathrm{H}_{6} \mathrm{NF} \\
\mathrm{C}_{6} \mathrm{H}_{6} \mathrm{NF}\end{array}$ & $\begin{array}{l}o-\text { Fluoroaniline } \\
m \text {-Fluoroaniline } \\
p \text {-Fluoroaniline. }\end{array}$ & $\begin{array}{l}111.05 \\
111.05 \\
111.05\end{array}$ & $\mid$ & $\begin{array}{l}778.2 \\
771.1 \\
782.2\end{array}$ & $\begin{array}{l}3,254.4 \\
3,249.8 \\
3,271.2\end{array}$ & 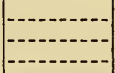 & $\begin{array}{l}211 . \\
211 . \\
211 .\end{array}$ \\
\hline
\end{tabular}

(s) FLUORO-ANILIDES

\begin{tabular}{|c|c|c|c|c|c|c|c|}
\hline $\begin{array}{l}\mathrm{C}_{8} \mathrm{H}_{3} \mathrm{ONF} \\
\mathrm{C}_{8} \mathrm{H}_{8} \mathrm{ONF} \ldots\end{array}$ & $\begin{array}{l}m \text {-Fluoroacetanilide (s) } \\
p \text {-Fluoroacetanilide (s) } \ldots . . .\end{array}$ & $\begin{array}{l}153.07 \\
153.07\end{array}$ & 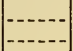 & $\begin{array}{l}978.1 \\
978.9\end{array}$ & $\begin{array}{l}4,090.4 \\
4,093.8\end{array}$ & -- & $\begin{array}{l}211 . \\
211 .\end{array}$ \\
\hline
\end{tabular}

(T) FLUORO-NITROPIENOLS AND NITROPHENETOLES

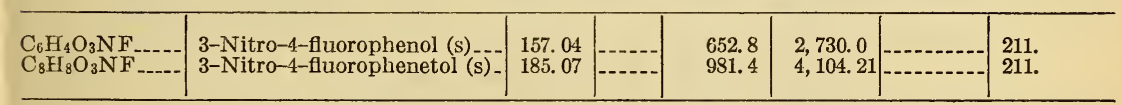

(U) FLUORO-NITRANILINES AND NITRACETANILIDES

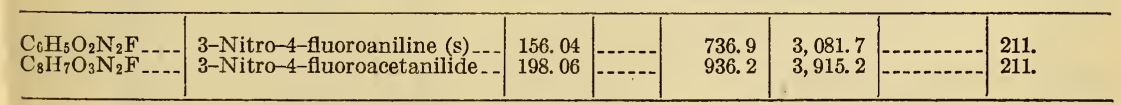




\section{TABLES OF DATA-Continued}

\section{HALOGEN AND SULPHUR COMPOUNDS-Continued}

95. CHLORINE COMPOUNDS

(A) CHLORINATED HYDROCARBONS 62

\begin{tabular}{|c|c|c|c|c|c|c|c|}
\hline Formula & Name & $\begin{array}{l}\text { Molec- } \\
\text { ular } \\
\text { weight }\end{array}$ & $\begin{array}{l}\text { Num- } \\
\text { ber } \\
\text { of } \\
\text { elec- } \\
\text { trons }\end{array}$ & $\begin{array}{l}\text { Kg-cal } \\
\text { (experi- } \\
\text { mental) }\end{array}$ & $\begin{array}{l}\text { Kilo- } \\
\text { joules } \\
\text { (K. J.) }\end{array}$ & $\begin{array}{l}\text { Kg-calis } \\
\text { (calcu- } \\
\text { lated to } \\
\text { the } \\
\text { liquid } \\
\text { state) }\end{array}$ & Literature \\
\hline $\mathrm{CH}_{3} \mathrm{Cl}_{-}$ & Methyl chloride (g) & 50.5 & & $\left\{\begin{array}{l}164.2 \\
173.2\end{array}\right.$ & 686.7 & & 223. \\
\hline $\begin{array}{l}\mathrm{CH}_{2} \mathrm{Cl}_{2-} \\
\mathrm{CHCl} \mathrm{Cl}_{3-}\end{array}$ & $\begin{array}{l}\text { Methylene chloride }(\nabla) \\
\left\{\begin{array}{l}\text { Chloroform ( }) \\
\text { Chloroform (liq.) }\end{array}\right.\end{array}$ & $\begin{array}{r}85.0 \\
119.5\end{array}$ & & $\begin{array}{r}63106.8 \\
70.3 \\
89.2\end{array}$ & $\begin{array}{l}446.9 \\
293.9 \\
373.3\end{array}$ & & $\begin{array}{l}40 . \\
223 . \\
17 .\end{array}$ \\
\hline $\mathrm{CCl}_{4}$ & $\left\{\begin{array}{l}\text { Carbon tetrachloride (v) } \\
\text { Carbon tetrachloride (liq.) }\end{array}\right.$ & 154.0 & & $\begin{array}{l}44.5 \\
37.3\end{array}$ & $\begin{array}{l}\text { 186. } 2 \\
156.1\end{array}$ & & 17. \\
\hline $\mathrm{C}_{2} \mathrm{H}_{5} \mathrm{Cl}$ & Ethyl chloride $(\nabla)$ & 64.5 & & $\begin{array}{l}316.7 \\
326.9\end{array}$ & $1,324.4$ & & 223. \\
\hline $\mathrm{C}_{2} \mathrm{H}_{4} \mathrm{Cl}_{2-}$ & Ethylene chloride (v) . - & 99.0 & & 271.0 & $1,133.3$ & & 223. \\
\hline $\mathrm{C}_{2} \mathrm{H}_{4} \mathrm{Cl}_{2}$ & $\left\{\begin{array}{l}\text { Ethylidene chloride }(\mathrm{v}) \\
\text { Ethylidene chloride (liq.) }\end{array}\right.$ & 99.0 & & 6. 271.1 & $\begin{array}{l}1,133.7 \\
1,117.8\end{array}$ & & $\begin{array}{l}223 . \\
40 .\end{array}$ \\
\hline $\begin{array}{l}\mathrm{C}_{2} \mathrm{Cl}_{6} \\
\mathrm{C}_{2} \mathrm{H}_{3} \mathrm{Cl}-- \\
\mathrm{C}_{2} \mathrm{Cl}_{4}-\end{array}$ & $\begin{array}{l}\text { Hexachloroethane }(\mathrm{s}) \\
\text { Chloroethylene }(\mathrm{v}) \\
\text { Tetrachloroethylene }\end{array}$ & $\begin{array}{r}237.0 \\
62.5 \\
166.0\end{array}$ & & $\begin{array}{l}110.0 \\
281.5 \\
162.5\end{array}$ & $\begin{array}{r}460.4 \\
1,177.2 \\
680.1\end{array}$ & & $\begin{array}{l}17 . \\
223 . \\
17 .\end{array}$ \\
\hline $\begin{array}{l}\mathrm{C}_{3} \mathrm{H}_{7} \mathrm{Cl}_{-} \\
\mathrm{C}_{3} \mathrm{H}_{6} \mathrm{Cl}_{2}\end{array}$ & Propyl chloride ( $\nabla)$ & $\begin{array}{r}78.5 \\
112.9\end{array}$ & & $\begin{array}{l}478.3 \\
427.8\end{array}$ & $\begin{array}{l}2,000.3 \\
1,789.1\end{array}$ & & $\begin{array}{l}223 . \\
223 .\end{array}$ \\
\hline $\begin{array}{l}\mathrm{C}_{3} \mathrm{H}_{5} \mathrm{Cl} \\
\mathrm{C}_{3} \mathrm{H}_{5} \mathrm{Cl}_{-} \\
\mathrm{C}_{3} \mathrm{H}_{4} \mathrm{Cl}_{2--}\end{array}$ & $\begin{array}{l}\text { Chloropropylene }(\nabla) \\
\text { Allyl chloride }(\nabla) \\
\text { Dichlorotrimethylene.-- }\end{array}$ & $\begin{array}{r}76.5 \\
76.5 \\
111.0\end{array}$ & & $\begin{array}{l}439.5 \\
440.8 \\
426.0\end{array}$ & $\begin{array}{l}1,838.0 \\
1,843.4 \\
1,782.8\end{array}$ & & $\begin{array}{l}223 . \\
223 . \\
36 .\end{array}$ \\
\hline $\begin{array}{l}\mathrm{C}_{4} \mathrm{H}_{9} \mathrm{Cl}_{-} \\
\mathrm{C}_{6} \mathrm{H}_{4} \mathrm{Cl}_{2} \\
\mathrm{C}_{6} \mathrm{Cl}_{6} \\
\mathrm{C}_{7} \mathrm{H}_{7} \mathrm{Cl} \\
\mathrm{C}_{10} \mathrm{H}_{16} \mathrm{H} \mathrm{Cl}\end{array}$ & $\begin{array}{l}\text { Isobutyl chloride } \\
\text { o-Dichlorobenzene (s) } \\
\text { Hexacllorobenzene (s) } \\
\text { Benzyl chloride.-. } \\
\text { Camphene hydrochloride.-- }\end{array}$ & $\begin{array}{r}92.5 \\
147.0 \\
285.0 \\
126.5 \\
172.5\end{array}$ & & $\begin{array}{r}635.5 \\
671.8 \\
509.0 \\
886.4 \\
1,469.8\end{array}$ & $\begin{array}{l}2,657.7 \\
2,811.5 \\
2,130.2 \\
3,706.9 \\
6,146.7\end{array}$ & & $\begin{array}{l}223 . \\
17 . \\
17 . \\
179 . \\
32 .\end{array}$ \\
\hline $\begin{array}{l}\mathrm{C}_{10} \mathrm{H}_{10} .2 \mathrm{HCl} \\
\mathrm{C}_{10} \mathrm{H}_{16} \mathrm{HCl}_{-.}\end{array}$ & $\begin{array}{l}\text { Terpilene dihydrochloride } \\
\text { Terebenthene hydrochloride }\end{array}$ & $\begin{array}{l}209.0 \\
172.5\end{array}$ & & $\begin{array}{l}1,467.7 \\
1,469.2\end{array}$ & $\begin{array}{l}6,137.9 \\
6,144.2\end{array}$ & & $\begin{array}{l}32 . \\
32 .\end{array}$ \\
\hline $\begin{array}{l}\mathrm{C}_{13} \mathrm{H}_{11} \mathrm{Cl} \\
\mathrm{Cl}_{19} \mathrm{H}_{15} \mathrm{Cl}_{-}\end{array}$ & $\begin{array}{l}\text { Diphenylchloromethane (s) } \\
\text { Triphenylchloromethane (s). }\end{array}$ & $\begin{array}{l}202.5 \\
278.0\end{array}$ & & $\begin{array}{l}1,617.3 \\
2,348.5\end{array}$ & $\begin{array}{l}6,763.6 \\
9,821.4\end{array}$ & & $\begin{array}{l}179 . \\
179 .\end{array}$ \\
\hline
\end{tabular}

62 These compounds are not arranged in classes, but are given according to the number of carbon atoms they contain. In view of the relativity large error in the determinations, the molecular weights are all rounded off. All the data except Thomsen's determinations, or unless otherwise indicated, refer to the formation of a dilute solution of hydrochloric acid. Thomsen's determinations are calculated to the production of gaseous hydrogen chloride. No vacuum correction was applied to any of the values. It is impossible to calculate the heats of combustion of these compounds with any degree of precision, for the amounts of water used in the respective combustions are not specified by any of the writers (except Thomsen) and, hence, it is impossible to correct the values for the heat of dilution of the hydrogen chloride formed.

$$
63 \mathrm{HCl} \text {-gas. }
$$

64 IICl-gas.

(B) COMPOUNDS OF C, H, O AND CL (ALIPHATIC)

\begin{tabular}{|c|c|}
\hline $\mathrm{C}_{2} \mathrm{H}_{3} \mathrm{OCl}$ & Monochloroacetaldehyde \\
\hline $\mathrm{C}_{2} \mathrm{H}_{3} \mathrm{O}_{2} \mathrm{Cl}$ & Monochloroacetic acid (s) \\
\hline $\mathrm{C}_{2} \mathrm{HO}_{2} \mathrm{Cl}_{3}$ & Trichloroacetic acid (s) \\
\hline $\begin{array}{l}\mathrm{C}_{4} \bar{H}_{7} \mathrm{O}_{2} \mathrm{Cl} \\
\mathrm{C}_{4} \mathrm{H}_{6} \mathrm{O}_{2} \mathrm{Cl}\end{array}$ & $\begin{array}{l}\text { Ethyl monochloroacetate } \\
\text { Ethyl dichloroacetate- }\end{array}$ \\
\hline $\mathrm{C}$ & Ethyl dichloroacetate.--- \\
\hline
\end{tabular}

\begin{tabular}{r|r}
78.5 & --- \\
94.5 & --- \\
163.5 & --- \\
122.5 & --- \\
157.0 & ---
\end{tabular}

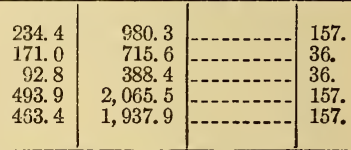

(c) COMPOUNDS OT C, H, O AND CL (AROMATIC)

\begin{tabular}{|c|c|c|c|c|c|c|c|}
\hline $\begin{array}{l}\mathrm{C}_{7} \mathrm{H}_{5} \mathrm{OCl}_{2} \\
\mathrm{C}_{7} \mathrm{H}_{5} \mathrm{O}_{2} \mathrm{Cl}_{-} \\
\mathrm{C}_{7} \mathrm{H}_{5} \mathrm{O}_{2} \mathrm{Cl}_{-\ldots} \\
\mathrm{C}_{7} \mathrm{H}_{4} \mathrm{OCl}_{2} \ldots\end{array}$ & $\begin{array}{l}\text { Benzoyl chloride } \\
\text { o-Chlorobenzoic acid (s) } \\
\text { p-Chlorobenzoic acid (s) } \\
\text { o-Chlorobenzoyl chloride (s) }\end{array}$ & $\begin{array}{l}140.5 \\
156.5 \\
156.5 \\
175.0\end{array}$ & & $\begin{array}{l}782.8 \\
734.5 \\
726.6 \\
741.5\end{array}$ & $\begin{array}{l}3,273.7 \\
3,071.7 \\
3,038.6 \\
3,100.9\end{array}$ & & $\begin{array}{l}157 . \\
157 . \\
157 . \\
157 .\end{array}$ \\
\hline $\begin{array}{l}\mathrm{C}_{7} \mathrm{H}_{5} \mathrm{O}_{2} \mathrm{Cl} \\
\mathrm{C}_{8} \mathrm{H}_{7} \mathrm{OCl}_{2} \\
\mathrm{C}_{8} \mathrm{H}_{4} \mathrm{O}_{2} \mathrm{Cl}_{2} \ldots \\
\mathrm{C}_{9} \mathrm{H}_{9} \mathrm{O}_{2} \mathrm{Cl}_{\ldots}\end{array}$ & $\begin{array}{l}\text { Chlorosalicylaldehyde }{ }^{65} \\
\text { o-Toluyl chloride (s) } \\
\text { Phthalyl chloride (s) } \\
\text { Ethyl o-chlorobenzoate...- }\end{array}$ & $\begin{array}{l}156.5 \\
154.5 \\
203.0 \\
184.5\end{array}$ & & $\begin{array}{r}746.3 \\
944.0 \\
801.8 \\
1,065.8\end{array}$ & $\begin{array}{l}3,121.0 \\
3,947.8 \\
3,353.1 \\
4,457.2\end{array}$ & & $\begin{array}{l}157 . \\
157 . \\
157 . \\
157 .\end{array}$ \\
\hline
\end{tabular}

e5 The position of the chlorine atom in the molecule is not given. 


\title{
VIII. TABLES OF DATA-Continued
}

\section{HALOGEN AND SULPHUR COMPOUNDS-Continued}

\author{
95. CHLORINE COMPOUNDS-Continued
}

(D) CHLOROHYDROQUINOLS

\begin{tabular}{|c|c|c|c|c|c|c|c|}
\hline Formula & Name & $\begin{array}{c}\text { Molec- } \\
\text { ular } \\
\text { weight }\end{array}$ & $\begin{array}{l}\text { Num- } \\
\text { ber } \\
\text { of } \\
\text { elec- } \\
\text { trons }\end{array}$ & $\begin{array}{l}\text { Kg-cal15 } \\
\text { (experi- } \\
\text { mental) }\end{array}$ & $\begin{array}{c}\text { Kilo- } \\
\text { joules } \\
\text { (K. J.) }\end{array}$ & $\begin{array}{l}\text { Kg-cal } 15 \\
\text { (calcu- } \\
\text { lated to } \\
\text { the } \\
\text { liquid } \\
\text { state) }\end{array}$ & Literature \\
\hline $\begin{array}{l}\mathrm{C}_{6} \mathrm{H}_{5} \mathrm{O}_{2} \mathrm{Cl}_{2} \\
\mathrm{C}_{6} \mathrm{H}_{4} \mathrm{O}_{2} \mathrm{Cl}_{2} \\
\mathrm{C}_{6} \mathrm{H}_{3} \mathrm{O}_{2} \mathrm{Cl}_{3} \\
\mathrm{C}_{6} \mathrm{H}_{2} \mathrm{O}_{2} \mathrm{Cl}_{4}\end{array}$ & $\begin{array}{l}\text { Chlorohydroquinol (s) } \\
\text { 2,6-Dichlorohydroquinol (s)- } \\
\text { Trichlorohydroquinol (s) } \\
\text { Tetrachlorohydroquinol (s) }\end{array}$ & $\begin{array}{l}144.5 \\
179.0 \\
213.5 \\
248.0\end{array}$ & & $\begin{array}{l}645.9 \\
614.1 \\
593.0 \\
562.8\end{array}$ & $\begin{array}{l}2,703.1 \\
2,568.2 \\
2,4 S 1.7 \\
2,355.3\end{array}$ & & $\begin{array}{l}225 ; \text { cf. } 222 \text {. } \\
225 \text {; cf. } 222 \text {. } \\
225 \text {; cf. } 222 \text {. } \\
225 \text {; cf. } 223 \text {. }\end{array}$ \\
\hline
\end{tabular}

(E) CHLOROQUINONES

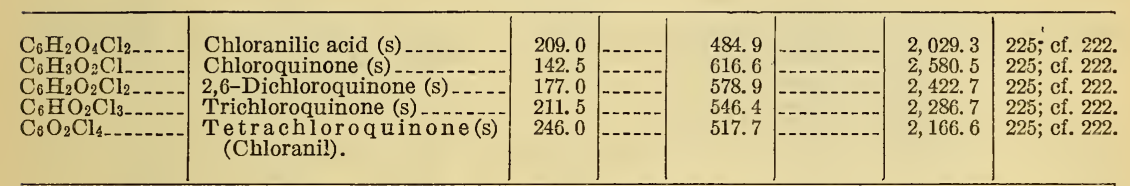

(F) CHLORINE COMPOUNDS OF C, H, O, N

\begin{tabular}{|c|c|c|c|c|c|c|c|}
\hline $\begin{array}{l}\mathrm{C}_{2} \mathrm{H}_{4} \mathrm{ONCl}_{2} \\
\mathrm{C}_{2} \mathrm{H}_{2} \mathrm{ONCl}_{3}\end{array}$ & $\begin{array}{l}\text { Chloroacetamide }(\mathrm{s}) \\
\text { Trichloroacetamide }(\mathrm{s})\end{array}$ & $\begin{array}{r}93.5 \\
162.5\end{array}$ & & $\begin{array}{l}242.5 \\
165.2\end{array}$ & $\begin{array}{r}1,014.1 \\
590.9\end{array}$ & & $\begin{array}{l}157 . \\
157 .\end{array}$ \\
\hline
\end{tabular}

96. $\Lambda$ LKYL BROMIDES 65

\begin{tabular}{|c|c|c|c|c|c|c|c|}
\hline $\mathrm{CH}_{3} \mathrm{Br}$ & Methyl bromide (v) & 95 & & $\left\{\begin{array}{l}184.0 \\
180.4\end{array}\right.$ & 769.5 & & 223. \\
\hline $\mathrm{C}_{2} \mathrm{H}_{5} \mathrm{Br}-$ & Ethyl bromide $(\nabla)$-. & 109.0 & & $\begin{array}{l}340.5 \\
329.5\end{array}$ & $1,423.9$ & $-\cdots$ & 223. \\
\hline $\mathrm{C}_{3} \mathrm{H}_{7} \mathrm{Br}_{\ldots} \ldots$ & Propyl bromide (v) ... & 123.0 & & 497.3 & $2,079.7$ & 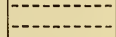 & 233. \\
\hline
\end{tabular}

${ }^{66}$ See footnote 62 , p. 418 , under chlorinated hydrocarbons. The final product of combustion in the case of the bromine derivatives is bromine vapor.

\section{IODINE COMPOUNDS 67}

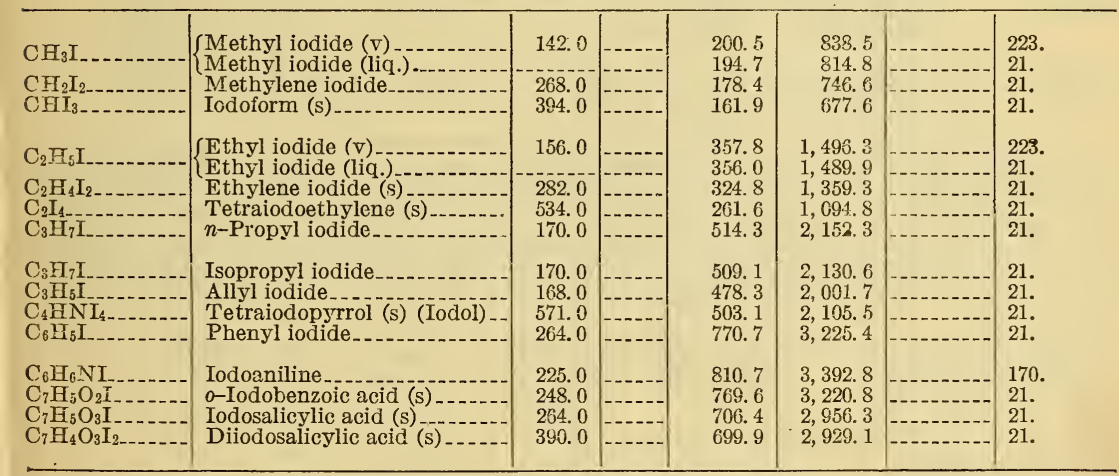

${ }_{67} \mathrm{It}$ is claimed that all the iodo compounds give upon combustion iodine and only inappreciable amounts of $\mathrm{HI}$ or $\mathrm{HIO}_{3}$. Under the conditions of the experiments solid iodine is always the end product. Individual determinations do not agree better than 0.5 to 1.0 per cent. 


\section{TABLES OF DATA-Continued}

\section{HALOGEN AND SULPHUR COMPOUNDS-Continued}

98. SULPHUR COMPOUNDS 68

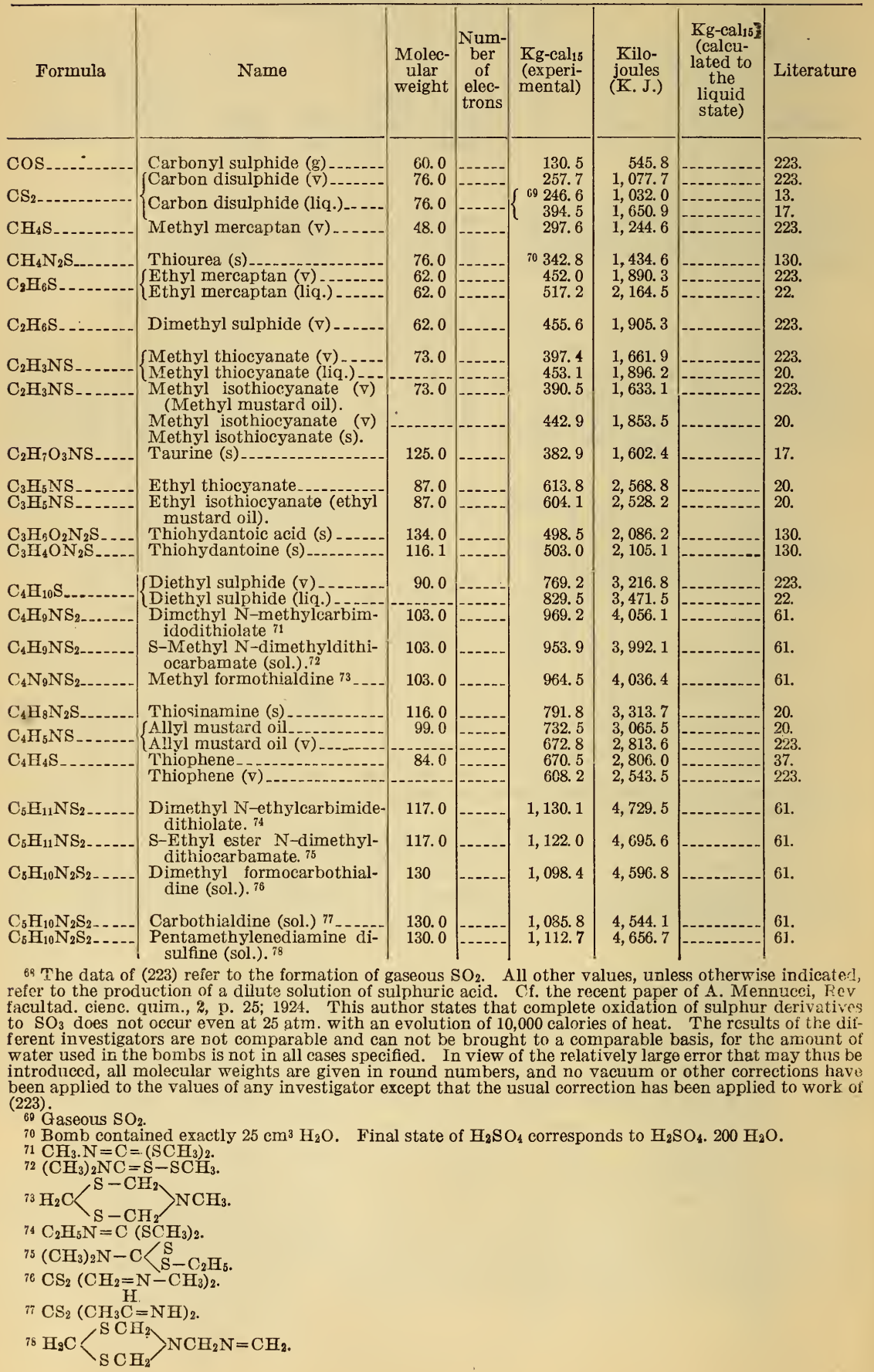




\section{TABLES OF DATA-Continued}

\section{HALOGEN AND SULPHUR COMPOUNDS-Continued}

98. SULPHUR COMPOUNDS-Continued

\begin{tabular}{|c|c|c|c|c|c|c|c|}
\hline Formula & Name & $\begin{array}{c}\text { Molec- } \\
\text { ular } \\
\text { weight }\end{array}$ & $\begin{array}{l}\text { Num- } \\
\text { ber } \\
\text { of } \\
\text { elec- } \\
\text { trons }\end{array}$ & $\begin{array}{l}\text { Kg-cal }{ }_{15} \\
\text { (experi- } \\
\text { mental) }\end{array}$ & $\begin{array}{c}\text { Kilo- } \\
\text { joules } \\
\text { (K. J.) }\end{array}$ & $\begin{array}{l}\text { Kg-cal } 15 \\
\text { (calcu- } \\
\text { lated to } \\
\text { the } \\
\text { liquid } \\
\text { state) }\end{array}$ & Literature \\
\hline $\mathrm{C}_{5} \mathrm{H}_{8} \mathrm{O}_{2} \mathrm{~S}_{-.}$ & Tetrahydro $-\alpha-$ thiophene- & 130.2 & & 754.8 & $3,156.6$ & & 185. \\
\hline $\mathrm{C}_{5} \mathrm{H}_{4} \mathrm{O}_{2} \mathrm{~S}$ & $\alpha$-Thiophenecarboxylic acid & 128.0 & & 646.2 & $2,702.4$ & & 185. \\
\hline $\begin{array}{l}\mathrm{C}_{6} \mathrm{H}_{13} \mathrm{NS}_{2} \ldots \ldots \\
\mathrm{C}_{6} \mathrm{H}_{13} \mathrm{NS}_{2} \ldots \ldots\end{array}$ & Thialdine (sol) ${ }^{79}-$ & $\begin{array}{l}131.0 \\
131.0\end{array}$ & & $\begin{array}{l}1,263.6 \\
1,289.4\end{array}$ & $\begin{array}{l}5,288.2 \\
5,396.1\end{array}$ & & $\begin{array}{l}61 . \\
61 .\end{array}$ \\
\hline $\mathrm{C}_{6} \mathrm{H}_{13} \mathrm{NS}_{2-}$ & $\begin{array}{l}\text { S.Methyl N-diethyldithiocar- } \\
\text { bamate. } 81\end{array}$ & 131.0 & & $1,271.7$ & $5,322.0$ & & 61. \\
\hline $\begin{array}{l}\mathrm{C}_{6} \mathrm{H}_{12} \mathrm{O}_{4} \mathrm{~N}_{2} \mathrm{~S}_{2 \ldots} \ldots \\
\mathrm{C}_{7} \mathrm{H}_{5} \mathrm{NS}_{2}\end{array}$ & Cystine (s) & 240.25 & & 82993.9 & $4,156.5$ & & 19. \\
\hline $\mathrm{C}_{2} \mathrm{H}_{11} \mathrm{NS}_{2-}$ & Dimethyl N-phenylcarbim- & $\begin{array}{l}130.0 \\
165.0\end{array}$ & & $\begin{array}{l}1,024.3 \\
1,544.5\end{array}$ & $6,463.7$ & & 61. \\
\hline $\mathrm{C}_{18} \mathrm{H}_{14} \mathrm{O}_{2} \mathrm{~N}_{2} \mathrm{~S}$ & $\begin{array}{l}\text { Benzonaphthoquinone thia- } \\
\text { zine (s). }\end{array}$ & 322.20 & & $2,278.1$ & $9,533.8$ & & 178. \\
\hline
\end{tabular}

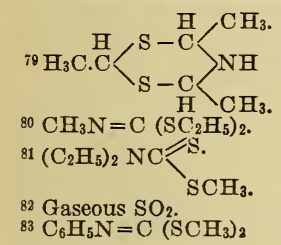

\section{BIBLIOGRAPHY}

1. d'Aladern, Compt. rend., 116, p. 1457; 1893.

2. Andrews, Phil. Mag. (3), 32, p. 321; 1848.

3. Atwater and Snell, J. Amer. Chem. Soc., 25, p. 608; 1903.

4. Auwers and Kolligs, Ber. Chem. Ges., 55, p. 3872; 1922.

5. Auwers and Roth, Lieb. Ann., 3\%3, p. 239; 1910.

6. Auwers, Roth, and Eisenlohr, Lieb. Ann., 373, p. 267; 1910.

7. Auwers, Roth, and Eisenlohr, Lieb. Ann., 385, p. 102; 1911.

8. Barker, J. Phys. Chem., 29, p. 1345; 1925.

9. Berner, Arch. Math. og Naturw. (Oslo), 34, Nr. 6; 1926.

10. Berner, Tidskrift Kemi, 16, pp. 97, 118; 1919.

11. Berner, J. Chem. Soc., 127, p. 2747; 1925.

12. Berthelot, Ann. chim. phys. (5), 23, p. 188; 1881.

13. Berthelot, Ann. chim. phys. (5), 23, p. 209; 1881.

14. Berthelot, Ann. chim. phys. (5), 23, p. 214; 1881.

15. Berthelot, Ann. chim. phys. (5), 23, pp. 243, 252; 1881.

16. Berthelot, Ann. chim. phys. (5), 2\%, p. 374; 1882.

17. Berthelot, Ann. chim. phys. (6), 28, p. 126; 1893.

18. Berthelot, Thermochimie, 11, 1897.

19. Berthelot, Ann. chim. phys. (7), 20, pp. 145, 163, 189; 1900.

20. Berthelot, Ann. chim. phys. (7), 20, p. 197; 1900.

21. Berthelot, Ann. chim. phys. (7), 21, p. 296; 1900.

22. Berthelot, Ann. chim. phys. (7), F2, pp. 322, 327; 1001.

23. Berthelot and André, Ann. chim. phys. (6), 29, p. 5; 1891.

24. Berthelot and André, Ann. chim. phys. (7), 1\%, p. 433; 1899. 
25. Berthelot and Delépine, Compt. rend., 129, p. 920; 1899.

26. Berthelot and Delépine, Ann. chim. phys. (7), 21, p. 289; 1900.

27. Berthelot and Fogh, Ann. chim. phys. (6), 22, p. 18; 1891.

28. Berthelot and Gaudechon, Compt. rend., 140, p. $753 ; 1905$.

29. Berthelot and Jungfleisch, Ann. chim. phys. (5), 6, p. 147; 1875.

30. Berthelot and Louguinine, Ann. chim. phys. (6), 13, p. 321; 1888.

31. Berthelot and Matignon, Ann. chim. phys. (6), 21, p. 409; 1890.

32. Berthelot and Matignon, Ann. chim. phys. (6), 23, p. 538; 1891.

33. Berthelot and Matignon, Compt. rend., 113, p. 246; 1891.

34. Berthelot and Matignon, Compt. rend., 114, p. 1145; 1892.

35. Berthelot and Matignon, Ann. chim. phys. (6), 28, p. 139; 1893.

36. Berthelot and Matignon, Ann. chim. phys. (6), 28, p. 565; 1893.

37. Berthelot and Matignon, Ann. chim. phys. (6), 30, p. 547; 1893.

38. Berthelot and Ogier, Ann. chim. phys. (5), 23, p. 197; 1881.

39. Berthelot and Ogier, Ann. chim. phys. (5), 23, p. 199; 1881.

40. Berthelot and Ogier, Ann. chim. phys. (5), 23, pp. 201, 225; 1881.

41. Berthelot and Petit, Ann. chim. phys. (6), 18, p. 107: 1889.

42. Berthelot and Petit, Ann. chim. phys. (6), 20, pp. 5, 13; 1890.

43. Berthelot and Recoura, Ann. chim. phys. (6), 13, pp. 298, 304, 340; 1888.

44. Berthelot and Rivals, Ann. chim. phys. (7), \%, pp. 29, 47; 1896.

45. Berthelot and Vieille, Ann. chim. phys. (5), 2\%, p. 194; 1882.

46. Berthelot and Vieille, Compt. rend., 102, p. 1284; 1886.

47. Berthelot and Vieille, Ann. chim. phys. (6), 10, p. 433; 1887.

48. Berthelot and Vieille, Ann. chim phys. (6), 10, p. 455; 1887.

49. Blaschko, Biochem. Z., 158, p. 428; 1925.

50. Brühl, Ber., 32, p. 1222; 1899.

51. Bruylants and Christiaen, Bull. soc. chim. Belg., 34, p. 144; 1925.

52. Bureau of Standards, Cir. 11, 3d. ed., 1917.

53. Constam and White, Amer. Chem. J., 29, p. 1; 1903.

54. Coops and Verkade, Rec. trav. chim., 44, p. 983; 1925.

55. Delépine, Compt. rend., 123, p. $650 ; 1896$.

56. Delépine, Compt. rend., 124, p. 1525; 1897.

57. Delépine, Compt. rend., 126ீ, p. 964; 1898.

58. Delépine, Compt. rend., 131, p. $684 ; 1900$.

59. Delépine, Compt. rend., 131, p. 745; 1900.

60. Delépine, Compt. rend., 132, p. 777; 1901.

61. Delépine, Compt. rend., 136, p. 451; 1903.

62. Delépine and Rivals, Compt. rend., 129, p. 520; 1899.

63. Dickinson, B. of S. Bull., 11, p. 189; 1915.

64. Emery and Benedict, Am. J. of Physiol., 28, p. 301; 1911.

65. Favre and Silbermann, Ann. chim. phys. (3), 34, p. 357; 1852.

66. Fischer and Wrede, Sitzber. kgl. preuss, Akad. Wiss., 1904, p. 687.

67. Fischer and Wrede, ZS. phys. Ch., 69, p. 218; 1909.

68. Fogh, Compt. rend., 114, p. 920; 1892.

69. de Forcrand, Ann. chim. phys. (6), 3, p. 187; 1884.

70. Fries, Chem. Zentrbl. (1), p. 1510; 1907. U. S. Dept. Agri. Bur. An. Ind. Bull., 34; 1907.

71. Fries, U. S. Dept. Agri. Bur. An. Ind. Bull. 124; 1910.

72. Garner and Abernethy, Proc. Roy. Soc. London (A), 99, p. 213; 1921.

73. Genvresse, Bull. soc. chim. (3), 9, p. 219; 1893.

74. Gibson, Storrs School Agri. Exper. Sta. Bull. \&, p. 182; 1890.

75. Ginsberg, Diss. Braunschweig; 1923; quoted in Landolt-Börnstein Tabellen; 1923.

76. Gottlieb, J. prakt. Ch. (2), 28, p. 385; 1883. 
77. Guillemard, Ann. chim. phys. (8), 14, p. 311; 1908.

78. Guinchant, Compt. rend., 121, p. 354; 1895; 182, p. 943; 1896; all data collected and evaluated in Ann. de Chim., 10, p. 59; 1918.

79. Hartmann, Proefschr. Delft; 1925.

80. Henning, Z. phys. Ch., 9\%, p. 467; 1921.

81. Hibbert and Fuller, J. Am. Chem. Soc., 35, p. 978; 1913.

82. Hückel, Lieb. Ann., 451, p. 117; 1926.

83. Hückel, Lieb. Ann., 451, p. 160; 1927; Det. by Roth.

84. Jaeger and von Steinwehr, Zeit. phys. chem. 114, p. 59; 1924.

85. Jaeger and von Steinwehr, Zeit. phys. chem., 119, p. 214; 1926.

86. Jahn, Wied. Ann., 3\%, p. 408; 1889.

87. Karrer and Fioroni, Helv. Chim. Acta, 6, p. 396; 1923.

88. Karrer and Fioroni, Ber., 55:

89. Keffler and Guthrie, J. Physic. Chem., 31, p. 68; 1927.

90. Kitran, Farmaceutski vjesnik, 14, pp. 583, 617, 668, 702, 748, 777; 1924.

91. Konovalov, J. Chim. Phys., æ33, 359; 1926.

92. Krummacher, ZS. Biol., 46, p. 310; 1904.

93. Landrieu, Compt. rend., 1140, p. 867; 1905.

94. Landrieu, Compt. rend., 14\%, p. 580; 1906.

95. Landrieu and Blatt, Bull. Soc. Chim., 35, p. 1424; 1924.

96. Lemoult, Compt. rend., 126, p. 343; 1898.

97. Lemoult, Compt. rend., 143, p. 772; 1906.

98. Lemoult, Compt. rend., $\mathbf{1} 3 \mathbf{3}$, p. 902; 1906.

99. Lemoult, Ann. chim. phys. (8), 10, p. 395; 1907.

100. Lemoult, Ann. chim. phys. (8), 13, p. 562; 1908.

101. Lemoult, Ann. chim. phys. (8), 直, pp. 184, 289; 1908.

102. Lemoult, Compt. rend., 148, p. 1602; 1909.

103. Lemoult, 7th Int. Cong. Appi. Chem.; 110, p. 75; 1909.

101. Lemoult, Compt. rend., 15\%, p. 1402; 1911.

105. Leroux, Compt. rend., 151, p. 384; 1910.

106. Leroy, Ann. chim. phys. (7), 21, p. 87; 1900.

107. Leroy, Compt. rend., 130, p. 508; 1900.

108. Liebermann, Ber. chem. Ges., 46, p. 267; 1913; (Det. carried out by Stohmann).

109. Louguinine, Ann. chim. phys. (5), 20, p. 558; 1880.

110. Louguinine, Ann. chim. phys. (5), 21, p. 139; 1880.

111. Louguinine, Compt. rend., 91, p. 297; 1880.

112. Louguinine, Compt. rend., 91, p. 329; 1880.

113. Louguinine, Ann. chim. phys. (5), 23, p. 384; 1881.

114. Louguinine, Compt. rend., 93, p. $274 ; 1881$.

115. Louguinine, Ann. chim. phys. (5), 25, p. 140; 1882.

116. Louguinine, Compt. rend., 98 , p. $94 ; 1884$.

117. Louguinine, Compt. rend., 100, p. 63; 1885.

118. Louguinine, Compt. rend., 101, pp. 1061, 1154; 1885.

119. Louguinine, Ann. chim. phys. (6), 8, p. 128; 1886.

120. Louguinine, Ann. chim. phys. (6), 11, p. 220; 1887.

121. Louguinine, Compt. rend., 106, p. 1289; 1888.

122. Louguinine, Compt. rend., 106, p. 1472; 1888.

123. Louguinine, Compt. rend., 10\%, p. 597; 1888.

124. Louguinine, Compt. rend., 10\%, p. 624; 1888.

125. Louguinine, Ann. chim. phys. (6), 18, p. 378; 1889.

126. Louguinine, Compt. rend., 108, p. 620; 1889.

127. Louguinine, Ann. chim. phys. (6), 23̊, p. 179; 1891.

128. Malbot, Ann. chim. phys. (6), 18, p. 404; 1889.

129. Massol, Ann. chim. phys. (7), 1, p. 145; 1894.

$3687^{\circ}-29-5$ 
130. Matignon, Ann. chim. phys. (6), 28, pp. 70, 289, 498; 1893 . These articles contain also the data reported by Matignon in Compt. rend., 110, p. 1267,$1890 ; 113$, p. 198; 1891.

130.5 Matignon, Compt. rend., 113, p. 198; 1891.

131. Matignon and Deligny, Compt. rend., 121, p. 422; 1895.

132. Matignon and Deligny, Compt. rend., 125, p. 1103; 1897.

133. Meier and Meyerhof, Biochem. Z., 150, p. 233; 1924.

134. Meyerhof, Biochem. Z., 129, p. 594; 1922.

135. Meyerhof, Pfiüg. Arch., 195, pp. 22, 24; 1922.

136. Mixter, Sill. J. (4), 12, p. 347; 1901.

137. Mixter, Sill. J. (4), 22, p. 13; 1906.

138. Moureu and André, Ann. chim. (9), 1, p. 113; 1914.

139. Moureu and Bongrand, Compt. rend., 1\%0, p. 1025; 1920.

140. Moureu and Boutaric, J. chim. phys., 18, p. 348; 1920.

141. Moureu, Boutaric, and Dufraisse, J. chim. phys., 18, p. 333; 1921.

142. Muller, Ann. chim. phys. (8), 20, p. 116; 1910.

143. Muller, Bull. soc. chim. (2), 44, p. 608; 1885.

144. Ossipov, Z. physik. Ch., 2, p. $646 ; 1888$.

145. Ossipov, Ann. chim. phys. (6), 20, p. 371; 1890.

146. Ossipov, J. de la Soc. Chim. russe, 22, p. 317; 1890.

147. Petit, Ann. chim. phys. (6), 18, p. 145; 1889.

148. Pushin, Z. physik. Chem., 124, p. 16; 1926.

149. Rabe and Pollock, Ber. Chem. Ges., 45, p. 2924; 1912.

150. Van Rechenberg, J. prakt. Chem. (2), జ2, p. 11; 1880.

151. Reyer, Diss., Braunschweig; 1923; quoted in Landolt-Börnstein Tabellen; 1923.

152. Richards and Barry, J. Am. Chem. Soc., 3\%, p. 993; 1915.

153. Richards and Davis, J. Am. Chem. Soc., 39, p. 341; 1917; 42, p. 1599, 1920.

154. Richards and Jesse, J. Am. Chem. Soc., 32, p. 268; 1910.

155. Riiber and Schetelig, ZS. ph. Ch., 48, p. 345; 1904.

156. Rinkenbach, Ind. Eng. Chem., 19, p. 474; 1927.

157. Rivals, Ann. chim. phys. (7), 12, p. 501; 1897.

158. Rosenhain, J. Soc. chem. Ind., 25, p. 239; 1906.

159. Roth, Lieb. Ann., 3\%3, p. 249; 1910.

160. Roth, Rec. trav. chim., 45, p. 248; 1926 . (Value by Lemoult corr. by Roth.)

161. Roth, ZS. Elektrochem., 16, p. 654; 1910.

162. Roth, ZS. Elektrochem., 1\%, p. 789; 1911.

163. Roth, Unpub. work. Authority for these values in Landolt-Börnstein Tabellen; 1923.

164. Roth, Landolt-Börnstein Tabellen; 1927.

165. Roth and v. Auwers, Lieb. Ann., 40\%, p. 145; 1915.

166. Roth and v. Auwers, Landolt-Börnstein Tabellen; 1927.

167. Roth and Ellinger, Landolt-Börnstein Tabellen; 1923.

167.5. Roth and Ellinger, Landolt-Börnstein Tabeilen; 1912.

168. Roth and Ellinger, Landolt-Börnstein Tabellen; 1927.

169. Roth and Lassé, Lieb. Ann., 441, p. 48; 1925.

170. Roth and Macheleldt, Diss: Braunschweig; 1921; quoted in LandoltBörnstein Tabellen; 1923.

171. Roth and Moosbrugger, Landolt-Börnstein Tabellen; 1923.

172. Roth and Müller, Landolt-Börnstein Tabellen; 1927.

173. Roth and Müller, Ber., 60, p. 643; 1927.

174. Roth and Murawski, Landolt-Börnstein Tabellen; 1923.

175. Roth and Östling, Ber. Chem. Ges., 46, p. 309; 1913.

176. Roth and Peters, Landolt-Börnstein Tabellen; 1923.

177. Roth and Stoermer, Ber. chem. Ges., 46, p. 260; 1913.

178. Roth and Wolff, Landolt-Börnstein Tabellen; 1927. 
179. Schmidlin, Ann. chim. phys. (8), \%, p. 195; 1906.

180. Schreiner, Z. physik. Chem., 11\%, p. $57 ; 1925$.

181. Stohmann, Journ. prakt. Ch. (2), 31, p. 273; 1885.

182. Stohmann, ZS. ph. Ch., 6, p. 334; 1890.

183. Stohmann, ZS. ph. Ch., 10, p. 410; 1892.

184. Stohmann and Haussmann, J. prakt. Ch. (2), 55, p. 263; 1897. For details consult Berichte über die Verhandl. der kgl. Sächs. Ges. der Wissensch. zu Leipzig, 49, p. 1; 1897.

185. Stohmann and Kleber, J. prakt. Ch. (2), 43, p. 1; 1891.

186. Stohmann and Kleber, J. prakt. Ch. (2), 43, p. 538; 1891.

187. Stohmann and Kleber, J. prakt. Ch. (2), 45, p. 475; 1892.

188. Stohmann, Kleber, and Langbein, J. prakt. Ch. (2), 40, p. 77; 1889.

189. Stohmann, Kleber, and Langbein, J. prakt. Ch. (2), 40, p. $128 ; 1889$.

190. Stohmann, Kleber, and Langbein, J. prakt. Ch. (2), 40, p. 202; 1889.

191. Stohmann, Kleber, and Langbein, J. prakt. Chem. (2), 40, p. 341; 1889.

192. Stohmann, Kleber, Langbein, and Offenhauer, J. prakt. Ch. (2), 49, p. 99; 1894. For details of the work consult Berichte über die Verhandl. der kgl. Sächs. Ges. der Wissensch. zu Leipzig, 45, p. 604; 1893.

193. Stohmann and Langbein, J. prakt. Ch., 42, p. 361; 1890.

194. Stohmann and Langbein, J. prakt. Ch., 44, p. 336; 1891.

195. Stohmann and Langbein, J. prakt. Ch. (2), 45, p. 305; 1892.

196. Stohmann and Langbein, J. prakt. Ch. (2), 46, p. 530; 1893. For details consult Berichte über die Verhandl. der kgl. Sächs. Ges. der Wissensch. zu Leipzig., 49, p. 1; 1897.

197. Stohmann and Langbein, J. prakt. Ch. (2), 48, p. 447; 1893.

198. Stohmann and Langbein, J. prakt. Ch. (2), 49, p. 483; 1894. For details consult Berichte über die Verhandl. der kgl. Sächs. Ges. der Wissensch. zu Leipzig, 49, p. 1; 1897.

199. Stohmann and Langbein, J. prakt. Ch. (2), 50, p. 388; 1894 . For details consult Berichte über die Verhandl. der kgl. Sächs. Ges. der Wissensch. zu Leipzig, 49, p. 1; 1897.

200. Stohmann, Rodatz, and Herzberg, J. prakt. Ch. (2), 33, p. 241; 1886.

201. Stohmann, Rodatz, and Herzberg, J. prakt. Ch. (2), 33, p. 464; 1886.

202. Stohmann, Rodatz, and Herzberg, J. prakt. Ch. (2), 34, p. 311; 1886.

203. Stohmann, Rodatz, and Herzberg, J. prakt. Ch. (2), 35, p. 22; 1887.

204. Stohmann, J. prakt. Ch. (2), 35, pp. 40, 136; 1887.

205. Stohmann, Rodatz, and Herzberg, J. prakt. Ch. (2), 36, p. 1; 1887.

206. Stohmann, Rodatz, and Herzberg, J. prakt. Ch. (2), 36, p. 353; 1887.

207. Stohmann and Schmidt, J. prakt. Ch. (2), 50, p. 385; 1894.

208. Stohmann and Schmidt, J. prakt. Ch. (2), 52, p. 59; 1895 . For details consult Berichte über die Verhandl. der kgl. Sächs. Ges. der Wissensch. zu Leipzig, 4\%, p. 1; 1895.

209. Stohmann and Schmidt, J. prakt. Ch. (2), 53, p. 345; 1896.

210. Swarts, Bull Acad. Belg., 14; 1924.

211. Swarts, Rec. Trav. Chim. Pays Bas, 32, p. 59; 1913; J. chim. phys., 17 , p. $3 ; 1919$.

212. Swietoslawski, ZS. ph. ch., 92, p. 49; 1910.

213. Swietoslawski, J. Russ. Phys. Chem. Soc., 46, p. 1302; 1914.

214. Swietoslawski, J. Am. Chem. Soc., 39, p. 2595; 1917.

215. Swietoslaswki, J. Am. Chem. Soc., 42, p. 1092; 1920.

216. Swietoslawski, J. chim. phys., 22, p. 583; 1925.

217. Swietoslawski, Private comm.

218. Swietoslawski and Pakovick, J. Russ. Phys. Chem. Soc., 46, p. 1284; 1914.

219. Swietoslawski and Popov, J. Russ. Phys. Chem. Soc., 46, p. 935; 1914.

220. Swietoslawski, Popov, and Pakovick, J. Russ. Phys. Chem. Soc., 46, p. $1293 ; 1914$.

221. Swietoslawski and Starczewska, Bull. soc. chim. (4), 31, p. 654; 1922. 
222. Swietoslawski and Starczewska, J. chim. phys., 22, p. 399; 1925.

223. Thomsen, Thermoch. Unters. IV: letzte Zusammenstellung; ZS. ph. Ch., 5\%, p. 343; 1905.

224. Tower, Value reported by Atwater and Snell, J. Am. Chem. Soc., 25, p. $608 ; 1903$.

225. Valeur, Ann. chim. phys. (7), 21, p. 470; 1900.

226. Verkade, Amst. Akad. Versl., 35, p. 496; 1926. Values by Verkade, van de Lee and Coops.

227. Verkade, Proefschr. Delft; 1915.

228. Verkade, Chem. Weekbl., 19, p. 389; 1922.

229. Verkade and Coops, Rec. Tr. Chim. P. B., 45, p. 551; 1926.

230. Verkade and Coops, Rec. Trav. Chim. P. B., 193, p. 561; 1924.

230.5. Verkade and Coops, Rec. Trav. Chim, 42, p. 223; 1923.

231. Verkade and Coops, Private comm.

232. Verkade, Coops and Hartmann, Rec. Trav. Chim. P. B., 41, p. 241; 1922.

233. Verkade, Hartmann and Coops, Rec. Tr. Chim. P. B., 45 , p. 585; 1926.

234. Verkade and Hartmann, Priv. comm.

235. Verkade, Hartmann and Coops, Proc. Acad. Sc. Amsterdam, 2\%, p. 859; 1924.

236. Verkade, Verkade and Coops, Landolt-Börnstein Tabellèn; 1927.

237. Verkade, Verkade, Coops, and Maan, Priv. comm.

238. Voisenet, Bull. soc. chim. (4), 1\%, p. 34; 1915.

239. Wartenberg, Muchlinski, and Riedler, Z. Angew. Chem., 3\%, p. 457; 1924.

240. Weigert, ZS. phys. Ch., 63, p. 458; 1908.

241. Wrede, ZS. phys. Ch., \%5, p. 81; 1910.

242. Wrede, Landolt-Börnstein Tabellen; 1927.

243. Zubov, J. Russ. Phys. Chem. Soc. (7), 28, p. 687; 1896.

244. Zubov, J. Russ. Chem. Phys. Soc. (7), 30, p. 926; 1398.

245. Zubov and Swietoslawski, Bull. Soc. Chim., 3\%, p. 271; 1925.

246. Bureau of Standards, Unpublished work.

\section{INDEX OF COMPOUNDS, BY FORMULA}

1. $\mathrm{CH}$ COMPOUNDS

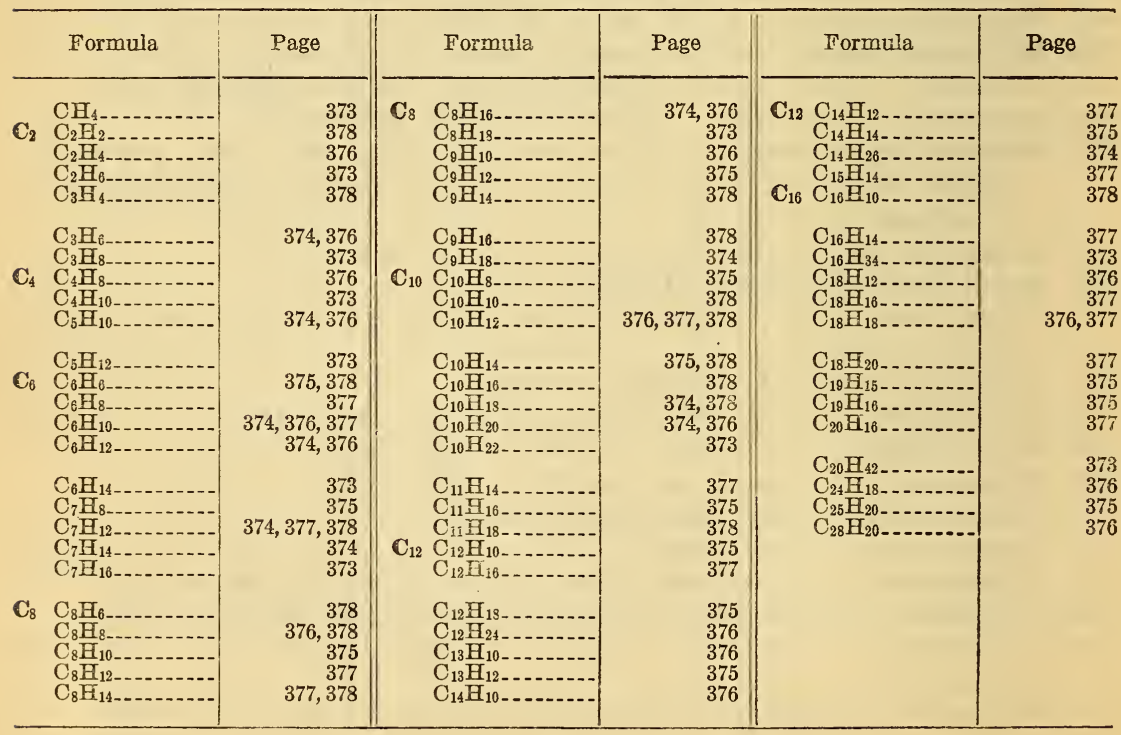




\section{INDEX OF COMPOUNDS, BY FORMULA-Continued}

\section{CHO COMPOUNDS}

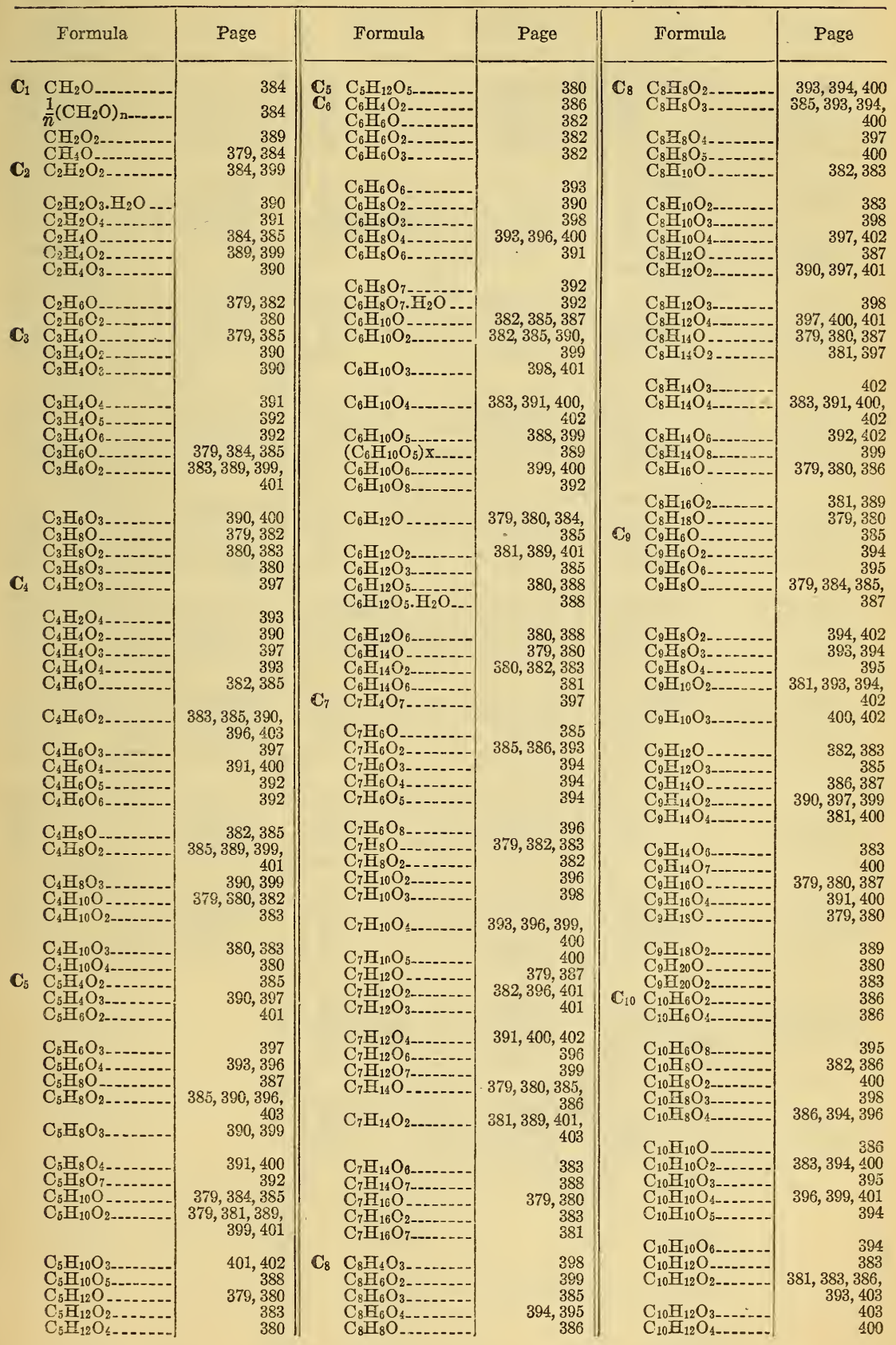




\section{INDEX OF COMPOUNDS, BY FORMULA-Continued}

\section{CHO COMPOUNDS-Continued}

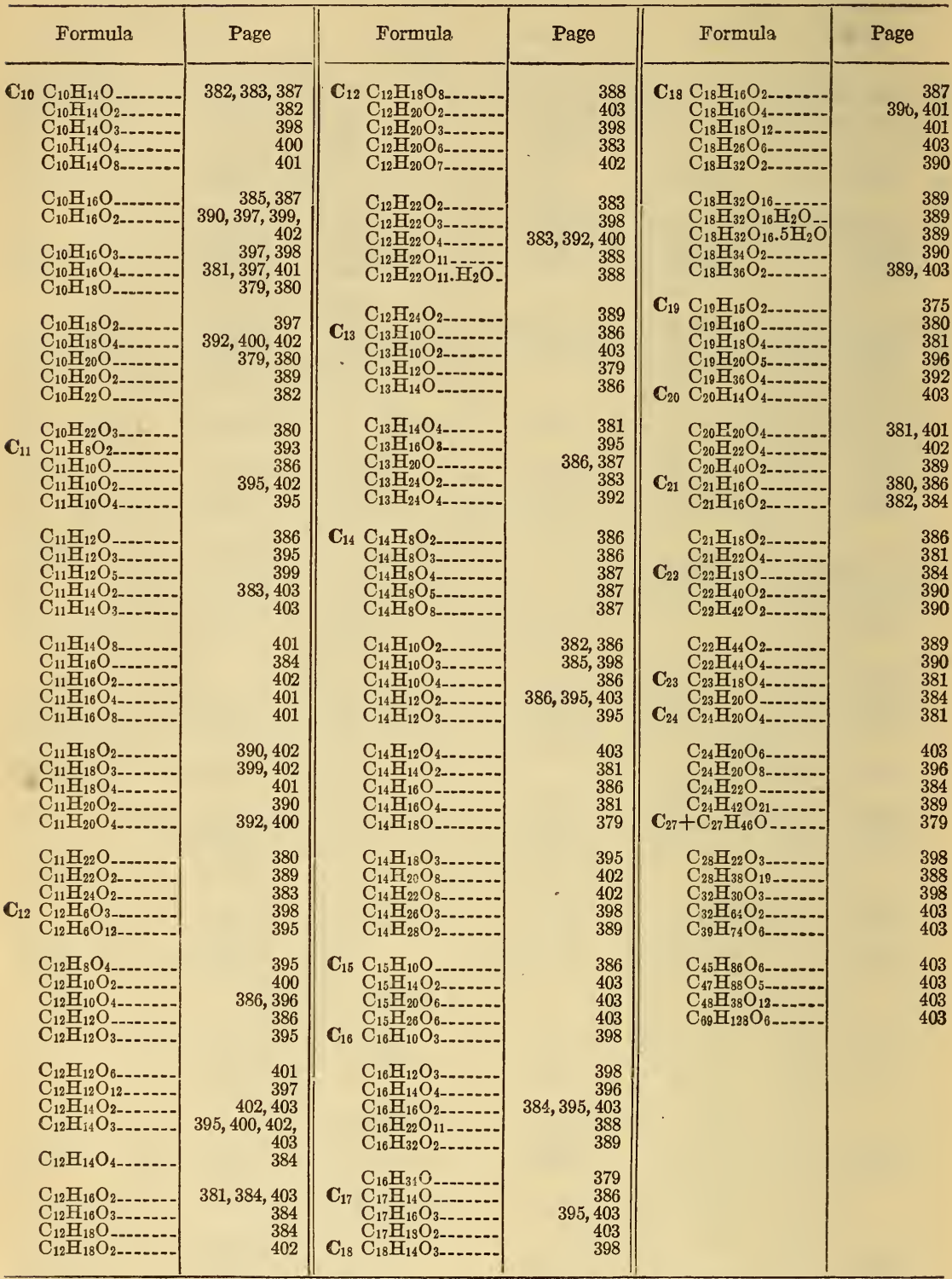




\section{INDEX OF COMPOUNDS, BY FORMULA-Continued}

\section{3. $\mathrm{N}$ COMPOUNDS}

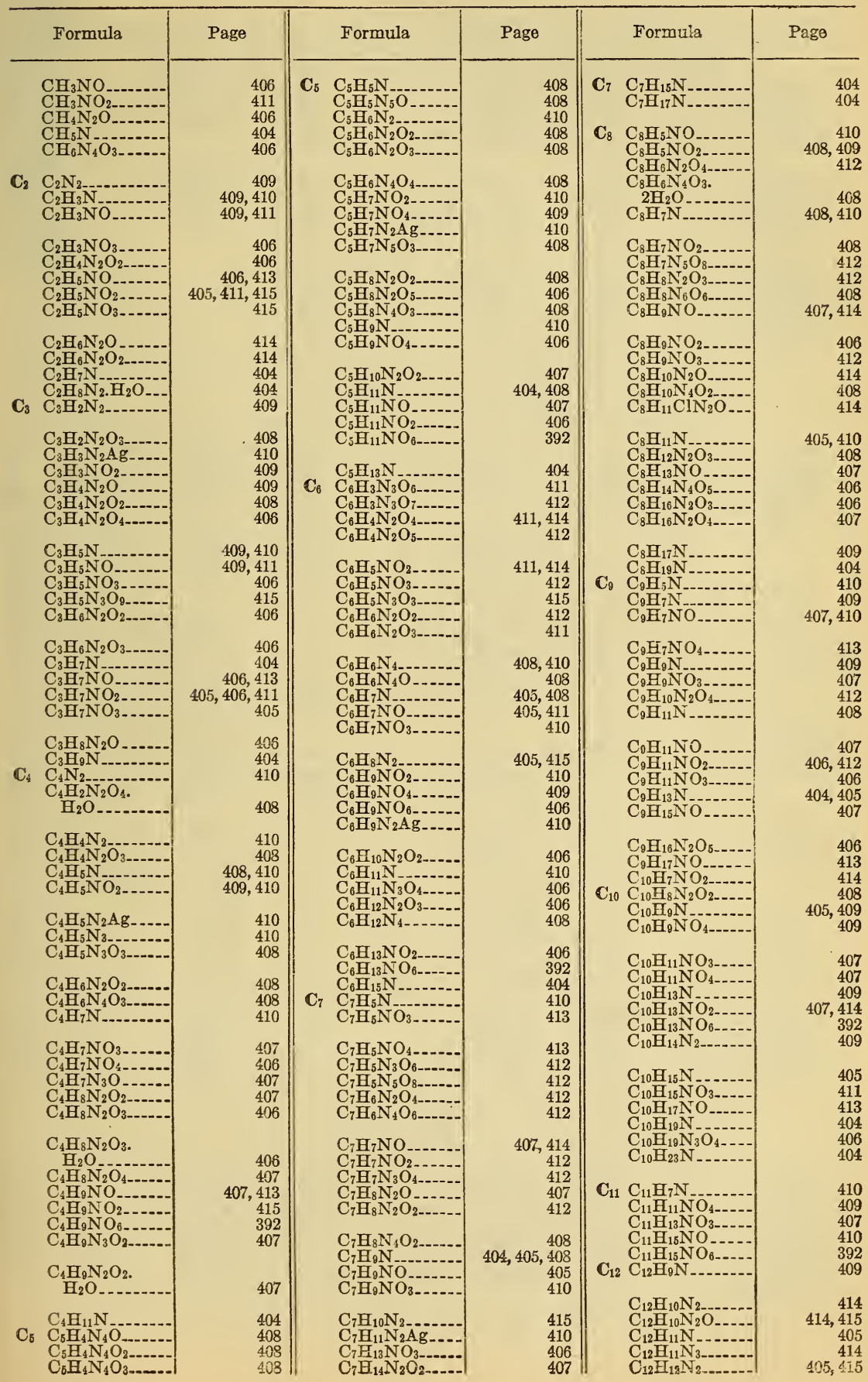




\section{INDEX OF COMPOUNDS, BY FORMULA-Continued}

\section{N COMPOUNDS-Continued}

\begin{tabular}{|c|c|c|c|c|c|}
\hline Formula & Page & Formula & Page & Formula & Page \\
\hline $\begin{aligned} & \mathbf{C}_{12} \mathrm{C}_{12} \mathrm{H}_{12} \mathrm{~N}_{4} \\
& \mathrm{C}_{12} \mathrm{H}_{14} \mathrm{~N}_{4} \mathrm{O}_{6} \ldots \ldots \\
& \mathrm{C}_{12} \mathrm{H}_{14} \mathrm{~N}_{4} \mathrm{O}_{8} \ldots \ldots \\
& \mathrm{C}_{12} \mathrm{H}_{22} \mathrm{~N}_{2} \mathrm{O}_{2} \ldots \ldots \\
& \mathrm{C}_{12} \mathrm{H}_{27} \mathrm{~N}_{\ldots}\end{aligned}$ & $\begin{array}{l}414 \\
408 \\
408 \\
406 \\
404\end{array}$ & $\begin{aligned} & \mathrm{C}_{16} \mathrm{C}_{16} \mathrm{H}_{12} \mathrm{~N}_{2} \mathrm{O}_{4} \\
& \mathrm{C}_{16} \mathrm{H}_{13} \mathrm{~N} \\
& \mathrm{C}_{16} \mathrm{H}_{13} \mathrm{NO}_{3} \\
& \mathrm{C}_{16} \mathrm{H}_{15} \mathrm{NO}_{3} \\
& \mathrm{C}_{16} \mathrm{H}_{15} \mathrm{~N}_{2} \mathrm{O}_{2}\end{aligned}$ & $\begin{array}{l}408 \\
405 \\
407 \\
407 \\
407\end{array}$ & $\begin{array}{r}\mathrm{C}_{20} \mathrm{C}_{20} \mathrm{H}_{21} \mathrm{NO}_{4} \\
\mathrm{C}_{20} \mathrm{H}_{27} \mathrm{~N} \mathrm{O}_{11} \\
\mathrm{C}_{21} \mathrm{~B}_{13} \mathrm{~N} \mathrm{O}_{5} \\
\mathrm{C}_{21} \mathrm{H}_{21} \mathrm{~N}\end{array}$ & $\begin{array}{l}409 \\
409 \\
412 \\
404\end{array}$ \\
\hline $\begin{array}{ll}\mathbf{C}_{13} & \mathrm{C}_{13} \mathrm{H}_{11} \mathrm{NO} \mathrm{O}_{2} \\
& \mathrm{C}_{13} \mathrm{H}_{12} \mathrm{~N}_{2} \mathrm{O}_{-} \\
\mathbf{C}_{14} & \mathrm{C}_{14} \mathrm{H}_{2} \mathrm{NO}_{4} \\
& \mathrm{C}_{14} \mathrm{H}_{14} \mathrm{~N}_{2} \mathrm{O}_{2 \ldots} \\
& \mathrm{C}_{14} \mathrm{H}_{15} \mathrm{~N}_{2}\end{array}$ & $\begin{array}{r}407,414 \\
407 \\
412 \\
414 \\
404\end{array}$ & $\begin{array}{ll} & \mathrm{C}_{16} \mathrm{H}_{18} \mathrm{~N}_{2} \mathrm{O}_{2} \ldots \\
& \mathrm{C}_{16} \mathrm{H}_{18} \mathrm{~N}_{2} \mathrm{O}_{3} \\
& \mathrm{C}_{17} \mathrm{H}_{19} \mathrm{NO}_{3} \mathrm{H}_{2} \mathrm{O} \\
\mathrm{C}_{18} & \mathrm{C}_{18} \overline{\mathrm{H}}_{15} \mathrm{~N} \\
& \mathrm{C}_{18} \mathrm{H}_{21} \mathrm{NO} \mathrm{O}_{3} \mathrm{H}_{2} \mathrm{O}\end{array}$ & $\begin{array}{l}414 \\
415 \\
409 \\
405 \\
409\end{array}$ & $\begin{array}{l}\mathrm{C}_{21} \mathrm{H}_{22} \mathrm{~N}_{2} \mathrm{O}_{2} \ldots \\
\mathrm{C}_{22} \mathrm{H}_{23} \mathrm{~N}_{7}-\ldots \\
\mathrm{C}_{23} \mathrm{H}_{23} \mathrm{~N}_{2} \mathrm{O}_{4-\ldots} \\
\mathrm{C}_{23} \mathrm{H}_{27} \mathrm{NO}_{8} \\
2 \mathrm{H}_{2} \mathrm{O}\end{array}$ & $\begin{array}{l}409 \\
409 \\
409 \\
409\end{array}$ \\
\hline $\begin{aligned} & \mathrm{C}_{14} \mathrm{H}_{16} \mathrm{~N}_{4} \mathrm{O}_{2} \\
\mathrm{C}_{15} \mathrm{H}_{13} \mathrm{~N}_{2} & \\
\mathrm{C}_{16} & \mathrm{C}_{16} \mathrm{H}_{10} \mathrm{~N}_{2} \mathrm{O}_{2} \\
\mathrm{C}_{16} \mathrm{H}_{11} \mathrm{NO}_{2} & \\
\mathrm{C}_{16} \mathrm{H}_{11} \mathrm{NO}_{5} & \end{aligned}$ & $\begin{array}{l}415 \\
404 \\
409 \\
407 \\
412\end{array}$ & $\begin{array}{l}\mathrm{C}_{10} \mathrm{H}_{13} \mathrm{~N}_{3} \mathrm{O}_{3} \ldots \\
\mathrm{C}_{19} \mathrm{H}_{13} \mathrm{~N}_{3} \mathrm{O}_{7} \ldots \\
\mathrm{C}_{19} \mathrm{H}_{19} \mathrm{~N}_{3} \mathrm{O}_{\ldots} \ldots \\
\mathrm{C}_{19} \mathrm{H}_{21} \mathrm{NO}_{3 \ldots} \ldots\end{array}$ & $\begin{array}{l}411 \\
411 \\
405 \\
409\end{array}$ & . & \\
\hline
\end{tabular}

\section{HALOGEI COMPOUNDS}

\begin{tabular}{|c|c|c|c|c|c|c|c|c|}
\hline & $\mathrm{ESS}^{\mathrm{r}}$ & \multirow{5}{*}{$\begin{array}{l}419 \\
419 \\
419\end{array}$} & \multirow{4}{*}{\multicolumn{2}{|c|}{ 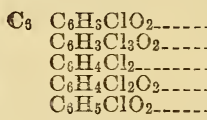 }} & \multirow{4}{*}{$\begin{array}{l}419 \\
419 \\
418 \\
419 \\
419\end{array}$} & \multirow[t]{6}{*}{$\mathbf{C}_{6}$} & \multirow{4}{*}{$\begin{array}{l}\mathrm{C}_{6} \mathrm{H}_{5} \mathrm{FO} \\
\mathrm{C}_{6} \mathrm{~F}_{3} \mathrm{FN} \\
\mathrm{C}_{7} \mathrm{H}_{4} \mathrm{H}_{3} \mathrm{NiO}_{2} \\
\mathrm{C}_{7} \mathrm{H}_{5} \mathrm{~F}_{3} \\
\mathrm{C}_{7} \mathrm{H}_{5} \mathrm{FO}_{2}\end{array}$} & \multirow{4}{*}{$\begin{array}{l}416 \\
417 \\
417 \\
415 \\
416\end{array}$} \\
\hline & $\mathrm{CH}_{3} \mathrm{Bi}_{2}$ & & & & & & & \\
\hline & $\begin{array}{l}\mathrm{C}_{2} \mathrm{H}_{5} \mathrm{Br} \\
\mathrm{C}_{3} \mathrm{H}_{7} \mathrm{Br}\end{array}$ & & & & & & & \\
\hline & $\mathrm{C}_{3} \mathrm{H}_{7} \mathrm{Br}$ & & & & & & & \\
\hline & Cl & & & $\begin{array}{l}\mathrm{C}_{7} \mathrm{H}_{4} \mathrm{Cl}_{2} \mathrm{O}_{7} \\
\mathrm{C}_{7} \mathrm{H}_{5} \mathrm{ClO}\end{array}$ & $\begin{array}{l}418 \\
418\end{array}$ & & $\mathrm{C}_{7} \mathrm{H}_{6} \mathrm{~F}_{2-}$ & $\begin{array}{l}416 \\
415\end{array}$ \\
\hline $\mathbb{C}_{1}$ & $\mathrm{CCl}_{2} \ldots$ & $\begin{array}{l}418 \\
418\end{array}$ & & $\begin{array}{l}\mathrm{C}_{7} \mathrm{H}_{5} \mathrm{ClO}_{2} \\
\mathrm{C}_{7} \mathrm{H}_{7} \mathrm{Cl}_{-}\end{array}$ & $\begin{array}{l}418 \\
418\end{array}$ & & $\begin{array}{l}\mathrm{C}_{7} \mathrm{H}_{7} \mathrm{~F}- \\
\mathrm{C}_{7} \mathrm{H}_{15} \mathrm{~F}-\end{array}$ & $\begin{array}{l}415 \\
415\end{array}$ \\
\hline & $\mathrm{CH}_{2} \mathrm{Cl}_{2}$ & & $C_{8}$ & $\begin{array}{l}\mathrm{C}_{8} \mathrm{H}_{4} \mathrm{Cl}_{2} \mathrm{O}_{2} \\
\mathrm{C}_{8} \mathrm{H}_{7} \mathrm{ClO} \mathrm{Cl}_{-}\end{array}$ & $\begin{array}{l}418 \\
418\end{array}$ & $\mathbb{C}_{8}$ & $\mathrm{C}_{8} \mathrm{H}_{5} \mathrm{~T}_{5} \mathrm{O}$ & \\
\hline $\mathbb{C}_{2}$ & $\begin{array}{l}\mathrm{CH}_{3} \mathrm{Cl}- \\
\mathrm{C}_{2} \mathrm{Cl}_{4-.}\end{array}$ & $\begin{array}{l}418 \\
418\end{array}$ & $\mathbf{C}_{10}$ & $\begin{array}{l}\mathrm{C}_{9} \mathrm{H}_{9} \mathrm{ClO}_{2-} \\
\mathrm{C}_{10} \mathrm{H}_{17} \mathrm{Cl}_{2}\end{array}$ & $\begin{array}{l}418 \\
418\end{array}$ & & $\begin{array}{l}\mathrm{C}_{8} \mathrm{H}_{7} \mathrm{FN}_{2} \mathrm{~N}_{2} \\
\mathrm{C}_{8} \mathrm{H}_{7} \mathrm{~F}_{2} \mathrm{NO}\end{array}$ & $\begin{array}{l}417 \\
417 \\
417\end{array}$ \\
\hline & $\begin{array}{l}\mathrm{C}_{2} \mathrm{Cl}_{3} \\
\mathrm{C}_{2} \mathrm{HCl}_{3} \mathrm{O}_{2}\end{array}$ & $\begin{array}{l}418 \\
418\end{array}$ & & $\begin{array}{l}\mathrm{C}_{10} \mathrm{H}_{18} \mathrm{Cl} \mathrm{Cl}_{2-} \\
\mathrm{C}_{18} \mathrm{H}_{11} \mathrm{Cl} \ldots\end{array}$ & $\begin{array}{l}418 \\
418\end{array}$ & & $\begin{array}{l}\mathrm{C}_{8} \mathrm{H}_{8} \mathrm{FTNO}_{8} \mathrm{FNO}_{3} \\
\mathrm{CHNO}_{3}\end{array}$ & $\begin{array}{l}417 \\
417\end{array}$ \\
\hline & $\mathrm{C}_{2} \mathrm{H}_{2}$ & 419 & & $\mathrm{C}_{19} \mathrm{H}_{15} \mathrm{Cl}$ & 418 & & $\mathrm{C}_{8} \mathrm{H}_{3} \mathrm{FO}-$ & 416 \\
\hline & $\mathrm{C}_{2} \mathrm{H}_{3} \mathrm{Cl}$ & 418 & & $F$ & & & $\mathrm{C}_{9} \mathrm{H}_{7} \mathrm{FO}_{2}^{-}$ & 416 \\
\hline & $\begin{array}{l}\mathrm{C}_{2} \mathrm{H}_{3} \mathrm{ClO}_{-} \\
\mathrm{C}_{3} \mathrm{H}_{3} \mathrm{ClO}_{2}\end{array}$ & $\begin{array}{l}418 \\
418\end{array}$ & $\mathbb{C}_{2}$ & $\mathrm{C}_{2} \mathrm{H}_{2} \mathrm{~F}_{2} \mathrm{O}_{2}$ & 416 & & $\begin{array}{l}\mathrm{C}_{9} \mathrm{H}_{9} \mathrm{~F}_{3} \mathrm{NO} \\
\mathrm{C}_{9} \mathrm{H}_{9} \mathrm{~F} \mathrm{O}_{2}\end{array}$ & $\begin{array}{l}417 \\
416\end{array}$ \\
\hline & $\mathrm{C}_{2} \mathrm{H}_{4} \mathrm{CINO}$ & 419 & & $\begin{array}{l}\mathrm{C}_{2} \mathrm{Hi}_{3} \mathrm{~F}_{2} \mathrm{NO}_{2} \\
\mathrm{H}_{3} \mathrm{FO}_{2}\end{array}$ & $\begin{array}{l}416 \\
416\end{array}$ & & $\mathrm{C}_{9} \mathrm{H}_{11} \mathrm{~F}-$ & 415 \\
\hline & $\mathrm{C}_{2} \mathrm{H}_{4} \mathrm{Cl}_{2}$ & 418 & & $\mathrm{C}_{2} \mathrm{H}_{4} \mathrm{FNO}$ & 416 & & I & \\
\hline $\mathbb{C}_{3}$ & $\mathrm{C}_{3} \mathrm{H}_{4} \mathrm{Cl}_{2}-\cdots$ & 418 & & $\mathrm{C}_{2} \mathrm{H}_{4} \mathrm{H}_{4} \mathrm{~F}_{2} \mathrm{O}$ & 415 & & $\mathrm{CHI}_{3-}$ & \\
\hline & $\begin{array}{l}\mathrm{C}_{3} \mathrm{H}_{5} \mathrm{Cl} \ldots \\
\mathrm{C}_{3} \mathrm{H}_{6} \mathrm{Cl}_{2}\end{array}$ & $\begin{array}{l}418 \\
418\end{array}$ & & $\mathrm{C}_{2} \mathrm{H}_{5} \mathrm{~F}_{2} \mathrm{~N}$ & 417 & & $\mathrm{CH}_{2} \mathrm{I}_{2-}$ & 419 \\
\hline & & & $\mathrm{C}_{4}$ & $\mathrm{C}_{4} \mathrm{H}_{6} \mathrm{~F}_{2} \mathrm{O}_{2} \ldots$ & 416 & & & 419 \\
\hline $\mathbb{C}_{4}$ & $\begin{array}{l}\mathrm{C}_{3} \mathrm{H}_{7} \mathrm{Cl}_{1}--- \\
\mathrm{C}_{4} \mathrm{H}_{6} \mathrm{CH}_{2} \mathrm{O}_{2}\end{array}$ & $\begin{array}{l}418 \\
418\end{array}$ & & $\mathrm{C}_{4} \mathrm{H}_{7} \mathrm{~F}_{4} \mathrm{~N}$ & 417 & & $\mathrm{C}_{2} \mathrm{H}_{4} \mathrm{I}_{2}$ & 419 \\
\hline & $\mathrm{C}_{2} \mathrm{HT}_{7} \mathrm{ClO}_{2}$ & 418 & & 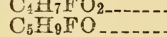 & $\begin{array}{l}416 \\
416\end{array}$ & & $\begin{array}{l}\mathrm{C}_{2} \mathrm{H}_{51} \mathrm{I} \\
\mathrm{C}_{3} \mathrm{E}_{5} \mathrm{I}\end{array}$ & $\begin{array}{l}419 \\
419\end{array}$ \\
\hline $\mathbb{C}_{6}$ & $\mathrm{C}_{6} \mathrm{Ol}_{4} \mathrm{O}_{2}$ & $\begin{array}{l}418 \\
419\end{array}$ & $\mathbb{C}_{6}$ & $\mathrm{C}_{6} \mathrm{H}_{3} \mathrm{FN}_{2} \mathrm{O}_{4}$ & 417 & & $\mathrm{C}_{3} \mathrm{H}_{7} \mathrm{I}$ & 419 \\
\hline & $\mathrm{C}_{6} \mathrm{Cl}_{6-}$ & 418 & & $\mathrm{C}_{6} \mathrm{H}_{4} \mathrm{~T}_{2} \ldots$ & 415 & & 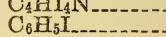 & $\begin{array}{l}419 \\
419\end{array}$ \\
\hline & $\mathrm{C}_{6} \mathrm{HC}_{3} \mathrm{O}_{2}$ & $\begin{array}{l}419 \\
410\end{array}$ & & $\mathrm{C}_{6} \mathrm{H}_{4} \mathrm{FNO}_{2}$ & $\begin{array}{l}417 \\
417\end{array}$ & & $\mathrm{C}_{6} \mathrm{H}_{6} \mathrm{IN}$ & 419 \\
\hline & $\mathrm{C}_{6} \mathrm{II}_{2} \mathrm{Cl}_{2} \mathrm{O}_{4--}$ & $\begin{array}{l}419 \\
419\end{array}$ & & $\mathrm{C}_{6} \mathrm{H}_{5} \mathrm{H}_{5} \mathrm{~F}$ & $\begin{array}{l}416 \\
415\end{array}$ & & $\mathrm{C}_{7} \mathrm{H}_{5} \mathrm{H}_{5} \mathrm{IO}_{3} \ldots$ & $\begin{array}{l}419 \\
419\end{array}$ \\
\hline & $\mathrm{C}_{6} \mathrm{H}_{2} \mathrm{Cl}_{4} \mathrm{O}_{2}$ & 419 & & $\mathrm{C}_{6} \mathrm{H}_{5} \mathrm{FN}_{2} \mathrm{O}_{2}$ & 417 & & $\mathrm{C}_{7} \mathrm{H}_{4} \mathrm{I}_{2} \mathrm{O}_{3-}$ & 419 \\
\hline
\end{tabular}

\section{S COMPOUNDS}

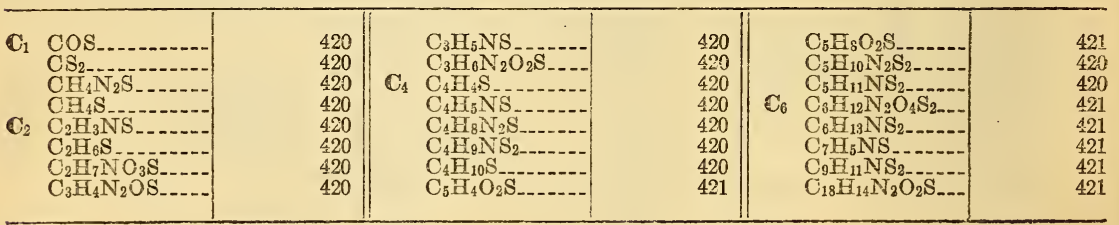

